\title{
O problema de corte não-guilhotinado multiperíodo com sobras aproveitáveis
}

\author{
Oberlan Christo Romão
}

TESE APRESENTADA

AO

Instituto de MATEMÁtica e Estatística

DA

Universidade de São Paulo

PARA

OBTENÇÃO DO TÍTULO

DO

DOUTOR EM CIÊNCIAS

Programa: Ciência da Computação

Orientador: Prof. Dr. Ernesto G. Birgin

Durante o desenvolvimento deste trabalho, o autor recebeu auxílio financeiro do CNPq (146110/2013-7) 
Esta tese é dedicada aos meus pais, Maria do Carmo e Victorino, e a minha amada e eterna namorada Fabíola, que preenche minha vida com alegria e felicidade. 


\section{Agradecimentos}

Em primeiro lugar, agradeço a Deus por todas as alegrias, pela saúde, força e determinação que me concedeu, para que conseguisse chegar até aqui. Agradeço também por sempre colocar pessoas tão especiais ao meu lado.

Aos meus pais e irmãos por sempre acreditarem em mim e pelo apoio em todos os momentos. Sem vocês, nada disso seria possível. Aos meus pais, obrigado por toda a educação que recebi e pela oportunidade que me deram de realizar meus sonhos. Vocês são exemplos de vida para mim.

A minha namorada Fabíola, por sempre acreditar em mim e me incentivar. Pelo companheirismo, paciência, amizade, amor e carinho. Sem ela esse trabalho e minha vida não seriam completos.

Ao meu orientador Ernesto G. Birgin que colaborou de forma fundamental nesse trabalho, indicando sugestões que contribuíram de forma significativa. Obrigado pelos ensinamentos, atenção e dedicação ao longo deste trabalho.

Aos meus amigos, agradeço por todos os momentos divertidos, de alegria e descontração. Obrigado por sempre torcerem pelo meu sucesso. 


\section{Resumo}

ROMÃO, O. C. O problema de corte não-guilhotinado multiperíodo com sobras aproveitáveis. 2017. Tese (Doutorado) - Instituto de Matemática e Estatística, Universidade de São Paulo, São Paulo, 2017.

Neste trabalho, estudamos o problema de corte bidimensional multiperíodo com sobras aproveitáveis, que consiste em cortar objetos grandes visando a produção de um conjunto de itens menores. Supomos um horizonte de planejamento finito com uma quantidade finita de períodos entre os tempos inicial e final. Primeiramente consideramos uma versão determinística em que conhecemos, à priori, os itens solicitados em uma ordem de trabalho e o custo dos objetos a cada período. Algumas das sobras geradas durante o processo de corte dos itens solicitados em um período podem ser utilizadas como objetos no futuro. As sobras que podem ser usadas no futuro são denominadas sobras aproveitáveis. De forma geral, uma sobra é considerada aproveitável se possui dimensões iguais ou superiores as de algum item de uma lista pré-definida para o período. O objetivo é minimizar o custo total dos objetos utilizados para satisfazer a ordem de trabalho dos itens solicitados de todo o horizonte considerado. Havendo soluções com o mesmo custo, desejamos encontrar aquela que, no fim do horizonte de tempo considerado, maximize o valor das sobras aproveitáveis remanescentes. Apresentamos uma modelagem matemática do problema usando uma formulação em dois níveis, que é transformada em um modelo de programação linear inteira mista, devido às características do problema. Considerando a dificuldade em resolver o modelo desenvolvido, apresentamos uma proposta de uma abordagem heurística baseada em Programação Dinâmica Aproximada (PDA) para lidar com o problema proposto. Outras opções baseadas em estratégias do tipo horizonte rolante e relax-and-fix também são consideradas. Consideramos também o cenário onde não conhecemos de antemão os itens da ordem de trabalho e o custo dos objetos, mas temos informações das distribuições de probabilidade de ambos. Nesse caso, apresentamos uma abordagem baseada em programação dinâmica aproximada para estimar a melhor estratégia a ser seguida em cada período. Comparamos os resultados obtidos pela PDA com os resultados encontrados por um método guloso. Em cenários adequados, os resultados mostram que a PDA consegue soluções superiores ao método guloso.

Palavras-chave: Problemas de corte multiperíodo, sobras aproveitáveis, otimização, horizonte rolante, relax-and-fix, programação dinâmica aproximada. 


\section{Abstract}

ROMÃO, O. C. Multi-period non-guillotine cutting problem with usable leftover. 2017. Doctoral Thesis - Institute of Mathematics and Statistics, University of São Paulo, São Paulo, 2017.

In this research, we study the multi-period two-dimensional cutting problem with usable leftover, which consists of cutting objects to produce a set of items. We assume a finite planning horizon with a finite amount of periods between the initial and final times. First we consider a deterministic version in which we know, a priori, the set of ordered items and the cost of the objects at each period. Some of the leftovers generated during the cutting process of the ordered items in a period may be used as objects in the future. The leftovers that can be used in the future are called usable leftovers. In general, a leftover is considered usable if it has dimensions equal to or greater than that of some item from a predefined list for the period. The goal is to minimize the total cost of the objects used to cut the set of ordered items of the entire considered horizon. If there are solutions with the same cost, we wish to find one that, at the end of the considered time horizon, maximizes the value of the remaining usable leftovers. We present a mathematical model of the problem using a bilevel formulation, which is transformed into a mixed integer linear programming model, due to the characteristics of the problem. Considering the difficulty in solving the developed model, we propose a heuristic approach based on approximate dynamic programming (ADP) to deal with the proposed problem. Other options based on the rolling horizon and relax-and-fix strategies are also considered. We also consider the scenario where we do not know in advance the set of ordered items and the cost of the objects, but we have information about the probability distributions of both. In this case, we present an approach based on approximate dynamic programming to estimate the best strategy to be followed at each period. We compared the results obtained by the ADP with the results found by a greedy method. In suitable scenarios, the results show that the ADP achieves superior solutions to the greedy method.

Keywords: Multi-period cutting problems, usable leftover, optimization, rolling horizon, relax-andfix, approximate dynamic programming. 


\section{Sumário}

1 Introdução $\quad 1$

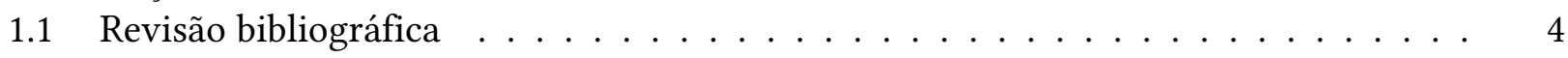

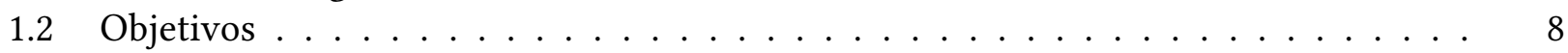

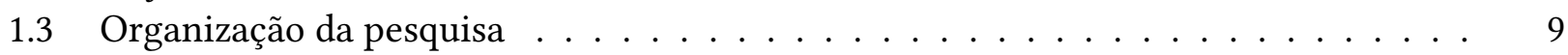

2 Definição e modelagem matemática do problema $\quad 11$

2.1 O problema de corte não-guilhotinado . . . . . . . . . . . . . . . . . 11

2.2 Reformulação para o problema multiperíodo . . . . . . . . . . . . . . . . 20

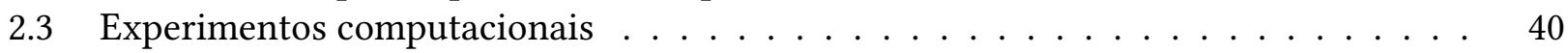

3 Métodos de solução $\quad 59$

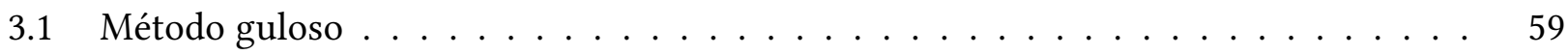

3.2 Horizonte rolante . . . . . . . . . . . . . . . . . . . 63

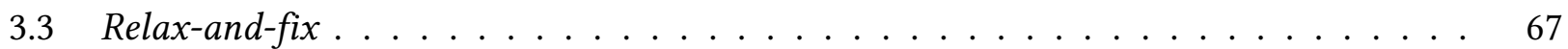

3.4 Programação dinâmica aproximada . . . . . . . . . . . . . . . . 68

3.4.1 Atualização das estimativas de utilização das sobras . . . . . . . . . . 75

3.4 .2 Critério de parada . . . . . . . . . . . . . . . 76

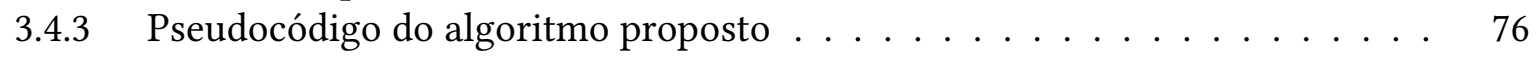

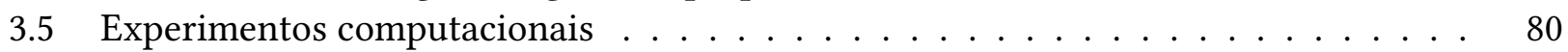

4 Planejamento sob incertezas $\quad 89$

4.1 PDA não-determinística . . . . . . . . . . . . . . . . . 90

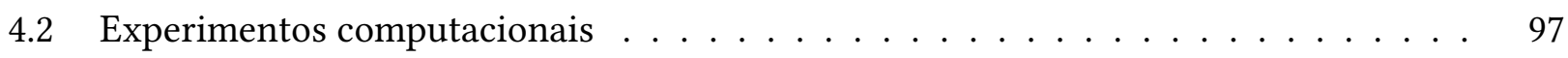

5 Conclusões $\quad 123$

$\begin{array}{ll}\text { A Instâncias determinísticas } & 126\end{array}$

$\begin{array}{ll}\text { Referências Bibliográficas } & 133\end{array}$ 


\section{Capítulo 1}

\section{Introdução}

Em um mundo cada vez mais competitivo, um dos principais desafios e objetivos das indústrias de manufatura é a redução do custo de produção. A minimização da quantidade de resíduos produzidos é de particular interesse nessas indústrias, uma vez que pequenas reduções de descartes podem resultar em grandes economias de matéria-prima e, com isso, reduzir consideravelmente o custo de produção. Essa economia pode ser transferida para o cliente, tornando a empresa mais competitiva no mercado, ou a economia pode ser transformada em lucro para a empresa.

Nesse contexto, com o propósito de economizar recursos, um dos problemas comumente encontrados nas indústrias de manufatura é o de como cortar peças maiores (objetos) visando a produção de peças menores (itens solicitados de uma ordem de trabalho) com dimensões específicas, otimizando um determinado objetivo. Tal problema é conhecido como problema de corte (do inglês, Cutting Problem), cuja finalidade é otimizar uma ou mais funções objetivo que podem ser, por exemplo, minimizar o número de objetos cortados, minimizar o custo dos objetos utilizados, minimizar a perda de material gerada durante o corte, etc. Esses problemas são essenciais no planejamento da produção de muitas indústrias e aparecem em diversos ambientes produtivos, tais como: indústrias de papel, vidro, metalurgia, plástica, têxtil, móveis, entre outras [8, 15, 19, 36, 37].

O problema de corte está diretamente relacionado ao problema de empacotamento [33]. O problema de corte pode ser entendido como o processo de cortar objetos para obter itens menores, enquanto o problema de empacotamento pode ser entendido como o processo de empacotar itens menores em objetos maiores. Dessa forma, considerando que um item cortado em uma posição pode ser visto como ocupando tal posição, para todo problema de corte existe um problema de empacotamento correspondente e vice-versa, ou seja, não existe diferença, em termos de estratégias de solução, entre os problemas de corte e empacotamento [23]. A diferença entre esses problemas ocorre, principalmente, na heterogeneidade dos itens [55]. Apesar da simplicidade da enunciação do problema, de forma geral, os problemas de corte e empacotamento são NP-Difíceis [24].

Uma característica do processo de corte de itens é que dificilmente encontraremos padrões de corte que utilizem todo o espaço do objeto. Dessa forma, ao realizar o processo de corte, sempre há a possibilidade de gerar restos de objetos (chamadas sobras) que podem ter dimensões suficientes para serem usados no corte de itens futuramente [2]. As sobras, dependendo de suas dimensões, podem ser 
consideradas como resíduos (que serão descartadas) ou sobras aproveitáveis. Uma sobra aproveitável, de um objeto cortado, pode ser definida como uma região retangular que possui dimensões iguais ou superiores a dimensões de referência. Tais sobras retangulares são consideradas aproveitáveis pois podem ser utilizadas futuramente como objetos. Toda sobra que não é aproveitável é considerada descarte [3].

Muitos trabalhos na literatura consideram o problema de corte sem analisar a sobra gerada no final do processo, descartando, dessa forma, soluções com sobras que podem ser utilizadas posteriormente. Visando o aproveitamento das sobras, Andrade et al. [6] propõe uma formulação matemática para o problema de corte bidimensional com o objetivo de minimizar o custo dos objetos cortados e dentre todas as soluções de custo mínimo, escolher aquela que maximize o valor das sobras aproveitáveis dos objetos usados. Entretanto, pode ocorrer que exista uma solução que seja um pouco mais "cara" que a escolhida, mas que gere uma sobra aproveitável de valor (ou dimensão) maior, minimizando, assim, o custo global do corte de objetos futuros. Por exemplo, suponha que tenhamos dois tipos de objetos disponíveis para o corte com dimensões $(10 \times 5)$ e $(12 \times 5)$ e, em um primeiro momento, temos que cortar um único item com dimensão $(6 \times 5)$. Supondo que o custo dos objetos é proporcional a sua área (para simplificar, suponha que cada unidade de área custa uma unidade monetária), com o objetivo de minimizar o custo dos objetos utilizados, a melhor solução é escolher o primeiro objeto. Assim, temos uma sobra de tamanho $(4 \times 5)$. Se, em um segundo momento, temos que cortar outro item de dimensão $(6 \times 5)$, a sobra que temos não pode ser aproveitada. Dessa forma, é necessário utilizar outro objeto com dimensão $(10 \times 5)$. Ao final desses dois períodos, teremos um gasto de 100 unidades monetárias e duas sobras não aproveitáveis com dimensões $(4 \times 5)$ cada. Agora, imagine que tenhamos escolhido para o corte do primeiro item o segundo objeto. Então, teremos um gasto de 60 unidades monetárias e uma sobra de tamanho $(6 \times 5)$, que é exatamente a dimensão do segundo item cortado. Assim, não precisamos utilizar um novo objeto, ou seja, uma economia de $40 \%$ em relação ao custo da primeira solução e sem termos sobras ao final desses dois períodos. Esta segunda solução poderia ser a escolhida caso tivéssemos, no primeiro período, alguma informação sobre os itens a serem cortados futuramente.

Uma primeira abordagem para resolver o problema de corte com sobras aproveitáveis, em um contexto multiperíodo, poderia consistir em formular o problema de cada período como um problema bi-objetivo, uma vez que o custo dos objetos utilizados e o valor das sobras podem ser considerados objetivos conflitantes. Nesse caso, teríamos várias soluções dominantes, conhecidas como soluções pareto-ótimas [34] e seríamos os responsáveis por escolher a solução que melhor se adaptaria às nossas necessidades, podendo ou não olhar os possíveis pedidos futuros. Na literatura, encontramos alguns trabalhos relacionados ao problema de corte bi-objetivo. Podemos citar, por exemplo, os trabalhos de Gradišar et al. [27], Armas et al. [7], Sardiñas et al. [47], Tiwari \& Chakraborti [52] e Miranda et al. [35].

A grande desvantagem da abordagem bi-objetivo é o fato de termos que ser capazes de construir a curva pareto e ainda deixarmos a cargo de alguém, por exemplo, o gerente de operações, onde o problema está sendo aplicado, escolher a solução que melhor se adapta às necessidades da empresa. Para isso, ele teria de analisar cada uma das soluções, que podem ser milhares, e selecionar 
a melhor baseado ou não nas informações que possui sobre os pedidos futuros. Tal tarefa pode ser árdua, demorada e, em alguns casos, impraticável. Em vista disso, propomos tratar o problema como multiperíodo e, dentre as possíveis soluções da curva pareto do período atual, escolher aquela que melhor se adapta às necessidades das ordens de trabalho futuras.

O presente trabalho está dividido em duas partes. Na primeira supomos que o problema é determinístico. Com determinístico queremos dizer que os itens solicitados em uma ordem de trabalho e o custo dos objetos disponíveis para a compra, em todos os períodos do horizonte considerado, são conhecidos à priori. Conhecendo todos os itens da ordem de trabalho antecipadamente, esses poderiam ser cortados de uma única vez para depois serem utilizados oportunamente. Sendo assim, o leitor poderia se perguntar “por que este é um problema multiperíodo?” A explicação está no fato de que, neste trabalho, consideramos que as limitações de tempo, mão-de-obra, disponibilidade de dinheiro para comprar matéria-prima e a manipulação do estoque permitem apenas processar os pedidos de um único período por vez. Na segunda parte do trabalho, consideramos um cenário mais realista do problema onde não conhecemos de antemão a ordem de trabalho dos itens e o custo dos objetos disponíveis para a compra de cada período, mas temos informações das distribuições de probabilidade de ambos.

Uma restrição bastante comum nos processos que envolvem o problema de corte bidimensional é exigir que os cortes se estendam em linha reta de um lado a outro do objeto. Tais cortes são chamados de cortes de guilhotina. Um padrão de corte é dito guilhotinado quando pode ser obtido a partir de cortes de guilhotina sucessivos. Na Figura 1.1a temos um padrão de corte não-guilhotinado e na Figura 1.1b um padrão de corte guilhotinado (a numeração indica uma possível ordem em que os cortes de guilhotina podem ser feitos). Neste trabalho, adotamos o padrão não-guilhotinado para o corte dos itens e não permitimos rotações dos itens dentro dos objetos.

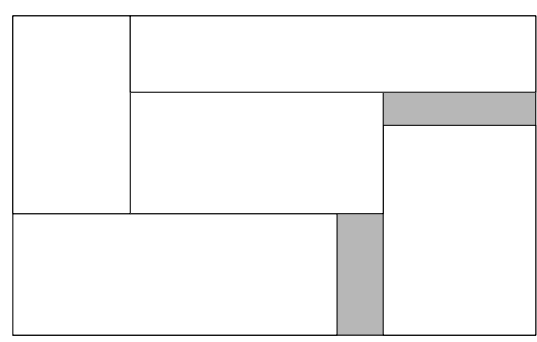

(a) Padrão de corte não-guilhotinado

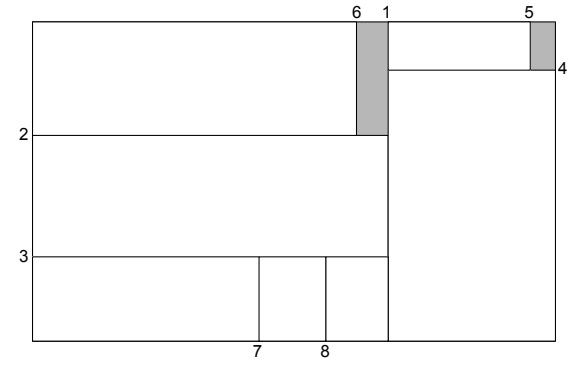

(b) Padrão de corte guilhotinado

Figura 1.1: Padrões de corte

Nesse contexto, o problema de corte bidimensional multiperíodo, tratado neste trabalho, consiste basicamente em resolver, a cada período de um horizonte de planejamento finito, um problema de corte bidimensional não-guilhotinado, para atender a ordem de trabalho dos itens solicitados em todos os períodos do horizonte de planejamento, porém não podendo antecipar a produção dos mesmos. As sobras de um objeto utilizado em um período ficam disponíveis no próximo período, tornando-se novos objetos, juntamente com os objetos disponíveis desse período. Consideramos que duas sobras podem ser produzidas a partir de cada objeto e que cada sobra é gerada por um simples corte guilhotinado, isto é, antes de cortar os itens de um objeto existem duas possibilidades: $(a)$ um 
corte guilhotinado vertical é feito de tal forma que o lado direito do objeto é uma sobra e então um corte guilhotinado horizontal é feito de forma que a parte superior do objeto é outra sobra; ou $(b)$ o corte guilhotinado horizontal é feito primeiro e em seguida é feito o corte guilhotinado vertical. A Figura 1.2 ilustra essas duas possibilidades. Desejamos satisfazer a ordem de trabalho dos itens minimizando a soma dos custos dos objetos utilizados (comprados) em todos os períodos e, dentre as diferentes possibilidades de se fazer isso, desejamos escolher aquela que maximize o valor das sobras aproveitáveis remanescentes ao final do horizonte de planejamento. Neste caso, o valor de uma sobra é dado por sua área multiplicado pelo custo por unidade de área do objeto. Denominamos este problema proposto de Problema de Corte Bidimensional Multiperíodo com Sobras Aproveitáveis (PCBMSA).

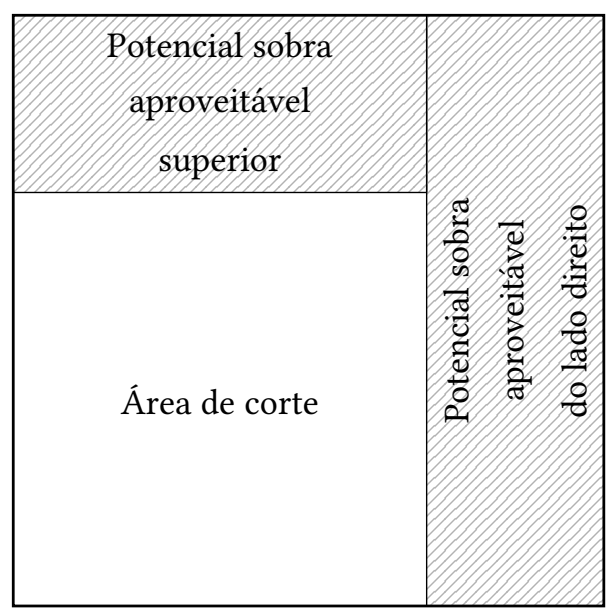

(a) Caso A

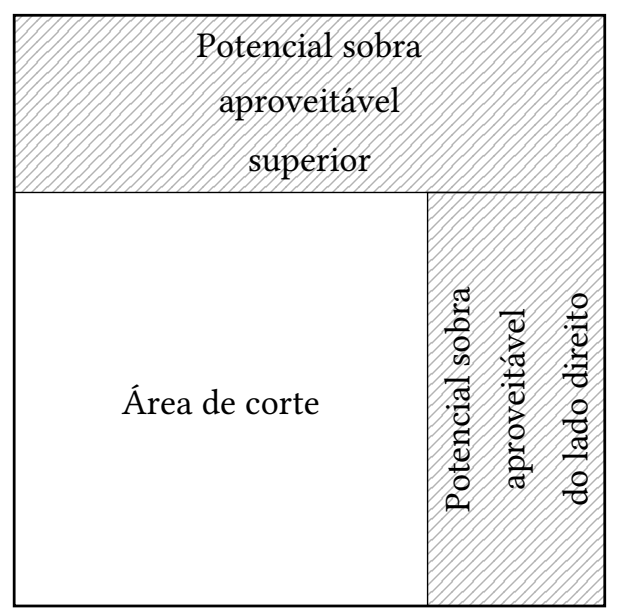

(b) Caso B

Figura 1.2: Representação das duas formas possíveis de gerar sobras a partir de um corte guilhotinado vertical e um horizontal. Extraída de Andrade et al. [6].

Considerando a grande quantidade de trabalhos na literatura sobre corte e empacotamento e a diversidade de nomes utilizados para um mesmo problema, Dyckhoff [23] elaborou uma tipologia, com o intuito de classificar os problemas de corte e empacotamento, baseada em quatro critérios principais: dimensionalidade, tipo de atribuição, características dos objetos e dos itens. De acordo com Dyckhoff [23], o problema abordado nesse trabalho pode ser classificado como 2/V/D/R. Posteriormente, Wäscher et al. [55] estendeu a tipologia apresentada por Dyckhoff [23] levando-se em consideração as dimensões e as formas dos objetos e itens, além disso, também foram definidas categorias para os tipos básicos de problemas de corte e empacotamento. Seguindo a tipologia de Wäscher et al. [55], nosso trabalho se encaixa na categoria Bin Packing Problem.

\subsection{Revisão bibliográfica}

Embora a literatura sobre problemas de corte seja bastante extensa, os trabalhos que abordam o aproveitamento de sobras são limitados, apesar de ser uma prática comum na indústria. O uso de aproveitamento de sobras em problemas de corte foi primeiramente citado por Brown [14]. Entretanto, os primeiros estudos relacionados a este assunto surgiram apenas após o trabalho de Dyckhoff 
[22], no qual o autor desenvolveu um modelo para o problema de corte unidimensional apontando a possibilidade deste modelo ser adaptado para utilizar as sobras dos objetos cortados. Vários outros trabalhos também abordam o problema de corte unidimensional com sobras aproveitáveis, dentre eles, podemos citar: Abuabara \& Morabito [2], Cherri et al. [15, 17], Chu \& Antonio [18], Cui \& Yang [20], Gradišar et al. [27, 28, 29], Gradišar \& Trkman [30], Roodman [46], Trkman \& Gradišar [53].

Em Roodman [46] são propostas heurísticas, baseadas na técnica de geração de colunas de Gilmore \& Gomory [25], para a geração de padrões para o problema de corte unidimensional em uma indústria de corte de aço. O objetivo principal é a minimização da perda de material, entretanto existe um objetivo secundário responsável por concentrar as sobras no menor número possível de padrões de cortes para serem utilizadas posteriormente no corte de itens.

No estudo de Scheithauer [48] é proposta uma estratégia para controlar as sobras geradas, chamadas de "comprimentos residuais" (residuals lengths), durante o processo de corte. O objetivo do trabalho é mostrar como as sobras podem ser consideradas utilizando a técnica de geração de colunas no modelo apresentado no trabalho de Gilmore \& Gomory [25]. Ao final, o autor também discute como estender o problema para o corte bidimensional.

Gradišar et al. [27] propõem um modelo matemático bi-objetivo e um procedimento heurístico para otimizar o corte de rolos em uma indústria de tecidos. O modelo bi-objetivo visa minimizar o número de itens que não são atendidos e minimizar a perda total (soma de todas as perdas inferiores a um limitante, por exemplo, o menor comprimento de todos os itens da ordem de trabalho). Caso a sobra fosse maior que um limitante ela retornaria para o estoque podendo ser usada futuramente. Além disso, os autores apresentam um procedimento heurístico guloso, chamado de COLA (COmputerized LAying out), baseado na resolução de múltiplos problemas da mochila. Posteriormente, uma modificação melhorada e generalizada do COLA foi proposta por Gradišar et al. [28], denominada de CUT. No estudo de Gradišar et al. [29] foi apresentada uma abordagem híbrida constituída de uma combinação de programação linear com um procedimento heurístico, baseado no algoritmo proposto por Gradišar et al. [27], para resolver o problema de corte com sobras. O objetivo da abordagem híbrida é atender a ordem de trabalho dos itens e acumular as possíveis sobras, que poderiam ser utilizadas futuramente, em um único objeto.

Chu \& Antonio [18] aplicam o problema de corte unidimensional com sobras aproveitáveis em uma indústria de corte de metais. O objetivo é minimizar a perda de material, o tempo para cortar as peças solicitadas e a sobra aproveitável. No trabalho, sobras que possuem um comprimento maior que um dado valor são consideradas sobras aproveitáveis. Caso contrário, são consideradas descartáveis (perda). Para resolver o problema, são propostos um modelo matemático não-linear e algoritmos baseados em programação dinâmica.

No trabalho de Gradišar \& Trkman [30] é desenvolvido um algoritmo, denominado C-CUT (Combined Cutting), que combina a heurística CUT (proposta por Gradišar et al. [28]) e um método exato. O objetivo é minimizar a perda de material gerada durante o corte dos itens, considerando que todos os objetos em estoque possuem comprimentos distintos. As sobras geradas durante o processo de corte, se forem suficientemente grandes, voltam para o estoque para serem reaproveitadas e não são consideras como perdas. 
No estudo feito em Cherri et al. [15] os autores apresentam modificações em heurísticas clássicas da literatura para o problema de corte unidimensional, de modo que as sobras geradas em cada padrão de corte sejam pequenas para serem descartadas como perdas ou suficientemente grandes para serem estocadas como sobras aproveitáveis, as quais seriam utilizadas posteriormente no corte de itens solicitados. No trabalho de Cherri et al. [16] as heurísticas propostas por Cherri et al. [15] são modificadas para que as sobras fiquem no estoque apenas por um tempo determinado. Dessa forma, as sobras têm prioridade no corte quando comparadas com os objetos disponíveis em estoque.

O problema de corte unidimensional com sobras aproveitáveis em uma empresa aeronáutica brasileira é estudado por Abuabara \& Morabito [2]. Em tal empresa, tubos estruturais metálicos são cortados para a produção de aeronaves agrícolas. Os autores propõem duas formulações matemáticas para resolver o problema com o objetivo de minimizar a perda, considerando que no estoque há diferentes tipos de objetos e possíveis sobras que foram geradas em cortes anteriores. O primeiro modelo é uma adaptação do modelo apresentado por Gradišar et al. [27], transformado em um problema de programação inteira mista. O segundo modelo é uma simplificação do primeiro.

Outro trabalho que visa resolver o problema de corte unidimensional com sobras aproveitáveis foi feito por Cui \& Yang [20]. Baseado no trabalho de Scheithauer [48], os autores apresentam um modelo matemático para o problema de corte levando em consideração as sobras aproveitáveis. Além disso, a quantidade de objetos no estoque e as sobras geradas durante o processo de corte são limitadas e os possíveis comprimentos das sobras são previamente definidos.

O estudo apresentado por Trkman \& Gradišar [53] considera o problema de corte unidimensional em que períodos consecutivos devem ser resolvidos com o objetivo de minimizar a perda ou o custo da produção. Todos os objetos em estoque têm comprimentos diferentes e as sobras geradas, caso sejam superiores a um determinado limitante, retornam para o estoque, podendo ser cortadas em um período seguinte.

Visando a indústria de papel, Poltroniere et al. [42] aborda os problemas de corte unidimensional e dimensionamento de lotes de forma integrada. No trabalho, os autores propõem um modelo matemático de otimização linear inteira mista, o qual é resolvido por heurísticas baseadas na relaxação lagrangiana da restrição de acoplamento. O objetivo é determinar quais e quantas bobinas devem ser produzidas em cada período do horizonte de planejamento, de forma a atender a demanda dos pedidos, evitar atrasos e estoques e minimizar os custos de produção, preparação, estoque e perda de material. Entretanto, as sobras geradas em um determinado período não são utilizadas nos períodos seguintes.

Poldi \& Arenales [41] propõem um modelo para o problema de corte unidimensional multiperíodo, cujo objetivo é minimizar o custo total dos objetos cortados em todos os períodos e o custo com estocagem de objetos e itens (que podem ou não ter sua produção antecipada). Entretanto, as possíveis sobras geradas não retornam para o estoque para serem reaproveitadas em cortes de itens nos períodos futuros. O modelo proposto pelos autores é resolvido de forma relaxada através de um método simplex com geração de colunas especializado para resolver o modelo. Como uma solução fracionária não é aplicável, duas abordagens para o arredondamento são propostas e testadas. As abordagens desenvolvidas são baseadas em horizonte rolante que, basicamente, consiste em tentar 
encontrar uma solução inteira apenas para o primeiro período e para os demais períodos a solução continua fracionária. Na próxima etapa, após o primeiro período ser resolvido, a lista de itens solicitados é atualizada, já que novos pedidos podem ser incluídos ou pedidos podem ser cancelados, e um novo problema de corte multiperíodo é resolvido. Os experimentos computacionais mostraram o ganho ao se adotar o problema de corte multiperíodo comparado com a solução lote-por-lote, tipicamente adotada.

Reinertsen \& Vossen [45] aborda o problema de corte unidimensional com data de entrega, ou seja, cada item deve ser produzido antes da data de entrega, caso contrário, é aplicada uma penalização (custo) pelo atraso na produção dos itens. O trabalho não considera a utilização das sobras geradas durante o corte. É proposto um modelo matemático, resolvido com geração de colunas, cujo objetivo é minimizar a soma dos atrasos na produção dos itens. Além de testar o modelo considerando os pedidos de todos os períodos, o modelo também é avaliado usando um framework de horizonte rolante proposto pelos autores.

Alguns trabalhos na literatura consideram o problema de corte bidimensional com sobras aproveitáveis, mas sem considerar a opção multiperíodo. Podemos citar, por exemplo, os trabalhos de Silva et al. [50], Andrade et al. [6] e Andrade et al. [4]. Nos trabalhos de Silva et al. [50] e Andrade et al. [4] estuda-se o problema de corte bidimensional multi-estágio (determinado pela quantidade de vezes que o objeto ou a serra devem ser rotacionados, fazendo cortes guilhotinados, para cortar todos os itens atribuídos ao objeto) com sobras aproveitáveis. Motivado pela indústria madeireira, Silva et al. [50] propõem um modelo de programação inteira mista para o problema de corte bidimensional dois-estágios e três-estágios, que é uma extensão do modelo proposto por Dyckhoff [22]. Em Andrade et al. [4] são apresentados dois modelos de otimização em dois níveis para o problema de corte bidimensional dois-estágios, que, devido às características do problema, são reformulados como um problema de um único nível, cujo objetivo é cortar os itens usando um conjunto de objetos de custo mínimo e, entre todas as possíveis soluções de custo mínimo, escolher aquela que maximize o valor das sobras aproveitáveis geradas durante o corte. Andrade et al. [6] propõem um modelo matemático multinível que aborda o problema de corte bidimensional não-guilhotinado com sobras aproveitáveis, cujo objetivo é o mesmo do apresentado por Andrade et al. [4]. A formulação também é estendida com o objetivo de minimizar o custo dos objetos e, entre todas as soluções de custo mínimo, escolher uma solução que maximiza o valor das sobras aproveitáveis e, entre elas, escolher aquela com menor número de sobras aproveitáveis. Como em Andrade et al. [4], o modelo multinível é reformulado como um problema de um único nível.

Por outro lado, Gramani \& França [31] e Poldi [40] analisam o problema de corte bidimensional multiperíodo, mas ambos não consideram o armazenamento das sobras geradas para serem utilizadas em períodos subsequentes do horizonte de planejamento. Em Gramani \& França [31], os autores combinam o problema de corte bidimensional com o problema de dimensionamento de lote. O objetivo é determinar a quantidade de itens produzidos em cada período do horizonte de planejamento, minimizando o número de objetos utilizados e os custos de armazenamento e de preparação. É apresentado um modelo matemático que é resolvido heuristicamente. A heurística proposta utiliza uma representação de uma rede de caminho mínimo. Cada arco da rede está associado a um problema de 
corte bidimensional capacitado, resolvido pelo método de geração de colunas proposto por Gilmore \& Gomory [26]. Depois de resolvido o problema de corte de todos os arcos, é resolvido um problema de caminho mínimo. Os resultados mostram que a solução, ao combinar os problemas, é $28 \%$ melhor que a obtida resolvendo cada um dos problemas separadamente. Poldi [40] considera a antecipação ou não da produção de alguns itens no horizonte de planejamento. Os objetos iniciais, que estão disponíveis em diferentes tamanhos e em quantidades limitadas, que não foram utilizadas em um dado período, são guardados e podem ser utilizados nos períodos seguintes. No trabalho é proposto um modelo de programação inteira com o objetivo de minimizar o custo das perdas e estocagem dos objetos e itens. O modelo é resolvido de forma relaxada através de geração de colunas. Além disso, são propostas duas heurísticas de arredondamento da solução baseadas em horizonte rolante.

No contexto de problemas de corte bidimensional multiperíodo com sobras aproveitáveis, aqui estudado e tipicamente empregado na indústria, encontramos apenas o trabalho de Silva et al. [51]. No trabalho, os autores integram o problema de corte bidimensional dois-estágios e o problema de dimensionamento de lotes. O objetivo é determinar a quantidade de itens que devem ser cortados dos objetos em cada período do horizonte de planejamento, decidindo se o corte de alguns itens deve ou não ser antecipado, de modo a minimizar os custos de produção e de armazenamento e os desperdícios dos objetos usados. Os objetos estão disponíveis em quantidade (virtualmente) infinita e todos possuem as mesmas dimensões. É proposto um modelo matemático para o problema integrado e supõe-se que todos os padrões de cortes possíveis são conhecidos à priori para cada período. As sobras geradas durante o processo de corte voltam para o estoque, podendo ser utilizadas em períodos posteriores. A principal diferença entre o nosso trabalho e o proposto por Silva et al. [51] está no fato de não precisarmos conhecer, à priori, os padrões de corte. Além disso, podemos destacar que nosso caso, consideramos que o catálogo dos objetos disponíveis para compra pode conter objetos com dimensões diferentes, mas com quantidades definidas; não permitimos a antecipação do corte dos itens; e adotamos o corte bidimensional não-guilhotinado ao invés do dois-estágios. Também consideramos um cenário não-determinístico do PCBMPSA, onde não conhecemos, à priori, a ordem de trabalho dos itens solicitados e o custo dos objetos disponíveis para a compra de cada período, temos apenas informações das distribuições de probabilidade de ambos.

\subsection{Objetivos}

O objetivo geral deste trabalho é propor métodos para resolver o problema de corte não-guilhotinado multiperíodo com sobras aproveitáveis, o qual consiste em cortar os itens de uma ordem de trabalho de todos os períodos usando um conjunto de objetos de custo mínimo e, entre todas as possíveis soluções de custo mínimo, escolher aquela que maximize o valor das sobras aproveitáveis remanescentes ao final do horizonte de planejamento. Nesse contexto, devemos definir quais objetos disponíveis serão comprados e utilizados; decidir o tipo de corte (Caso A ou Caso B, veja Figura 1.2) de cada objeto definindo, assim, as dimensões das sobras de cada objeto; utilizar, caso necessário, as sobras geradas dos objetos de um determinado período em períodos posteriores como novos objetos; definir os itens a serem cortados de cada objeto; e determinar onde (posição) cada item deve ser cortado no objeto ao 
qual foi designado. Para isso, é proposta uma formulação matemática para o problema abordado neste trabalho, aqui denominado, Problema de Corte Bidimensional Multiperíodo com Sobras Aproveitáveis (PCBMPSA). Também propomos uma abordagem para lidar com um cenário não-determinístico do PCBMPSA. Nesse caso, apresentamos uma abordagem para resolver o problema quando temos apenas informações das distribuições de probabilidade da ordem de trabalho dos itens e o custo dos objetos.

Especificamente, pretendemos:

- Propor um modelo para o PCBMPSA;

- Propor e aplicar uma abordagem baseada em programação dinâmica aproximada (PDA) e outros métodos heurísticos para o PCBMPSA;

- Comparar o desempenho da abordagem baseada em PDA e dos métodos heurísticos propostos com o modelo;

- Apresentar resultados que comprovem a viabilidade da utilização do PDA e dos métodos heurísticos;

- Propor uma estratégia para resolver um cenário mais realista do PCBMPSA, onde não conhecemos de antemão os itens solicitados de uma ordem de trabalho e o custo dos objetos disponíveis para a compra de cada período (versão não-determinística do problema), mas temos informações das distribuições de probabilidade de ambos;

- Mostrar em quais cenários a PDA consegue bons resultados para a versão não-determinística do problema;

- Comprovar que a PDA consegue melhores resultados que uma estratégia gulosa para o caso não-determinístico do problema.

\subsection{Organização da pesquisa}

O presente trabalho está organizado como segue. No Capítulo 2 apresentamos o modelo proposto em [6], que representa o problema tratado nesse trabalho, mas considerando apenas um período. Também introduzimos o modelo proposto para o caso multiperíodo, assim como alguns exemplos ilustrativos. No Capítulo 3 fazemos uma descrição de alguns métodos heurísticos que podem ser utilizados para a resolução do PCBMPSA e apresentamos alguns experimentos numéricos comparando as estratégias heurísticas propostas. No Capítulo 4 descrevemos um cenário não-determinístico do PCBMPSA e propomos uma abordagem baseada em programação dinâmica aproximada para resolvê-lo. Finalmente, apresentamos as conclusões e delineamos alguns possíveis trabalhos futuros no Capítulo 5. 


\section{Capítulo 2}

\section{Definição e modelagem matemática do problema}

O problema de corte bidimensional abordado no presente capítulo, consiste em minimizar o custo dos objetos utilizados para o corte de um conjunto de itens retangulares solicitados de uma ordem de trabalho, e entre todas as soluções de custo mínimo, escolher a que maximize o valor das sobras remanescentes. Nesse caso, o valor das sobras é dado pela sua área multiplicado pelo custo por unidade de área do objeto. Tipicamente, na indústria, uma instância deste problema é considerada no início de cada período do horizonte de planejamento, o que pode resultar em soluções de baixa qualidade, ou seja, com alto desperdício quando considerado um conjunto de períodos de planejamento. Com o objetivo de abordar essa característica, é proposto neste capítulo um modelo matemático para o problema de corte bidimensional multiperíodo com sobras aproveitáveis (PCBMPSA), que é baseado na formulação proposta por Andrade et al. [6]. Para entendermos o modelo proposto para o PCBMPSA, primeiramente, apresentamos um modelo para o problema de corte bidimensional com sobras aproveitáveis (com um único período) proposto por Andrade et al. [6]. Em seguida, estendemos este modelo para a versão multiperíodo. A principal diferença entre a abordagem apresentada neste capítulo e as encontradas na literatura (por exemplo, [4, 6, 31, 40,50]) é o fato de abordarmos a versão multiperíodo do problema de corte que permite o aproveitamento das sobras dos objetos, podendo ser utilizadas em períodos futuros, o que pode minimizar o custo e o desperdício.

\subsection{O problema de corte não-guilhotinado com pré-corte gui- lhotinado ${ }^{1}$}

Nesta seção, apresentamos o modelo para o problema de corte bidimensional não-guilhotinado com sobras aproveitáveis (PCSA) proposto por Andrade et al. [6]. Primeiramente, apresentamos um modelo para o problema de corte não-guilhotinado que não considera sobras aproveitáveis. Em seguida, introduzimos o conceito de sobras aproveitáveis e uma formulação que minimiza a soma dos custos

\footnotetext{
${ }^{1}$ Esta seção está fortemente baseada no trabalho de Andrade et al. [6].
} 
dos objetos utilizados e, dentre todas as soluções de custo mínimo, escolhe uma que maximiza o valor das sobras aproveitáveis.

Dado um conjunto de $n$ itens a serem cortados (itens da ordem de trabalho) com largura $w_{i}$ e altura $h_{i}, i=1, \ldots, n$, e um conjunto de $m$ objetos disponíveis com largura $W_{j}$, altura $H_{j}$ e custo $c_{j}$ por unidade de área, $j=1, \ldots, m$, o problema de corte, abordado nesta seção, tem como objetivo cortar os itens da ordem de trabalho utilizando os objetos disponíveis minimizando o custo dos objetos usados. Não permitimos rotações e não consideramos outras restrições relacionadas ao posicionamento dos itens dentro dos objetos, ou o tipo de cortes nos objetos, por exemplo, corte guilhotinado ou cortes em estágios. Consideramos que os cortes dos objetos são infinitamente finos, que as dimensões dos itens e objetos são inteiras e que o custo por unidade de área dos objetos é inteiro e não-negativo.

Seja $u_{j} \in\{0,1\}, j=1, \ldots, m$, indicando se o objeto $j$ é usado para cortar ao menos um item da ordem de trabalho $\left(u_{j}=1\right)$ ou não $\left(u_{j}=0\right)$, e seja $v_{i j} \in\{0,1\}, i=1, \ldots, n, j=1, \ldots, m$, para atribuir o item $i$ ao objeto $j$ (neste caso, $v_{i j}=1$ ) ou não $\left(v_{i j}=0\right)$ ). É fácil perceber que essas variáveis devem satisfazer as seguintes relações

$$
u_{j} \geq v_{i j}, i=1, \ldots, n, j=1, \ldots, m
$$

e que as variáveis $v_{i j}$ devem satisfazer as restrições

$$
\sum_{j=1}^{m} v_{i j}=1, i=1, \ldots, n
$$

para garantir que cada item seja atribuído a exatamente um único objeto. Além disso, também já podemos definir a função objetivo a ser minimizada como

$$
\sum_{j=1}^{m} c_{j} W_{j} H_{j} u_{j}
$$

Se definimos $\left(x_{i}, y_{i}\right), i=1, \ldots, n$, como a coordenada do centro Cartesiano do item $i$, podemos facilmente escrever restrições que garantem que o item $i$ deve estar contido dentro do objeto $j$ sempre que $v_{i j}=1$. Se, sem perda de generalidade, arbitrariamente supomos que o canto esquerdo-inferior dos objetos está localizado na origem do plano bidimensional, essas restrições podem ser escritas como

$$
\begin{array}{lll}
x_{i}-w_{i} / 2 \geq 0, & i=1, \ldots, n \\
y_{i}-h_{i} / 2 \geq 0, & i=1, \ldots, n \\
x_{i}+w_{i} / 2 \leq W_{j}+\left(\hat{W}-W_{j}\right)\left(1-v_{i j}\right), & i=1, \ldots, n, j=1, \ldots, m \\
y_{i}+h_{i} / 2 & \leq H_{j}+\left(\hat{H}-H_{j}\right)\left(1-v_{i j}\right), & i=1, \ldots, n, j=1, \ldots, m
\end{array}
$$

onde $\hat{W}=\max _{j=1, \ldots, m}\left\{W_{j}\right\}$ e $\hat{H}=\max _{j=1, \ldots, m}\left\{H_{j}\right\}$. Note que o conjunto de restrições (2.4) representa a 
reformulação big- $M$ de

$$
v_{i j}=1 \Longrightarrow\left(x_{i}, y_{i}\right) \in\left[w_{i} / 2, W_{j}-w_{i} / 2\right] \times\left[h_{i} / 2, H_{j}-h_{i} / 2\right], i=1, \ldots, n, j=1, \ldots, m .
$$

Para evitar a sobreposição entre itens $i$ e $i^{\prime}$ atribuídos a um mesmo objeto $j$, podemos escrever as restrições

$$
\begin{aligned}
v_{i j}=v_{i^{\prime} j}=1 \Longrightarrow\left|x_{i}-x_{i^{\prime}}\right| \geq\left(w_{i}+w_{i^{\prime}}\right) / 2 \text { ou }\left|y_{i}-y_{i^{\prime}}\right| \geq\left(h_{i}+h_{i^{\prime}}\right) / 2, & \\
i & =1, \ldots, n, i^{\prime}=i+1, \ldots, n, j=1, \ldots, m .
\end{aligned}
$$

Reescrevendo " $|a| \geq b$ " como " $a \geq b$ ou $a \leq-b$ " e definindo as variáveis auxiliares $\pi_{i i^{\prime}}, \tau_{i i^{\prime}} \in$ $\{0,1\}, i=1, \ldots, n, i^{\prime}=i+1, \ldots, n$, para a reformulação big- $M$ dos quatro termos das disjunções, obtemos as seguintes restrições

$$
\begin{array}{r}
x_{i^{\prime}}+w_{i^{\prime}} / 2 \leq x_{i}-w_{i} / 2+\hat{W}\left(1-v_{i j}\right)+\hat{W}\left(1-v_{i^{\prime} j}\right)+\hat{W} \pi_{i i^{\prime}}+\hat{W} \tau_{i i^{\prime}}, \\
x_{i^{\prime}}-w_{i^{\prime}} / 2 \geq x_{i}+w_{i} / 2-\hat{W}\left(1-v_{i j}\right)-\hat{W}\left(1-v_{i^{\prime} j}\right)-\hat{W} \pi_{i i^{\prime}}-\hat{W}\left(1-\tau_{i i^{\prime}}\right), \\
y_{i^{\prime}}+h_{i^{\prime}} / 2 \leq y_{i}-h_{i} / 2+\hat{H}\left(1-v_{i j}\right)+\hat{H}\left(1-v_{i^{\prime} j}\right)+\hat{H}\left(1-\pi_{i i^{\prime}}\right)+\hat{H} \tau_{i i^{\prime}}, \\
y_{i^{\prime}}-h_{i^{\prime}} / 2 \geq y_{i}+h_{i} / 2-\hat{H}\left(1-v_{i j}\right)-\hat{H}\left(1-v_{i^{\prime} j}\right)-\hat{H}\left(1-\pi_{i i^{\prime}}\right)-\hat{H}\left(1-\tau_{i i^{\prime}}\right), \\
i=1, \ldots, n, i^{\prime}=i+1, \ldots, n, j=1, \ldots, m .
\end{array}
$$

Os dois primeiros " $M$-termos" de cada restrição servem para neutralizar o efeito das restrições sempre que $v_{i j}=0$ ou $v_{i^{\prime} j}=0$, o que significa que o item $i$ ou o item $i^{\prime}$ não foi atribuído ao objeto $j$ e, portanto, não há sobreposição entre eles no objeto $j$. Os outros dois “ $M$-termos" de cada restrição, junto com as quatro possíveis combinações dos valores das variáveis binárias $\pi_{i i^{\prime}}$ e $\tau_{i i^{\prime}}$, modelam as disjunções que garantem que o item $i^{\prime}$ deve estar "à esquerda", "à direita", "acima” ou "abaixo" do item $i$ com uma distância mínima para evitar sobreposição, sempre que os itens $i$ e $i^{\prime}$ forem atribuídos ao mesmo objeto.

Como mostrado em [5], é possível perceber que a troca da posição de dois itens $i$ e $i^{\prime}$ idênticos ( $w_{i}=w_{i^{\prime}}$ e $h_{i}=h_{i^{\prime}}$ ) gera uma solução simétrica. Para evitar essa situação, que pode fazer com que um algoritmo branch-and-bound demore mais tempo para finalizar, restrições que evitam sobreposições de um par de itens idênticos podem ser modeladas de uma forma diferente. Em termos gerais, se os itens $i$ e $i^{\prime}, \operatorname{com} i^{\prime}>i$, são idênticos, ao invés de exigir que o item $i^{\prime}$ esteja "à esquerda", "à direita", "acima" ou "abaixo" do item $i$, é suficiente exigir que o item $i^{\prime}$ esteja "à direita" ou "acima" do item $i$. Sem perda de generalidade, suponha que os itens idênticos estão numerados consecutivamente, que existem $\tilde{n}$ tipos diferentes de itens e que há $z_{q}$ itens do $q$-ésimo tipo, $q=1, \ldots, \tilde{n}$, com $\sum_{q=1}^{\tilde{n}} z_{q}=n$. Denotando $o_{q}=\sum_{q^{\prime}=1}^{q-1} z_{q^{\prime}}$, temos que os itens do $q$-ésimo tipo estão numerados de $o_{q}+1$ a $o_{q}+z_{q}$. Portanto, as restrições de não sobreposição podem ser reescritas como 


$$
\begin{aligned}
& x_{i^{\prime}}-w_{i^{\prime}} / 2 \geq x_{i}+w_{i} / 2-\hat{W}\left(1-v_{i j}\right)-\hat{W}\left(1-v_{i^{\prime} j}\right)-\hat{W} \pi_{i i^{\prime}}, \\
& y_{i^{\prime}}-h_{i^{\prime}} / 2 \geq y_{i}+h_{i} / 2-\hat{H}\left(1-v_{i j}\right)-\hat{H}\left(1-v_{i^{\prime} j}\right)-\hat{H}\left(1-\pi_{i i^{\prime}}\right), \\
& q=1, \ldots, \tilde{n}, i=o_{q}+1, \ldots, o_{q}+z_{q}, i^{\prime}=i+1, \ldots, o_{q}+z_{q}, j=1, \ldots, m .
\end{aligned}
$$

e

$$
\begin{aligned}
& x_{i^{\prime}}+w_{i^{\prime}} / 2 \leq x_{i}-w_{i} / 2+\hat{W}\left(1-v_{i j}\right)+\hat{W}\left(1-v_{i^{\prime} j}\right)+\hat{W} \pi_{i i^{\prime}}+\hat{W} \tau_{i i^{\prime}}, \\
& x_{i^{\prime}}-w_{i^{\prime}} / 2 \geq x_{i}+w_{i} / 2-\hat{W}\left(1-v_{i j}\right)-\hat{W}\left(1-v_{i^{\prime} j}\right)-\hat{W} \pi_{i i^{\prime}}-\hat{W}\left(1-\tau_{i i^{\prime}}\right), \\
& y_{i^{\prime}}+h_{i^{\prime}} / 2 \leq y_{i}-h_{i} / 2+\hat{H}\left(1-v_{i j}\right)+\hat{H}\left(1-v_{i^{\prime} j}\right)+\hat{H}\left(1-\pi_{i i^{\prime}}\right)+\hat{H} \tau_{i i^{\prime}}, \\
& y_{i^{\prime}}-h_{i^{\prime}} / 2 \geq y_{i}+h_{i} / 2-\hat{H}\left(1-v_{i j}\right)-\hat{H}\left(1-v_{i^{\prime} j}\right)-\hat{H}\left(1-\pi_{i i^{\prime}}\right)-\hat{H}\left(1-\tau_{i i^{\prime}}\right), \\
& q=1, \ldots, \tilde{n}, i=o_{q}+1, \ldots, o_{q}+z_{q}, i^{\prime}=o_{q}+z_{q}+1, \ldots, n, j=1, \ldots, m .
\end{aligned}
$$

O conjunto de restrições (2.5) modela a não sobreposição entre itens idênticos usando um número bem menor de restrições e variáveis binárias, com o recurso adicional de evitar o tipo de soluções simétricas mencionado anteriormente. Como introduzido anteriormente, o conjunto de restrições (2.6) modela a não sobreposição entre pares de itens diferentes.

O problema de corte, em resumo, pode ser modelado minimizando a função objetivo (2.3) nas variáveis $u_{j} \in\{0,1\}(j=1, \ldots, m), v_{i j} \in\{0,1\}(i=1, \ldots, n, j=1, \ldots, m), x_{i}, y_{i} \in \mathbb{R}(i=$ $1, \ldots, n), \pi_{i i^{\prime}} \in\{0,1\}\left(i=1, \ldots, n, i^{\prime}=i+1, \ldots, n\right)$ e $\tau_{i i^{\prime}} \in\{0,1\}\left(q=1, \ldots, \tilde{n}, i=o_{q}+\right.$ $\left.1, \ldots, o_{q}+z_{q}, i^{\prime}=i+1, \ldots, n\right)$ sujeita à $(2.1,2.2,2.4,2.5,2.6)$. Chamamos este modelo de $\mathcal{C}$.

Como um exemplo ilustrativo, considere uma instância com $m=2$ objetos idênticos com $W_{1}=$ $W_{2}=12$ e $H_{1}=H_{2}=20$ e $c_{1}=c_{2}=1$; e $n=4$ itens diferentes com $w_{1}=w_{2}=w_{3}=w_{4}=5$ e $h_{1}=16, h_{2}=14, h_{3}=12$ e $h_{4}=8$. A Figura 2.1 exibe duas soluções viáveis diferentes com custo 480. Já que a soma das áreas dos quatro itens é maior que a área de qualquer um dos objetos, ao menos dois objetos são necessários para cortar os itens e, portanto, as soluções viáveis apresentadas são ótimas. Entretanto, existe uma característica que diferencia essas duas soluções ótimas e que não está sendo considerada no modelo apresentado acima. Considerando que o resultado de um primeiro corte guilhotinado pode ser guardado como um pedaço residual a ser usado futuramente, a solução da Figura 2.1b tem uma sobra guilhotinada em um dos objetos (área hachurada) que pode ser potencialmente usada no corte de dois itens do tipo $4\left(\operatorname{com} w_{4}=5\right.$ e $\left.h_{4}=8\right)$, enquanto a solução da Figura 2.1a não possui essa propriedade.

Supondo que duas sobras guilhotinadas podem ser produzidas a partir de cada objeto usado (objetos não usados não podem gerar sobras residuais) e que cada sobra é gerada por um simples corte guilhotinado, isto é, antes de cortar os itens de um objeto existem duas possibilidades: (a) um corte guilhotinado vertical é feito de tal forma que o lado direito do objeto é uma sobra e então um corte guilhotinado horizontal é feito de forma que a parte superior do objeto é outra sobra; ou (b) o corte guilhotinado horizontal é feito primeiro e em seguida é feito o corte guilhotinado vertical. A Figura 2.2 ilustra os dois casos, chamados, daqui em diante, de Caso A e Caso B, respectivamente. É importante notar que, embora a soma da área das sobras sejam as mesmas, a soma do seus valores podem ser diferentes. Em ambos os casos, uma lista contendo $d \geq 1$ itens catalogados com larguras 
$\bar{w}_{1}, \ldots, \bar{w}_{d}$ e alturas $\bar{h}_{1}, \ldots, \bar{h}_{d}$ é considerada como um dado de entrada e uma sobra é considerada aproveitável se ela pode conter pelo menos um item do catálogo. Note que a lista de itens catalogados pode coincidir com a lista de itens da ordem de trabalho (neste caso, temos $d=n$ e $\bar{w}_{i}=w_{i}$ e $\bar{h}_{i}=h_{i}$, para $i=1, \ldots, n$ ) ou pode consistir de um único item representando a largura mínima $\bar{w}_{1}$ e a altura mínima $\bar{h}_{1}$ que uma sobra deve ter para ser considerada aproveitável. Vale destacar que a definição de sobra apresentada neste parágrafo exclui qualquer outra parte do objeto, que será considerada desperdício no processo de corte.

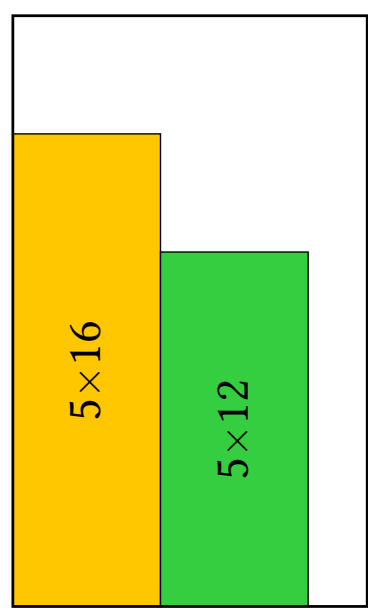

$12 \times 20$

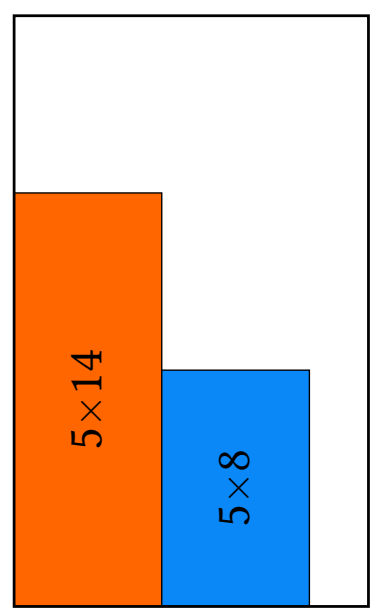

$12 \times 20$

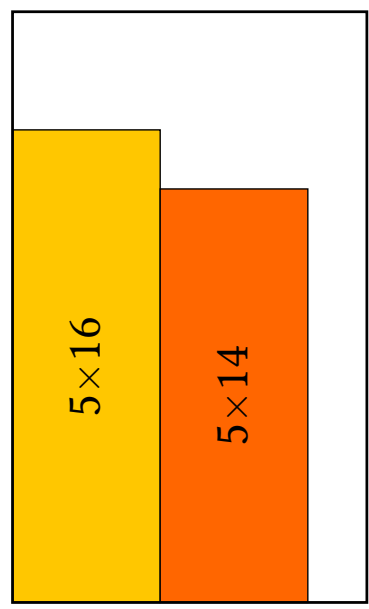

$12 \times 20$

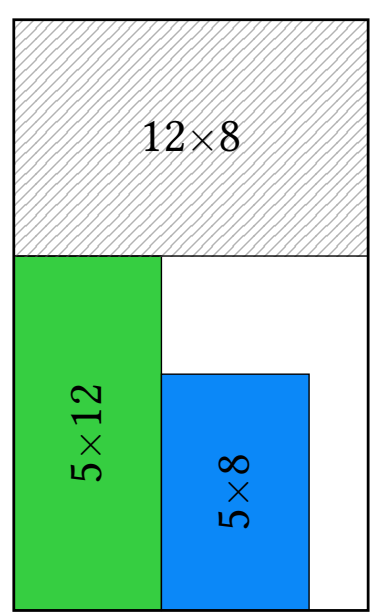

$12 \times 20$

(a)

(b)

Figura 2.1: Representação de duas soluções alternativas ótimas de uma instância simples com dois objetos e quatro itens. Considerando as sobras, a solução (b) produzirá uma sobra da qual dois itens do tipo 4 (com $w_{4}=5$ e $\left.h_{4}=8\right)$ podem ser cortados no futuro. Extraída de Andrade et al. [6].

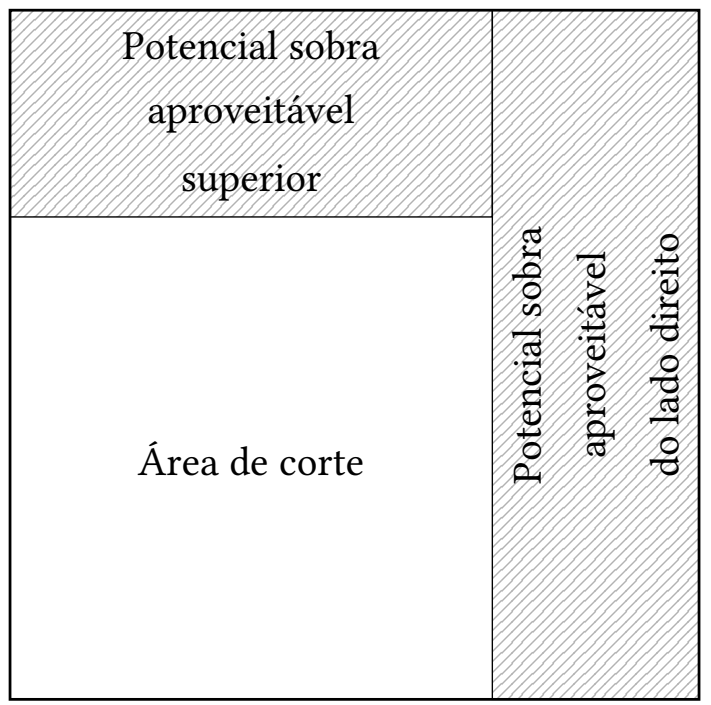

(a) Caso A

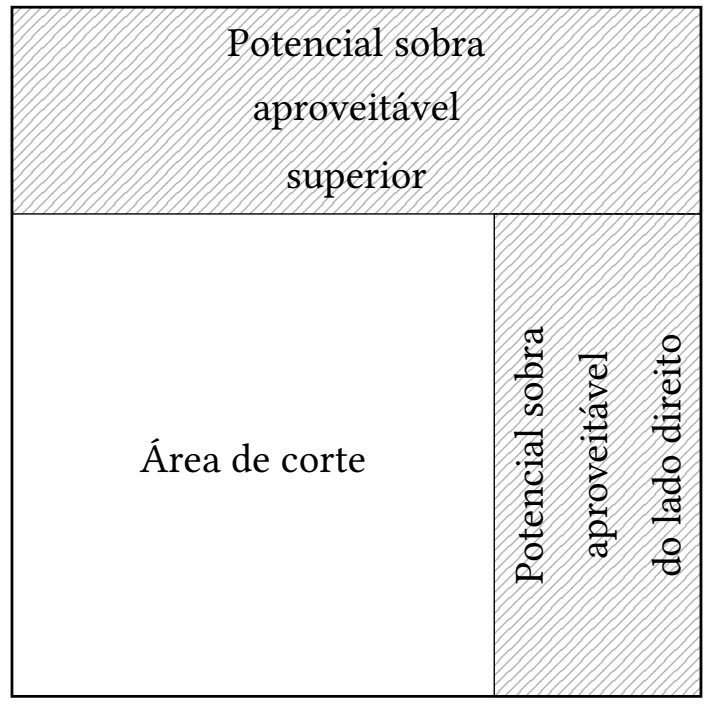

(b) Caso B

Figura 2.2: Representação das duas formas possíveis de gerar sobras guilhotinadas aproveitáveis a partir de um corte guilhotinado vertical e um horizontal. A Figura 2.2a representa o caso onde o corte guilhotinado vertical é feito primeiro, enquanto a Figural 2.2b representa o caso onde o corte guilhotinado horizontal é feito primeiro. Extraída de Andrade et al. [6]. 
Definimos $t_{j}$ como a variável associada à altura da sobra superior do objeto $j$ e $r_{j}$ como a variável associada a largura da sobra do lado direito do objeto $j$, para $j=1, \ldots, m$. Lembre-se de que objetos não usados não podem gerar sobras, assim, temos que $t_{j}=r_{j}=0$ sempre que $u_{j}=0$. No Caso $A$, a largura e a altura da sobra superior são dadas por $W_{j}-r_{j}$ e $t_{j}$, respectivamente, enquanto a sobra do lado direito tem largura dada por $r_{j}$ e altura fixa dada por $H_{j}$. No Caso B, a sobra superior tem uma largura fixa dada por $W_{j}$ e altura dada por $t_{j}$, enquanto a sobra do lado direito tem largura e altura dadas por $r_{j}$ e $H_{j}-t_{j}$, respectivamente. Definimos o valor da sobra como o valor por unidade de área do objeto $\left(c_{j}, j=1, \ldots, m\right)$ vezes a área da sobra, sempre que a sobra puder conter ao menos um item do catálogo, ou zero, caso contrário. Portanto, dado $t_{j}$ e $r_{j}, j=1, \ldots, m$, a soma dos valores das sobras é dado por

$$
\sum_{j=1}^{m} c_{j} \max \left\{\alpha_{j}^{t}+\alpha_{j}^{r}, \beta_{j}^{t}+\beta_{j}^{r}\right\},
$$

onde, $\alpha_{j}^{t}$ e $\alpha_{j}^{r}\left(\beta_{j}^{t}\right.$ e $\left.\beta_{j}^{r}\right)$ estão associados, respectivamente, a área da sobra superior e a sobra da direita no padrão de corte do Caso A (Caso B). Assim, devemos ter que:

$$
\begin{aligned}
& \alpha_{j}^{t}=\left\{\begin{aligned}
\left(W_{j}-r_{j}\right) t_{j}, & \text { se existe } 1 \leq s \leq d \text { tal que } W_{j}-r_{j} \geq \bar{w}_{s} \text { e } t_{j} \geq \bar{h}_{s} \\
0, & \text { caso contrário, }
\end{aligned}\right. \\
& \alpha_{j}^{r}=\left\{\begin{aligned}
r_{j} H_{j}, & \text { se existe } 1 \leq s \leq d \text { tal que } r_{j} \geq \bar{w}_{s} \text { e } H_{j} \geq \bar{h}_{s} \\
0, & \text { caso contrário }
\end{aligned}\right. \\
& \beta_{j}^{t}=\left\{\begin{aligned}
W_{j} t_{j}, & \text { se existe } 1 \leq s \leq d \text { tal que } W_{j} \geq \bar{w}_{s} \text { e } t_{j} \geq \bar{h}_{s}, \\
0, & \text { caso contrário, }
\end{aligned}\right. \\
& \beta_{j}^{r}=\left\{\begin{aligned}
r_{j}\left(H_{j}-t_{j}\right), & \text { se existe } 1 \leq s \leq d \text { tal que } r_{j} \geq \bar{w}_{s} \text { e } H_{j}-t_{j} \geq \bar{h}_{s} \\
0, & \text { caso contrário, }
\end{aligned}\right. \\
& j=1, \ldots, m \text {. }
\end{aligned}
$$

A fim de combinar as sobras guilhotinadas com o problema de corte apresentado anteriormente, é suficiente substituir o conjunto de restrições (2.4), que garante que cada um dos itens deve ser colocado dentro do objeto para o qual foi atribuído, por um conjunto de restrições modificado que garante que cada um dos itens deve ser colocado dentro da "Área de corte" (veja a Figura 2.2) do objeto ao qual ele foi atribuído. Tal conjunto de restrições é dado por

$$
\begin{array}{lll}
x_{i}-w_{i} / 2 \geq 0, & i=1, \ldots, n \\
y_{i}-h_{i} / 2 \geq 0, & i=1, \ldots, n \\
x_{i}+w_{i} / 2 \leq W_{j}-r_{j}+\left(\hat{W}-W_{j}\right)\left(1-v_{i j}\right), & i=1, \ldots, n, j=1, \ldots, m \\
y_{i}+h_{i} / 2 & \leq H_{j}-t_{j}+\left(\hat{H}-H_{j}\right)\left(1-v_{i j}\right), & i=1, \ldots, n, j=1, \ldots, m
\end{array}
$$

e

$$
\begin{aligned}
0 & \leq t_{j} \leq H_{j} u_{j}, \quad j=1, \ldots, m \\
0 & \leq r_{j} \leq W_{j} u_{j}, \quad j=1, \quad
\end{aligned}
$$


As restrições (2.10) garantem que a altura da sobra superior e a largura da sobra do lado direito são não-negativas e que elas devem ser nulas sempre que o objeto não for usado.

Com o que foi apresentado, agora podemos apresentar o primeiro modelo do problema de corte com sobras aproveitáveis, o qual consiste em cortar os itens da ordem de trabalho usando um conjunto de objetos de custo mínimo e, entre todas as possíveis soluções de custo mínimo, escolher uma que maximize o valor das sobras aproveitáveis. Assim, o modelo é dado pela minimização de (2.7) nas variáveis $\alpha_{j}^{t}, \alpha_{j}^{r}, \beta_{j}^{t}, \beta_{j}^{r}, t_{j}, r_{j}, u_{j}, v_{i j}, \pi_{i i^{\prime}}$ e $\tau_{i i^{\prime}}$ sujeita à (2.8) e sujeita à $t_{j}, r_{j}, u_{j}, v_{i j}, \pi_{i i^{\prime}}$ e $\tau_{i i^{\prime}}$ ser solução da minimização de (2.3) sujeita à (2.1, 2.2, 2.9, 2.10, 2.5, 2.6). Este tipo de problema de otimização, no qual existe uma restrição que requer que um subconjunto de variáveis seja uma solução de um outro problema de otimização, é conhecido como Problema de Programação em Dois Níveis [21].

Para construir um modelo Programação Linear Inteira Mista (MIP, do inglês Mixed Integer Programming) dois níveis, do problema descrito acima, precisamos eliminar: $(i)$ o termo $\max \left\{\alpha_{j}^{t}+\right.$ $\left.\alpha_{j}^{r}, \beta_{j}^{t}+\beta_{j}^{r}\right\}$ que aparece na função objetivo (2.7), (ii) as "condições se" que aparecem na definição de $\alpha_{j}^{t}$, $\alpha_{j}^{r}$, $\beta_{j}^{t}$ e $\beta_{j}^{r}$ em (2.8), e (iii) os termos não-lineares $r_{j} t_{j}$ que aparecem na definição de $\alpha_{j}^{t}$ e $\beta_{j}^{r}$ em (2.8).

Definindo as variáveis contínuas $\gamma_{j}$ e as variáveis binárias $\eta_{j} \in\{0,1\}$, para $j=1, \ldots, m$, e adicionando as restrições

$$
\begin{aligned}
\gamma_{j} & \leq \alpha_{j}^{t}+\alpha_{j}^{r}+W_{j} H_{j} \eta_{j} \\
\gamma_{j} & \leq \beta_{j}^{t}+\beta_{j}^{r}+W_{j} H_{j}\left(1-\eta_{j}\right)
\end{aligned} \quad j=1, \ldots, m
$$

a função objetivo (2.7) pode ser reescrita como uma função linear

$$
\sum_{j=1}^{m} c_{j} \gamma_{j}
$$

É fácil perceber que a variável binária $\eta_{j}$ força $\gamma_{j}$ a ser menor ou igual a $\alpha_{j}^{t}+\alpha_{j}^{r}$ ou menor ou igual a $\beta_{j}^{t}+\beta_{j}^{r}$, e que $W_{j} H_{j}$ em (2.11) desempenha o papel de big-M na formulação MIP dessa disjunção. Já que a função objetivo (2.12) é maximizada, temos que $\gamma_{j}=\max \left\{\alpha_{j}^{t}+\alpha_{j}^{r}, \beta_{j}^{t}+\beta_{j}^{r}\right\}$, para $j=1, \ldots, m$, em uma solução, conforme desejado.

Considerando que as dimensões dos itens e dos objetos são inteiros positivos, pode-se mostrar que $r_{j}$ e $t_{j}, j=1, \ldots, m$, assumem valores inteiros na solução (veja, por exemplo, [10, 11]). Para modelar o produto $r_{j} t_{j}$ usando restrições MIP, usamos um truque bem conhecido, substituímos uma das variáveis por sua representação binária, reduzindo o produto a uma soma de produtos de variáveis inteiras e binárias, para o qual existe uma representação MIP (veja, por exemplo, [32, 56]). Seja $\theta_{j \ell} \in\{0,1\}, j=1, \ldots, m, \ell=1, \ldots,\left\lfloor\log _{2}\left(H_{j}\right)\right\rfloor+1$. Note que $H_{j}$ é um limitante superior para os possíveis valores que $t_{j}$ pode assumir e que $\left\lfloor\log _{2}\left(H_{j}\right)\right\rfloor+1$ é um limite superior para o número de bits necessário para a representação binária de $t_{j}, j=1, \ldots, m$. Portanto, temos que cada $t_{j}$ pode ser escrito como 


$$
t_{j}=\sum_{\ell=1}^{\left\lfloor\log _{2}\left(H_{j}\right)\right\rfloor+1} 2^{\ell-1} \theta_{j \ell}, \quad j=1, \ldots, m,
$$

e, assim, cada produto $r_{j} t_{j}$ pode ser escrito como

$$
r_{j} t_{j}=\sum_{\ell=1}^{\left\lfloor\log _{2}\left(H_{j}\right)\right\rfloor+1} 2^{\ell-1} r_{j} \theta_{j \ell}, \quad j=1, \ldots, m .
$$

Dessa forma, o produto de duas variáveis inteiras é reduzido à soma dos produtos de uma variável inteira e uma variável binária. O valor de cada um desses produtos é igual a zero quando a variável binária é igual a zero e coincide com o valor da variável inteira quando a variável binária é igual a um. Essa situação pode ser modelada usando restrições MIP considerando as variáveis contínuas $\omega_{j \ell}$, $j=1, \ldots, m, \ell=1, \ldots,\left\lfloor\log _{2}\left(H_{j}\right)\right\rfloor+1$, tais que

$$
\omega_{j \ell}=\left\{\begin{array}{cl}
r_{j}, & \text { sempre que } \theta_{j \ell}=1, \quad j=1, \ldots, m, \ell=1, \ldots,\left\lfloor\log _{2}\left(H_{j}\right)\right\rfloor+1, \\
0, & \text { caso contrário, }
\end{array}\right.
$$

o que pode ser modelado adicionando as seguintes restrições MIP

$$
\begin{aligned}
0 & \leq \omega_{j \ell} \leq r_{j}, \\
r_{j}-\left(1-\theta_{j \ell}\right) W_{j} & \leq \omega_{j \ell} \leq \theta_{j \ell} W_{j},
\end{aligned}
$$

Assim, temos que toda variável $t_{j}$ pode ser substituída pela sua representação binária (2.13) e todo produto $r_{j} t_{j}$ (nas definições de $\alpha_{j}^{t}$ e $\beta_{j}^{r}$ em (2.8)) pode ser substituído por sua reformulação MIP dada por

$$
r_{j} t_{j}=\sum_{\ell=1}^{\left\lfloor\log _{2}\left(H_{j}\right)\right\rfloor+1} 2^{\ell-1} \omega_{j \ell}, \quad j=1, \ldots, m .
$$

Para modelar (2.8) como restrições MIP, sejam $\bar{\alpha}_{i j}^{t}, \bar{\alpha}_{i j}^{r}, \bar{\beta}_{i j}^{t}, \bar{\beta}_{i j}^{r} \in\{0,1\}, i=1, \ldots, d, j=1, \ldots, m$, e considere as restrições:

$$
\begin{array}{rlrl}
\bar{w}_{i} \leq W_{j}-r_{j}+\hat{W}\left(1-\bar{\alpha}_{i j}^{t}\right) & \text { e } & \bar{h}_{i} \leq t_{j}+\hat{H}\left(1-\bar{\alpha}_{i j}^{t}\right), \\
\bar{w}_{i} \leq r_{j}+\hat{W}\left(1-\bar{\alpha}_{i j}^{r}\right) & \text { e } & \bar{h}_{i} \leq H_{j}+\hat{H}\left(1-\bar{\alpha}_{i j}^{r}\right), \\
\bar{w}_{i} \leq W_{j}+\hat{W}\left(1-\bar{\beta}_{i j}^{t}\right) & \mathrm{e} & \bar{h}_{i} \leq t_{j}+\hat{H}\left(1-\bar{\beta}_{i j}^{t}\right), \\
\bar{w}_{i} \leq r_{j}+\hat{W}\left(1-\bar{\beta}_{i j}^{r}\right) & \mathrm{e} & \bar{h}_{i} \leq & H_{j}-t_{j}+\hat{H}\left(1-\bar{\beta}_{i j}^{r}\right), \\
& & & i=1, \ldots, d, j=1, \ldots, m,
\end{array}
$$




$$
\begin{aligned}
& 0 \leq \alpha_{j}^{t} \leq W_{j} t_{j}-r_{j} t_{j} \quad \text { e } \quad \alpha_{j}^{t} \leq W_{j} H_{j} \sum_{i=1}^{d} \bar{\alpha}_{i j}^{t}, \\
& 0 \leq \alpha_{j}^{r} \leq r_{j} H_{j} \quad \text { e } \quad \alpha_{j}^{r} \leq W_{j} H_{j} \sum_{i=1}^{d} \bar{\alpha}_{i j}^{r} \text {, } \\
& 0 \leq \beta_{j}^{t} \leq W_{j} t_{j} \quad \text { e } \quad \beta_{j}^{t} \leq W_{j} H_{j} \sum_{i=1}^{d} \bar{\beta}_{i j}^{t} \text {, } \\
& 0 \leq \beta_{j}^{r} \leq r_{j} H_{j}-r_{j} t_{j} \quad \text { e } \quad \beta_{j}^{r} \leq W_{j} H_{j} \sum_{i=1}^{d} \bar{\beta}_{i j}^{r}, \\
& j=1, \ldots, m \text {. }
\end{aligned}
$$

Note que, em (2.16) e (2.17), $t_{j}$ deve ser substituída por sua representação binária (2.13) e $r_{j} t_{j}$ deve ser substituído por sua representação MIP (2.15) (por questão de simplicidade, optamos em não substituí-los no texto). O conjunto de restrições (2.16) garante que as variáveis $\bar{\alpha}_{i j}^{t}, \bar{\alpha}_{i j}^{r}$, $\bar{\beta}_{i j}^{t}$ e $\bar{\beta}_{i j}^{r}$ tomem valor zero quando o $i$-ésimo item do catálogo não cabe dentro da sobra superior do Caso A, na sobra do lado direito do Caso A, na sobra superior do Caso B e na sobra do lado direito do Caso B, respectivamente. Caso o item caiba dentro da sobra, as variáveis ficam livres e podem assumir valor zero ou um. Dessa forma, o conjunto de restrições (2.17) garante que as variáveis $\alpha_{j}^{t}, \alpha_{j}^{r}$, $\beta_{j}^{t}$ e $\beta_{j}^{r}$ serão nulas quando na sobra correspondente não couber nenhum item do catálogo. Caso contrário, elas ficam livres para assumir qualquer valor entre zero e a área da sobra correspondente. Uma vez que essas variáveis limitam o crescimento de $\gamma_{j}$ em (2.11), a função objetivo (2.12) é uma combinação linear não-negativa de $\gamma_{j}$, e o problema de nível superior é um problema de maximização, numa solução, as variáveis $\alpha_{j}^{t}, \alpha_{j}^{r}, \beta_{j}^{t}$ e $\beta_{j}^{r}$ atingirão seus valores máximos possíveis, que coincidirão com a área das sobras aproveitáveis.

Dessa forma, nosso modelo MIP de dois níveis consiste em maximizar (2.12) nas variáveis $u_{j}, v_{i j}$, $x_{i}, y_{i}, \pi_{i i^{\prime}}, \tau_{i i^{\prime}}, r_{j}, \theta_{j \ell}, \omega_{j \ell}, \gamma_{j}, \eta_{j}, \alpha_{j}^{t}, \alpha_{j}^{r}, \beta_{j}^{t}, \beta_{j}^{r}, \bar{\alpha}_{i j}^{t}, \bar{\alpha}_{i j}^{r}, \bar{\beta}_{i j}^{t}$ e $\bar{\beta}_{i j}^{r}$ sujeita a $(2.11,2.14,2.16,2.17)$ e sujeita à $u_{j}, v_{i j}, x_{i}, y_{i}, \pi_{i i^{\prime}}, \tau_{i i^{\prime}}, r_{j}$ e $\theta_{j \ell}$ ser uma solução de minimizar (2.3) sujeita à $(2.1,2.2,2.9,2.10,2.5,2.6)$.

Como as funções objetivos (2.12) e (2.3) do problema do nível superior e inferior, respectivamente, assumem apenas valores inteiros e possuem limitantes superiores e inferiores triviais, podemos reformular o modelo dois níveis como um modelo MIP. Assim, a função objetivo do problema do nível superior pode ser usada, em escala apropriada, como desempate entre todas as possíveis soluções ótimas do problema de nível inferior. Essa reformulação é possível para o modelo dois níveis do problema de corte com sobras aproveitáveis porque é um problema particular de dois níveis no qual as soluções do problema do nível inferior não dependem dos valores das variáveis do problema do nível superior.

É fácil perceber que a função objetivo (2.12) assume valores inteiros que são maiores ou iguais a zero e estritamente menores que $\sum_{j=1}^{m} c_{j} W_{j} H_{j}$. Assim, a combinação das funções objetivos (2.12) e (2.3) dada por

$$
\left(\sum_{j=1}^{m} c_{j} W_{j} H_{j}\right)\left(\sum_{j=1}^{m} c_{j} W_{j} H_{j} u_{j}\right)-\sum_{j=1}^{m} c_{j} \gamma_{j}
$$

desempenha o papel desejado. Note que o termo relacionado à função objetivo (2.3) aparece multiplicado por um limitante superior da função objetivo (2.12). Isso implica que cada unidade do termo 
relacionado com a função objetivo (2.3) é mais importante que todo valor do termo relacionado à função objetivo (2.12). Assim, o termo relacionado com a função objetivo (2.12) participa no desempate das soluções de custo mínimo, escolhendo uma com máximo valor de sobras aproveitáveis. Portanto, a reformulação MIP do problema de corte com sobras aproveitáveis (PCSA) é dada por minimizar (2.18) nas variáveis $u_{j}, v_{i j}, x_{i}, y_{i}, \pi_{i i^{\prime}}, \tau_{i i^{\prime}}, r_{j}, \theta_{j \ell}, \omega_{j \ell}, \gamma_{j}, \eta_{j}, \alpha_{j}^{t}, \alpha_{j}^{r}, \beta_{j}^{t}, \beta_{j}^{r}, \bar{\alpha}_{i j}^{t}, \bar{\alpha}_{i j}^{r}, \bar{\beta}_{i j}^{t}$ e $\bar{\beta}_{i j}^{r}$ sujeita a $(2.1,2.2,2.5,2.6,2.9,2.10,2.11,2.14,2.16,2.17)$.

\subsection{Reformulação para o problema multiperíodo}

Como dito anteriormente, muitos trabalhos na literatura consideram o problema de corte sem analisar a sobra gerada durante o processo de corte, descartando soluções com sobras que podem ser utilizadas futuramente. A formulação apresentada na seção anterior trata do problema de corte bidimensional com sobras aproveitáveis. Porém, em um contexto multiperíodo, para um dado instante, pode ocorrer que tenhamos uma solução com um custo um pouco maior que o custo da solução escolhida, mas que gere sobras aproveitáveis de valores (ou dimensões) maiores e que podem ser melhores utilizadas posteriormente, minimizando, assim, o custo global. Neste contexto, nessa seção apresentamos uma formulação para o problema de corte (não-guilhotinado) multiperíodo com sobras (guilhotinadas) aproveitáveis (PCBMPSA) que consiste, basicamente, em resolver, para cada período, um problema de corte bidimensional atendendo a ordem de trabalho dos itens do período, podendo usar como objetos sobras aproveitáveis de períodos anteriores.

Para ilustrar o problema abordado neste trabalho, suponha que temos uma instância com os dados apresentados na Figura 2.3. Considere que cada unidade de área dos objetos custe uma unidade monetária e suponha que toda sobra que possa conter, no mínimo, um item de tamanho $3 \times 1$ seja considerada aproveitável. A Figura 2.4 ilustra três possíveis soluções. Lembre-se de que, neste trabalho, consideramos que cada objeto pode gerar, no máximo, duas sobras, como mostrado na Figura 1.2. Resolvendo o problema de corte para cada período de forma separada, sem analisar os itens solicitados nas ordens de trabalho futuras, encontramos a solução 1 (ilustrada na Figura 2.4a), a qual utiliza três objetos (gerando um custo de 77) e gera três sobras finais (com soma dos valores igual a 35). Como os pedidos futuros não são considerados, no primeiro período é utilizado o objeto $O_{11}$ que possui custo de 1 unidade a menos que o objeto $O_{12}$ e, assim, no terceiro período será preciso utilizar o objeto $O_{31}$ para cortar o item desse período, uma vez que não existe sobra (proveniente de algum período anterior) que possa conter tal item. No entanto, se no primeiro período utilizarmos o objeto $O_{12}$, mesmo tendo um gasto um pouco maior neste período, ele irá gerar sobras que serão melhor utilizadas nos cortes do itens solicitados nos próximos períodos, como é mostrado nas soluções 2 e 3 (Figuras $2.4 \mathrm{~b}$ e $2.4 \mathrm{c}$, respectivamente), o que minimizará o custo global dos objetos utilizados nos três períodos.

Observe que as soluções 2 e 3 (apresentadas, respectivamente, pelas Figuras 2.4b e 2.4c) possuem o mesmo custo global dos objetos utilizados (53), mas a soma dos valores das sobras finais são diferentes (10 e 11, respectivamente). Essa diferença acontece porque no segundo período da solução 2 (Figura 2.4b), os itens $4 \times 5$ e $3 \times 1$ são cortados no objeto $O_{21}$, gerando um descarte de dimensão $1 \times 1$ 
e uma sobra aproveitável de dimensão $3 \times 1$ neste objeto, enquanto na solução 3 (Figura 2.4c) o item $3 \times 1$ é cortado em uma sobra gerada no período anterior, não gerando nenhuma área de desperdício. Como dito, o objetivo do problema é encontrar uma solução que minimiza a soma dos custos dos objetos utilizados em todos os períodos do horizonte de planejamento e, entre as soluções de custo mínimo, escolher uma que maximize a soma dos valores das sobras aproveitáveis remanescentes. Dessa forma, para o nosso problema, a solução 3 é melhor que a solução 2.

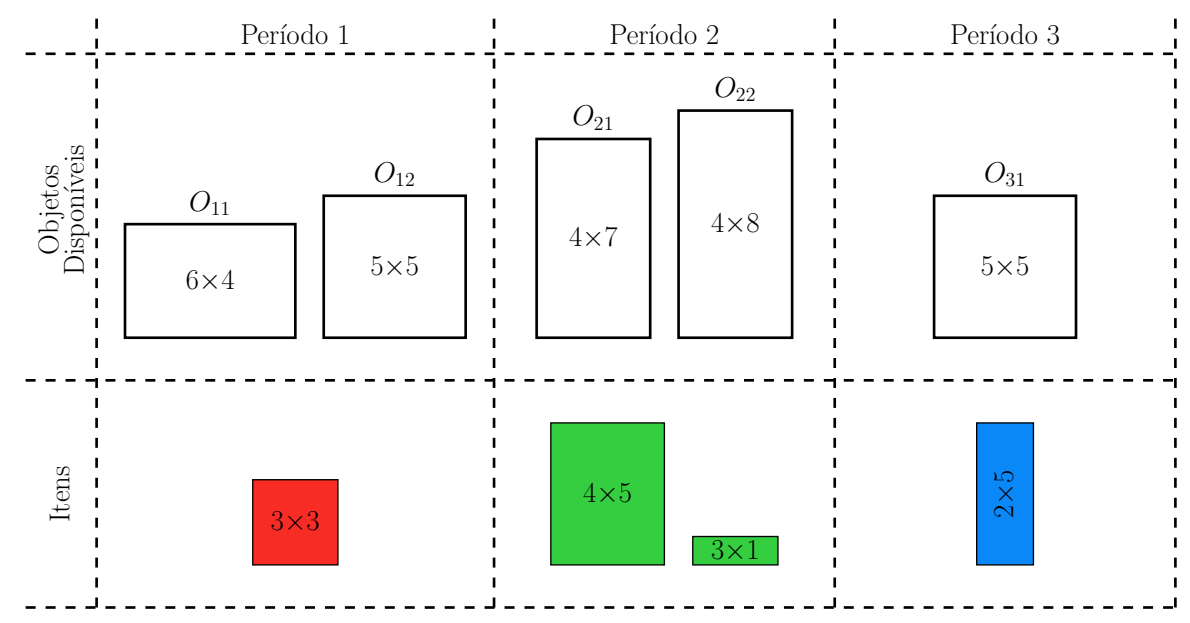

Figura 2.3: Dados de uma instância para o problema de corte bidimensional multiperíodo com sobras aproveitáveis. Considere que cada unidade de área dos objetos custe uma unidade monetária.

Como podemos notar, as sobras que não foram utilizadas em um período ficam disponíveis nos períodos posteriores, como é mostrado, por exemplo, na Figura 2.4a na qual a sobra $s r_{11}$ não é utilizada em nenhum período futuro e, ao fim do terceiro período, é considerada como sobra remanescente. Por outro lado, na mesma figura, a sobra $s t_{11}$ é utilizada no corte de um item do segundo período. Além disso, note que uma sobra também pode gerar até duas sobras aproveitáveis que poderão ser utilizadas futuramente ou serem consideradas sobras remanescentes, como acontece na sobra $s t_{12}$ na solução 3 (Figura 2.4c), que é utilizada no segundo período e gera uma nova sobra aproveitável. Já os objetos disponíveis para compra, em um determinado período, e que não foram comprados, não ficam mais disponíveis nos próximos períodos. Por exemplo, o objeto $O_{12}$ da solução mostrada pela Figura 2.4a não foi comprado e, assim, não fica disponível nos períodos seguintes.

O problema de corte multiperíodo, sem considerar o aproveitamento das sobras dos objetos, pode ser descrito como: dado um intervalo de períodos (horizonte de planejamento) iniciando no período $p$ e terminando no período $P$, de forma que o tamanho do horizonte de planejamento é $P-p+1$ períodos; um número $m_{s}$ de objetos disponíveis em cada período $s$, para $s=p, p+1, \ldots, P-1, P$, com largura $W_{s j}$, altura $H_{s j}$ e custo $c_{s j}$ por unidade de área, $s=p, \ldots, P, j=1, \ldots, m_{s}$; e um número $n_{s}$ de itens da ordem de trabalho do período $s, s=p, \ldots, P$, com largura $w_{s i}$ e altura $h_{s i}$, $s=p, \ldots, P, i=1, \ldots, n_{s}$; o objetivo é cortar todos os itens das ordens de trabalho de cada período, utilizando os objetos disponíveis, minimizando o custo dos objetos usados. Como no PCSA, rotações não são permitidas, não há restrições relacionadas ao posicionamento dos itens dentro dos objetos, os cortes dos objetos são infinitamente finos, as dimensões dos itens e objetos são inteiras e o custo por unidade de área dos objetos é inteiro e não-negativo. 


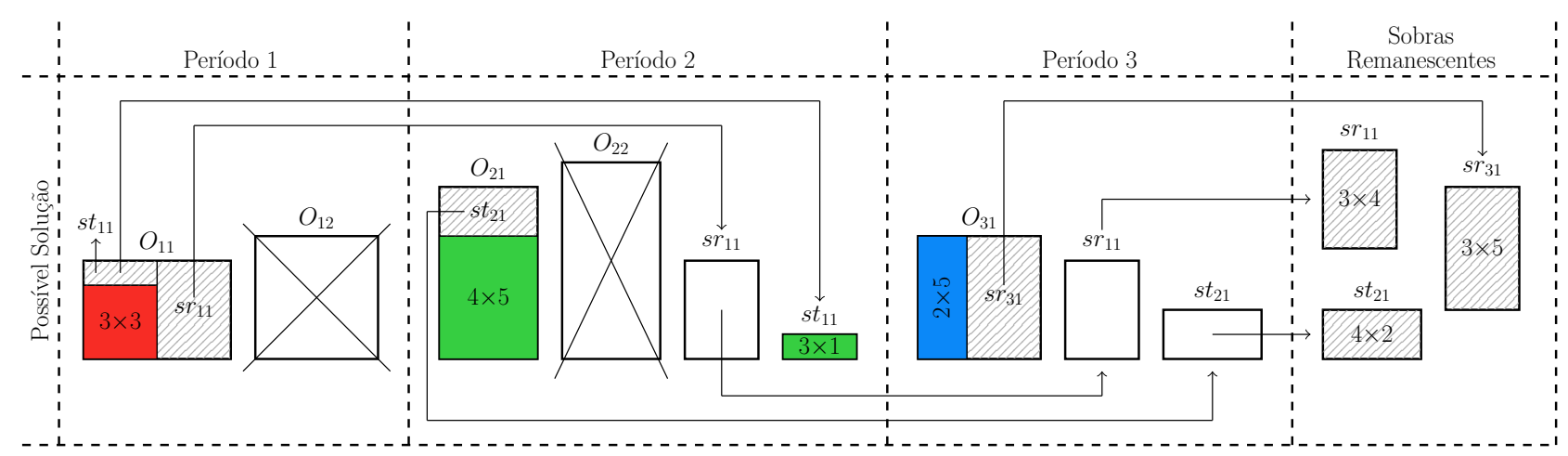

(a) Solução 1 - solução gulosa que minimiza o custo de utilização dos objetos de cada período, de forma separada, sem analisar as ordens de trabalho dos itens futuras. Custo dos objetos utilizados igual a 77 e valor das sobras remanescentes igual a 35.

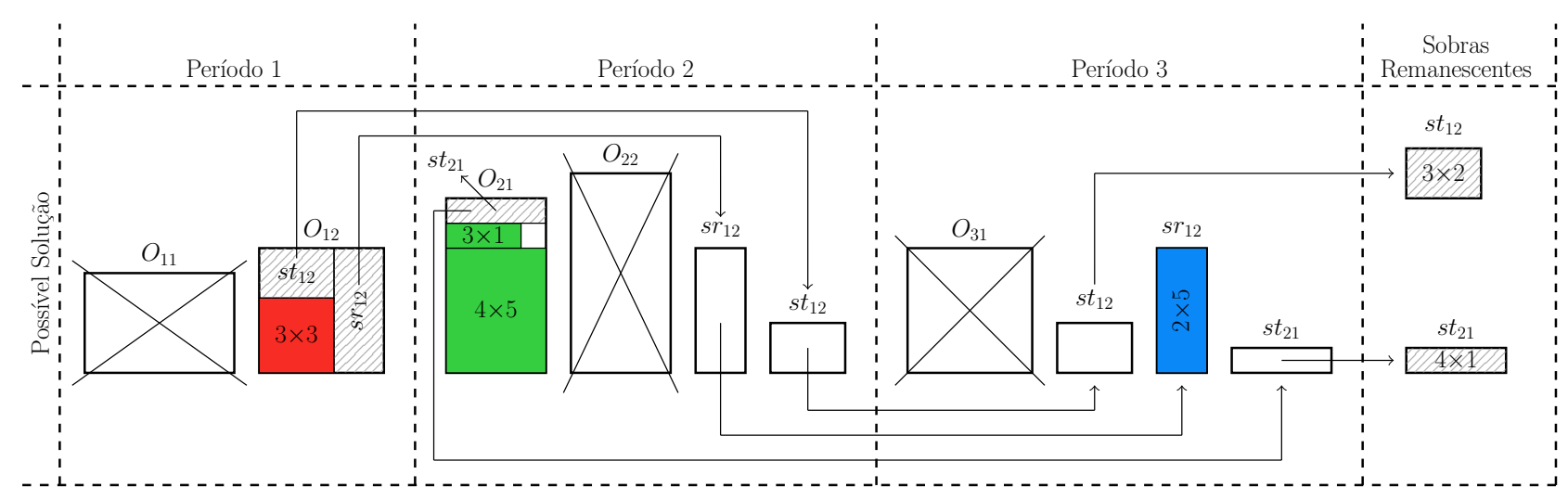

(b) Solução 2 - solução de custo global mínimo de utilização dos objetos, mas com uma área de desperdício no objeto $O_{21}$. Custo dos objetos utilizados igual a 53 e valor das sobras remanescentes igual a 10 .

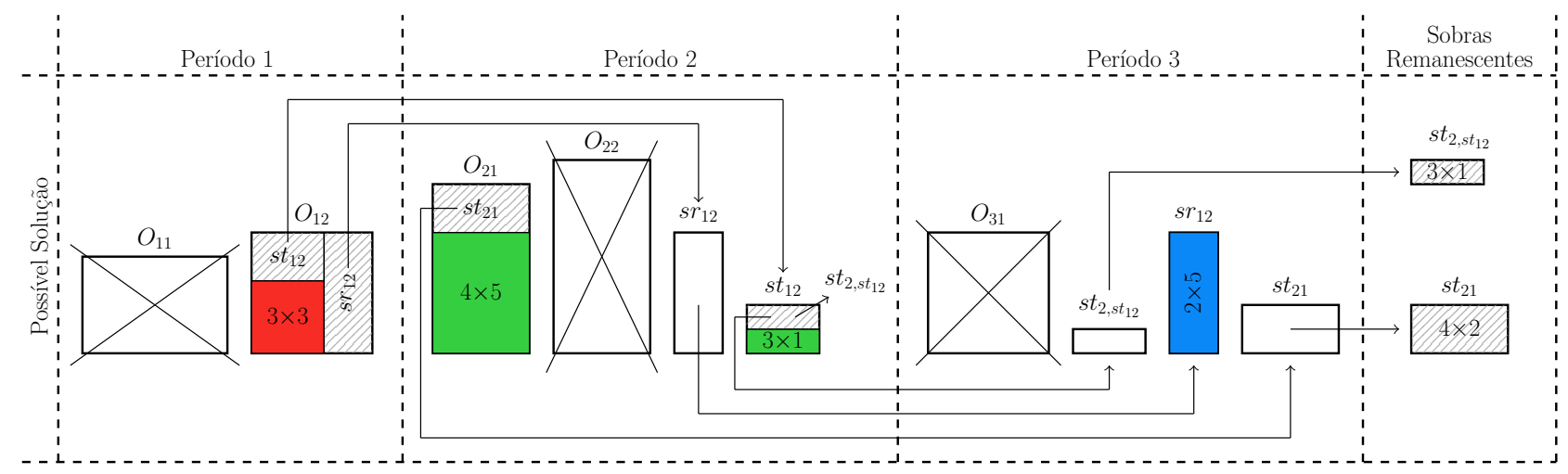

(c) Solução 3 - solução de custo global mínimo de utilização dos objetos e com valor máximo das sobras remanescentes. Custo dos objetos utilizados igual a 53 e valor das sobras remanescentes igual a 11.

Figura 2.4: Três possíveis soluções para a instância com os dados apresentados na Figura 2.3. 
Como mostramos na Figura 2.4, em cada período, os objetos usados podem gerar sobras aproveitáveis, que podem ser usadas em um período posterior ao qual o objeto foi cortado, tornando-se novos objetos disponíveis nesse período futuro. Dessa forma, podemos aproveitar melhor os objetos e, assim, diminuir o custo com os objetos necessários para cortar todos os itens das ordens de trabalho no horizonte de planejamento.

Seja $\bar{m}_{s}$ o número de objetos disponíveis no período $s$ (isto é, o número $m_{s}$ de objetos de entrada mais o número de sobras dos períodos anteriores que não foram utilizadas), para $s=p+1, \ldots, P$. Considerando que cada objeto pode gerar, no máximo, duas sobras aproveitáveis, temos que

$$
\bar{m}_{s}=m_{s}+2\left(\sum_{i=1}^{\min (s-p, \xi)} 2^{i-1} m_{s-i}\right), \quad s=p, \ldots, P
$$

onde $\xi \leq P-p$ indica o número de períodos que uma sobra está disponível para utilização. Dessa forma, se $\xi=1$, apenas as sobras geradas a partir de objetos de entrada do período $s-1$ estão disponíveis no período $s$, e se $\xi=P-p$, as sobras de qualquer período anterior estão disponíveis no período $s$. Para modelar o nosso problema, os $m_{s}$ primeiros objetos de $\bar{m}_{s}$ referem-se aos objetos de entrada do período $s$, os dois próximos objetos referem-se, respectivamente, a sobra da direita e a sobra superior do objeto $j=1$ do período $s-1$, os dois seguintes referem-se às sobras do objeto $j=2$ do período $s-1$, e assim por diante. A Figura 2.5 ilustra esse esquema de posicionamento para uma instância com três períodos e $\operatorname{com} \xi=2$. Na figura, temos que $m_{1}=m_{2}=m_{3}=1$ e, assim, $\bar{m}_{1}=m_{1}=1, \bar{m}_{2}=m_{2}+2 \times m_{1}=3$ e $\bar{m}_{3}=m_{3}+2 \times m_{2}+4 \times m_{1}=7$. Por outro lado, se considerarmos $\xi=1$, as sobras ficam disponíveis para utilização por apenas um período, ou seja, apenas as sobras geradas de objetos de entrada do período $s-1$ estão disponíveis no período $s$. Dessa forma, supondo $\xi=1$ na instância da Figura 2.5, apenas as sobras $O_{22}, O_{23}, O_{32}$ e $O_{33}$ ficam disponíveis, como mostra da Figura 2.6.

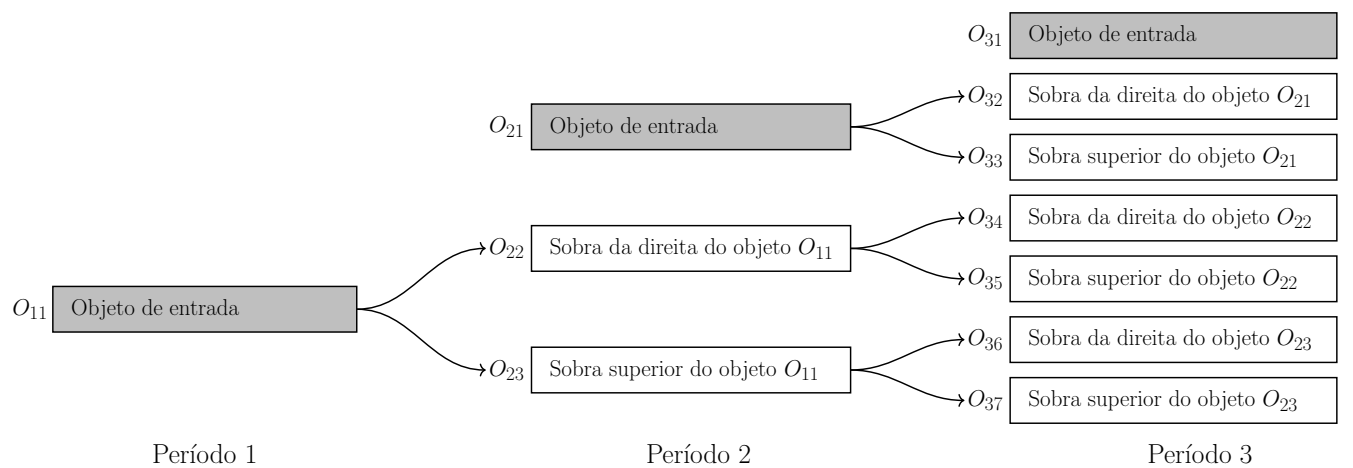

Figura 2.5: Posicionamento, usado pelo modelo do problema proposto, dos objetos de entrada e dos objetos originados de sobras para uma instância com três períodos, com um objeto de entrada (em cinza) em cada período e considerando $\xi=2$.

Perceba que o número de objetos disponíveis em cada período $\left(\bar{m}_{s}\right)$ cresce exponencialmente quando $\xi=P-p$. Para contornarmos este inconveniente, uma primeira alternativa é limitar o número de períodos que uma sobra gerada a partir de um objeto de entrada do período $s$ fica 
disponível, ou seja, fazendo $\xi<P-p$ (como ilustrado na Figura 2.6). No entanto, essa estratégia pode diminuir a qualidade das soluções, uma vez que algumas sobras aproveitáveis e não utilizadas podem ser descartadas. Outra alternativa é considerar que cada objeto só gera uma sobra guilhotinada (ou a da direita ou a superior) ao invés de duas ou uma sobra não-guilhotinada (que pode estar em qualquer parte do objeto). Essa alternativa, entretanto, pode obter soluções de pior qualidade e, por isso, optamos por considerar que cada objeto utilizado pode gerar duas sobras guilhotinadas, sabendo que isso limita o número de períodos que conseguimos tratar.

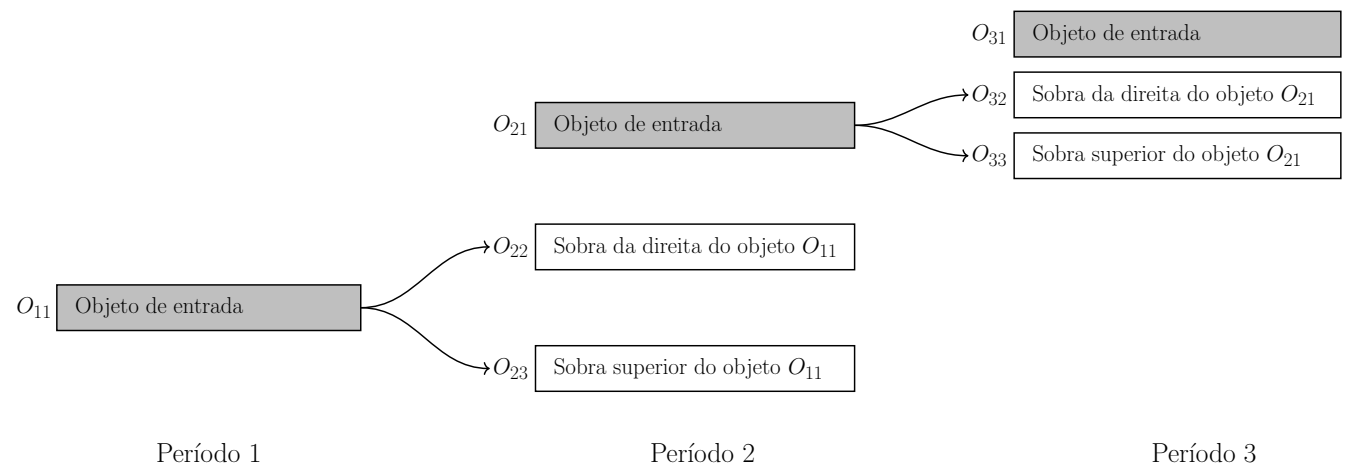

Figura 2.6: Objetos de entrada (em cinza) e objetos originados de sobras para uma instância com três períodos e supondo $\xi=1$. Nesse caso, apenas as sobras geradas de objetos de entrada do período $s$ ficam disponíveis no período $s+1$.

Seja $u_{s j} \in\{0,1\}, s=p, \ldots, P, j=1, \ldots, \bar{m}_{s}$, indicando se o objeto $j$ do período $s$ é usado para cortar algum item da ordem de trabalho ou se o objeto, originado de uma sobra, é selecionado para ser uma sobra aproveitável remanescente no último período. Neste caso, temos que $u_{s j}=1$, caso contrário, $u_{s j}=0$. Seja $v_{s i j} \in\{0,1\}, s=p, \ldots, P, i=1, \ldots, n_{s}, j=1, \ldots, \bar{m}_{s}$, para atribuir o item $i$ ao objeto $j$ no período $s\left(v_{s i j}=1\right)$ ou não $\left(v_{s i j}=0\right)$. Assim, devemos ter que

$$
\begin{array}{ll}
v_{s i j} \leq u_{s j}, & s=p, \ldots, P, i=1, \ldots, n_{s}, j=1, \ldots, \bar{m}_{s}, \\
\sum_{j=1}^{\bar{m}_{s}} v_{s i j}=1, & s=p, \ldots, P, i=1, \ldots, n_{s} .
\end{array}
$$

As restrições (2.19) garantem que um item $i$ do período $s$ não seja atribuído a um objeto $j$ no período $s\left(v_{s i j}=1\right)$ caso o objeto não seja usado $\left(u_{s j}=0\right)$. Já o conjunto de restrições $(2.20)$ impõe que cada item seja atribuído a exatamente um objeto. Dessa forma, podemos definir a função objetivo que minimiza o custo dos objetos utilizados como

$$
\sum_{s=p}^{P} \sum_{j=1}^{m_{s}} c_{s j} W_{s j} H_{s j} u_{s j} .
$$

O próximo passo é definir as dimensões dos objetos de cada período $s>1$ originados de sobras dos objetos de algum período anterior a $s$. Usando as mesmas definições apresentadas na seção anterior, sejam $t_{s j}$ e $r_{s j}$ as variáveis associadas, respectivamente, à altura da sobra superior e a largura do lado direito do objeto $j$ no período $s$, para $s=p, \ldots, P, j=1, \ldots, \bar{m}_{s}$. Dessa forma, as dimensões das 
sobras de cada objeto $j$ no período $s$ são dadas de acordo com o tipo de corte do objeto (Caso A ou Caso B), como ilustrado na Figura 2.7. Como queremos considerar que as sobras aproveitáveis de um período são objetos disponíveis no período seguinte, devemos definir a altura e a largura desses objetos como as dimensões das sobras aproveitáveis do período anterior. Portanto, as dimensões das sobras do objeto $j$ do período $s, s=p, \ldots, P, j=1, \ldots, \bar{m}_{s}$, serão as dimensões dos novos objetos no período $s+1$ ou sobras remanescentes no final do horizonte considerado. A Figura 2.8 ilustra todos os objetos disponíveis para uma instância com dois períodos ( $\operatorname{com} p=1$ e $P=2)$ e $\xi=P-p$. No primeiro período, temos dois objetos dados como entrada (em cinza). Já no segundo, além do objeto dado como entrada, também temos os outros quatro objetos originados das possíveis sobras, com as dimensões baseadas no tipo de corte dos dois objetos do período anterior. Caso a instância tivesse um terceiro período, este teria os seguintes objetos: os objetos dados como entrada para o período 3 (armazenado em $m_{3}$ ) e as duas sobras de cada objeto do segundo período, já que $\xi=P-p$. Assim, $\bar{m}_{3}=m_{3}+10$.

Sejam $\bar{W}_{s j}$ e $\bar{H}_{s j}$ variáveis artificiais contínuas associadas, respectivamente, a largura e a altura do objeto $j$ no período $s$, para $s=p, \ldots, P, j=1, \ldots, \bar{m}_{s}$, com $\bar{W}_{s j}$ e $\bar{H}_{s j}$ exatamente iguais a $W_{s j}$ e $H_{s j}$, respectivamente, caso o objeto $j$ no período $s$ seja usado $\left(u_{s j}=1\right)$ ou nulas caso contrário, para $s=p, \ldots, P, j=1, \ldots, m_{s}$. Assim,

$$
\begin{aligned}
& \bar{W}_{s j}=W_{s j} u_{s j}, \quad s=p, \ldots, P, j=1, \ldots, m_{s}, \\
& \bar{H}_{s j}=H_{s j} u_{s j}, \quad s=p, \ldots, P, j=1, \ldots, m_{s} .
\end{aligned}
$$

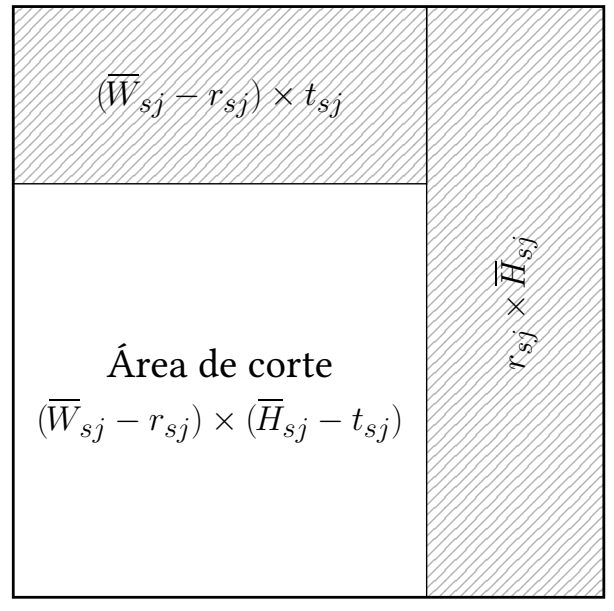

(a) Caso A

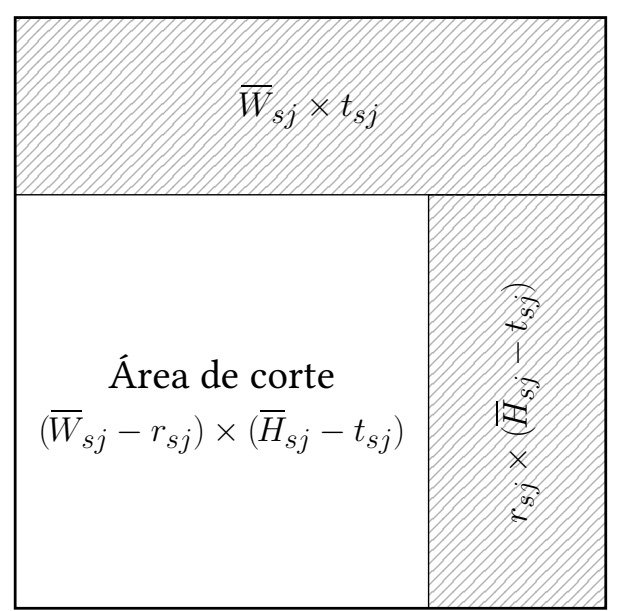

(b) Caso B

Figura 2.7: Dimensões da área de corte e das sobras do objeto $j$ no período $s$, para $s=p, \ldots, P, j=$ $1, \ldots, \bar{m}_{s}$, para os dois casos de cortes possíveis. Baseada em Andrade et al. [6]. 


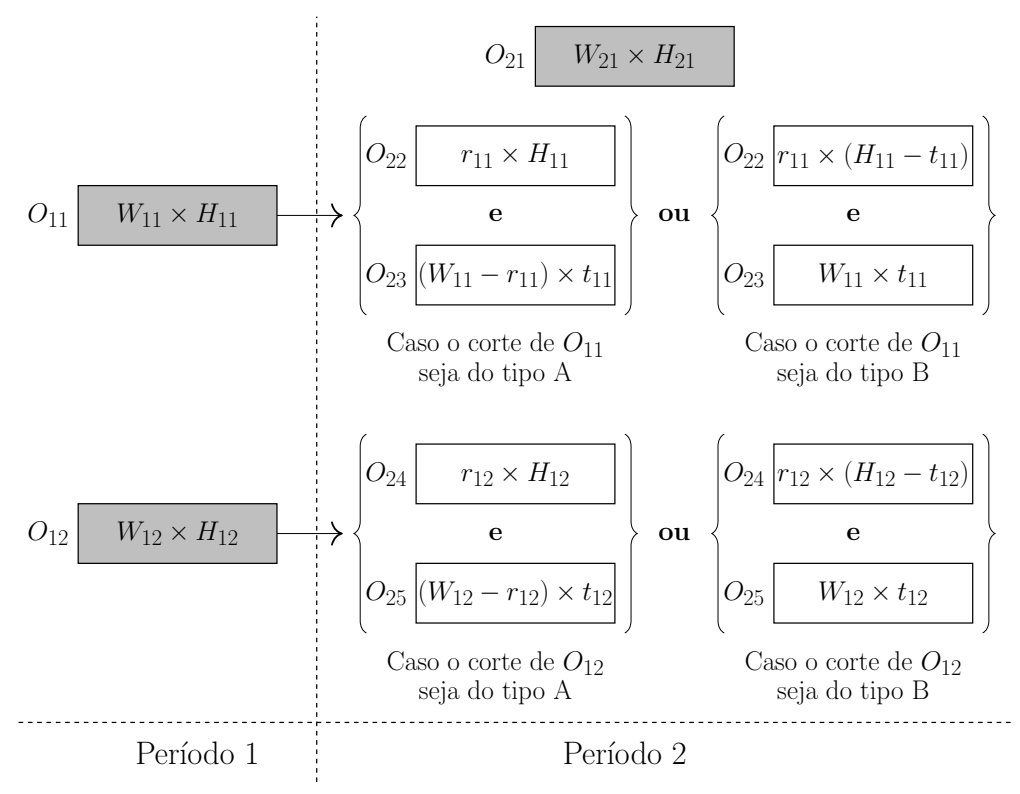

Figura 2.8: Dimensões dos objetos disponíveis para uma instância com dois períodos e três objetos de entrada (em cinza), dois no primeiro e um no segundo período. Supondo $\xi=P-p$, no segundo período, as sobras aproveitáveis dos objetos do período anterior são consideradas objetos, com dimensões baseadas no tipo de corte guilhotinado (Caso A ou Caso B).

Como dito anteriormente, se um objeto $j$ do período $s$ é de fato uma sobra aproveitável de um período anterior, então suas dimensões devem ser as mesmas da sobra, para $s=p+1, \ldots, P, j=$ $m_{s}+1, \ldots, \bar{m}_{s}$. Assim, os valores de $\bar{W}_{s j}$ e $\bar{H}_{s j}, s=p+1, \ldots, P, j=m_{s}+1, \ldots, \bar{m}_{s}$, devem coincidir, respectivamente, com a largura e a altura da sobra do objeto do período anterior. Como definimos dois padrões de corte, $\bar{W}_{s j}$ e $\bar{H}_{s j}$ devem ter seus valores baseados no tipo de corte que gerou a sobra. Assim, como na seção anterior, sejam as variáveis binárias $\eta_{s j} \in\{0,1\}$ associadas ao tipo de corte guilhotinado (Caso A ou Caso B) do objeto, para $s=p, \ldots, P, j=1, \ldots, \bar{m}_{s}$. Para definirmos a largura e a altura do objeto do período $s>p$ originado da sobra direita do objeto $j$ do período $s-1$, de acordo com o tipo de corte, podemos escrever as restrições:

$$
\begin{array}{cclll}
\eta_{s-1, j}=0 & \Longrightarrow \bar{W}_{s, m_{s}+2 j-1}=r_{s-1, j} & \text { e } & \bar{H}_{s, m_{s}+2 j-1}=\bar{H}_{s-1, j} & \text { (Caso A) } \\
\eta_{s-1, j}=1 \Longrightarrow \bar{W}_{s, m_{s}+2 j-1}=r_{s-1, j} & \text { e } & \bar{H}_{s, m_{s}+2 j-1}=\bar{H}_{s-1, j}-t_{s-1, j}, \quad \text { (Caso B) } \\
& & s=p+1, \ldots, P, j=1, \ldots, \frac{\bar{m}_{s}-m_{s}}{2} .
\end{array}
$$

e para os objetos do período $s>p$ associados às sobras superiores podemos escrever:

$$
\begin{aligned}
& \eta_{s-1, j}=0 \Longrightarrow \bar{W}_{s, m_{s}+2 j}=\bar{W}_{s-1, j}-r_{s-1, j} \quad \text { e } \bar{H}_{s, m_{s}+2 j}=t_{s-1, j}, \quad \text { (Caso A) } \\
& \eta_{s-1, j}=1 \Longrightarrow \bar{W}_{s, m_{s}+2 j}=\bar{W}_{s-1, j} \quad \text { e } \bar{H}_{s, m_{s}+2 j}=t_{s-1, j}, \quad \text { (Caso B) } \\
& \quad s=p+1, \ldots, P, j=1, \ldots, \frac{\bar{m}_{s}-m_{s}}{2} .
\end{aligned}
$$

Essas restrições podem ser representadas usando a reformulação big- $M$, respectivamente, da seguinte maneira: 


$$
\begin{aligned}
0 \leq \bar{W}_{s, m_{s}+2 j-1} & \leq r_{s-1, j} \\
0 \leq \bar{H}_{s, m_{s}+2 j-1} \leq & \bar{H}_{s-1, j}+\hat{H} \eta_{s-1, j} \\
0 \leq \bar{H}_{s, m_{s}+2 j-1} \leq & \bar{H}_{s-1, j}-t_{s-1, j}+\hat{H}\left(1-\eta_{s-1, j}\right) \\
& \quad s=p+1, \ldots, P, j=1, \ldots, \frac{\bar{m}_{s}-m_{s}}{2},
\end{aligned}
$$

$\mathrm{e}$

$$
\begin{aligned}
& 0 \leq \bar{W}_{s, m_{s}+2 j} \leq \bar{W}_{s-1, j}-r_{s-1, j}+\hat{W} \eta_{s-1, j} \\
& 0 \leq \bar{W}_{s, m_{s}+2 j} \leq \bar{W}_{s-1, j}+\hat{W}\left(1-\eta_{s-1, j}\right) \\
& 0 \leq \bar{H}_{s, m_{s}+2 j} \leq t_{s-1, j} \\
& \quad s=p+1, \ldots, P, j=1, \ldots, \frac{\bar{m}_{s}-m_{s}}{2} .
\end{aligned}
$$

O conjunto de restrições (2.23) define as dimensões dos novos objetos referentes às sobras da direita do período anterior. Observe que a largura do novo objeto, independentemente do tipo de corte, sempre está relacionada apenas a largura da sobra $\left(r_{s-1, j}\right)$ do objeto do período anterior. Por outro lado, a altura depende do tipo de corte, que pode ser $\bar{H}_{s-1, j}$ (Caso A) ou $\bar{H}_{s-1, j}-t_{s-1, j}$ (Caso B). Para os objetos referentes às sobras da parte superior, as restrições (2.24) garantem que a largura dos novos objetos é $\bar{W}_{s-1, j}-r_{s-1, j}$ (Caso A) ou $\bar{W}_{s-1, j}$ (Caso B) e as alturas desses objetos sempre estão associadas às alturas das sobras $\left(t_{s-1, j}\right)$.

Definido a largura e a altura de cada objeto proveniente de uma sobra aproveitável, agora devemos garantir que as dimensões das sobras desses objetos sejam menores que as dimensões do próprio objeto ao qual elas estão associadas, sempre que o objeto for utilizado no período e sejam nulas, caso contrário. Isso pode ser modelado da seguinte forma

$$
\begin{aligned}
0 & \leq r_{s j} \leq W_{s j} u_{s j} \\
0 & \leq t_{s j} \leq H_{s j} u_{s j} \\
0 & \leq r_{s j} \leq \bar{W}_{s j} u_{s j} \\
0 & \leq t_{s j} \leq \bar{H}_{s j} u_{s j}
\end{aligned}
$$

O conjunto de restrições (2.25) garante que os valores de $r_{s j}$ e $t_{s j}$, que determinam o tamanho das sobras do objeto $j$ do período $s$, sejam menores que as dimensões do objeto, caso o objeto seja utilizado $\left(u_{s j}=1\right)$, e nulas, caso contrário $\left(u_{s j}=0\right)$. As restrições (2.26) garantem a mesma coisa com relação a objetos provenientes de sobras aproveitáveis de períodos anteriores. No entanto, as restrições (2.26) são não-lineares, pois incluem o produto entre as variáveis de decisão $\bar{W}_{s j}$ e $u_{s j}$, e entre $\bar{H}_{s j}$ e $u_{s j}$. Para contornar este inconveniente, podemos substituir as restrições (2.26) pelas restrições

$$
\begin{aligned}
0 & \leq r_{s j} \leq \bar{W}_{s j} \\
0 & \leq t_{s j} \leq \bar{H}_{s j} \\
0 & \leq r_{s j} \leq \hat{W} u_{s j} \\
0 & \leq t_{s j} \leq \hat{H} u_{s j}
\end{aligned}
$$


onde $\hat{W}=\max _{s=p, \ldots, P, j=1, \ldots, m_{s}}\left\{W_{s j}\right\}$ e $\hat{H}=\max _{s=p, \ldots, P, j=1, \ldots, m_{s}}\left\{H_{s j}\right\}$. As restrições (2.28) garantem que sempre que o objeto não for usado $\left(u_{s j}=0\right)$ as variáveis $r_{s j}$ e $t_{s j}$ serão nulas. Caso contrário, $r_{s j}$ e $t_{s j}$ podem assumir qualquer valor positivo menor ou igual a $\bar{W}_{s j}$ e $\bar{H}_{s j}$, respectivamente, o que é assegurado pelas restrições (2.27).

Perceba que, se um objeto $j$ de um período $s$ não for utilizado $\left(u_{s j}=0\right)$, as restrições $(2.23) \mathrm{e}$ (2.24) em conjunto com as restrições (2.27) e (2.28) fazem com que as sobras desse objeto tenham dimensões nulas e, assim, no período $s+1$ as dimensões dos dois objetos associados as duas sobras do objeto $j$ do período $s$ também terão dimensões nulas. Entretanto, isso só deve acontecer para os objetos que não foram originados de alguma sobra em algum período anterior (objetos de entrada), enquanto que os objetos originados de sobras, quando não utilizados no período $s$, ou seja, quando não tiverem itens atribuídos, devem estar disponíveis no período $s+1 \mathrm{com}$ as mesmas dimensões do período $s$, como podemos observar nas soluções da Figura 2.4. Neste caso, no período $s+1$, um dos dois objetos associados as sobras do objeto $j$ do período $s$ deve ter as mesmas dimensões do objeto $j$ do período $s$ e o outro deve ter dimensões nulas, já que o objeto $j$ não pode ser cortado para produzir as duas possíveis sobras se não estiver sendo utilizado. Sem perda de generalidade, definimos que sempre que um objeto $j$ originado de alguma sobra não for utilizado no período $s$, no próximo período, o objeto originado da sobra da direita do objeto $j$ terá as mesmas dimensões deste objeto, e o objeto referente a sobra da parte superior do objeto $j$ no período $s+1$ terá dimensões nulas. Dessa forma, todos os objetos originados de alguma sobra que não forem utilizados no período $s$, estarão disponíveis no período $s+1$. Para garantir isso, devemos ter:

$$
\begin{aligned}
& 0 \leq \bar{W}_{s, m_{s}+2 j-1} \leq r_{s-1, j}+\hat{W}\left(1-u_{s-1, j}\right) \\
& 0 \leq \bar{W}_{s, m_{s}+2 j-1} \leq \bar{W}_{s-1, j}+\hat{W} u_{s-1, j} \\
& 0 \leq \bar{H}_{s, m_{s}+2 j-1} \leq \bar{H}_{s-1, j}+\hat{H} \eta_{s-1, j}+\hat{H}\left(1-u_{s-1, j}\right) \\
& 0 \leq \bar{H}_{s, m_{s}+2 j-1} \leq \bar{H}_{s-1, j}-t_{s-1, j}+\hat{H}\left(1-\eta_{s-1, j}\right)+\hat{H}\left(1-u_{s-1, j}\right) \\
& 0 \leq \bar{H}_{s, m_{s}+2 j-1} \leq \bar{H}_{s-1, j}+\hat{H} u_{s-1, j} \quad s=p+1, \ldots, P, j=1, \ldots, \frac{\bar{m}_{s}-m_{s}}{2}, \\
& 0 \leq \bar{W}_{s, m_{s}+2 j} \leq \bar{W}_{s-1, j}-r_{s-1, j}+\hat{W} \eta_{s-1, j}+\hat{W}\left(1-u_{s-1, j}\right) \\
& 0 \leq \bar{W}_{s, m_{s}+2 j} \leq \bar{W}_{s-1, j}+\hat{W}\left(1-\eta_{s-1, j}\right)+\hat{W}\left(1-u_{s-1, j}\right) \\
& 0 \leq \bar{W}_{s, m_{s}+2 j} \leq \hat{W}_{s-1, j} \\
& 0 \leq \bar{H}_{s, m_{s}+2 j} \leq t_{s-1, j}+\hat{H}\left(1-u_{s-1, j}\right) \\
& 0 \leq \bar{H}_{s, m_{s}+2 j} \leq \hat{H} u_{s-1, j} \\
& 0 \\
& \quad s=p+1, \ldots, P-1, j=m_{s}+1, \ldots, \bar{m}_{s} .
\end{aligned}
$$

As restrições (2.29), juntamente com as restrições (2.31), definem as dimensões do objeto $m_{s}+2 j-$ 1 no período $s$, que é originado da sobra do lado direito do objeto $j$ do período $s-1$, de acordo com o tipo de corte. Nessas restrições, o último " $M$-termo" garante que se o objeto do período anterior, 
ao qual o novo objeto está associado, não tiver sido utilizado $\left(u_{s-1, j}=0\right)$, então sua altura e largura poderão ser, no máximo, $\bar{W}_{s-1, j}$ e $\bar{H}_{s-1, j}$, respectivamente. Caso contrário, o objeto $m_{s}+2 j-1$ do período $s$ terá, no máximo, a altura e a largura da sobra do objeto $j$ do período $s-1$, dependendo do tipo de corte. Já as restrições (2.30), em conjunto com as restrições (2.31), definem as dimensões do objeto $m_{s}+2 j$ no período $s$ associado à sobra da parte superior do objeto $j$ do período $s-1$. Essas dimensões devem ser nulas caso o objeto $j$, ao qual o objeto $m_{s}+2 j$ está associado, não tenha sido utilizado $\left(u_{s-1, j}=0\right)$, uma vez que as dimensões originais do objeto $m_{s}+2 j$ do período anterior foram usadas como sendo as dimensões das sobras da direita nas restrições (2.29), o que é garantido pelo último " $M$-termo" dessas restrições. Caso contrário, as dimensões do objeto $m_{s}+2 j$ terão, no máximo, as dimensões das sobras da parte superior, de acordo com o tipo de corte, do objeto $j$ do período anterior. Já que as variáveis $\bar{W}_{s, m_{s}+2 j-1}, \bar{H}_{s, m_{s}+2 j-1}, \bar{W}_{s, m_{s}+2 j}$ e $\bar{H}_{s, m_{s}+2 j}$ estão associadas às áreas dos objetos e/ou de possíveis sobras e queremos minimizar o custo dos objetos utilizados e, entre as soluções de custo mínimo, escolher aquela que maximiza o valor das sobras finais, em uma solução, essas variáveis atingirão seus valores máximos possíveis. As restrições (2.31) garantem que um objeto originado de sobra só será selecionado $\left(u_{s j}=1\right)$ se algum item for atribuído ao objeto $\left(\sum_{i=1}^{n_{s}} v_{s i j} \geq 1\right)$.

A Figura 2.9 ilustra as dimensões dos objetos para uma solução de uma instância com três períodos e, inicialmente, com um objeto de entrada (retângulos em cinza) no primeiro e terceiro período e com dois no segundo período. Para essa instância, supomos que $\xi=2$, isto é, as sobras geradas no período $s$ podem ficar disponíveis até o período $s+2$. Os objetos comprados (utilizados) possuem, à direita, o tipo de corte (Caso A ou Caso B) usado. No primeiro período, o objeto disponível é cortado usando o tipo de corte do Caso B, gerando duas sobras para o próximo período. Essas sobras possuem dimensões baseadas no tipo de corte que o objeto teve. No período 2, temos quatro objetos disponíveis, dois de entrada e dois referentes às sobras do objeto do período anterior. Nesse período, o objeto de entrada $\mathrm{O}_{22}$ é comprado e cortado usando o tipo de corte do Caso B e o objeto $O_{23}$ é cortado usando o tipo de corte do Caso A. Como o primeiro objeto do segundo período é um objeto de entrada e não foi comprado, as variáveis $r_{21}$ e $t_{21}$ são nulas, assim os objetos $O_{32}$ e $O_{33}$ não podem conter nenhum item, já que uma de suas dimensões vale zero. O objeto $O_{24}$ também não é utilizado, porém, por ser originado de uma sobra, ele deve estar disponível no próximo período com as mesmas dimensões que possui no período 2. Nesse caso, o objeto $O_{38}$, referente à sobra da direita, possui as dimensões do objeto $O_{24}$, enquanto o objeto $O_{39}$, referente à sobra superior, têm dimensões nulas. Note que o objeto $\mathrm{O}_{23}$, mesmo sendo originado de uma sobra, também gerou sobras que são utilizadas no período 3.

A Figura 2.10 ilustra a mesma instância da Figura 2.9, mas agora consideramos $\xi=1$, ou seja, no período $s>1$, só existem sobras que foram geradas a partir de objetos de entrada do período $s-1$. Assim, no terceiro período, as sobras associadas ao objeto $O_{11}$ deixam de existir. Diminuindo o número de objetos disponíveis em cada período, também reduzimos o número de variáveis do modelo, o que pode fazer com que um software de otimização de propósito geral consiga resolver problemas de tamanhos maiores. 


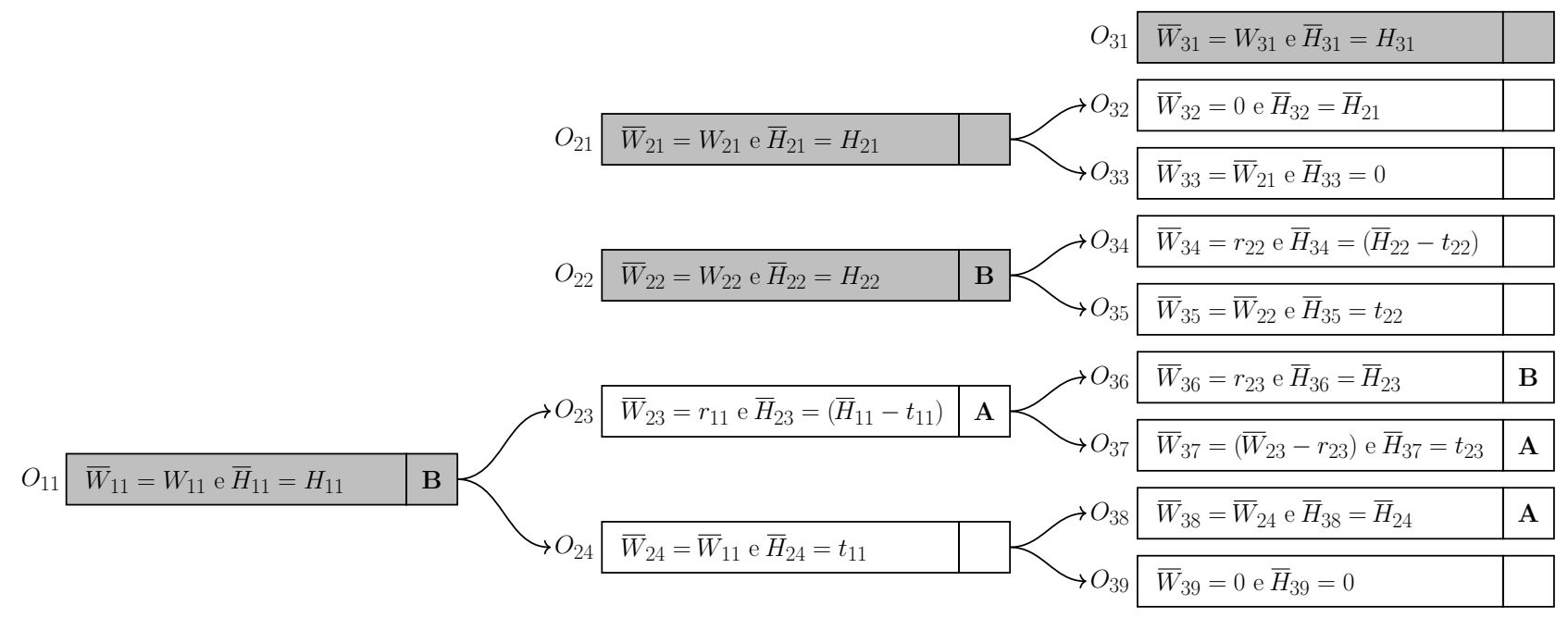

Período 1

Período 2

Período 3

Figura 2.9: Dimensões dos objetos de uma instância hipotética com três períodos e com $\xi=2$. Cada retângulo representa um objeto do período com suas dimensões, sendo os retângulos em cinza associados aos objetos de entrada. Caso o objeto seja usado, à direita, temos o tipo de corte (Caso A ou Caso B) utilizado.

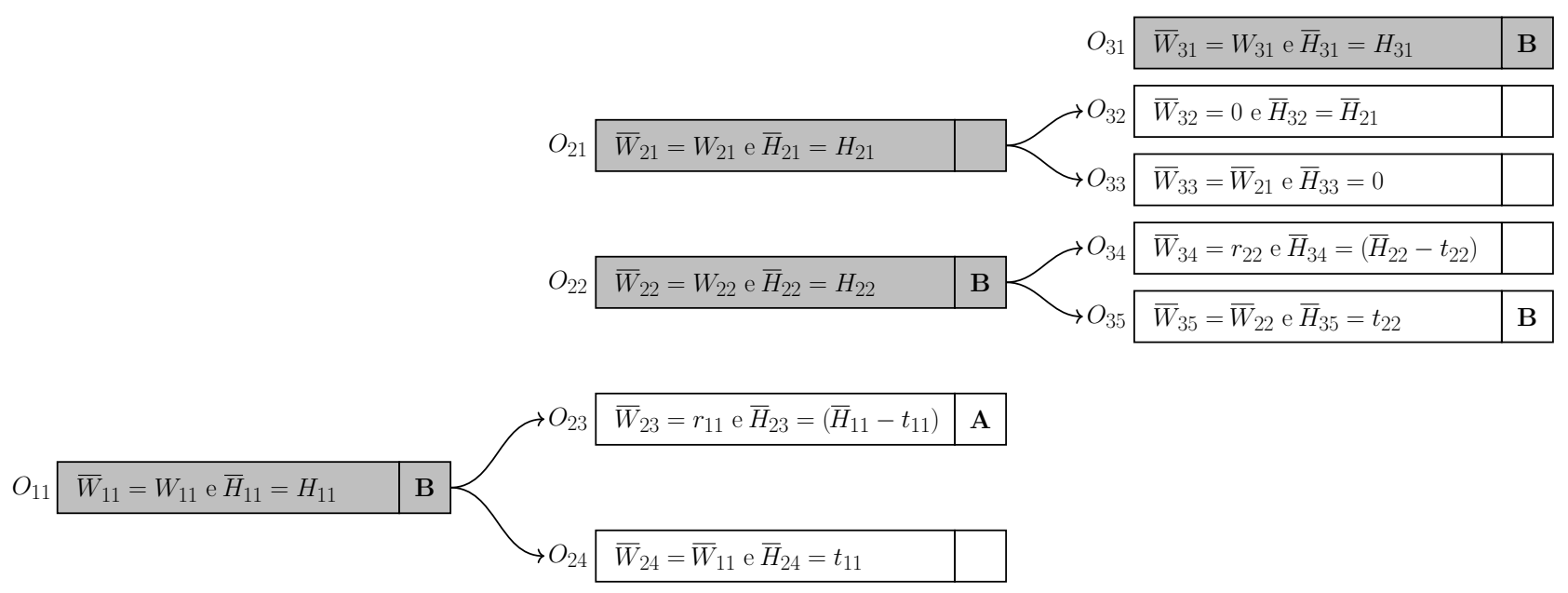

Período 1

Período 2

Período 3

Figura 2.10: Dimensões dos objetos de uma instância hipotética com três períodos e com $\xi=1$. Retângulos em cinza representam objetos de entrada. Note que agora, diferente do que acontece no exemplo Figura 2.9, as sobras geradas pelos objetos de entrada do período $s$ só podem ser utilizadas no período $s+1$ e depois são descartadas.

Definidas as dimensões dos objetos de cada período, agora devemos modelar o posicionamento dos itens dentro dos objetos, evitando sobreposições. Como apresentado na seção anterior, seja $\left(x_{s i}, y_{s i}\right), s=p, \ldots, P, i=1, \ldots, n_{s}$, a coordenada do centro Cartesiano do item $i$ do período $s$. Podemos escrever restrições que garantem que o item $i$ deve estar contido dentro da "Área de corte" (veja Figura 2.7) do objeto $j$ no período $s$ sempre que $v_{s i j}=1$ da seguinte forma: 


$$
\begin{array}{lll}
x_{s i}-w_{s i} / 2 \geq 0, & s=p, \ldots, P, i=1, \ldots, n_{s}, \\
y_{s i}-h_{s i} / 2 \geq 0, & s=p, \ldots, P, i=1, \ldots, n_{s}, \\
x_{s i}+w_{s i} / 2 \leq \bar{W}_{s j}-r_{s j}+\hat{W}\left(1-v_{s i j}\right), & s=p, \ldots, P, i=1, \ldots, n_{s}, j=1, \ldots, \bar{m}_{s}, \\
y_{s i}+h_{s i} / 2 \leq \bar{H}_{s j}-t_{s j}+\hat{H}\left(1-v_{s i j}\right), & s=p, \ldots, P, i=1, \ldots, n_{s}, j=1, \ldots, \bar{m}_{s} .
\end{array}
$$

Para evitar sobreposição entre o item $i$ e $i^{\prime}$, que são atribuídos ao objeto $j$ no período $s$, podemos escrever as restrições:

$$
\begin{array}{r}
v_{s i j}=v_{s i^{\prime} j}=1 \Longrightarrow\left|x_{s i}-x_{s i^{\prime}}\right| \geq\left(w_{s i}+w_{s i^{\prime}}\right) / 2 \text { ou }\left|y_{s i}-y_{s i^{\prime}}\right| \geq\left(h_{s i}+h_{s i^{\prime}}\right) / 2, \\
s=p, \ldots, P, i=1, \ldots, n_{s}, i^{\prime}=i+1, \ldots, n_{s}, j=1, \ldots, \bar{m}_{s} .
\end{array}
$$

Reescrevendo " $|a| \geq b$ " como " $a \geq b$ ou $a \leq-b$ " e definindo as variáveis auxiliares $\pi_{\text {sii' }}, \tau_{\text {sii' }} \in$ $\{0,1\}, s=p, \ldots, P, i=1, \ldots, n_{s}, i^{\prime}=i+1, \ldots, n_{s}$, para a reformulação big- $M$ dos quatro termos das disjunções, obtermos as seguintes restrições:

$$
\begin{aligned}
x_{s i^{\prime}}+w_{s i^{\prime}} / 2 & \leq x_{s i}-w_{s i} / 2+\hat{W}\left(1-v_{s i j}\right)+\hat{W}\left(1-v_{s i^{\prime} j}\right)+\hat{W} \pi_{s i i^{\prime}}+\hat{W} \tau_{s i i^{\prime}}, \\
x_{s i^{\prime}}-w_{s i^{\prime}} / 2 \geq & x_{s i}+w_{s i} / 2-\hat{W}\left(1-v_{s i j}\right)-\hat{W}\left(1-v_{s i^{\prime} j}\right)-\hat{W} \pi_{s i i^{\prime}}-\hat{W}\left(1-\tau_{s i i^{\prime}}\right), \\
y_{s i^{\prime}}+h_{s i^{\prime}} / 2 \leq & y_{s i}-h_{s i} / 2+\hat{H}\left(1-v_{s i j}\right)+\hat{H}\left(1-v_{s i^{\prime} j}\right)+\hat{H}\left(1-\pi_{s i i^{\prime}}\right)+\hat{H} \tau_{s i i^{\prime}}, \\
y_{s i^{\prime}}-h_{s i^{\prime}} / 2 \geq & y_{s i}+h_{s i} / 2-\hat{H}\left(1-v_{s i j}\right)-\hat{H}\left(1-v_{s i^{\prime} j}\right)-\hat{H}\left(1-\pi_{s i i^{\prime}}\right)-\hat{H}\left(1-\tau_{s i i^{\prime}}\right), \\
& s=p, \ldots, P, i=1, \ldots, n_{s}, i^{\prime}=i+1, \ldots, n_{s}, j=1, \ldots, \bar{m}_{s} .
\end{aligned}
$$

Como na Seção 2.1, perceba que a troca da posição de dois itens $i$ e $i^{\prime}$ idênticos no período $s$ $\left(w_{s i}=w_{s i^{\prime}}\right.$ e $\left.h_{s i}=h_{s i^{\prime}}\right)$ gera uma solução simétrica. Para evitarmos essa situação podemos adotar a estratégia proposta por [5] para diminuir o número de soluções simétricas. Suponha que os itens idênticos estão numerados consecutivamente, que existem $\tilde{n}_{s}$ tipos diferentes de itens no período $s$ e que há $z_{s q}$ itens do $q$-ésimo tipo, $s=p, \ldots, P, q=1, \ldots, \tilde{n}_{s}$, com $\sum_{q=1}^{\tilde{n}_{s}} z_{s q}=n_{s}$. Denotando $o_{s q}=\sum_{q^{\prime}=1}^{q-1} z_{s q^{\prime}}$, temos que os itens do $q$-ésimo tipo estão numerados de $o_{s q}+1$ a $o_{s q}+z_{s q}$. Dessa forma, as restrições de não sobreposição podem ser reescritas da seguinte forma:

$$
\begin{gathered}
x_{s i^{\prime}}-w_{s i^{\prime}} / 2 \geq x_{s i}+w_{s i} / 2-\hat{W}\left(1-v_{s i j}\right)-\hat{W}\left(1-v_{s i^{\prime} j}\right)-\hat{W} \pi_{s i i^{\prime}}, \\
y_{s i^{\prime}}-h_{s i^{\prime}} / 2 \geq y_{s i}+h_{s i} / 2-\hat{H}\left(1-v_{s i j}\right)-\hat{H}\left(1-v_{s i^{\prime} j}\right)-\hat{H}\left(1-\pi_{s i i^{\prime}}\right), \\
s=p, \ldots, P, q=1, \ldots, \tilde{n}_{s}, i=o_{s q}+1, \ldots, o_{s q}+z_{s q}, i^{\prime}=i+1, \ldots, o_{s q}+z_{s q}, j=1, \ldots, \bar{m}_{s},
\end{gathered}
$$

$\mathrm{e}$

$$
\begin{aligned}
& x_{s i^{\prime}}+w_{s i^{\prime}} / 2 \leq x_{s i}-w_{s i} / 2+\hat{W}\left(1-v_{s i j}\right)+\hat{W}\left(1-v_{s i^{\prime} j}\right)+\hat{W} \pi_{s i i^{\prime}}+\hat{W} \tau_{s i i^{\prime}}, \\
& x_{s i^{\prime}}-w_{s i^{\prime}} / 2 \geq x_{s i}+w_{s i} / 2-\hat{W}\left(1-v_{s i j}\right)-\hat{W}\left(1-v_{s i^{\prime} j}\right)-\hat{W} \pi_{s i i^{\prime}}-\hat{W}\left(1-\tau_{s i i^{\prime}}\right), \\
& y_{s i^{\prime}}+h_{s i^{\prime}} / 2 \leq y_{s i}-h_{s i} / 2+\hat{H}\left(1-v_{s i j}\right)+\hat{H}\left(1-v_{s i^{\prime} j}\right)+\hat{H}\left(1-\pi_{s i i^{\prime}}\right)+\hat{H} \tau_{s i i^{\prime}}, \\
& y_{s i^{\prime}}-h_{s i^{\prime}} / 2 \geq y_{s i}+h_{s i} / 2-\hat{H}\left(1-v_{s i j}\right)-\hat{H}\left(1-v_{s i^{\prime} j}\right)-\hat{H}\left(1-\pi_{s i i^{\prime}}\right)-\hat{H}\left(1-\tau_{s i i^{\prime}}\right), \\
& s=p, \ldots, P, q=1, \ldots, \tilde{n}_{s}, i=o_{s q}+1, \ldots, o_{s q}+z_{s q}, i^{\prime}=o_{s q}+z_{s q}+1, \ldots, n_{s}, j=1, \ldots, \bar{m}_{s} .
\end{aligned}
$$


O conjunto de restrições (2.33) modela a não sobreposição entre itens idênticos usando um número bem menor de restrições e variáveis binárias, com o recurso adicional de evitar o tipo de soluções simétricas. Já o conjunto de restrições (2.34) modela a não sobreposição entre pares de itens diferentes. Nesse conjunto de restrição, os dois primeiros " $M$-termos" das restrições anulam o efeito das restrições sempre que $v_{s i j}=0$ ou $v_{s i}{ }^{\prime} j=0$, o que significa que o item $i$ ou o item $i^{\prime}$ do período $s$ não foram atribuídos ao objeto $j$ e, portanto, não há sobreposição entre eles no objeto $j$. Os outros dois " $M$-termos" das restrições, junto com as quatro possíveis combinações dos valores das variáveis binárias $\pi_{s i i^{\prime}}$ e $\tau_{s i i^{\prime}}$, modelam a disjunção que garante que, no período $s$, o item $i^{\prime}$ deve estar "à esquerda", "à direita", "acima” ou "abaixo" do item $i$, sempre que os itens $i$ e $i^{\prime}$ forem atribuídos ao mesmo objeto no mesmo período, evitando sobreposição entre estes itens.

Em resumo, o problema de corte bidimensional não-guilhotinado multiperíodo pode ser modelado minimizando a função objetivo (2.21) nas variáveis $u_{s j} \in\{0,1\}\left(s=p, \ldots, P, j=1, \ldots, \bar{m}_{s}\right), v_{s i j} \in$ $\{0,1\}\left(s=p, \ldots, P, i=1, \ldots, n_{s}, j=1, \ldots, \bar{m}_{s}\right),\left(x_{s i}, y_{s i}\right) \in \mathbb{R}^{2}\left(s=p, \ldots, P, i=1, \ldots, n_{s}\right)$, $\pi_{s i i^{\prime}} \in\{0,1\}\left(s=p, \ldots, P, i=1, \ldots, n_{s}, i^{\prime}=i+1, \ldots, n_{s}\right), \tau_{s i i^{\prime}} \in\{0,1\}\left(s=p, \ldots, P, q_{s}=\right.$ $\left.1, \ldots, \tilde{n}_{s}, i=o_{s q}+1, \ldots, o_{s q}+z_{s q}, i^{\prime}=i+1, \ldots, n_{s}\right), \eta_{s j} \in\{0,1\}\left(s=p, \ldots, P, j=1, \ldots, \bar{m}_{s}\right)$, $r_{s j}, t_{s j}, \bar{W}_{s j}, \bar{H}_{s j} \in \mathbb{R}\left(s=p, \ldots, P, j=1, \ldots, \bar{m}_{s}\right)$ sujeita à $(2.19,2.20,2.22,2.25,2.27,2.28,2.29$, $2.30,2.31,2.32,2.33,2.34)$. Entretanto, como mostrado na seção anterior, podemos ter soluções com o mesmo custo, mas com diferentes sobras remanescentes no último período, que poderiam ser utilizadas futuramente. Dessa forma, estamos interessados em soluções que minimizam o custo dos objetos usados para cortar todos os itens da ordem de trabalho de todos os períodos do horizonte de planejamento, e, dentre todas as soluções de custo mínimo, aquelas que maximizam o valor das sobras aproveitáveis remanescentes no último período, que podem ser sobras dos objetos usados no último período ou sobras originadas de objetos de períodos anteriores.

Novamente, supomos que dada uma lista contendo $d \geq 1$ de itens (itens do catálogo), com larguras $\bar{w}_{1}, \ldots, \bar{w}_{d}$ e alturas $\bar{h}_{1}, \ldots, \bar{h}_{d}$, uma sobra é considerada aproveitável se suas dimensões permitem o corte de ao menos um item do catálogo. Sejam $\alpha_{P j}^{\mathrm{t}}$ e $\alpha_{P j}^{\mathrm{r}}$ respectivamente o valor da área da sobra superior e da sobra do lado direito do objeto $j$ do último período para o Caso A, e sejam $\beta_{P j}^{\mathrm{t}}$ e $\beta_{P j}^{\mathrm{r}}$ o valor da área da sobra superior e da sobra do lado direito, respectivamente, do objeto $j$ do último período para o Caso B, com $j=1, \ldots, \bar{m}_{P}$. Como mostrado pela Figura $2.7, \alpha_{P j}^{\mathrm{t}}, \alpha_{P j}^{\mathrm{r}}$, $\beta_{P j}^{\mathrm{t}}$ e $\beta_{P j}^{\mathrm{r}}$ são dados por 


$$
\begin{aligned}
& \alpha_{P j}^{\mathrm{t}}=\left\{\begin{aligned}
\left(\bar{W}_{P j}-r_{P j}\right) t_{P j}, & \text { se existe } 1 \leq i \leq d \text { tal que } \bar{W}_{P j}-r_{P j} \geq \bar{w}_{i} \text { e } t_{P j} \geq \bar{h}_{i}, \\
0, & \text { caso contrário, }
\end{aligned}\right. \\
& \alpha_{P j}^{\mathrm{r}}=\left\{\begin{array}{rl}
r_{P j} \bar{H}_{P j}, & \text { se existe } 1 \leq i \leq d \text { tal que } r_{P j} \geq \bar{w}_{i} \text { e } \bar{H}_{P j} \geq \bar{h}_{i}, \\
0, & \text { caso contrário, }
\end{array} \quad j=1, \ldots, \bar{m}_{P} .\right. \\
& \beta_{P j}^{\mathrm{t}}=\left\{\begin{aligned}
\bar{W}_{P j} t_{P j}, & \text { se existe } 1 \leq i \leq d \text { tal que } \bar{W}_{P j} \geq \bar{w}_{i} \text { e } t_{P j} \geq \bar{h}_{i}, \\
0, & \text { caso contrário, }
\end{aligned}\right. \\
& \beta_{P j}^{\mathrm{r}}=\left\{\begin{aligned}
r_{P j}\left(\bar{H}_{P j}-t_{P j}\right), & \text { se existe } 1 \leq i \leq d \text { tal que } r_{P j} \geq \bar{w}_{i} \text { e } \bar{H}_{P j}-t_{P j} \geq \bar{h}_{i}, \\
0, & \text { caso contrário. }
\end{aligned}\right.
\end{aligned}
$$

No último período, devemos saber a qual objeto de entrada cada sobra pertence, para que cada uma tenha seu custo corretamente estabelecido. Seja $\bar{c}_{j}$ o custo do objeto $j$, onde os primeiros $m_{P}$ valores são os custos dos objetos do último período e os $\bar{m}_{P}-m_{P}$ restantes (objetos originados de sobras) estão associados aos custos dos objetos dos quais estes objetos foram originados. Dessa forma, a soma dos valores das sobras aproveitáveis é dada por

$$
\sum_{j=1}^{\bar{m}_{P}} \bar{c}_{j} \max \left\{\alpha_{P j}^{\mathrm{t}}+\alpha_{P j}^{\mathrm{r}}, \beta_{P j}^{\mathrm{t}}+\beta_{P j}^{\mathrm{r}}\right\} .
$$

Definindo as variáveis contínuas $\gamma_{P j}^{\mathrm{r}}$ e $\gamma_{P j}^{\mathrm{t}}$ para representarem, respectivamente, a área da sobra da direita e a área da sobra superior do período $P$, para $j=1, \ldots, \bar{m}_{P}$, e adicionando as restrições

$$
\begin{aligned}
\gamma_{P j}^{\mathrm{r}} & \leq \alpha_{P j}^{\mathrm{r}}+\hat{W} \eta_{P j}, \\
\gamma_{P j}^{\mathrm{t}} & \leq \alpha_{P j}^{\mathrm{t}}+\hat{H} \eta_{P j}, \\
\gamma_{P j}^{\mathrm{r}} & \leq \beta_{P j}^{\mathrm{r}}+\hat{W}\left(1-\eta_{P j}\right), \\
\gamma_{P j}^{\mathrm{t}} & \leq \beta_{P j}^{\mathrm{t}}+\hat{H}\left(1-\eta_{P j}\right),
\end{aligned}
$$

a função objetivo (2.36) pode ser reescrita como uma função linear dada por

$$
\sum_{j=1}^{\bar{m}_{P}} \bar{c}_{j}\left(\gamma_{P j}^{\mathrm{r}}+\gamma_{P j}^{\mathrm{t}}\right) .
$$

Para modelar os produtos $r_{P j} t_{P j}, r_{P j} \bar{H}_{P j}$ e $\bar{W}_{P j} t_{P j}$, presentes nas definições de $\alpha_{P j}^{\mathrm{t}}, \alpha_{P j}^{\mathrm{r}}, \beta_{P j}^{\mathrm{t}}$ e $\beta_{P j}^{\mathrm{r}}$ em (2.35), usando restrições MIP, podemos substituir as variáveis $r_{P j}$ e $t_{P j}$ por suas representações binárias reduzindo cada um dos produtos em uma soma de produtos de variáveis inteiras e variáveis binárias, exceto em $r_{P j} t_{P j}$, que é reduzido a uma soma de produtos de variáveis binárias. Sejam $\theta_{P j \ell}^{\mathrm{r}}\left(j=1, \ldots, \bar{m}_{P}, \ell=1, \ldots,\left\lfloor\log _{2}(\hat{W})\right\rfloor+1\right)$ e $\theta_{P j \ell}^{\mathrm{t}}\left(j=1, \ldots, \bar{m}_{P}, \ell=1, \ldots,\left\lfloor\log _{2}(\hat{H})\right\rfloor+1\right)$ variáveis binárias usadas para a representação binária de $r_{P j}$ e $t_{P j}$, respectivamente. Perceba que $\hat{W}$ e $\hat{H}$ são limitantes superiores de $r_{P j}$ e $t_{P j}$, respectivamente, e que $\left\lfloor\log _{2}(\hat{W})\right\rfloor+1$ e $\left\lfloor\log _{2}(\hat{H})\right\rfloor+1$ 
são respectivamente limitantes superiores para o número de bits necessários para a representação binária de $r_{P j}$ e $t_{P j}$, com $j=1, \ldots, \bar{m}_{P}$. Dessa forma, $r_{P j}$ e $t_{P j}$ podem ser expressadas como:

$$
\begin{aligned}
r_{P j} & =\sum_{\ell=1}^{\left\lfloor\log _{2}(\hat{W})\right\rfloor+1} 2^{\ell-1} \theta_{P j \ell}^{\mathrm{r}}, \quad j=1, \ldots, \bar{m}_{P}, \\
t_{P j} & =\sum_{\ell=1}^{\left\lfloor\log _{2}(\hat{H})\right\rfloor+1} 2^{\ell-1} \theta_{P j \ell}^{\mathrm{t}}, \quad j=1, \ldots, \bar{m}_{P},
\end{aligned}
$$

e, assim, cada produto $r_{P j} t_{P j}, r_{P j} \bar{H}_{P j}$ e $\bar{W}_{P j} t_{P j}$ pode ser expressado como

$$
\begin{aligned}
r_{P j} t_{P j} & =\sum_{\ell=1}^{\left\lfloor\log _{2}(\hat{H})\right\rfloor+1} 2^{\ell-1} r_{P j} \theta_{P j \ell}^{\mathrm{t}}, \\
r_{P j} \bar{H}_{P j} & =\sum_{\ell=1}^{\left\lfloor\log _{2}(\hat{W})\right\rfloor+1} 2^{\ell-1} \bar{H}_{P j} \theta_{P j \ell}^{\mathrm{r}}, \quad j=1, \ldots, \bar{m}_{P} . \\
\bar{W}_{P j} t_{P j} & =\sum_{\ell=1}^{\left\lfloor\log _{2}(\hat{H})\right\rfloor+1} 2^{\ell-1} \bar{W}_{P j} \theta_{P j \ell}^{\mathrm{t}},
\end{aligned}
$$

Dessa forma, o produto de duas variáveis inteiras é reduzido à soma dos produtos de uma variável inteira e uma variável binária. O valor de cada um desses produtos é igual a zero quando a variável binária é igual a zero e coincide com o valor da variável inteira quando a variável binária é igual a um. Considerando as variáveis contínuas $\omega_{P j \ell}^{\mathrm{rt}}, \omega_{P j \ell}^{\mathrm{Wt}}, j=1, \ldots, \bar{m}_{P}, \ell=1, \ldots,\left\lfloor\log _{2}(\hat{H})\right\rfloor+1$ e $\omega_{P j \ell}^{\mathrm{rH}}$, $j=1, \ldots, \bar{m}_{P}, \ell=1, \ldots,\left\lfloor\log _{2}(\hat{W})\right\rfloor+1$, essa situação pode ser modelada usando restrições que garantem que:

$$
\begin{aligned}
\omega_{P j \ell}^{\mathrm{rt}} & =\left\{\begin{aligned}
r_{P j}, & \text { sempre que } \theta_{P j \ell}^{\mathrm{t}}=1, \\
0, & \text { caso contrário, }
\end{aligned}\right. \\
\omega_{P j \ell}^{\mathrm{Wt}} & =\left\{\begin{aligned}
\bar{W}_{P j}, & \text { sempre que } \theta_{P j \ell}^{\mathrm{t}}=1, \\
0, & \text { caso contrário, }
\end{aligned}\right. \\
\omega_{P j \ell}^{\mathrm{rH}} & =\left\{\begin{aligned}
\bar{H}_{P j}, & \text { sempre que } \theta_{P j \ell}^{\mathrm{r}}=1, \\
0, & \text { caso contrário. }
\end{aligned}\right.
\end{aligned}
$$

Restrições MIP que modelam as condições acima são dadas por: 


$$
\begin{aligned}
0 & \leq \omega_{P j \ell}^{\mathrm{rt}} \leq r_{P j}, \quad j=1, \ldots, \bar{m}_{P}, \ell=1, \ldots,\left\lfloor\log _{2}(\hat{H})\right\rfloor+1, \\
r_{P j}-\left(1-\theta_{P j \ell}^{\mathrm{t}}\right) \hat{W} & \leq \omega_{P j \ell}^{\mathrm{rt}} \leq \theta_{P j \ell}^{\mathrm{t}} \hat{W}, \quad j=1, \ldots, \bar{m}_{P}, \ell=1, \ldots,\left\lfloor\log _{2}(\hat{H})\right\rfloor+1, \\
0 & \leq \omega_{P j \ell}^{\mathrm{Wt}} \leq \bar{W}_{P j}, \quad j=1, \ldots, \bar{m}_{P}, \ell=1, \ldots,\left\lfloor\log _{2}(\hat{H})\right\rfloor+1, \\
\bar{W}_{P j}-\left(1-\theta_{P j \ell}^{\mathrm{t}}\right) \hat{W} & \leq \omega_{P j \ell}^{\mathrm{Wt}} \leq \theta_{P j \ell}^{\mathrm{t}} \hat{W}, \quad j=1, \ldots, \bar{m}_{P}, \ell=1, \ldots,\left\lfloor\log _{2}(\hat{H})\right\rfloor+1, \\
0 & \leq \omega_{P j \ell}^{\mathrm{rH}} \leq \bar{H}_{P j}, j=1, \ldots, \bar{m}_{P}, \ell=1, \ldots,\left\lfloor\log _{2}(\hat{W})\right\rfloor+1, \\
\bar{H}_{P j}-\left(1-\theta_{P j \ell}^{\mathrm{r}}\right) \hat{H} & \leq \omega_{P j \ell}^{\mathrm{rH}} \leq \theta_{P j \ell}^{\mathrm{r}} \hat{H}, \quad j=1, \ldots, \bar{m}_{P}, \ell=1, \ldots,\left\lfloor\log _{2}(\hat{W})\right\rfloor+1 .
\end{aligned}
$$

Assim, temos que todas as variáveis $r_{P j}$ e $t_{P j}$ podem ser substituídas por suas representações binárias (2.39) e (2.40), respectivamente, e todos os produtos $r_{P j} t_{P j}, \bar{W}_{P j} t_{P j}$ e $r_{P j} \bar{H}_{P j}$ (nas definições de $\alpha_{P j}^{\mathrm{t}}, \alpha_{P j}^{\mathrm{r}}, \beta_{P j}^{\mathrm{t}}$ e $\beta_{P j}^{\mathrm{r}}$ em (2.35)) podem ser substituídos por suas reformulações MIP dadas, respectivamente, por:

$$
\begin{aligned}
r_{P j} t_{P j} & =\sum_{\ell=1}^{\left\lfloor\log _{2}(\hat{H})\right\rfloor+1} 2^{\ell-1} \omega_{P j \ell}^{\mathrm{rt}}, \quad j=1, \ldots, \bar{m}_{P}, \\
\bar{W}_{P j} t_{P j} & =\sum_{\ell=1}^{\left\lfloor\log _{2}(\hat{H})\right\rfloor+1} 2^{\ell-1} \omega_{P j \ell}^{\mathrm{Wt}}, \quad j=1, \ldots, \bar{m}_{P}, \\
r_{P j} \bar{H}_{P j} & =\sum_{\ell=1}^{\left\lfloor\log _{2}(\hat{W})\right\rfloor+1} 2^{\ell-1} \omega_{P j \ell}^{\mathrm{rH}}, \quad j=1, \ldots, \bar{m}_{P} .
\end{aligned}
$$

De forma similar ao apresentado na seção anterior, para modelar o conjunto de restrições (2.35) como restrições MIP, sejam $\bar{\alpha}_{P i j}^{\mathrm{t}}, \bar{\alpha}_{P i j}^{\mathrm{r}}, \bar{\beta}_{P i j}^{\mathrm{t}}, \bar{\beta}_{P i j}^{\mathrm{r}} \in\{0,1\}, i=1, \ldots, d, j=1, \ldots, \bar{m}_{P}$, e considere as restrições:

$$
\begin{array}{rlrl}
\bar{w}_{i} \leq \bar{W}_{P j}-r_{P j}+\hat{W}\left(1-\bar{\alpha}_{P i j}^{\mathrm{t}}\right) & \text { e } & \bar{h}_{i} \leq t_{P j}+\hat{H}\left(1-\bar{\alpha}_{P i j}^{\mathrm{t}}\right), \\
\bar{w}_{i} \leq r_{P j}+\hat{W}\left(1-\bar{\alpha}_{P i j}^{\mathrm{r}}\right) & \text { e } & \bar{h}_{i} \leq \bar{H}_{P j}+\hat{H}\left(1-\bar{\alpha}_{P i j}^{\mathrm{r}}\right), \\
\bar{w}_{i} \leq \bar{W}_{P j}+\hat{W}\left(1-\bar{\beta}_{P i j}^{\mathrm{t}}\right) & \mathrm{e} & \bar{h}_{i} \leq t_{P j}+\hat{H}\left(1-\bar{\beta}_{P i j}^{\mathrm{t}}\right), \\
\bar{w}_{i} \leq r_{P j}+\hat{W}\left(1-\bar{\beta}_{P i j}^{\mathrm{r}}\right) & \mathrm{e} & \bar{h}_{i} \leq & \bar{H}_{P j}-t_{P j}+\hat{H}\left(1-\bar{\beta}_{P i j}^{\mathrm{r}}\right), \\
& & & i=1, \ldots, d, j=1, \ldots, \bar{m}_{P},
\end{array}
$$

$\mathrm{e}$

$$
\begin{array}{rlll}
0 & \leq \alpha_{P j}^{\mathrm{t}} \leq \bar{W}_{P j} t_{P j}-r_{P j} t_{P j} & \text { e } & \alpha_{P j}^{\mathrm{t}} \leq \hat{W} \hat{H} \sum_{i=1}^{d} \bar{\alpha}_{P i j}^{T m}, \\
0 & \leq \alpha_{P j}^{\mathrm{r}} \leq r_{P j} \bar{H}_{P j} & \text { e } & \alpha_{P j}^{\mathrm{r}} \leq \hat{W} \hat{H} \sum_{i=1}^{d} \bar{\alpha}_{P i j}^{\mathrm{r}}, \\
0 & \leq \beta_{P j}^{\mathrm{t}} \leq \bar{W}_{P j} t_{P j} & \text { e } & \beta_{P j}^{\mathrm{t}} \leq \hat{W} \hat{H} \sum_{i=1}^{d} \bar{\beta}_{P i j}^{\mathrm{t}}, \\
0 & \leq \beta_{P j}^{\mathrm{r}} \leq r_{P j} \bar{H}_{P j}-r_{P j} t_{P j} \quad \text { e } & \beta_{P j}^{\mathrm{r}} \leq \hat{W} \hat{H} \sum_{i=1}^{d} \bar{\beta}_{P i j}^{\mathrm{r}},
\end{array}
$$


Por questão de simplicidade, as variáveis $r_{P j}$ e $t_{P j}$ não foram substituídas por suas respectivas representações binárias, (2.39) e (2.40), no texto. O mesmo acontece com os produtos $r_{P j} t_{P j}, \bar{W}_{P j} t_{P j}$ e $r_{P j} \bar{H}_{P j}$, que devem ser substituídos por suas respectivas representações MIP, dadas por (2.42), (2.43) e (2.44). Quando o $i$-ésimo item do catálogo não couber dentro da sobra superior do Caso A, na sobra do lado direito do Caso A, na sobra superior do Caso B e nem na sobra do lado direito do Caso $\mathrm{B}$, respectivamente, as variáveis $\bar{\alpha}_{P i j}^{\mathrm{t}}, \bar{\alpha}_{P i j}^{\mathrm{r}}, \bar{\beta}_{P i j}^{\mathrm{t}}$ e $\bar{\beta}_{P i j}^{\mathrm{r}}$ devem ser nulas. Isso é garantido pelo conjunto de restrições (2.45). Caso contrário, as variáveis ficam livres para assumirem zero ou um. Dessa forma, o conjunto de restrições (2.46) garante que as variáveis $\alpha_{P j}^{\mathrm{t}}, \alpha_{P j}^{\mathrm{r}}$, $\beta_{P j}^{\mathrm{t}}$ e $\beta_{P j}^{\mathrm{r}}$ serão nulas quando na sobra correspondente não couber nenhum item do catálogo. Caso contrário, elas ficam livres e podem assumir qualquer valor entre zero e a área da sobra correspondente. Uma vez que essas variáveis limitam o crescimento de $\gamma_{P j}^{\mathrm{r}}$ e $\gamma_{P j}^{\mathrm{t}}$ em (2.37) e a função objetivo (2.38) maximiza a combinação linear não-negativa de $\gamma_{P j}^{\mathrm{r}}$ e $\gamma_{P j}^{\mathrm{t}}$, então, em uma solução, as variáveis $\alpha_{P j}^{\mathrm{t}}, \alpha_{P j}^{\mathrm{r}}$, $\beta_{P j}^{\mathrm{t}}$ e $\beta_{P j}^{\mathrm{r}}$ atingirão seus valores máximos possíveis, que irão coincidir com a área das sobras aproveitáveis remanescentes do último período.

Finalmente estamos prontos para formular um modelo para o problema de corte bidimensional multiperíodo com sobras aproveitáveis (PCBMPSA), que consiste em cortar os itens das ordens de trabalho de todo o horizonte considerado usando, em cada período, objetos de entrada disponíveis no período ou sobras de períodos anteriores. O custo dos objetos utilizados é minimizado e, dentre todas as possíveis soluções de custo mínimo, escolhe-se aquela que, no fim do horizonte de tempo considerado, maximiza o valor das sobras aproveitáveis remanescentes. Novamente, temos um Problema de Programação Dois Níveis, o qual pode ser reformulado como um modelo de Programação Linear Inteira Mista uma vez que as funções objetivos (2.21) e (2.38) assumem apenas valores inteiros e possuem limitantes superiores e inferiores triviais. Portanto, a função objetivo do problema de nível superior (2.38) pode ser usada como desempate entre todas as possíveis soluções ótimas do problema de nível inferior (2.21). É fácil perceber que a função objetivo (2.38) assume valores inteiros, que são maiores ou iguais a zero e estritamente menores que $\sum_{s=p}^{P} \sum_{j=1}^{m_{s}} c_{s j} W_{s j} H_{s j}$. Assim, a combinação adequada das funções objetivos (2.38) e (2.21) é dada por

$$
\left(\sum_{s=p}^{P} \sum_{j=1}^{m_{s}} c_{s j} W_{s j} H_{s j}\right)\left(\sum_{s=p}^{P} \sum_{j=1}^{m_{s}} c_{s j} W_{s j} H_{s j} u_{s j}\right)-\sum_{j=1}^{\bar{m}^{P}} \bar{c}_{j}\left(\gamma_{P j}^{\mathrm{r}}+\gamma_{P j}^{\mathrm{t}}\right)
$$

Como o termo relacionado à função objetivo (2.21) é multiplicado por um limitante superior estrito da função objetivo (2.38), temos que cada unidade do termo relacionado à função objetivo (2.21) é mais importante que todo o valor do termo relacionado com a função objetivo (2.38). Assim, ao minimizar (2.47), o termo relacionado à função objetivo (2.38) participa no desempate das soluções que minimizam (2.21), escolhendo aquela com máximo valor de sobras aproveitáveis remanescentes.

Agora podemos apresentar o modelo do problema de corte bidimensional multiperíodo com sobras aproveitáveis (PCBMPSA). As variáveis do problema são: $u_{s j} \in\{0,1\}(s=p, \ldots, P, j=$ $\left.1, \ldots, \bar{m}_{s}\right)$ para distinguir objetos usados e não usados; $v_{s i j} \in\{0,1\}(s=p, \ldots, P, i=1, \ldots, n, j=$ $\left.1, \ldots, \bar{m}_{s}\right)$ para definir em qual objeto cada item deve estar; $\left(x_{s i}, y_{s i}\right) \in \mathbb{R}^{2}(s=p, \ldots, P, i=$ 
$\left.1, \ldots, n_{s}\right)$ para definir a posição dos itens dentro dos objetos; $\pi_{s i i^{\prime}} \in\{0,1\}(s=p, \ldots, P, i=$ $\left.1, \ldots, n_{s}, i^{\prime}=i+1, \ldots, n_{s}\right)$ e $\tau_{s i i^{\prime}} \in\{0,1\}\left(s=p, \ldots, P, q_{s}=1, \ldots, \tilde{n}_{s}, i=o_{s q}+1, \ldots, o_{s q}+\right.$ $\left.z_{s q}, i^{\prime}=i+1, \ldots, n_{s}\right)$ para modelar e impedir a sobreposição dos itens; $\eta_{s j} \in\{0,1\}(s=p, \ldots, P, j=$ $1, \ldots, \bar{m}_{s}$ ) para selecionar o tipo de corte guilhotinado (Caso A ou Caso B) para gerar as sobras de cada objeto; $\gamma_{P j}^{\mathrm{r}}$ e $\gamma_{P j}^{\mathrm{t}} \in \mathbb{R}\left(j=1, \ldots, \bar{m}_{P}\right)$ para representar a área da sobra da direita e da sobra superior, respectivamente, do tipo de corte com melhor sobra aproveitável dos objetos do último período; $r_{s j} \mathrm{e}$ $t_{s j}$ para descrever, respectivamente, a altura da sobra superior e a largura do lado direito do objeto $j$ no período $s$, para $s=1, \ldots, P-1, j=1, \ldots, \bar{m}_{s} ; \bar{W}_{s j}$ e $\bar{H}_{s j} \in \mathbb{R}\left(s=p, \ldots, P, j=1, \ldots, \bar{m}_{s}\right)$ para representar as dimensões dos objetos, sendo que, em cada período, $\bar{W}_{s j}$ e $\bar{H}_{s j}$ coincidem com $W_{s j}$ e $H_{s j}$, respectivamente, para $s=p, \ldots, P, j=1, \ldots, m_{s}$ e para $s=p+1, \ldots, P, j=m_{s}+1, \ldots, \bar{m}_{s}, \bar{W}_{s j}$ e $\bar{H}_{s j}$ serão exatamente as dimensões das sobras dos objetos do período anterior; $\theta_{P j \ell}^{\mathrm{r}} \in\{0,1\}(j=$ $\left.1, \ldots, \bar{m}_{P}, \ell=1, \ldots,\left\lfloor\log _{2}(\hat{W})\right\rfloor+1\right)$ e $\theta_{P j \ell}^{\mathrm{t}} \in\{0,1\}\left(j=1, \ldots, \bar{m}_{P}, \ell=1, \ldots,\left\lfloor\log _{2}(\hat{H})\right\rfloor+1\right)$ para modelar a representação binária respectivamente da largura máxima da sobra do lado direito e a altura máxima da sobra superior dos objetos; $\omega_{P j \ell}^{\mathrm{rt}} \in \mathbb{R}\left(j=1, \ldots, \bar{m}_{P}, \ell=1, \ldots,\left\lfloor\log _{2}(\hat{H})\right\rfloor+1\right), \omega_{P j \ell}^{\mathrm{Wt}} \in$ $\mathbb{R}\left(j=1, \ldots, \bar{m}_{P}, \ell=1, \ldots,\left\lfloor\log _{2}(\hat{H})\right\rfloor+1\right)$ e $\omega_{P j \ell}^{\mathrm{rH}} \in \mathbb{R}\left(j=1, \ldots, \bar{m}_{P}, \ell=1, \ldots,\left\lfloor\log _{2}(\hat{W})\right\rfloor+1\right)$ para modelar os produtos que aparecem quando é calculada a área das sobras de cada objeto do último período; $\alpha_{P j}^{\mathrm{t}}, \alpha_{P j}^{\mathrm{r}}, \beta_{P j}^{\mathrm{t}} \mathrm{e} \beta_{P j}^{\mathrm{r}} \in \mathbb{R},\left(j=1, \ldots, \bar{m}_{P}\right)$ para representar a área das sobras aproveitáveis dos objetos no último período; e $\bar{\alpha}_{P i j}^{\mathrm{t}}, \bar{\alpha}_{P i j}^{\mathrm{r}}, \bar{\beta}_{P i j}^{\mathrm{t}}$ e $\bar{\beta}_{P i j}^{\mathrm{r}} \in\{0,1\},\left(i=1, \ldots, d, j=1, \ldots, \bar{m}_{P}\right)$ para indicar se a sobra, do último período, comporta algum item do catálogo.

A formulação do problema consiste em minimizar (2.47) nas variáveis $u_{s j}, v_{s i j}, x_{s i}, y_{s i}, \pi_{s i i^{\prime}}, \tau_{s i i^{\prime}}$, $\eta_{s j}, \gamma_{P j}^{\mathrm{r}}, \gamma_{P j}^{\mathrm{t}}, \bar{W}_{j}^{s}, \bar{H}_{j}^{s}, \theta_{P j \ell}^{r}, \theta_{P j \ell}^{\mathrm{t}}, \omega_{P j \ell}^{\mathrm{rt}}, \omega_{P j \ell}^{\mathrm{Wt}}, \omega_{P j \ell}^{\mathrm{rH}}, \alpha_{P j}^{\mathrm{t}}, \alpha_{P j}^{\mathrm{r}}, \beta_{P j}^{\mathrm{t}}, \beta_{P j}^{\mathrm{r}}, \bar{\alpha}_{P i j}^{\mathrm{t}}, \bar{\alpha}_{P i j}^{\mathrm{r}}, \bar{\beta}_{P i j}^{\mathrm{t}} \mathrm{e} \bar{\beta}_{P i j}^{\mathrm{r}}$ sujeita à $(2.19$, 2.20, 2.22, 2.25, 2.27, 2.28, 2.29, 2.30, 2.31, 2.32, 2.33, 2.34, 2.37, 2.41, 2.45, 2.46). Perceba que as variáveis $\gamma_{P j}^{\mathrm{r}}, \gamma_{P j}^{\mathrm{t}}, \theta_{P j \ell}^{\mathrm{r}}, \theta_{P j \ell}^{\mathrm{t}}, \omega_{P j \ell}^{\mathrm{rt}}, \omega_{P j \ell}^{\mathrm{Wt}}, \omega_{P j \ell}^{\mathrm{rH}}, \alpha_{P j}^{\mathrm{t}}, \alpha_{P j}^{\mathrm{r}}, \beta_{P j}^{\mathrm{t}}, \beta_{P j}^{\mathrm{r}}, \bar{\alpha}_{P i j}^{\mathrm{t}}, \bar{\alpha}_{P i j}^{\mathrm{r}}, \bar{\beta}_{P i j}^{\mathrm{t}}$ e $\bar{\beta}_{P i j}^{\mathrm{r}}$ são definidas apenas para o último período $P$. Note também que toda aparição de $r_{P j}$ e $t_{P j}$ na formulação deve ser substituída por suas representações binárias, definidas respectivamente em (2.39) e (2.40) e os produtos $r_{P j} t_{P j}$, $\bar{W}_{P j} t_{P j}$ e $r_{P j} \bar{H}_{P j}$ devem ser substituídos por suas respectivas representações MIP, dadas por (2.42), (2.43) e (2.44). Chamamos esse modelo de $\mathcal{M}_{p, P}^{1}$. Perceba que o problema de corte bidimensional multiperíodo com sobras aproveitáveis PCBMPSA é uma generalização do PCSA, apresentado na seção anterior, ou seja, se $p=P$ então o PCBMPSA se reduz ao PCSA.

Como exemplo ilustrativo, considere uma instância com $p=1$ e $P=2 ; m_{1}=2$ e $m_{2}=1$, com $W_{11}=10, H_{11}=7, W_{12}=12, H_{12}=6, W_{21}=12, H_{21}=8$ e $c_{11}=c_{12}=c_{21}=1$; e $n_{1}=n_{2}=2$, $\operatorname{com} w_{11}=w_{12}=5, h_{11}=h_{12}=7, w_{21}=7, h_{21}=6, w_{22}=5$ e $h_{22}=3$. A Figura 2.11 mostra os objetos e itens de cada período. Além disso, considere que o catálogo contenha apenas o item $5 \times 3$, ou seja, qualquer sobra em que caiba um item de $5 \times 3$ será considerada uma sobra aproveitável. A Figura 2.12 apresenta uma solução ótima encontrada pelo CPLEX considerando o modelo $\mathcal{M}_{p, P}^{1}$. Observe que o objeto $O_{12}$ foi comprado (no período 1), mas nenhum item da ordem de trabalho do período 1 foi atribuído a ele. Além disso, note que o modelo permitiu que houvesse um corte nesse objeto, produzindo duas sobras aproveitáveis. Analisando essa solução, observamos que três opções diferentes poderiam ter sido consideradas na modelagem do problema proposto: $(i)$ permitir a compra e o eventual corte de objetos de entrada em um determinado período, mesmo que nenhum 
item no período seja atribuído ao objeto, o que é feito no modelo $\mathcal{M}_{p, P}^{1}$; (ii) permitir a compra de objetos de entrada que não contenham itens em um período, mas sem permitir que tais objetos sejam cortados, ou seja, ou a área da sobra aproveitável da direita ou a área da sobra aproveitável superior deve coincidir com a área do objeto; e (iii) impedir que objetos aos quais não serão atribuídos itens sejam comprados.

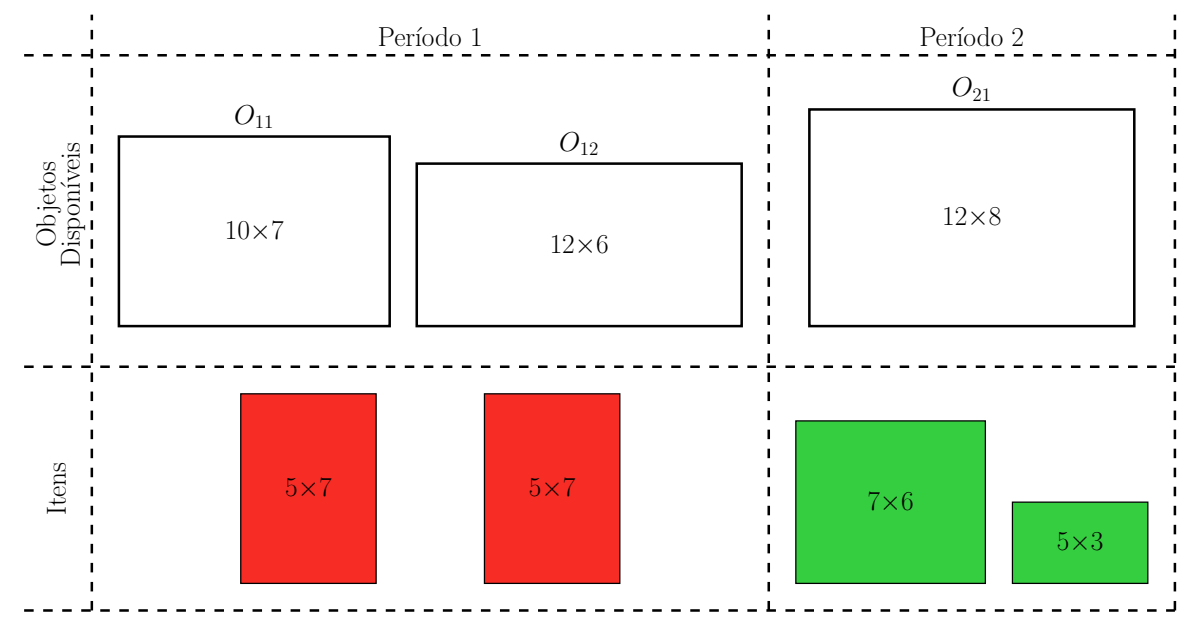

Figura 2.11: Instância para exemplificar três estratégias para lidar com objetos selecionados sem itens atribuídos.

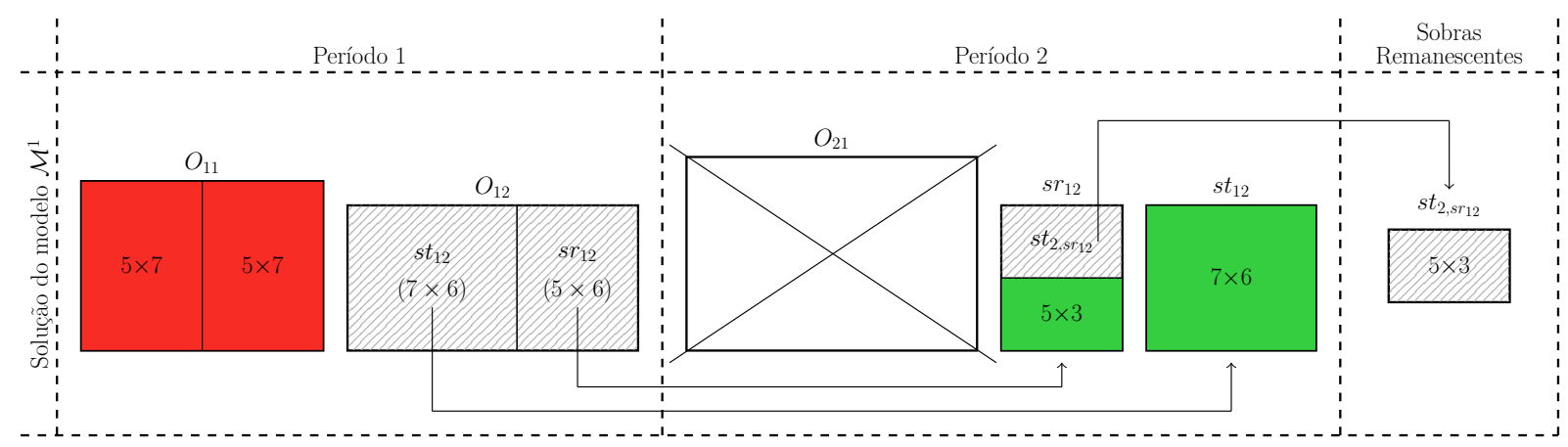

Figura 2.12: Solução da instância da Figura 2.11 usando o modelo $\mathcal{M}_{p, P}^{1}$. Observe que o objeto $O_{12}$ não possui itens no primeiro período e, além disso, foi dividido em duas sobras de forma que seja possível gerar uma sobra aproveitável final. A solução possui custo dos objetos utilizados igual a 142 e gerou uma sobra aproveitável final de valor 15.

Para impedir cortes em um objeto (comprado) utilizado, mas ao qual não sejam atribuídos itens, opção (ii), devemos garantir que a área da sobra, da direita ou superior, coincida com a área do objeto. Por escolha, vamos considerar que, neste caso, a área da sobra da direita deverá ser igual a área do objeto, isto é, $r_{s j}=W_{s j}$ e $t_{s j}=0$ sempre que $u_{s j}=1$ e $\sum_{i=1}^{n_{s}} v_{s i j}=0$, para $s=p, \ldots, P$, $j=1, \ldots, m_{s}$. Para garantir isso, devemos ter: 


$$
\begin{array}{ll}
r_{s j} \geq\left(u_{s j}-\sum_{i=1}^{n_{s}} v_{s i j}\right) W_{s j}, & s=p, \ldots, P, j=1, \ldots, m_{s}, \\
t_{s j} \leq H_{s j} \sum_{i=1}^{n_{s}} v_{s i j}, & s=p, \ldots, P, j=1, \ldots, m_{s} .
\end{array}
$$

As restrições (2.48), em conjunto com as restrições (2.25), garantem que $r_{s j}$ será igual a $W_{s j}$ sempre que $u_{s j}=1$ e $\sum_{i=1}^{n_{s}} v_{s i j}=0$. Se $\sum_{i=1}^{n_{s}} v_{s i j} \geq 1$, então $u_{s j}=1$, devido as restrições (2.19), e assim teremos, do lado direito, um valor menor ou igual a zero, mas as restrições (2.25) garantem que $0 \leq r_{s j} \leq W_{s j}$. Já o conjunto de restrições (2.48) força $t_{s j}=0$ sempre que não houver nenhum item atribuído ao objeto $j$ no período $s$. Caso o objeto tenha algum item atribuído $\left(\sum_{i=1}^{n_{s}} v_{s i j} \geq 1\right), t_{s j}$ fica livre para ser qualquer valor entre zero e $H_{s j}$ (altura do objeto $j$ do período $s$ ), o que é garantido pelas restrições (2.25). Combinando as restrições do modelo $\mathcal{M}_{p, P}^{1}$ com as restrições (2.48) e (2.49), definimos o modelo $\mathcal{M}_{p, P}^{2}$. A Figura 2.13 ilustra a solução da instância da Figura 2.11 considerando o modelo $\mathcal{M}_{p, P}^{2}$. Como o objeto $O_{12}$ não pode ser cortado e seguindo o padrão adotado para sobra, no segundo período não é possível gerar uma sobra aproveitável remanescente, como aconteceu na solução do modelo $\mathcal{M}_{p, P}^{1}$. Note que, caso tenhamos um terceiro período com um único item de tamanho $5 \times 3$, o modelo $\mathcal{M}_{p, P}^{2}$ precisaria utilizar um novo objeto para cortá-lo, enquanto o modelo $\mathcal{M}_{p, P}^{1}$ usaria a sobra aproveitável, gerada no segundo período, e não precisaria comprar um novo objeto.

$\mathrm{Na}$ alternativa (iii) impedimos que, em um determinado período, sejam comprados objetos que não serão utilizados no período. Isso pode ser modelado da seguinte forma,

$$
u_{s j} \leq \sum_{i=1}^{n_{s}} v_{s i j}, \quad s=p, \ldots, P, j=1, \ldots, m_{s} .
$$

Este conjunto de restrições garante que um objeto só será selecionado caso haja a atribuição de algum item a ele. Adicionando a restrição (2.50) ao modelo $\mathcal{M}_{p, P}^{1}$, compomos o modelo $\mathcal{M}_{p, P}^{3}$. A Figura 2.14 ilustra a solução do exemplo anterior considerando o modelo $\mathcal{M}_{p, P}^{3}$. No primeiro período, apenas o objeto $O_{11}$ é utilizado. No segundo período, como não existe objeto originado de sobra, os itens devem ser cortados no único objeto disponível do período, que é mais caro que o objeto $O_{12}$ do primeiro período e, consequentemente, aumenta o custo da solução.

Por acharmos mais natural, o modelo $\mathcal{M}_{p, P}^{2}$ será o modelo adotado nos capítulos seguintes e, quando não dito de forma diferente, consideraremos $\xi=P-p$, de forma que uma sobra pode estar disponível, para ser utilizada, em qualquer período posterior ao qual foi gerada. 


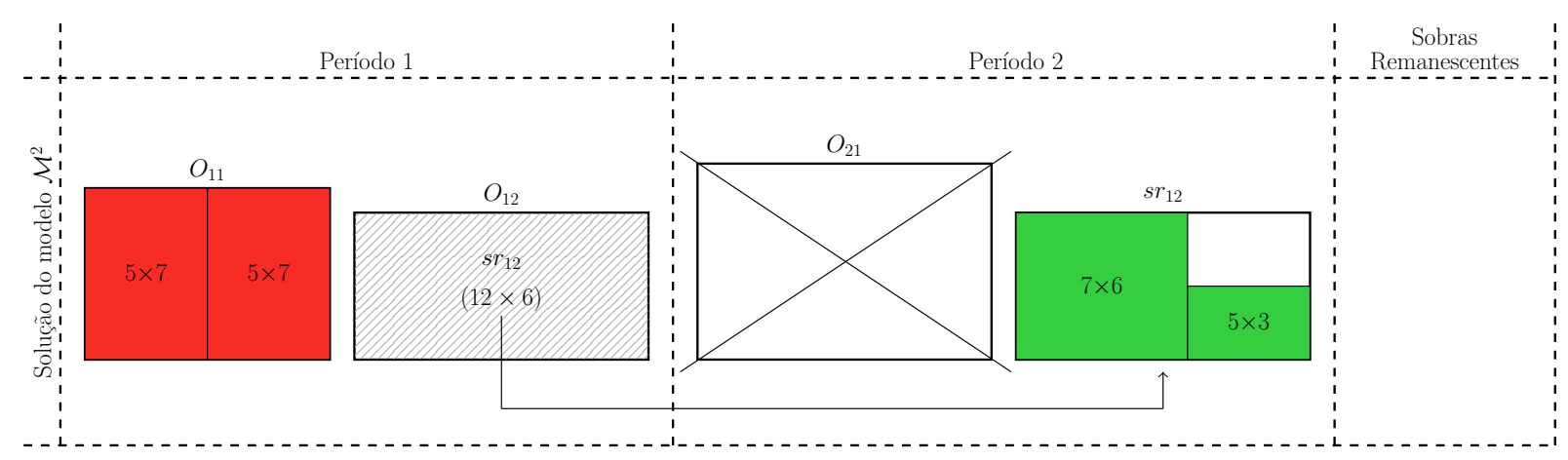

Figura 2.13: Solução da instância da Figura 2.11 usando o modelo $\mathcal{M}_{p, P}^{2}$. Neste modelo, não são permitidos cortes em objetos aos quais não sejam atribuídos itens. Note que a diferença entre essa solução e a solução encontrada pelo modelo $\mathcal{M}_{p, P}^{1}$ é o corte existente no objeto $O_{12}$, o que impede a geração de uma sobra aproveitável remanescente. A solução também possui custo dos objetos utilizados igual a 142, mas não possui sobras aproveitáveis remanescentes.

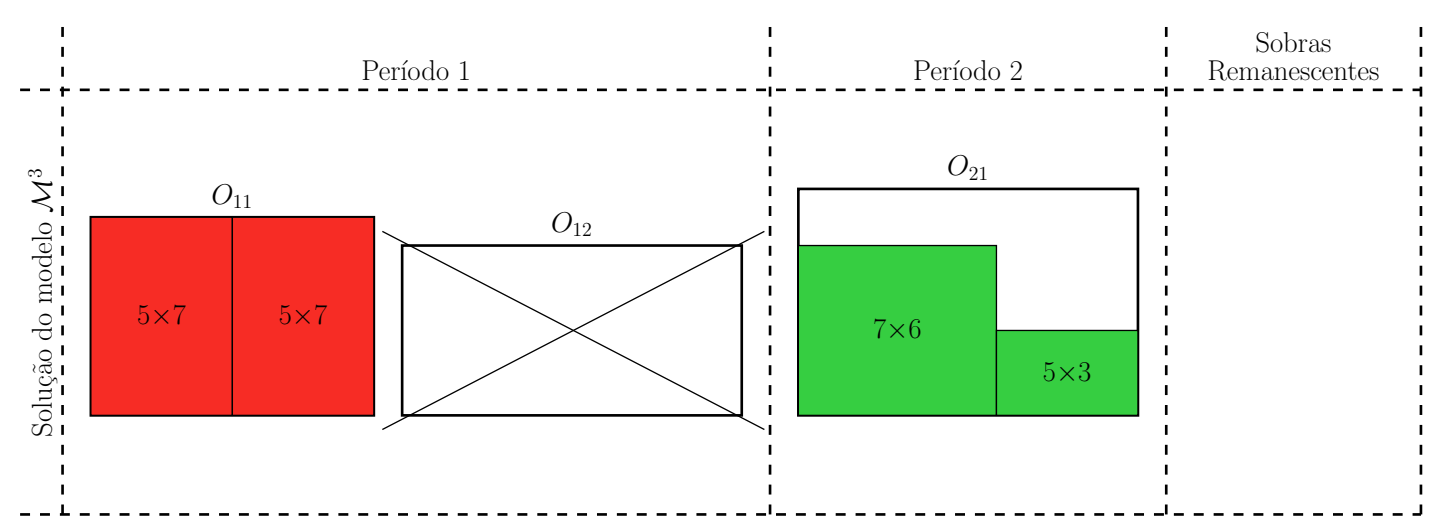

Figura 2.14: Solução da instância da Figura 2.11 usando o modelo $\mathcal{M}_{p, P}^{3}$. Este modelo impede que sejam cortados objetos aos quais não sejam atribuídos itens. Assim, no segundo período, é necessário utilizar o objeto $O_{21}$ para cortar os itens do período. Dessa forma, a solução tem custo igual a 166 e não possui sobras aproveitáveis.

\subsection{Experimentos computacionais}

Nesta seção, apresentamos alguns experimentos computacionais ilustrativos conduzidos para avaliar os resultados obtidos pelos modelos $\mathcal{M}_{p, P}^{1}, \mathcal{M}_{p, P}^{2}$ e $\mathcal{M}_{p, P}^{3}$, que resolvem as formulações MIP do PCBMPSA. Os modelos foram implementados em $\mathrm{C}++$ e compilados com GNU g++ versão 5.4 com a opção -03 ativada. A formulação matemática dos modelos foi resolvida usando o IBM ILOG CPLEX versão 12.6.3, sendo implementada via ILOG Concert Technology. Com relação aos parâmetros do CPLEX, definimos a variável EpAGap (referente a diferença absoluta entre a melhor solução inteira encontrada pelo CPLEX e o limite inferior dado pela sua relaxação linear) para 0.99999 (o valor padrão é $10^{-6}$ ), uma vez que a solução é inteira o CPLEX pode parar quando a diferença, em módulo, entre o limitante inferior e superior for menor que 1. Entretanto, o valor padrão da variável EpGap é $10^{-4}$ (o que significa que CPLEX irá parar quando encontrar uma solução inteira viável, cuja diferença relativa com o limitante inferior for menor que $0,01 \%$ ), o que pode fazer com que o CPLEX pare 
antes de encontrar a solução ótima do problema, por isso, definimos o valor dessa variável para 0 . O tempo de relógio, para resolução de uma instância pelo CPLEX, foi limitado em 2 horas (definimos o parâmetro TiLim como 7200) e pedimos para que o CPLEX armazene a árvore Branch \& Bound em disco caso ela exceda 12000MB (definimos NodeFileInd como 3 e WorkMem como 12000). Os experimentos foram realizados em uma máquina Intel Core i7-3770 3.40GHz, com 16 GB de memória RAM e com Sistema Operacional Linux.

A Tabela 2.1 apresenta os dados das instâncias usadas nos experimentos numéricos, dessa seção, para o problema de corte bidimensional multiperíodo com sobras aproveitáveis. Para cada instância, temos o número que identifica a instância, o número de períodos, a quantidade de tipos distintos de objetos para cada período e suas respectivas dimensões no formato $N(W \times H)[C]$, tal que $N$ referese à quantidade de objetos com dimensões $(W \times H)$ e $C$ corresponde ao custo por unidade de área do objeto daquele tipo. Em seguida, temos as informações dos itens: a quantidade de itens em cada período $(n)$, a quantidade de itens distintos $(\tilde{n})$, o número de itens do catálogo $(d)$ e suas respectivas dimensões no formato $N(W \times H)$, tal que $N$ refere-se a quantidade de itens com dimensões $(W \times H)$. Os $d$ itens do catálogo são os itens sublinhados. Onde $N$ é omitido significa que existe apenas uma unidade do objeto ou item e onde $C$ é omitido significa que o custo por unidade de área do objeto é 1. Além disso, em todas as instâncias consideramos $p=1$.

A Tabela 2.2 resume os resultados dos experimentos numéricos para as dez instâncias considerando $\xi=P-p$ e os modelos propostos para o PCBMPSA. Na tabela, a primeira coluna refere-se ao modelo utilizado; a segunda indica a instância de teste; as próximas três colunas estão associadas à solução encontrada: valor da função objetivo, custo dos objetos usados e o valor das sobras finais; as colunas restantes estão associadas ao desempenho do CPLEX: o gap gerado pelo CPLEX, em porcentagem, a quantidade de iterações MIP realizadas pelo CPLEX, a quantidade de nós da árvore Branch \& Bound e o tempo de execução, em segundos. As entradas marcadas com “-” indicam que a medida vale 0 .

Como podemos perceber, o CPLEX conseguiu encontrar soluções ótimas para a maioria das instâncias considerando os três modelos e em um tempo computacional aceitável. O CPLEX não foi capaz de resolver as instâncias 9 e 10 para nenhum dos três modelos, o mesmo acontece na instância 5 usando o $\mathcal{M}_{1, P}^{3}$. Entretanto, essas instâncias são consideradas de tamanho pequeno. Ao comparar os três modelos, note que o $\mathcal{M}_{1, P}^{1}$ possui as soluções com menor custo dos objetos utilizados, como esperado, uma vez que é o modelo mais flexível, em seguida temos o modelo $\mathcal{M}_{1, P}^{2}$ e depois o $\mathcal{M}_{1, P}^{3}$, sendo este último, o mais restritivo dos três. O gráfico da Figura 2.15 evidencia esse fato, mostrando o custo dos objetos utilizados por cada instância pelos três modelos. Podemos destacar a instância 3 cujo custo dos objetos, respectivamente para cada modelo, é 165, 174 e 290. As Figuras 2.20, 2.21 e 2.22 ilustram as soluções encontradas por cada um dos modelos. O modelo $\mathcal{M}_{1, P}^{1}$, ao permitir utilizar e cortar objetos sem atribuir itens, utilizou os dois objetos mais baratos necessários para atender a ordem de trabalho dos itens (Figura 2.20), o objeto $15 \times 5$ foi comprado e cortado no primeiro período, mas só foi utilizado no terceiro e quarto períodos. Já o modelo $\mathcal{M}_{p, P}^{2}$, por não permitir cortes em objetos não utilizados, teve que comprar o objeto $12 \times 7(12 \%$ mais caro que o objeto $15 \times 5$ utilizado pelo $\mathcal{M}_{1, P}^{1}$ ) para conseguir cortar os itens da ordem de trabalho (Figura 2.21). Ao não permitir a 
utilização de objetos sem itens, o modelo $\mathcal{M}_{1, P}^{3}$ precisou utilizar o objeto $20 \times 10$ para atender a ordem de trabalho dos itens do terceiro e quarto período. Assim, o modelo $\mathcal{M}_{1, P}^{3}$ obteve uma solução cuja função objetivo é cerca de $43 \%$ e $40 \%$ maior que a função objetivo encontra pelo modelo $\mathcal{M}_{1, P}^{1}$ e $\mathcal{M}_{1, P}^{2}$, respectivamente.

Tabela 2.1: Descrição das instâncias de testes.

\begin{tabular}{|c|c|c|c|c|c|c|c|}
\hline \multirow{2}{*}{ \#Inst. } & \multirow{2}{*}{$P$} & \multicolumn{2}{|r|}{ Objetos } & \multicolumn{4}{|r|}{ Itens } \\
\hline & & $m_{s}$ & $W_{s j} \times H_{s j}$ & $n_{s}$ & $\tilde{n}_{s}$ & $d$ & $w_{s i} \times h_{s i}$ \\
\hline \multirow{3}{*}{1} & \multirow{3}{*}{3} & 3 & $21 \times 17,19 \times 19,24 \times 13$ & 2 & 2 & \multirow{3}{*}{1} & $10 \times 11,9 \times 11$ \\
\hline & & 1 & $10 \times 16$ & 3 & 3 & & $7 \times 6,7 \times 5,7 \times 4$ \\
\hline & & 1 & $10 \times 12$ & 2 & 1 & & $2(\underline{6 \times 3})$ \\
\hline \multirow{4}{*}{2} & \multirow{4}{*}{4} & 2 & $14 \times 8,16 \times 6$ & 3 & 3 & \multirow{4}{*}{2} & $3 \times 7,6 \times 8,4 \times 8$ \\
\hline & & 1 & $15 \times 10$ & 3 & 2 & & $5 \times 3,2(2 \times 5)$ \\
\hline & & 1 & $20 \times 15$ & 2 & 2 & & $5 \times 3, \underline{3 \times 2}$ \\
\hline & & 1 & $15 \times 10$ & 2 & 1 & & $2(2 \times \overline{3})$ \\
\hline \multirow{4}{*}{3} & \multirow{4}{*}{4} & 2 & $15 \times 6,15 \times 5$ & 3 & 2 & \multirow{4}{*}{2} & $2(\underline{1 \times 6}), 10 \times 6$ \\
\hline & & 1 & $12 \times 7$ & 1 & 1 & & $3 \times 5$ \\
\hline & & 1 & $20 \times 10$ & 2 & 2 & & $5 \times 3,3 \times 2$ \\
\hline & & 1 & $20 \times 8$ & 6 & 4 & & $2(2 \times 3), 10 \times 1, \underline{2 \times 2}, 2(5 \times 2)$ \\
\hline \multirow{4}{*}{4} & \multirow{4}{*}{4} & 2 & $13 \times 8,12 \times 6$ & 5 & 5 & \multirow{4}{*}{2} & $1 \times 5,2 \times 5,1 \times 4,1 \times 3,3 \times 2$ \\
\hline & & 3 & $10 \times 8,12 \times 10,15 \times 10$ & 3 & 3 & & $3 \times 7,2 \times 3,2 \times 4$ \\
\hline & & 1 & $8 \times 4$ & 2 & 2 & & $10 \times 1, \underline{1 \times 3}$ \\
\hline & & 0 & & 3 & 3 & & $\underline{3 \times 1,3 \times 3,4 \times 4}$ \\
\hline \multirow{4}{*}{5} & \multirow{4}{*}{4} & 2 & $10 \times 4,13 \times 8$ & 4 & 3 & \multirow{4}{*}{1} & $2(1 \times 5), 2 \times 5,3 \times 5$ \\
\hline & & 2 & $10 \times 9,12 \times 9$ & 2 & 2 & & $5 \times 3,6 \times 3$ \\
\hline & & 3 & $10 \times 10,2(12 \times 9)$ & 3 & 3 & & $5 \times 3,6 \times 2,3 \times 3$ \\
\hline & & 0 & & 3 & 3 & & $\underline{1 \times 2,5 \times 4,4 \times 2}$ \\
\hline \multirow{4}{*}{6} & \multirow{4}{*}{4} & 2 & $22 \times 17,14 \times 30$ & 5 & 2 & \multirow{4}{*}{4} & $3(2 \times 11), 2(5 \times 5)$ \\
\hline & & 2 & $17 \times 29,24 \times 10$ & 2 & 1 & & $2(\overline{4 \times 10})$ \\
\hline & & 2 & $18 \times 19,26 \times 22$ & 3 & 1 & & $3(5 \times 4)$ \\
\hline & & 3 & $24 \times 12,15 \times 18,17 \times 13$ & 8 & 4 & & $4(\underline{3 \times 3}), \underline{4 \times 2}, 2(\underline{7 \times 1}), 11 \times 1$ \\
\hline \multirow{4}{*}{7} & \multirow{4}{*}{4} & 2 & $(10 \times 12)[2], 12 \times 10$ & 3 & 3 & \multirow{4}{*}{1} & $5 \times 4,8 \times 2, \underline{2 \times 2}$ \\
\hline & & 1 & $17 \times 15$ & 1 & 1 & & $3 \times 7$ \\
\hline & & 1 & $17 \times 15$ & 1 & 1 & & $8 \times 4$ \\
\hline & & 1 & $17 \times 15$ & 1 & 1 & & $4 \times 9$ \\
\hline & & 2 & $10 \times 12,(12 \times 10)[2]$ & 3 & 3 & & $5 \times 4,8 \times 2, \underline{2 \times 2}$ \\
\hline 8 & 4 & 1 & $17 \times 15$ & 1 & 1 & 1 & $3 \times 7$ \\
\hline$\gamma$ & 4 & 1 & $17 \times 15$ & 1 & 1 & 1 & $8 \times 4$ \\
\hline & & 1 & $17 \times 15$ & 1 & 1 & & $4 \times 9$ \\
\hline & & 3 & $30 \times 20,2(10 \times 10)[3]$ & 6 & 6 & & $3 \times 7,8 \times 2,10 \times 1,5 \times 4,2 \times 9,2 \times 2$ \\
\hline 9 & 4 & 3 & $(30 \times 20)[3], 2(10 \times 10)[3]$ & 6 & 6 & 2 & $5 \times 3,9 \times 3,6 \times 1,3 \times 8,4 \times 1,7 \overline{\times 3}$ \\
\hline 9 & 4 & 0 & & 4 & 4 & $Z$ & $3 \times 2,7 \times 2,4 \times 5, \underline{4 \times 1}$ \\
\hline & & 0 & & 4 & 4 & & $8 \times 4,4 \times 2,3 \times 7, \overline{6 \times 2}$ \\
\hline & & 2 & $14 \times 21,19 \times 19$ & 7 & 3 & & $2(11 \times 3), 3(2 \times 11), 2(5 \times 5)$ \\
\hline 10 & 4 & 1 & $27 \times 23$ & 9 & 4 & 1 & $9 \times 7,4(9 \times 6), 2(5 \times 3), 2(5 \times 4)$ \\
\hline 10 & 4 & 1 & $20 \times 15$ & 9 & 2 & 1 & $5(3 \times 2), 4(3 \times 1)$ \\
\hline & & 1 & $17 \times 17$ & 7 & 2 & & $4(3 \times 4), 3(\underline{2 \times 1})$ \\
\hline
\end{tabular}


Tabela 2.2: Resultado numérico para as instâncias do PCBMPSA

\begin{tabular}{|c|c|c|c|c|c|c|c|c|}
\hline \multirow{2}{*}{ Modelo } & \multirow{2}{*}{ \#Inst. } & \multicolumn{3}{|c|}{ Dados da Solução } & \multicolumn{4}{|c|}{ Medidas de desempenho } \\
\hline & & $\begin{array}{l}\text { Função } \\
\text { Objetivo }\end{array}$ & $\begin{array}{c}\text { Custo } \\
\text { Objetos }\end{array}$ & $\begin{array}{l}\text { Valor } \\
\text { Sobras }\end{array}$ & $\operatorname{Gap}(\%)$ & $\begin{array}{c}\text { Iterações } \\
\text { MIP }\end{array}$ & Nós B\&B & Tempo(s) \\
\hline \multirow{10}{*}{$\mathcal{M}_{1, P}^{1}$} & 1 & 624.810 & 477 & 60 & - & 74.620 & 4.799 & 1,53 \\
\hline & 2 & 168.040 & 208 & 24 & - & 58.208 .431 & 7.477 .859 & 855,39 \\
\hline & 3 & 100.485 & 165 & 0 & - & 197.140 & 33.541 & 3,73 \\
\hline & 4 & 58.032 & 104 & 0 & - & 251.728 & 21.215 & 5,21 \\
\hline & 5 & 94.742 & 144 & 10 & - & 2.735 .823 & 357.559 & 48,95 \\
\hline & 6 & 1.204 .236 & 374 & 44 & - & 119.466 .091 & 20.072 .179 & $2.037,05$ \\
\hline & 7 & 404.802 & 360 & 198 & - & 11.407 .352 & 3.744 .062 & 280,56 \\
\hline & 8 & 404.825 & 360 & 175 & - & 79.579 .887 & 23.234 .603 & $1.714,96$ \\
\hline & 9 & 2.159 .727 & 600 & 273 & 0,04 & 145.984 .495 & 9.656 .785 & $7.200,00$ \\
\hline & 10 & 1.889 .489 & 799 & 146 & 0,30 & 261.805.109 & 5.666 .871 & $7.200,00$ \\
\hline \multirow{10}{*}{$\mathcal{M}_{1, P}^{2}$} & 1 & 624.810 & 477 & 60 & - & 54.451 & 4.304 & 2,07 \\
\hline & 2 & 168.064 & 208 & 0 & - & 192.659 & 16.217 & 4,14 \\
\hline & 3 & 105.954 & 174 & 12 & - & 704.647 & 35.003 & 8,48 \\
\hline & 4 & 58.032 & 104 & 0 & - & 99.277 & 5.815 & 3,61 \\
\hline & 5 & 94.748 & 144 & 4 & - & 62.113 .627 & 15.738 .108 & $1.135,66$ \\
\hline & 6 & 1.204 .236 & 374 & 44 & - & 102.307 .810 & 19.865 .774 & $1.914,57$ \\
\hline & 7 & 404.802 & 360 & 198 & - & 2.754 .213 & 780.476 & 57,11 \\
\hline & 8 & 404.825 & 360 & 175 & - & 30.073 .116 & 11.633 .045 & 687,08 \\
\hline & 9 & 2.159 .727 & 600 & 273 & 0,03 & 150.928 .847 & 7.915 .327 & $7.200,00$ \\
\hline & 10 & 1.889 .468 & 799 & 167 & 0,28 & 266.162 .428 & 6.437 .074 & $7.200,00$ \\
\hline \multirow{10}{*}{$\mathcal{M}_{1, P}^{3}$} & 1 & 624.810 & 477 & 60 & - & 39.446 & 2.993 & 1,25 \\
\hline & 2 & 211.619 & 262 & 77 & - & 7.905 .660 & 1.222 .646 & 110,71 \\
\hline & 3 & 176.480 & 290 & 130 & - & 604.200 & 132.509 & 13,16 \\
\hline & 4 & 58.032 & 104 & 0 & - & 109.796 & 3.909 & 3,70 \\
\hline & 5 & 127.592 & 194 & 60 & 0,02 & 338.484 .657 & 62.706 .967 & $7.200,00$ \\
\hline & 6 & 1.204 .236 & 374 & 44 & - & 43.775 .371 & 9.802 .642 & 996,32 \\
\hline & 7 & 404.810 & 360 & 190 & - & 5.592 .986 & 1.769 .842 & 104,98 \\
\hline & 8 & 404.825 & 360 & 175 & - & 55.508 .518 & 18.890 .931 & $1.244,80$ \\
\hline & 9 & 2.159 .727 & 600 & 273 & 0,03 & 155.372 .221 & 9.797 .712 & $7.200,00$ \\
\hline & 10 & 1.889 .498 & 799 & 137 & 0,24 & 265.661 .151 & 4.575 .071 & $7.200,00$ \\
\hline
\end{tabular}

Observe que nas instâncias 2 e 5 os modelos $\mathcal{M}_{1, P}^{1}$ e $\mathcal{M}_{1, P}^{2}$ encontram soluções de mesmo custo dos objetos, mas com valor das sobras finais diferentes. Nessas instâncias, o modelo $\mathcal{M}_{1, P}^{3}$, por ser mais restritivo, obteve soluções com custos dos objetos maiores. As Figuras 2.17, 2.18 e 2.19 mostram as soluções obtidas por cada modelo para a instância 2 e as soluções da instância 5 são ilustradas pelas Figuras 2.23, 2.24 e 2.25. Na instância 7, os três modelos retornaram soluções com o mesmo custo dos objetos, veja as Figuras 2.26 e 2.27. Entretanto, o modelo $\mathcal{M}_{1, P}^{3}$, por não permitir a utilização de objetos sem itens, precisou atribuir um item (o menor para maximizar a sobra aproveitável) do primeiro período ao objeto $10 \times 12$ (Figura 2.27a) de forma que, agora, o objeto possa ser utilizado, com o objetivo de encontrar uma solução de custo menor. Caso isso não fosse feito, um objeto mais caro seria necessário para cortar os itens de ordens de trabalho futuras. 


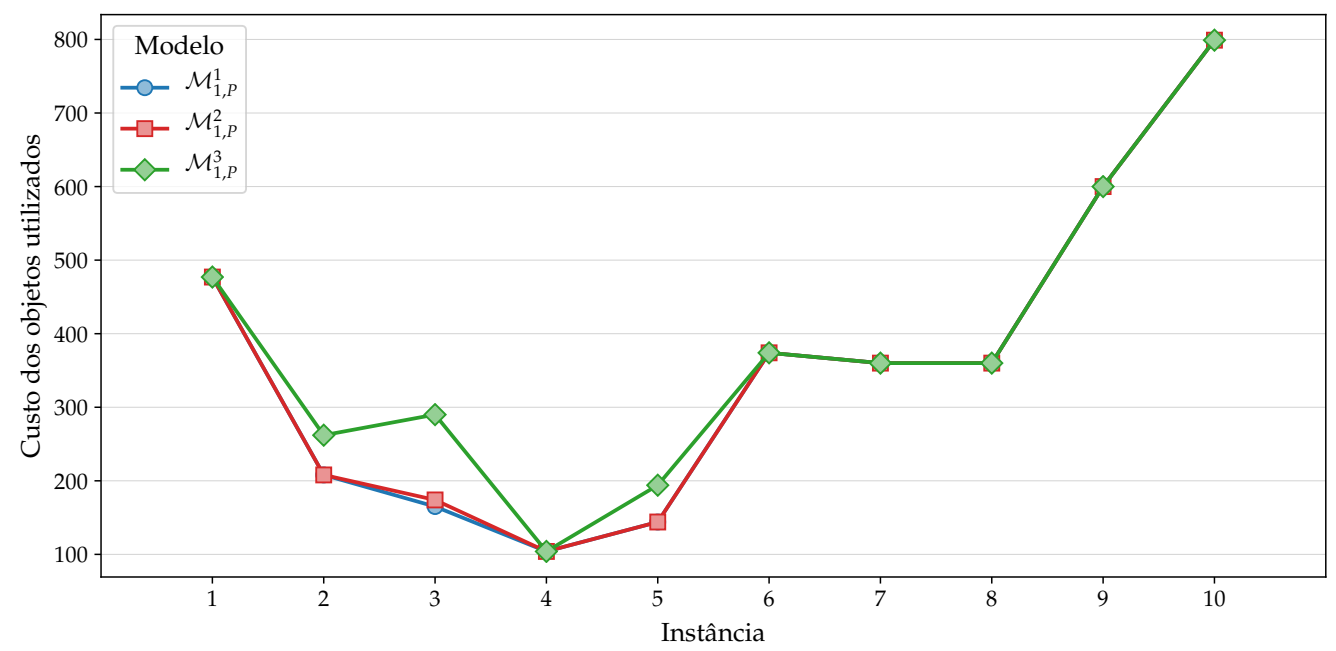

Figura 2.15: Gráfico do custo dos objetos, para cada instância, obtido em cada um dos modelos. Note que os modelos $\mathcal{M}_{1, P}^{1}$ e $\mathcal{M}_{1, P}^{2}$ encontram praticamente todas as soluções com mesmo custo, exceto na instância 3. Contudo, o modelo $\mathcal{M}_{1, P}^{3}$ encontrou três soluções com custo maior.

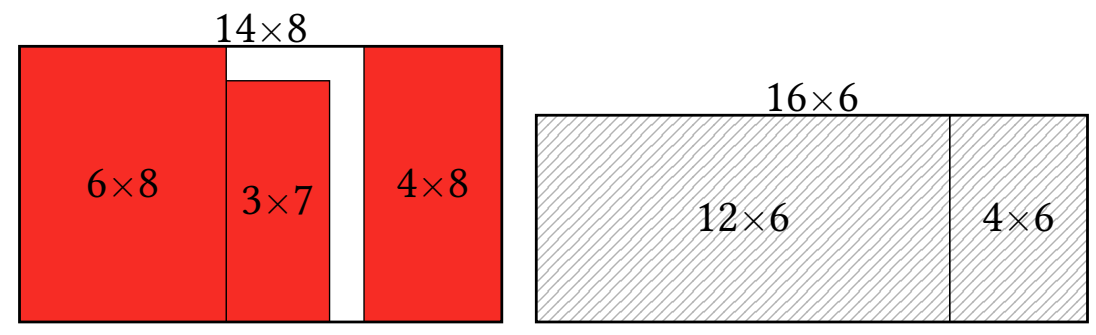

(a) Período 1

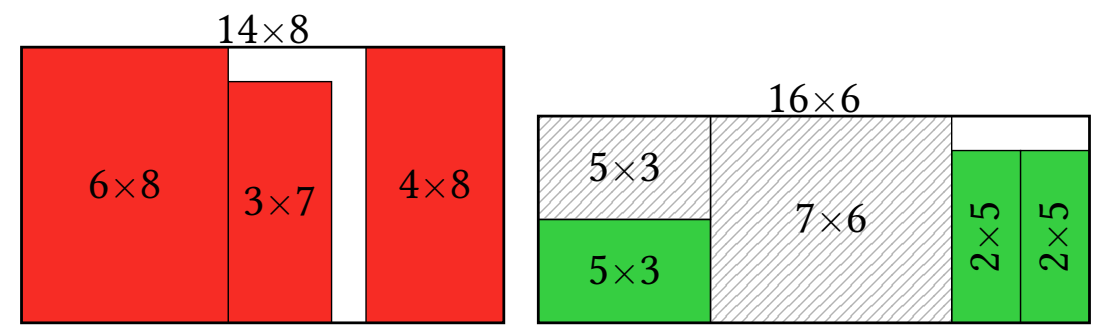

(b) Período 2

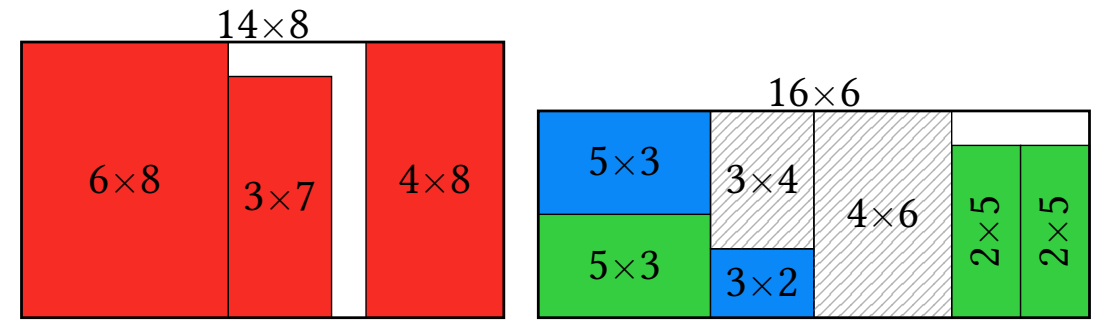

(c) Período 3

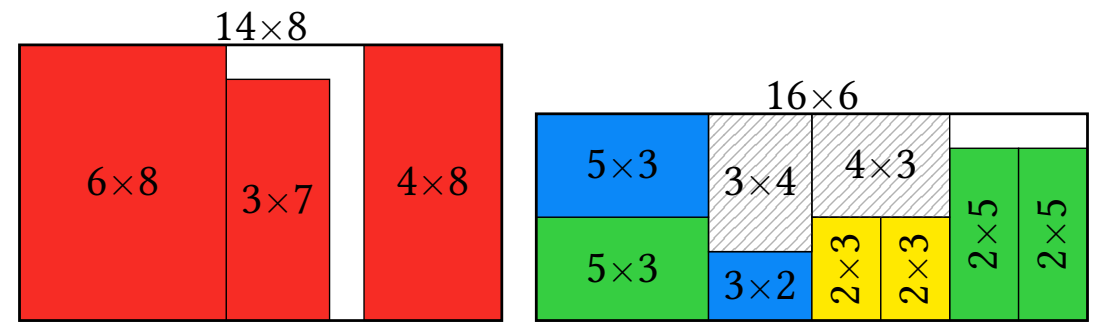

(d) Período 4

Figura 2.17: Ilustrações da solução obtida pelo modelo $\mathcal{M}_{1, P}^{1}$ para a Instância 2, considerando a legenda mostrada na Figura 2.16. 


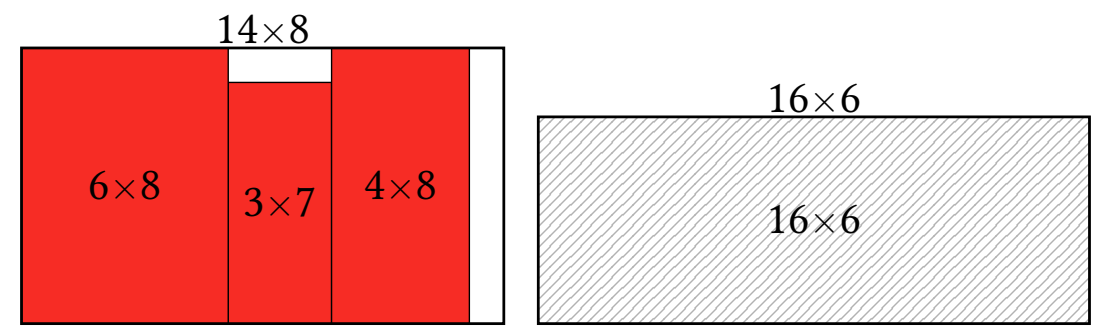

(a) Período 1

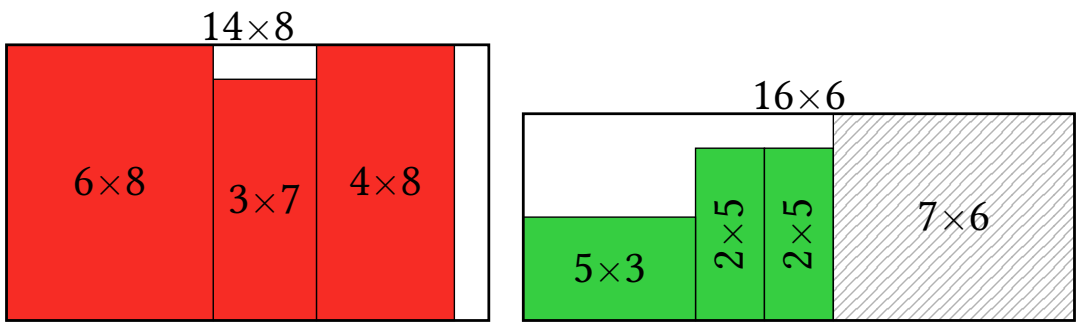

(b) Período 2

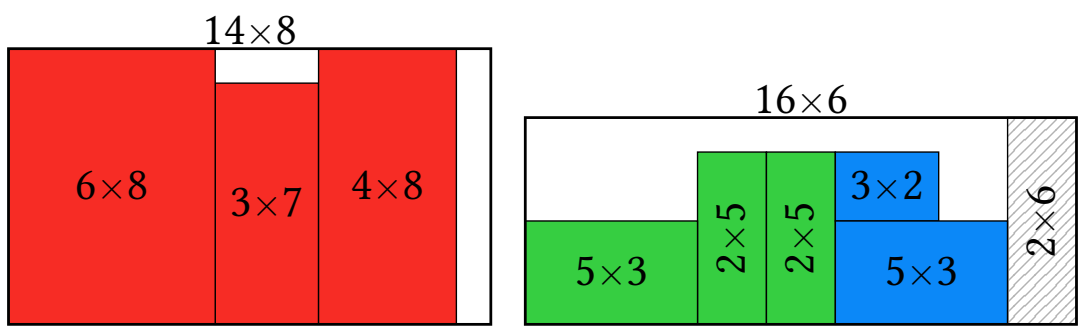

(c) Período 3

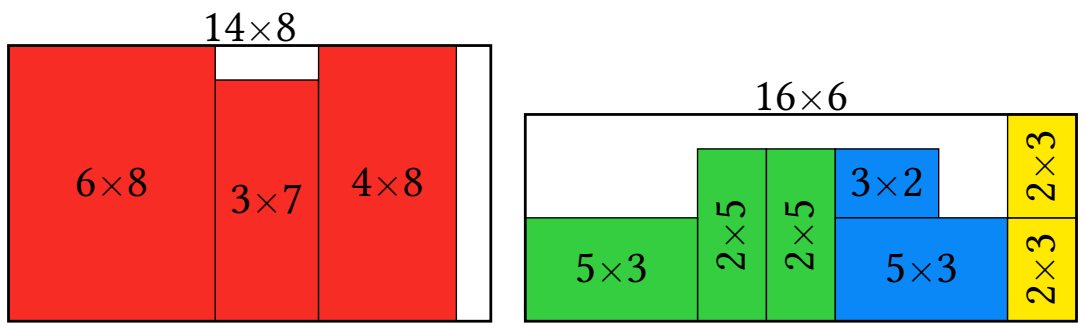

(d) Período 4

Figura 2.18: Ilustrações da solução obtida pelo modelo $\mathcal{M}_{1, P}^{2}$ para a Instância 2, considerando a legenda mostrada na Figura 2.16. 
$14 \times 8$

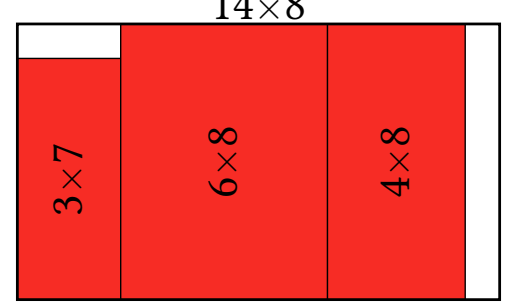

(a) Período 1

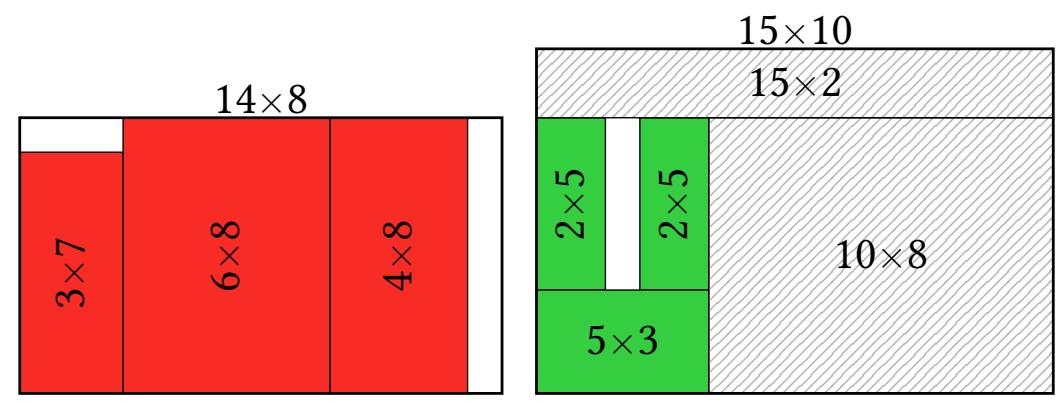

(b) Período 2

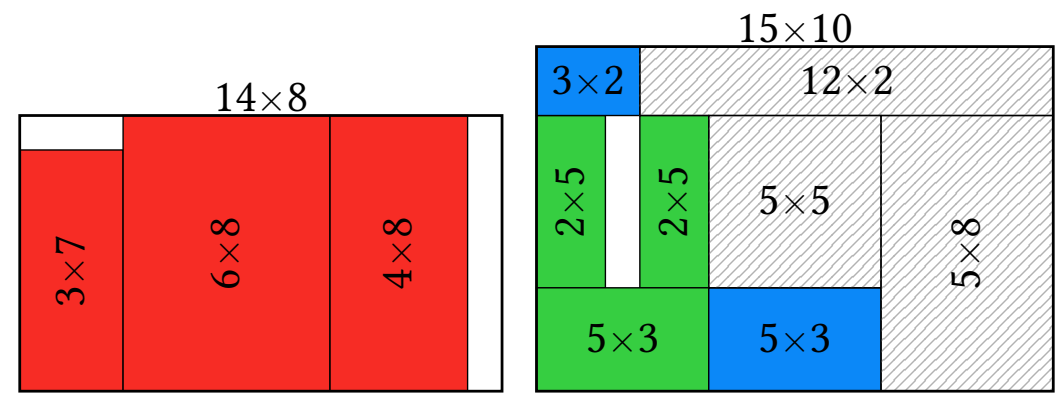

(c) Período 3

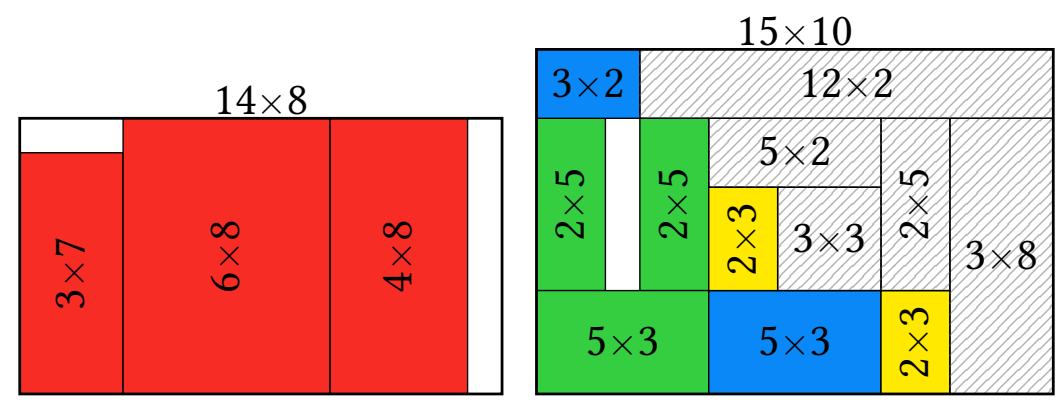

(d) Período 4

Figura 2.19: Ilustrações da solução obtida pelo modelo $\mathcal{M}_{1, P}^{3}$ para a Instância 2, considerando a legenda mostrada na Figura 2.16. 
$15 \times 6$

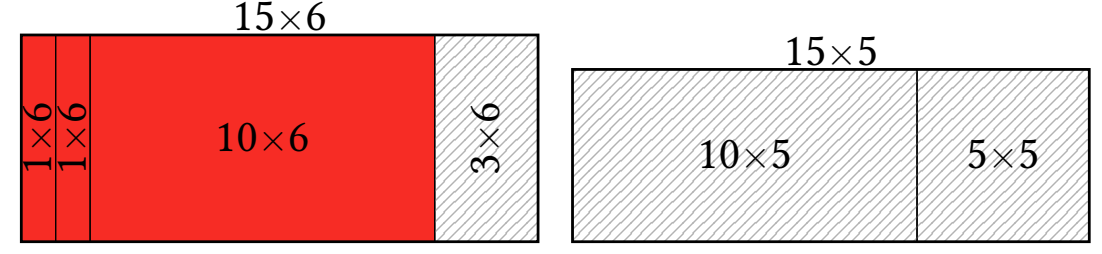

(a) Período 1

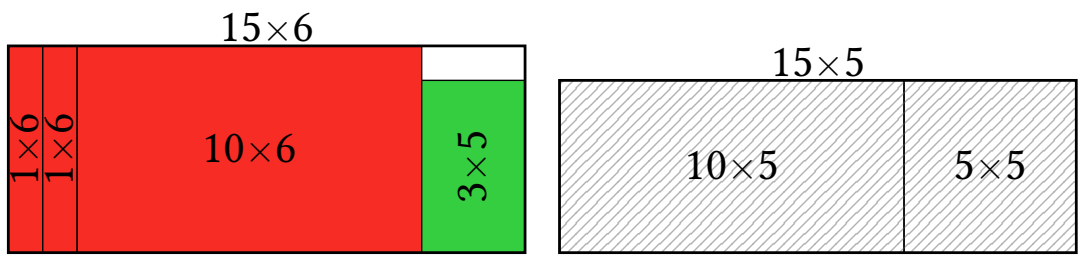

(b) Período 2

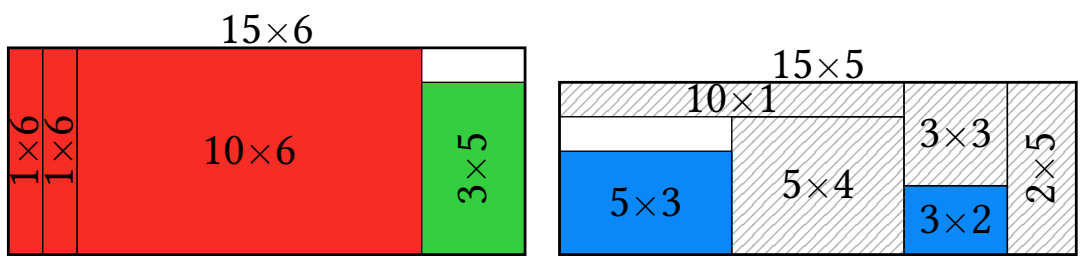

(c) Período 3

$15 \times 6$

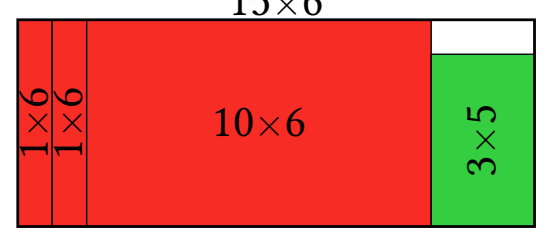

\begin{tabular}{|c|c|c|c|}
\hline \multicolumn{4}{|c|}{$15 \times 5$} \\
\hline & $5 \times 2$ & $\stackrel{x}{N}$ & \\
\hline $5 \times$ & $5 \times 2$ & $3 \times 2$ & N \\
\hline
\end{tabular}

(d) Período 4

Figura 2.20: Ilustrações da solução obtida pelo modelo $\mathcal{M}_{1, P}^{1}$ para a Instância 3, considerando a legenda mostrada na Figura 2.16. 
$15 \times 6$

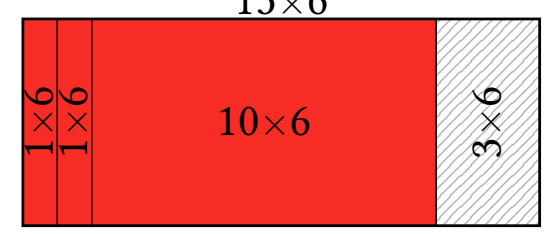

(a) Período 1

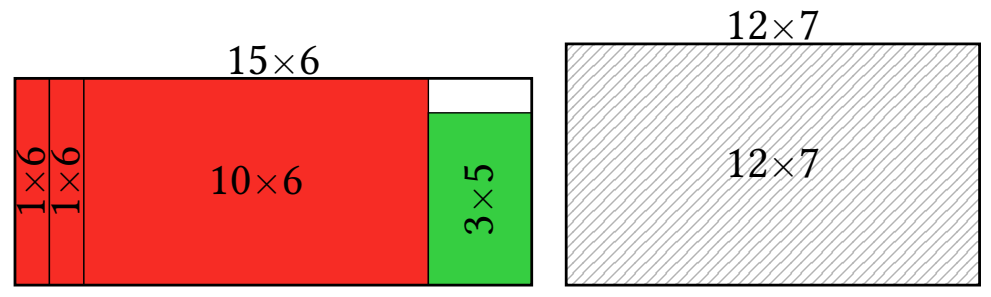

(b) Período 2

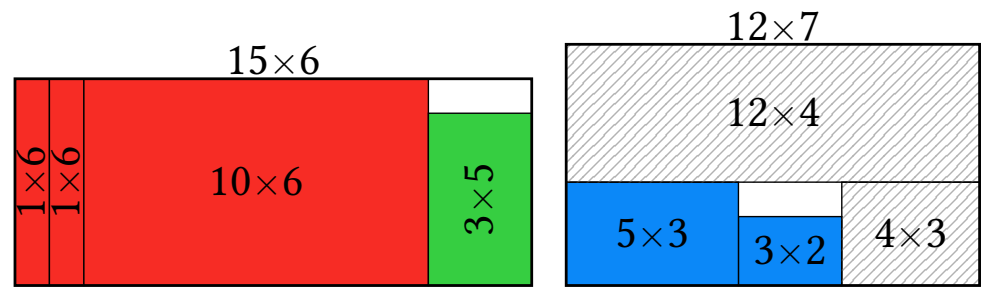

(c) Período 3

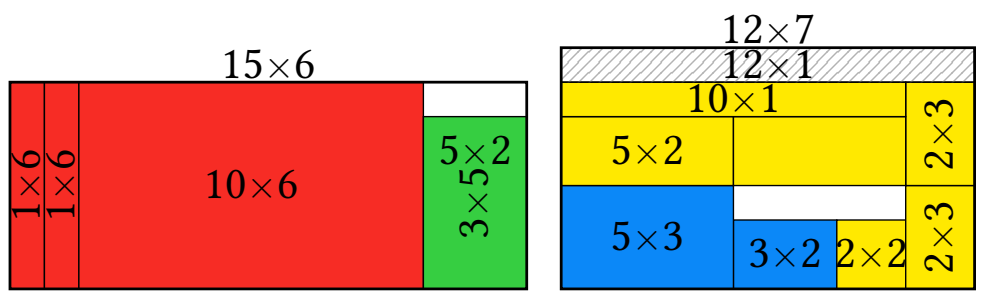

(d) Período 4

Figura 2.21: Ilustrações da solução obtida pelo modelo $\mathcal{M}_{1, P}^{2}$ para a Instância 3, considerando a legenda mostrada na Figura 2.16. 


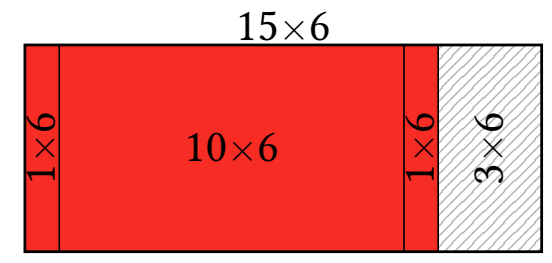

(a) Período 1

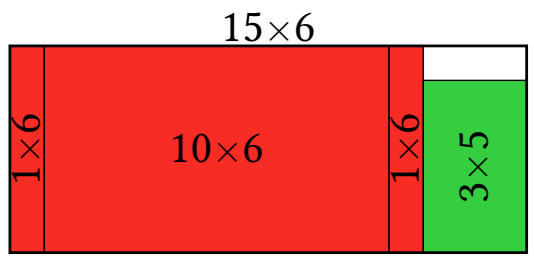

(b) Período 2

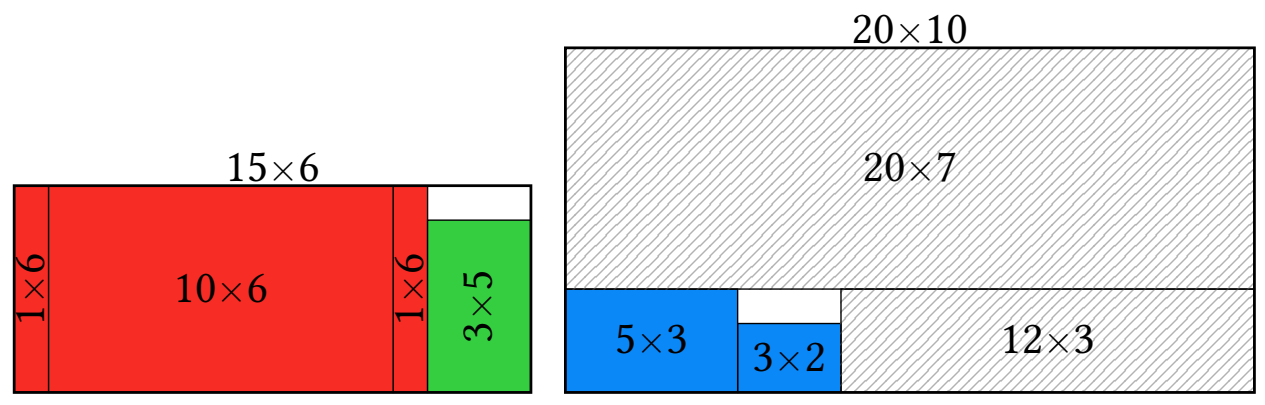

(c) Período 3

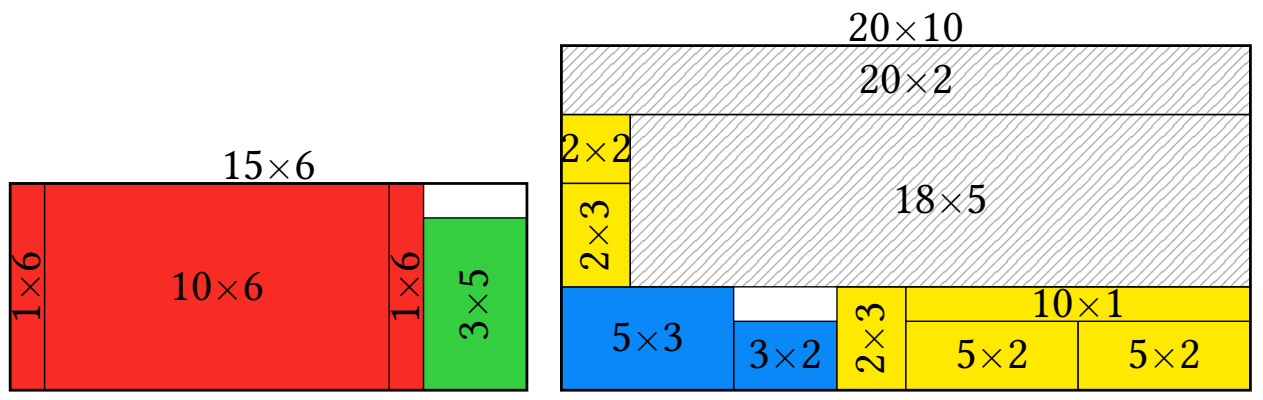

(d) Período 4

Figura 2.22: Ilustrações da solução obtida pelo modelo $\mathcal{M}_{1, P}^{3}$ para a Instância 3 , considerando a legenda mostrada na Figura 2.16. 


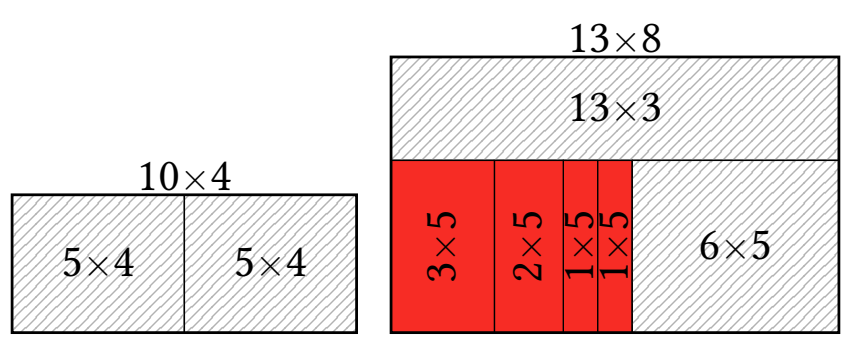

(a) Período 1

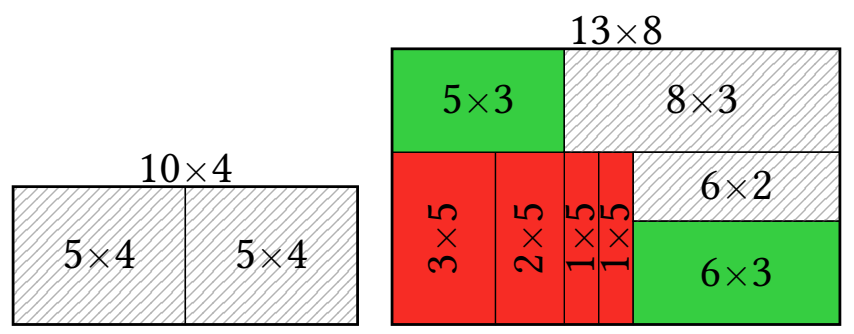

(b) Período 2

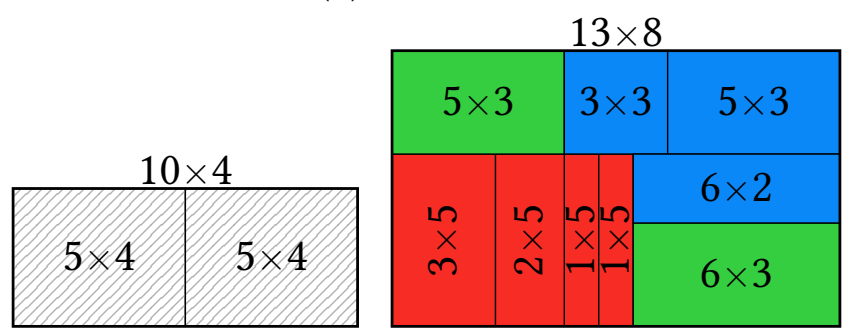

(c) Período 3

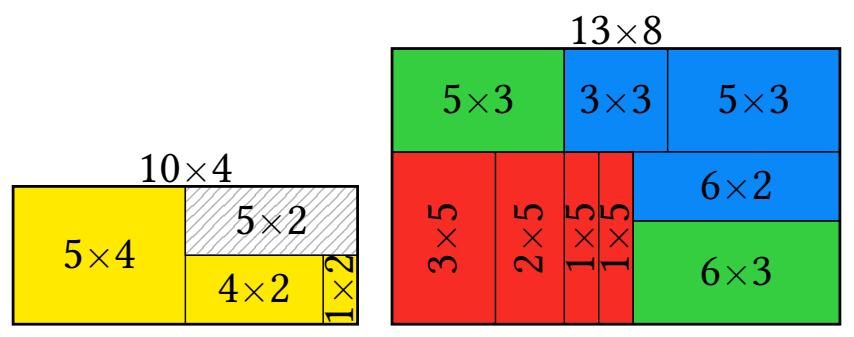

(d) Período 4

Figura 2.23: Ilustrações da solução obtida pelo modelo $\mathcal{M}_{1, P}^{1}$ para a Instância 5 , considerando a legenda mostrada na Figura 2.16. 


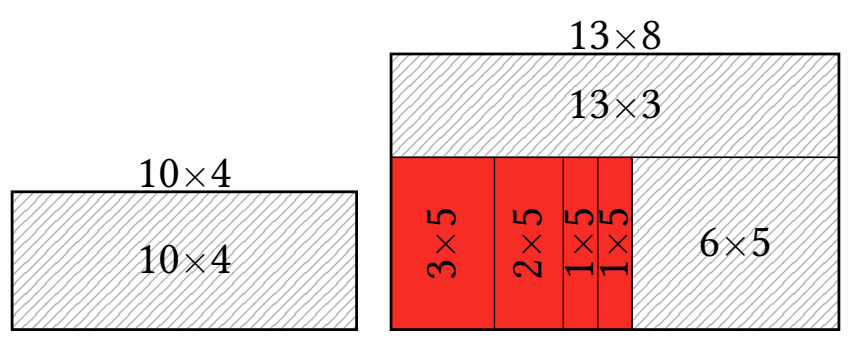

(a) Período 1

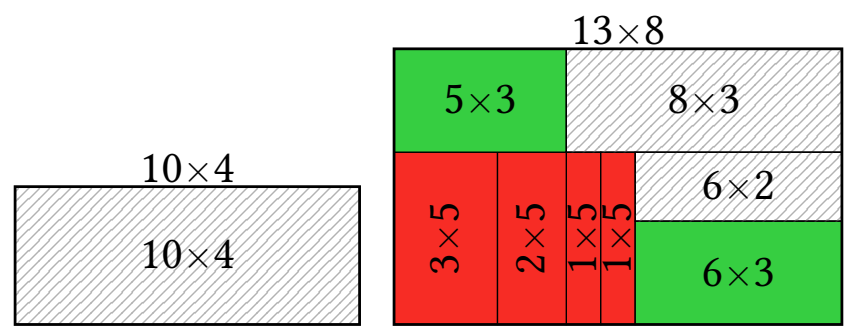

(b) Período 2

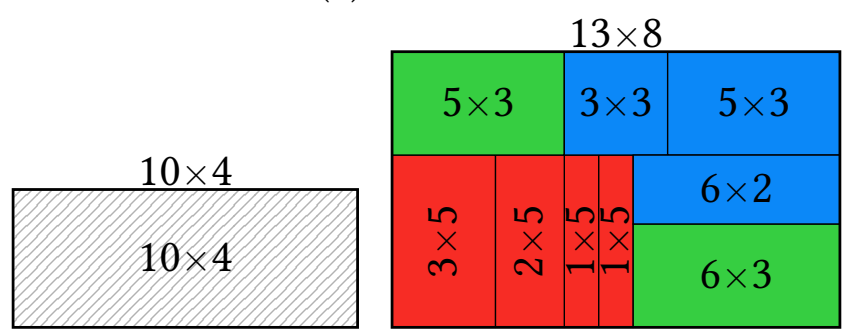

(c) Período 3

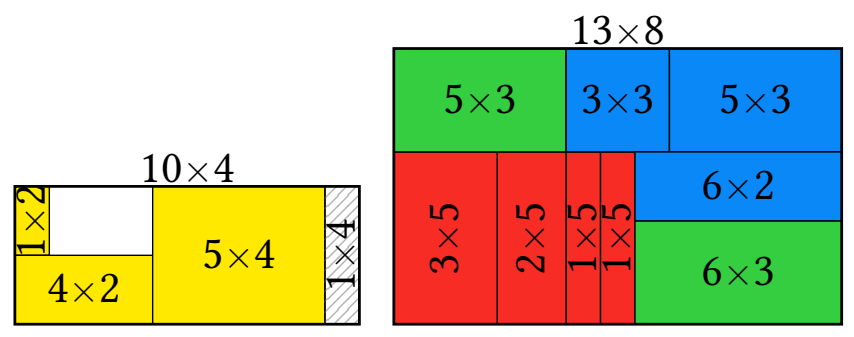

(d) Período 4

Figura 2.24: Ilustrações da solução obtida pelo modelo $\mathcal{M}_{1, P}^{2}$ para a Instância 5 , considerando a legenda mostrada na Figura 2.16. 


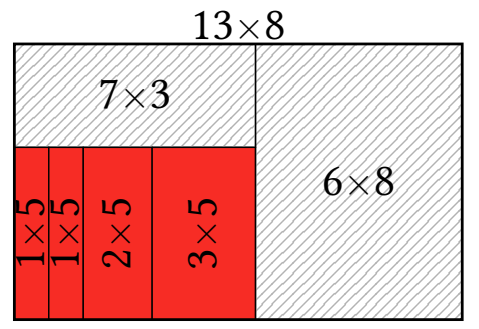

(a) Período 1

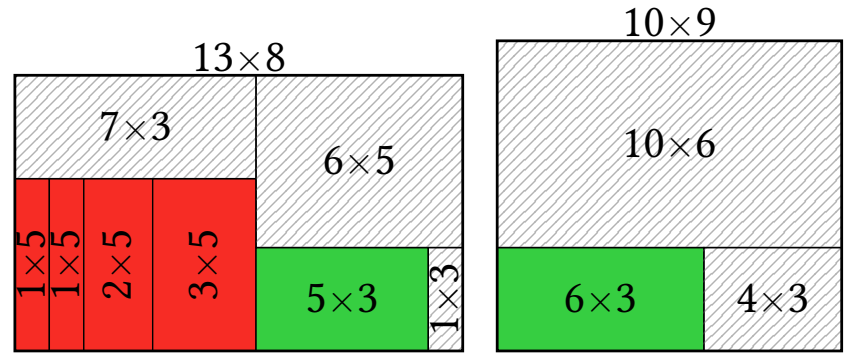

(b) Período 2

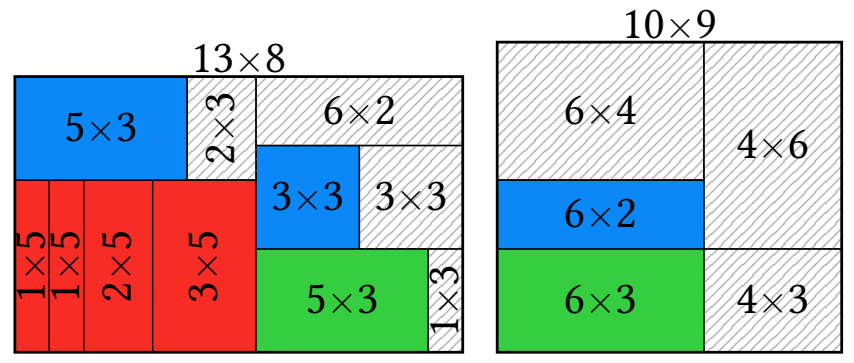

(c) Período 3

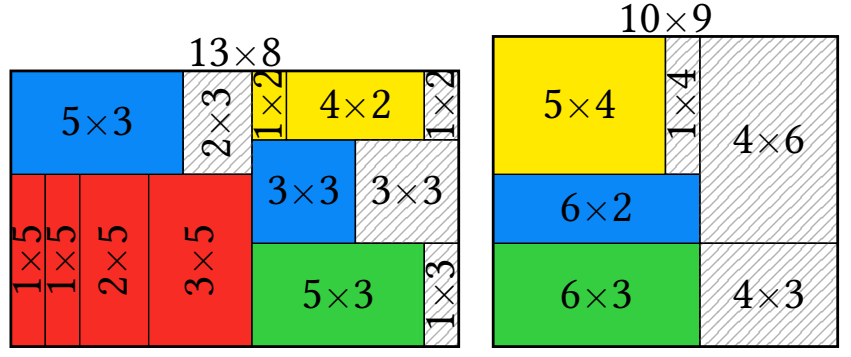

(d) Período 4

Figura 2.25: Ilustrações da solução obtida pelo modelo $\mathcal{M}_{1, P}^{3}$ para a Instância 5 , considerando a legenda mostrada na Figura 2.16. 


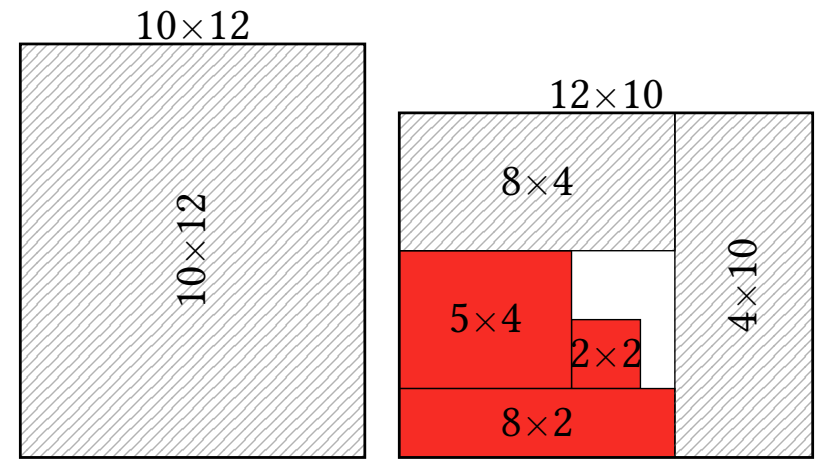

(a) Período 1

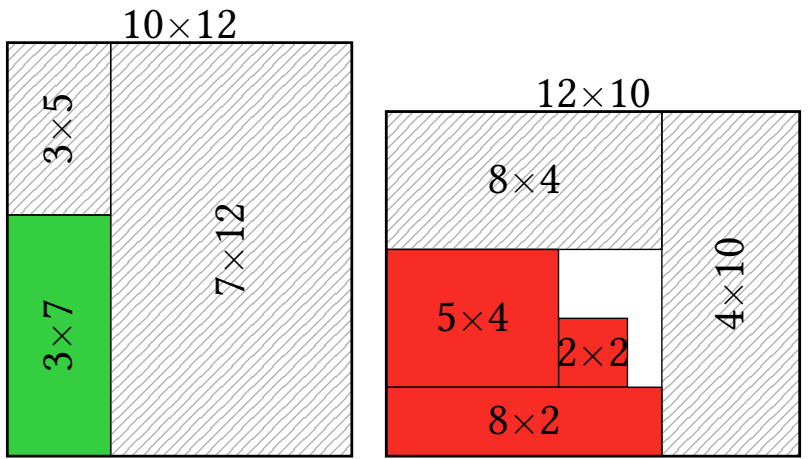

(b) Período 2

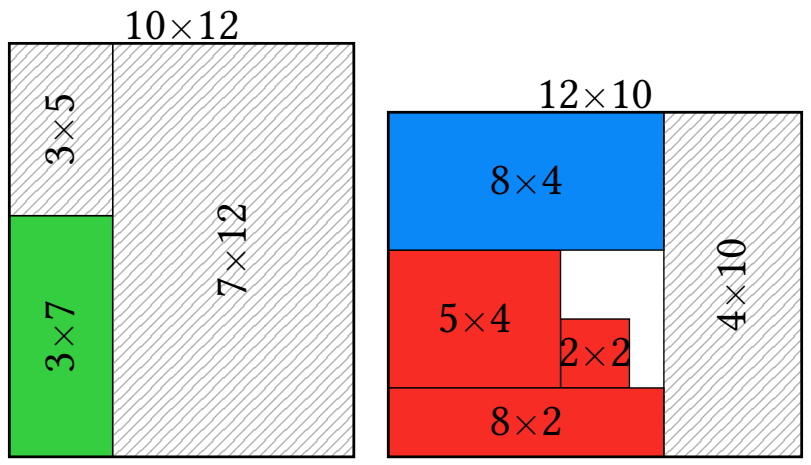

(c) Período 3

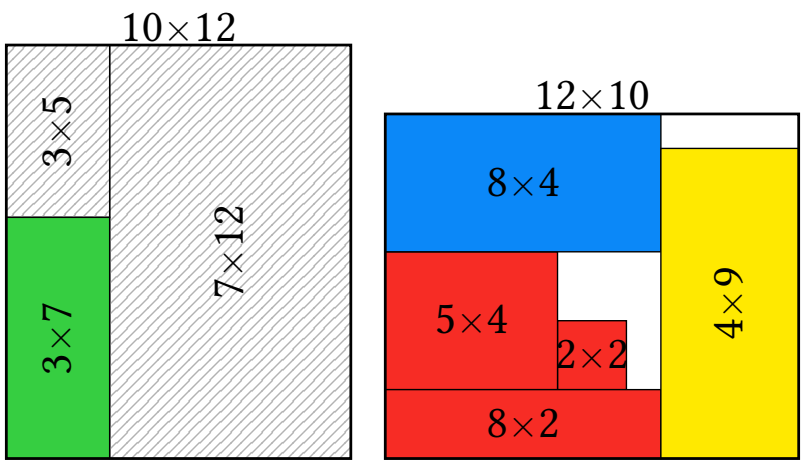

(d) Período 4

Figura 2.26: Ilustrações da solução obtida pelo modelo $\mathcal{M}_{1, P}^{1}$ e $\mathcal{M}_{1, P}^{2}$ para a Instância 7, considerando a legenda mostrada na Figura 2.16. 


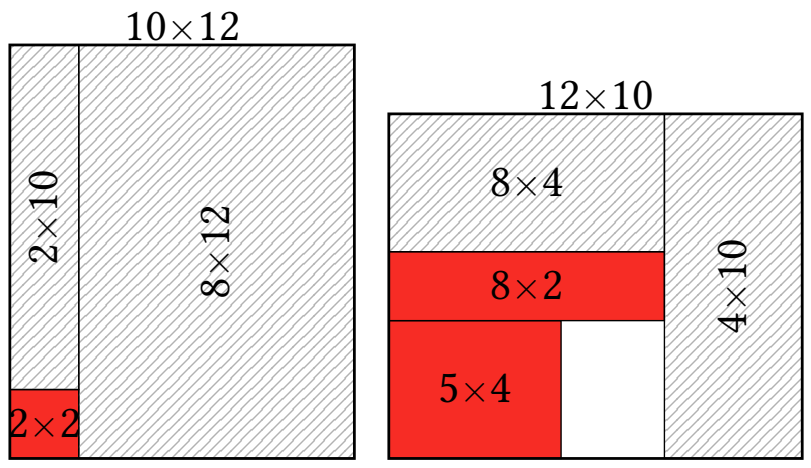

(a) Período 1

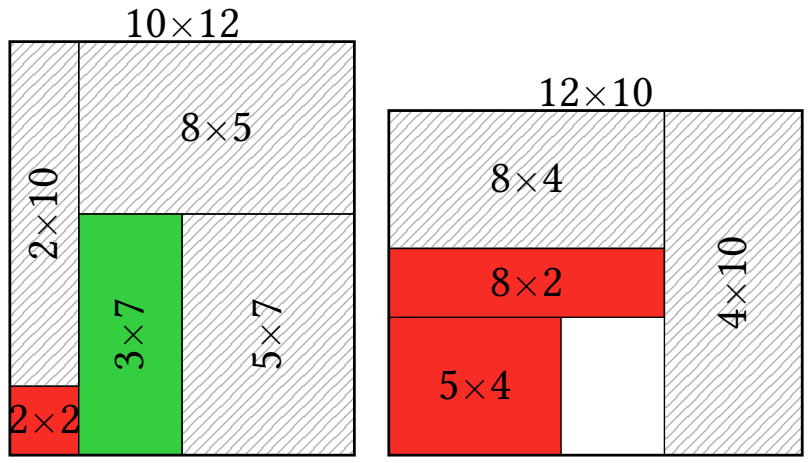

(b) Período 2

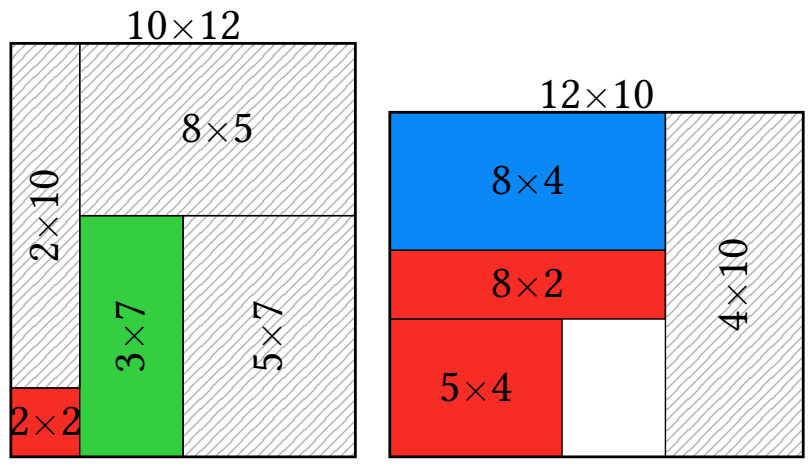

(c) Período 3

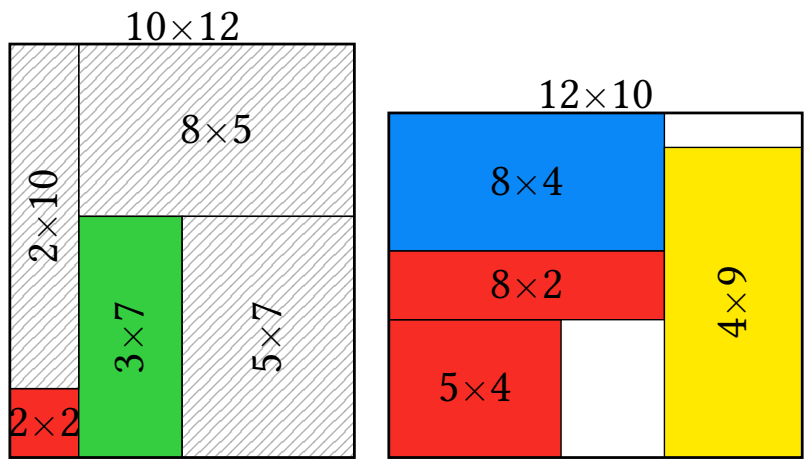

(d) Período 4

Figura 2.27: Ilustrações da solução obtida pelo modelo $\mathcal{M}_{1, P}^{3}$ para a Instância 2, considerando a legenda mostrada na Figura 2.16. 
Com o objetivo de conseguir resolver instâncias maiores, podemos limitar o número de períodos $\xi$ que uma sobra pode estar disponível para a utilização. Inicialmente, vamos resolver os modelos $\mathcal{M}_{1, P}^{1}, \mathcal{M}_{1, P}^{2}$ e $\mathcal{M}_{1, P}^{3}$ considerando, novamente, as instâncias mostradas na Tabela 2.1, mas agora com diferentes valores de $\xi$. A Tabela 2.3 apresenta os resultados dos três modelos para essas instâncias considerando $\xi \in\{1,2,3\}$, sendo que $\xi=3$ equivale aos resultados apresentados na Tabela 2.2.

$\mathrm{Na}$ Tabela 2.3, a primeira coluna refere-se ao modelo usado; a segunda indica a instância de teste; as próximas cinco colunas estão associadas à solução encontrada por cada modelo para $\xi=1$ : valor da função objetivo, custo dos objetos utilizados, valor das sobras finais, gap gerado pelo CPLEX, em porcentagem, e tempo de execução, em segundos; as próximas cinco colunas estão associadas à solução para $\xi=2$; e as cinco últimas estão associadas à solução para $\xi=3$. As entradas marcadas com "-" indicam que a medida vale 0 .

Como podemos notar, na maioria dos casos, quanto menor o valor de $\xi$ mais rápido o solver consegue encontrar a solução ótima da instância, independente do modelo. No entanto, como esperado, diminuindo $\xi$ também diminuímos a qualidade das soluções (veja os gráficos da Figura 2.28). Por exemplo, no $\mathcal{M}_{1, P}^{1}$, a função objetivo da instância 2 vale $332.758,221.619,168.040$, para $\xi=1, \xi=2$ e $\xi=3$, respectivamente. Note que a instância 9 é inviável quando $\xi=1$. Isso porque as sobras dos objetos de entrada do segundo período podem ser usadas apenas no terceiro período (que não possui objetos e, portanto, não disponibilizará sobras para serem utilizadas no quarto período), como no quarto período não há objetos de entrada e existem itens na ordem de trabalho, a instância torna-se inviável.

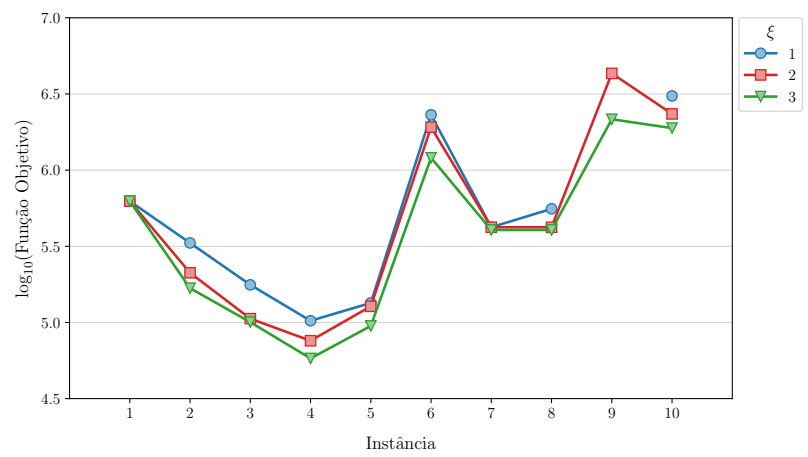

(a) $\mathcal{M}_{1, P}^{1}$

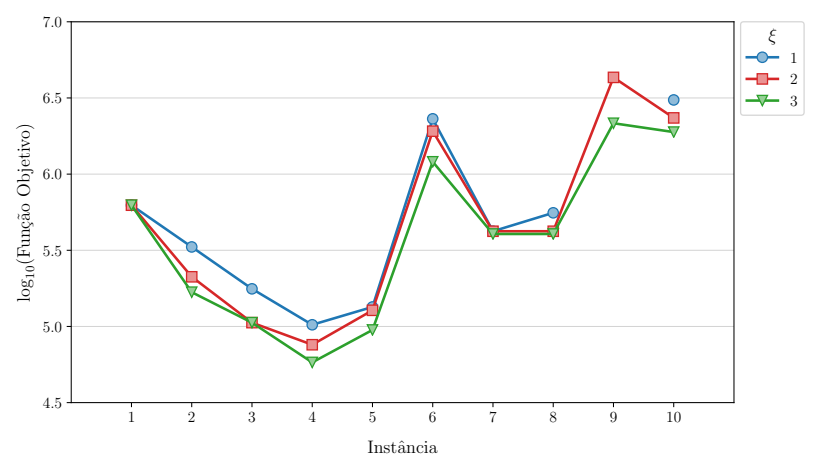

(b) $\mathcal{M}_{1, P}^{2}$

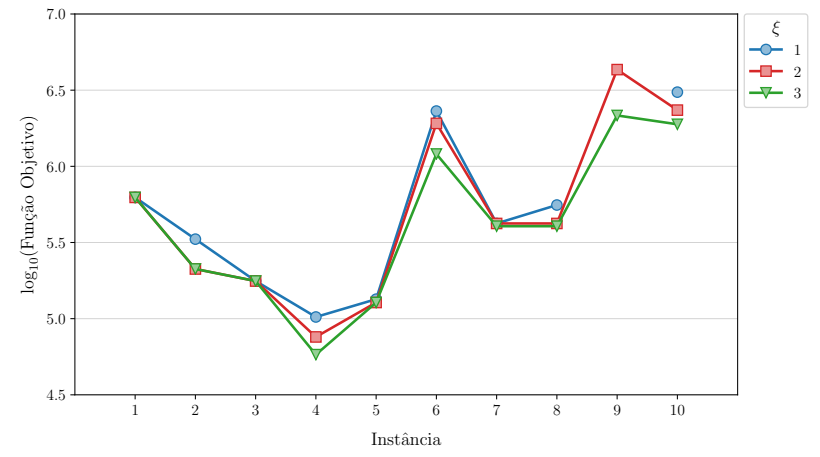

(c) $\mathcal{M}_{1, P}^{3}$

Figura 2.28: Função objetivo encontrada pelos três modelos, considerando as instâncias da Tabela 2.1, para diferentes valores de $\xi$. 
Tabela 2.3: Resultado numérico para as instâncias do PCBMPSA, presentes na Tabela 2.1, para diferentes valores de $\xi$

\begin{tabular}{|c|c|c|c|c|c|c|c|c|c|c|c|c|c|c|c|c|}
\hline \multirow{2}{*}{ Modelo } & \multirow{2}{*}{ Inst. } & \multicolumn{5}{|c|}{$\xi=1$} & \multicolumn{5}{|c|}{$\xi=2$} & \multicolumn{5}{|c|}{$\xi=3$} \\
\hline & & $\begin{array}{l}\text { Função } \\
\text { Objetivo }\end{array}$ & $\begin{array}{l}\text { Custo } \\
\text { Objetos }\end{array}$ & $\begin{array}{l}\text { Valor } \\
\text { Sobras }\end{array}$ & $\operatorname{Gap}(\%)$ & Tempo(s) & $\begin{array}{l}\text { Função } \\
\text { Objetivo }\end{array}$ & $\begin{array}{l}\text { Custo } \\
\text { Objetos }\end{array}$ & $\begin{array}{l}\text { Valor } \\
\text { Sobras }\end{array}$ & $\operatorname{Gap}(\%)$ & Tempo(s) & $\begin{array}{l}\text { Função } \\
\text { Objetivo }\end{array}$ & $\begin{array}{l}\text { Custo } \\
\text { Objetos }\end{array}$ & $\begin{array}{l}\text { Valor } \\
\text { Sobras }\end{array}$ & $\operatorname{Gap}(\%)$ & Tempo(s) \\
\hline \multirow{10}{*}{$\mathcal{M}_{1, P}^{1}$} & 1 & 624.810 & 477 & 60 & - & 0,21 & 624.810 & 477 & 60 & - & 0,91 & 624.810 & 477 & 60 & - & 1,53 \\
\hline & 2 & 332.758 & 412 & 138 & - & 0,07 & 211.619 & 262 & 77 & - & 594,75 & 168.040 & 208 & 24 & - & 855,39 \\
\hline & 3 & 176.480 & 290 & 130 & - & 1,56 & 105.952 & 174 & 14 & - & 6,16 & 100.485 & 165 & 0 & - & 3,73 \\
\hline & 4 & 102.668 & 184 & 4 & - & 0,14 & 75.884 & 136 & 4 & - & 0,50 & 58.032 & 104 & 0 & - & 5,21 \\
\hline & 5 & 134.216 & 204 & 16 & - & 0,22 & 127.592 & 194 & 60 & - & 3,63 & 94.742 & 144 & 10 & - & 48,95 \\
\hline & 6 & 2.305 .307 & 716 & 213 & - & 6,78 & 1.915 .751 & 595 & 149 & - & 0,62 & 1.204 .236 & 374 & 44 & - & $2.037,05$ \\
\hline & 7 & 421.688 & 375 & 187 & - & 0,10 & 421.656 & 375 & 219 & - & 0,89 & 404.802 & 360 & 198 & - & 280,56 \\
\hline & 8 & 556.688 & 495 & 187 & - & 0,13 & 421.677 & 375 & 198 & - & 0,40 & 404.825 & 360 & 175 & - & $1.714,96$ \\
\hline & 9 & Inviável & & & & & 4.319 .619 & 1.200 & 381 & 0,01 & $\dagger$ & 2.159 .727 & 600 & 273 & 0,04 & $\dagger$ \\
\hline & 10 & 3.071 .731 & 1.299 & 404 & - & $2.138,68$ & 2.341 .059 & 990 & 291 & 0,02 & $\dagger$ & 1.889 .489 & 799 & 146 & 0,30 & $\dagger$ \\
\hline \multirow{10}{*}{$\mathcal{M}_{1, P}^{2}$} & 1 & 624.810 & 477 & 60 & - & 0,14 & 624.810 & 477 & 60 & - & 0,55 & 624.810 & 477 & 60 & - & 2,07 \\
\hline & 2 & 332.758 & 412 & 138 & - & 0,12 & 211.619 & 262 & 77 & - & $1.392,43$ & 168.064 & 208 & 0 & - & 4,14 \\
\hline & 3 & 176.480 & 290 & 130 & - & 1,33 & 105.954 & 174 & 12 & - & 0,65 & 105.954 & 174 & 12 & - & 32,42 \\
\hline & 4 & 102.668 & 184 & 4 & - & 0,07 & 75.884 & 136 & 4 & - & 0,54 & 58.032 & 104 & 0 & - & 3,61 \\
\hline & 5 & 134.216 & 204 & 16 & - & 0,23 & 127.592 & 194 & 60 & - & 0,82 & 94.748 & 144 & 4 & - & $1.135,66$ \\
\hline & 6 & 2.305 .307 & 716 & 213 & - & 5,63 & 1.915 .751 & 595 & 149 & - & 0,36 & 1.204 .236 & 374 & 44 & - & $1.914,57$ \\
\hline & 7 & 421.688 & 375 & 187 & - & 0,10 & 421.656 & 375 & 219 & - & 0,22 & 404.802 & 360 & 198 & - & 57,11 \\
\hline & 8 & 556.688 & 495 & 187 & - & 0,15 & 421.677 & 375 & 198 & - & 0,28 & 404.825 & 360 & 175 & - & 687,08 \\
\hline & 9 & Inviável & & & & & 4.319 .643 & 1.200 & 357 & 0,00 & $\dagger$ & 2.159 .727 & 600 & 273 & 0,03 & $\dagger$ \\
\hline & 10 & 3.071 .731 & 1.299 & 404 & - & $1.252,98$ & 2.341 .074 & 990 & 276 & 0,02 & $\dagger$ & 1.889 .468 & 799 & 167 & 0,28 & $\dagger$ \\
\hline \multirow{10}{*}{$\mathcal{M}_{1, P}^{3}$} & 1 & 624.810 & 477 & 60 & - & 0,11 & 624.810 & 477 & 60 & - & 0,45 & 624.810 & 477 & 60 & - & 1,25 \\
\hline & 2 & 332.758 & 412 & 138 & - & 0,06 & 211.619 & 262 & 77 & - & 612,60 & 211.619 & 262 & 77 & - & 110,71 \\
\hline & 3 & 176.480 & 290 & 130 & - & 1,81 & 176.480 & 290 & 130 & - & 3,46 & 176.480 & 290 & 130 & - & 13,16 \\
\hline & 4 & 102.672 & 184 & 0 & - & 0,04 & 75.888 & 136 & 0 & - & 0,17 & 58.032 & 104 & 0 & - & 3,70 \\
\hline & 5 & 134.216 & 204 & 16 & - & 0,18 & 127.607 & 194 & 45 & - & 0,62 & 127.592 & 194 & 60 & 0,02 & $\dagger$ \\
\hline & 6 & 2.305 .307 & 716 & 213 & - & 5,45 & 1.915 .751 & 595 & 149 & - & 0,49 & 1.204 .236 & 374 & 44 & - & 996,32 \\
\hline & 7 & 421.688 & 375 & 187 & - & 0,13 & 421.656 & 375 & 219 & - & 0,23 & 404.810 & 360 & 190 & - & 104,98 \\
\hline & 8 & 556.688 & 495 & 187 & - & 0,11 & 421.677 & 375 & 198 & - & 0,22 & 404.825 & 360 & 175 & - & $1.244,80$ \\
\hline & 9 & Inviável & & & & & 4.319 .661 & 1.200 & 339 & 0,01 & $\dagger$ & 2.159 .727 & 600 & 273 & 0,03 & $\dagger$ \\
\hline & 10 & 3.071 .731 & 1.299 & 404 & - & $2.255,12$ & 2.341 .056 & 990 & 294 & 0,01 & $\dagger$ & 1.889 .498 & 799 & 137 & 0,24 & $\dagger$ \\
\hline
\end{tabular}

$\dagger$ : Tempo limite de $2 \mathrm{~h}$ atingido 
Vamos agora considerar as instâncias presentes na Tabela A.1, que são instâncias maiores (com mais itens, objetos e períodos). Nessas instâncias, as dez primeiras contêm quatro períodos, as próximas dez possuem oito períodos e as dez últimas doze períodos. Como já mencionado, resolver instâncias médias e grandes é uma tarefa árdua para o solver. Por isso, para essas instâncias, consideramos $\xi \in\{1,2,3\}$. Como os três modelos obtiveram, nessas instâncias, as mesmas soluções, apresentamos os resultados apenas do modelo $\mathcal{M}_{1, P}^{2}$. A Tabela 2.4 mostra o resultado obtido em cada instância para os diferentes valores de $\xi$.

Como esperado, para $\xi=1$ praticamente todas as instâncias foram resolvidas gastando menos de 2 horas (tempo limite imposto ao CPLEX). Apenas em quatro instâncias (8, 26, 28 e 30) o tempo limite não foi suficiente para o solver obter a solução ótima. Entretanto, o valor da função objetivo é maior quando comparado $\operatorname{com} \xi>1$, o que é natural (veja o gráfico da Figura 2.29). Na maioria das instâncias, o custo dos objetos utilizados é reduzido quando aumentamos o valor de $\xi$. Por exemplo, na instância 12 o custo dos objetos utilizados foi de 2.132, 1.400 e 1.076, respectivamente, para $\xi=1$, $\xi=2$ e $\xi=3$. Para $\xi=2$ o solver, dentro do tempo limite, não conseguiu a solução ótima para 11 instâncias e para $\xi=3$, esse número é ainda maior, 22. Deixando claro que encontrar a solução ótima dessas 30 instâncias para valores maiores de $3 \leq \xi \leq P-p$ demandaria muito tempo.

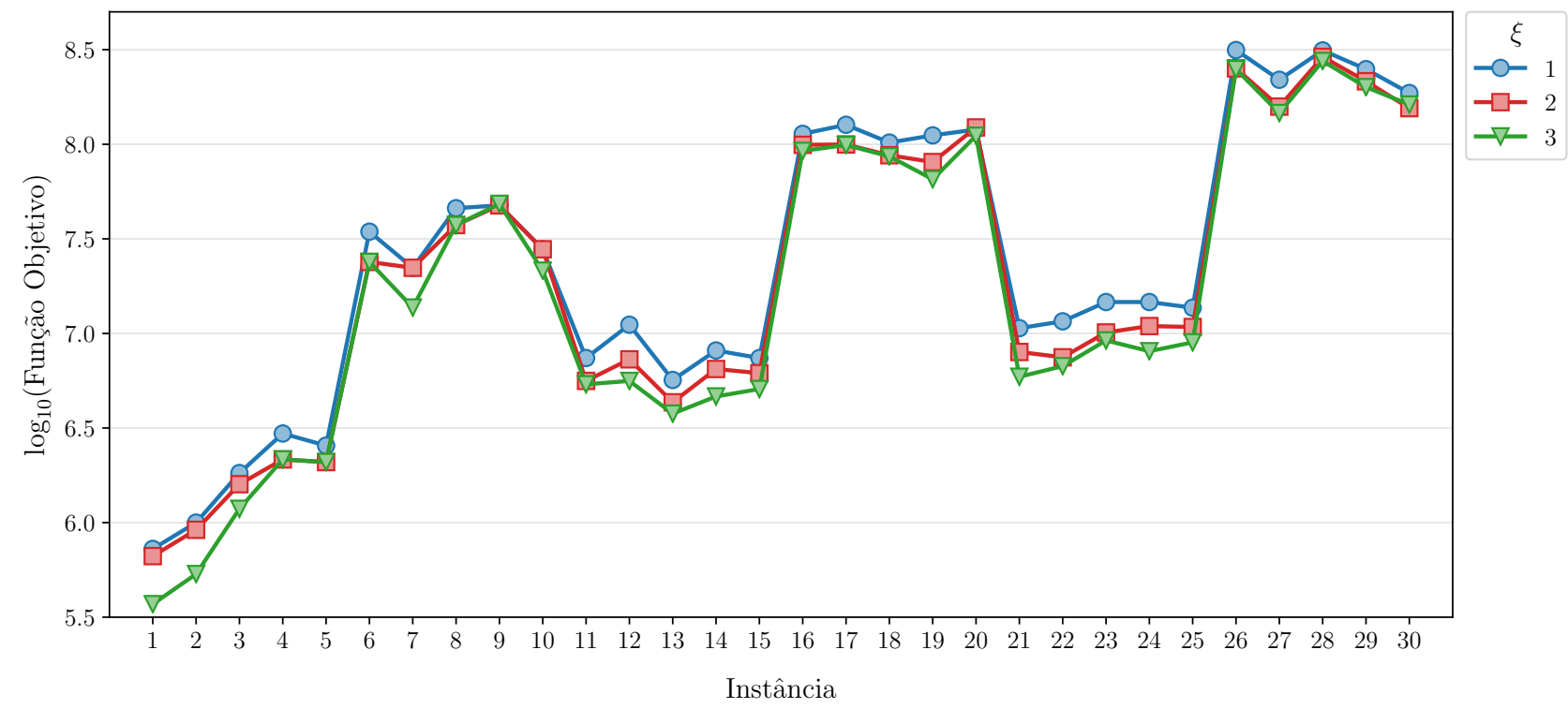

Figura 2.29: Função objetivo encontrada pelo modelo $\mathcal{M}_{1, P}^{2}$, considerando as instâncias da Tabela A.1, para diferentes valores de $\xi$. 
Tabela 2.4: Resultado numérico da solução do $\mathcal{M}_{1, P}^{2}$, para as instâncias presentes na Tabela A.1, considerando diferentes valores de $\xi$.

\begin{tabular}{|c|c|c|c|c|c|c|c|c|c|c|c|c|c|c|c|}
\hline \multirow{2}{*}{ Inst. } & \multicolumn{5}{|c|}{$\xi=1$} & \multicolumn{5}{|c|}{$\xi=2$} & \multicolumn{5}{|c|}{$\xi=3$} \\
\hline & $\begin{array}{l}\text { Função } \\
\text { Objetivo }\end{array}$ & $\begin{array}{l}\text { Custo } \\
\text { Objetos }\end{array}$ & $\begin{array}{l}\text { Valor } \\
\text { Sobras }\end{array}$ & Gap(\%) & Tempo(s) & $\begin{array}{c}\text { Função } \\
\text { Objetivo }\end{array}$ & $\begin{array}{l}\text { Custo } \\
\text { Objetos }\end{array}$ & $\begin{array}{l}\text { Valor } \\
\text { Sobras }\end{array}$ & Gap $(\%)$ & Tempo(s) & $\begin{array}{c}\text { Função } \\
\text { Objetivo }\end{array}$ & $\begin{array}{l}\text { Custo } \\
\text { Objetos }\end{array}$ & $\begin{array}{l}\text { Valor } \\
\text { Sobras }\end{array}$ & Gap(\%) & Tempo(s) \\
\hline 1 & 726.573 & 376 & 235 & - & 0,31 & 664.737 & 344 & 215 & - & 0,16 & 371.057 & 192 & 79 & 0,01 & \\
\hline 2 & 1.000 .484 & 472 & 156 & - & 0,28 & 915.684 & 432 & 156 & - & 0,20 & 534.135 & 252 & 105 & - & $1.595,21$ \\
\hline 3 & 1.827 .536 & 952 & 304 & - & 0,56 & 1.597 .236 & 832 & 204 & - & 1,18 & 1.182 .322 & 616 & 398 & 0,01 & \\
\hline 4 & 2.957 .680 & 740 & 100 & - & 0,34 & 2.158 .190 & 540 & 190 & - & 9,78 & 2.158 .129 & 540 & 251 & - & 221,47 \\
\hline 5 & 2.550 .272 & 801 & 112 & - & 0,23 & 2.091 .677 & 657 & 211 & - & $2.373,88$ & 2.091 .636 & 657 & 252 & 0,01 & \\
\hline 6 & 34.478 .637 & 2.800 & 563 & - & 19,65 & 23.889 .088 & 1.940 & 72 & - & 138,72 & 23.888 .977 & 1.940 & 183 & 0,00 & \\
\hline 7 & 22.209 .939 & 1.995 & 396 & - & 0,80 & 22.209 .831 & 1.995 & 504 & - & 0,91 & 13.715 .629 & 1.232 & 227 & - & $5.260,77$ \\
\hline 8 & 45.936 .200 & 2.792 & 576 & 0,00 & $\dagger$ & 37.413 .879 & 2.274 & 243 & 0,00 & $\dagger$ & 37.413 .879 & 2.274 & 243 & 0,00 & \\
\hline 9 & 47.549 .202 & 2.883 & 117 & - & 101,33 & 47.549 .102 & 2.883 & 217 & 4,16 & $\dagger$ & 48.406 .504 & 2.935 & 451 & 11,68 & \\
\hline 10 & 27.873 .704 & 1.908 & 268 & - & 2,14 & 27.873 .382 & 1.908 & 590 & - & 2,19 & 21.474 .855 & 1.470 & 375 & 0,00 & \\
\hline 11 & 7.409 .270 & 1.458 & 286 & - & 0,46 & 5.605 .213 & 1.103 & 233 & - & 33,59 & 5.386 .722 & 1.060 & 198 & - & 38,93 \\
\hline 12 & 11.116 .028 & 2.132 & 220 & - & 0,34 & 7.299 .358 & 1.400 & 242 & - & 30,13 & 5.610 .264 & 1.076 & 0 & - & 20,82 \\
\hline 13 & 5.672 .967 & 1.381 & 181 & - & 0,49 & 4.329 .788 & 1.054 & 44 & - & 39,73 & 3.762 .884 & 916 & 44 & 0,00 & \\
\hline 14 & 8.126 .100 & 1.606 & 260 & - & 2,28 & 6.491 .660 & 1.283 & 320 & - & 752,97 & 4.644 .920 & 918 & 160 & 0,01 & \\
\hline 15 & 7.412 .653 & 1.425 & 197 & - & 0,18 & 6.169 .375 & 1.186 & 197 & - & 2,27 & 5.082 .073 & 977 & 281 & - & $4.631,09$ \\
\hline 16 & 113.608 .773 & 4.423 & 405 & - & 10,49 & 99.275 .985 & 3.865 & 405 & - & $5.067,48$ & 92.315 .191 & 3.594 & 293 & 7,48 & \\
\hline 17 & 126.745 .130 & 4.979 & 294 & - & $1.013,86$ & 99.660 .036 & 3.915 & 204 & 9,71 & $\dagger$ & 99.099 .566 & 3.893 & 642 & 24,92 & \\
\hline 18 & 102.002 .632 & 4.345 & 588 & - & 6,25 & 87.400 .468 & 3.723 & 680 & 0,00 & $\dagger$ & 86.297 .218 & 3.676 & 558 & 4,57 & \\
\hline 19 & 111.409 .091 & 4.218 & 943 & - & 1,15 & 80.532 .821 & 3.049 & 416 & 0,00 & $\dagger$ & 65.345 .526 & 2.474 & 236 & 0,01 & \\
\hline 20 & 119.613 .046 & 4.298 & 294 & - & $1.555,76$ & 122.869 .236 & 4.415 & 214 & 16,74 & $\dagger$ & 110.958 .021 & 3.987 & 189 & 18,66 & \\
\hline 21 & 10.677 .702 & 1.794 & 186 & - & 1,04 & 7.987.473 & 1.342 & 111 & 0,00 & $\dagger$ & 5.898 .333 & 991 & 99 & 0,00 & \\
\hline 22 & 11.599 .661 & 1.823 & 88 & - & 1,68 & 7.482 .798 & 1.176 & 90 & - & 2,83 & 6.719 .210 & 1.056 & 118 & 0,01 & \\
\hline 23 & 14.661 .631 & 1.844 & 13 & - & 0,21 & 10.105 .629 & 1.271 & 92 & - & 28,74 & 9.191 .343 & 1.156 & 13 & - & 55,16 \\
\hline 24 & 14.653 .851 & 1.933 & 222 & - & 0,70 & 10.946 .888 & 1.444 & 76 & - & 6,81 & 8.058 .527 & 1.063 & 76 & - & 26,04 \\
\hline 25 & 13.670 .428 & 2.244 & 20 & - & 0,30 & 10.825 .350 & 1.777 & 134 & - & 52,58 & 8.967 .224 & 1.472 & 200 & 0,00 & \\
\hline 26 & 314.522 .901 & 7.830 & 369 & 2,76 & $\dagger$ & 251.899 .681 & 6.271 & 118 & 11,85 & $\dagger$ & 249.368 .848 & 6.208 & 304 & 18,74 & \\
\hline 27 & 219.133.432 & 6.104 & 168 & - & 4,22 & 158.175.232 & 4.406 & 168 & - & 840,79 & 146.328 .080 & 4.076 & 320 & 7,86 & \\
\hline 28 & 313.619 .505 & 7.819 & 585 & 0,00 & $\dagger$ & 289.834.372 & 7.226 & 488 & 25,41 & $\dagger$ & 275.555 .191 & 6870 & 509 & 23,77 & \\
\hline 29 & 249.486 .013 & 6.481 & 82 & - & 39,08 & 215.148 .413 & 5.589 & 142 & 18,97 & $\dagger$ & 200.289 .280 & 5203 & 205 & 21,40 & \\
\hline 30 & 186.639.172 & 5.781 & 413 & 0,00 & $\dagger$ & 155.290 .437 & 4.810 & 413 & 3,14 & $\dagger$ & 162.005 .635 & 5018 & 495 & 28,54 & \\
\hline
\end{tabular}

$\dagger$ : Tempo limite de $2 \mathrm{~h}$ atingido 


\section{Capítulo 3}

\section{Métodos de solução para o PCBMPSA}

Sabemos que, na prática, é possível resolver de forma exata apenas instâncias pequenas de modelos MIP, como o apresentado no Capítulo 2, para o problema PCBMPSA. Por conta disso, desenvolver métodos heurísticos para o problema PCBMPSA parece ser o caminho mais natural. No presente capítulo abordamos algumas das técnicas mais comumente utilizadas para problemas de programação linear inteira, como o PCBMPSA. Desta forma, pretendemos dar início ao estudo de métodos que poderiam ser utilizados para resolver instâncias médias ou grandes do problema proposto.

\subsection{Método guloso}

Os métodos gulosos, também chamados de míopes, são algoritmos que fazem uma escolha localmente ótima em cada etapa, sem se preocupar com o futuro, na esperança de que essa escolha leve a encontrar uma solução de boa qualidade. Nesses métodos, quando uma escolha é feita, ela nunca é revista. Em muitos problemas, é natural que essa estratégia não encontre a solução ótima para o problema geral e, em alguns casos, pode não encontrar uma solução viável para o problema. Entretanto, normalmente, são métodos simples, rápidos e de fácil implementação.

Uma estratégia gulosa para solucionar o PCBMPSA é resolver o problema considerando cada período de forma individual, ou seja, resolvendo o modelo $\mathcal{M}_{s, s}^{2}$, para cada $s=p, \ldots, P$, e as possíveis sobras aproveitáveis ficam disponíveis no período seguinte como novos objetos de custo zero, tendo assim, uma solução ótima para cada período. Observe que essa abordagem corresponde a resolver, para cada período, o modelo proposto para o PCSA, apresentado na Seção 2.1. O Algoritmo 3.1 ilustra o pseudocódigo de um método guloso proposto para o esse problema. O algoritmo recebe como entrada doze parâmetros, a saber:

- $p$ e $P$ : períodos inicial e final em que o método guloso será aplicado;

- $\tilde{n}_{s}$ : número de itens distintos no período $s=p, \ldots, P$;

- $n_{s}$ : número total de itens da ordem de trabalho no período $s=p, \ldots, P$; 
- $w_{s i}$ e $h_{s i}$ : largura e altura do item $i=1, \ldots, n_{s}$ no período $s=p, \ldots, P$;

- $d$ : número de itens do catálogo;

- $\bar{w}_{i}$ e $\bar{h}_{i}$ : largura e altura do item $i=1, \ldots, d$ do catálogo;

- $m_{s}$ : número de objetos disponíveis no período $s=p, \ldots, P$;

- $W_{s j}$ e $H_{s j}$ : largura e altura do objeto $j=1, \ldots, m_{s}$ no período $s=p, \ldots, P$.

O Algoritmo 3.1 começa definindo $\bar{m}_{s}$, que representa o número total de objetos do período $s$, ou seja, os $m_{s}$ objetos de entrada mais o número máximo de sobras geradas no período anterior. Em seguida, para cada período $s$, o algoritmo resolve o modelo $\mathcal{M}_{s, s}^{2}$. Para cada objeto $j=1, \ldots, \bar{m}_{s}$, definimos a largura da sobra da direita $\left(r_{s j}\right)$ e a altura da sobra superior $\left(t_{s j}\right)$ e, se $s<P$, calculamos as dimensões e o valor por unidade de área de cada sobra aproveitável, usando o Algoritmo 3.2. Tais sobras ficam disponíveis no próximo período como objetos com custo zero. Ao fim, o algoritmo retorna, para cada objeto $j$, as seguintes informações: se o mesmo foi utilizado ou não, o tipo de corte guilhotinado (Caso A ou Caso B) do objeto, a largura da sobra da direita e a altura da sobra superior do objeto, em qual objeto cada item foi atribuído, a posição $x$ e $y$ de cada item dentro do objeto e as dimensões e custo por unidade de área de todos os objetos, ou seja, dos objetos de entrada e dos objetos originados de sobras.

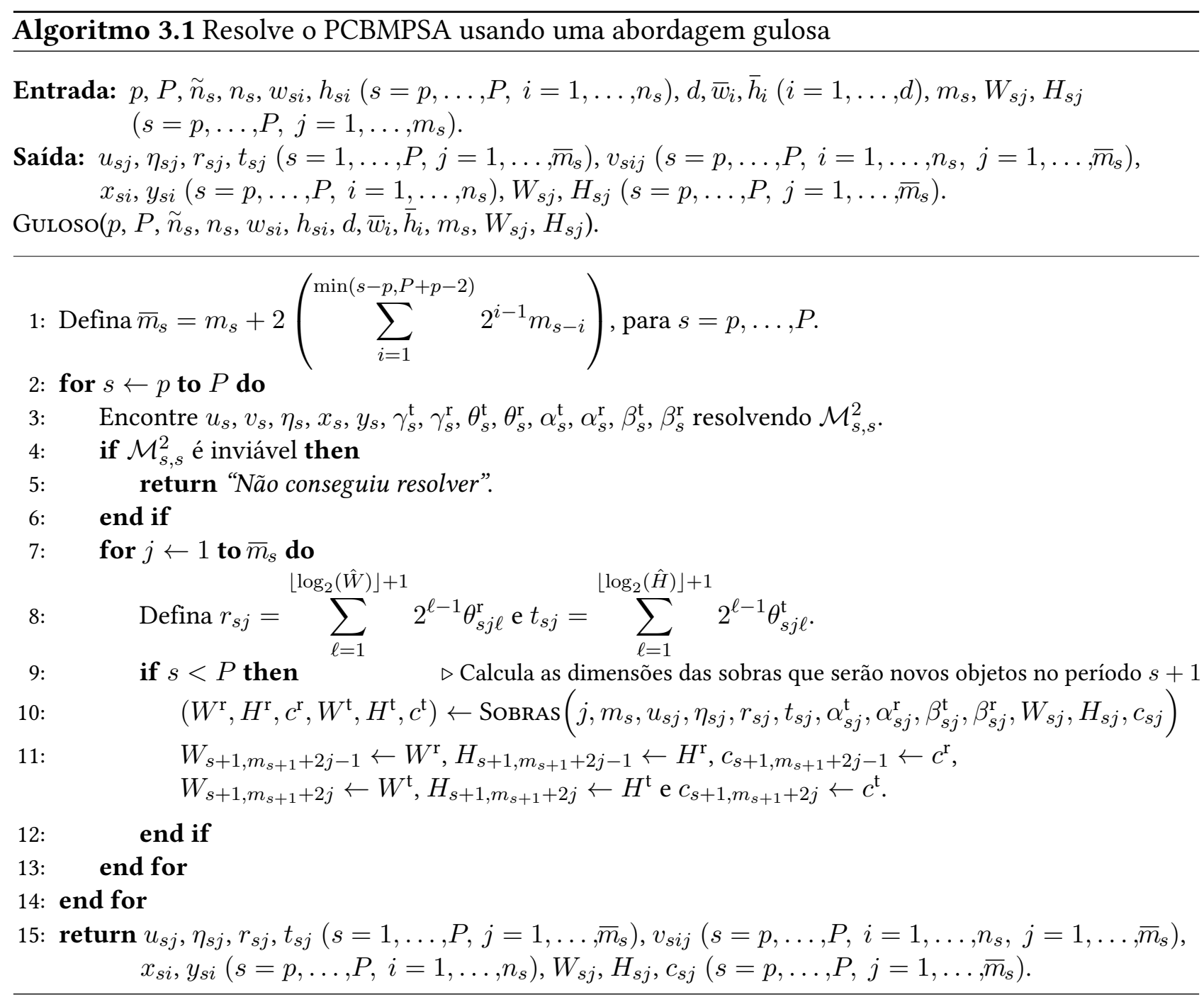


O Algoritmo 3.2 apresenta o pseudocódigo para calcular as dimensões das sobras de cada objeto. $\mathrm{O}$ algoritmo recebe como entrada os seguintes parâmetros:

- $j$ : índice (posição) do objeto;

- $m$ : número de objetos de entrada;

- $u$ : variável binária que vale 1 se o objeto foi utilizado e 0 caso contrário;

- $\eta$ : variável binária que vale 0 se o objeto foi cortado seguindo o Caso A e 1 para o Caso B;

- $r$ : variável associada à largura da sobra da direita do objeto;

- $t$ : variável associada à altura da sobra superior do objeto;

- $\alpha^{\mathrm{t}}$ e $\alpha^{\mathrm{r}}$ : área da sobra superior e da sobra da direita, respectivamente, se o Caso A for utilizado no objeto;

- $\beta^{\mathrm{t}}$ e $\beta^{\mathrm{r}}$ : área da sobra superior e da sobra da direita, respectivamente, se o Caso B for utilizado no objeto;

- $W, H, c$ : largura, altura e custo por unidade de área, respectivamente, do objeto.

Observe que $u, \eta, r, t, \alpha^{\mathrm{t}}, \alpha^{\mathrm{r}}, \beta^{\mathrm{t}} \mathrm{e} \beta^{\mathrm{r}}$ são variáveis obtidas ao se resolver o modelo $\mathcal{M}_{s, s}^{2}$, no período $s=p, \ldots, P$. Entre as linhas 1 e 21 do Algoritmo 3.2, calculamos as dimensões da sobra da direita do objeto. Se o objeto foi utilizado $(u=1)$, de acordo com o tipo de corte guilhotinado (Caso A ou Caso B), verificamos se o objeto possui sobra da direita aproveitável, ou seja, se a área da sobra é maior que zero. Caso tenha sido usado, definimos as dimensões da sobra de acordo com o tipo de corte e atribuímos ao valor por unidade de área da sobra o custo por unidade de área do objeto ao qual ela foi originada. Caso o objeto não tenha sido utilizado $(u=0)$ verificamos se ele é um objeto originado de uma sobra anterior, ou seja, se seu índice $j$ é maior que $m$. Se for, essa sobra deve ficar disponível no próximo período (lembrando que quando um objeto originado de uma sobra anterior não é utilizado, ele é posicionado no próximo período na mesma posição de sua sobra da direita). Caso contrário, o objeto não foi utilizado e não é originado de uma sobra anterior e, portanto, definimos a largura, a altura e o valor por unidade de área de sua sobra como zero. De forma muito similar ao que foi feito para a sobra da direita, entre as linhas 22 e 38, definimos as dimensões e o valor por unidade de área da sobra superior do objeto. Ao fim, são retornados: a largura, a altura e o valor por unidade de área (correspondente ao custo por unidade de área do objeto ao qual a sobra foi originada) da sobra da direita e da sobra superior.

Como ilustrado no Capítulo 2, uma estratégia gulosa nem sempre encontra uma solução ótima global, podendo, em alguns casos, sequer encontrar uma solução viável para o problema. Isso porque decisões tomadas no presente podem ter consequências no futuro e, devido a tais consequências futuras, uma abordagem gulosa pode ter um desempenho ruim ao fim do horizonte de planejamento [13]. Portanto, precisamos de métodos que consigam antecipar ou prever informações futuras para encontrarmos soluções de melhor qualidade. Dessa forma, a seguir apresentamos outras abordagens que tentam encontrar soluções (melhores que uma solução gulosa) usando informações futuras. 


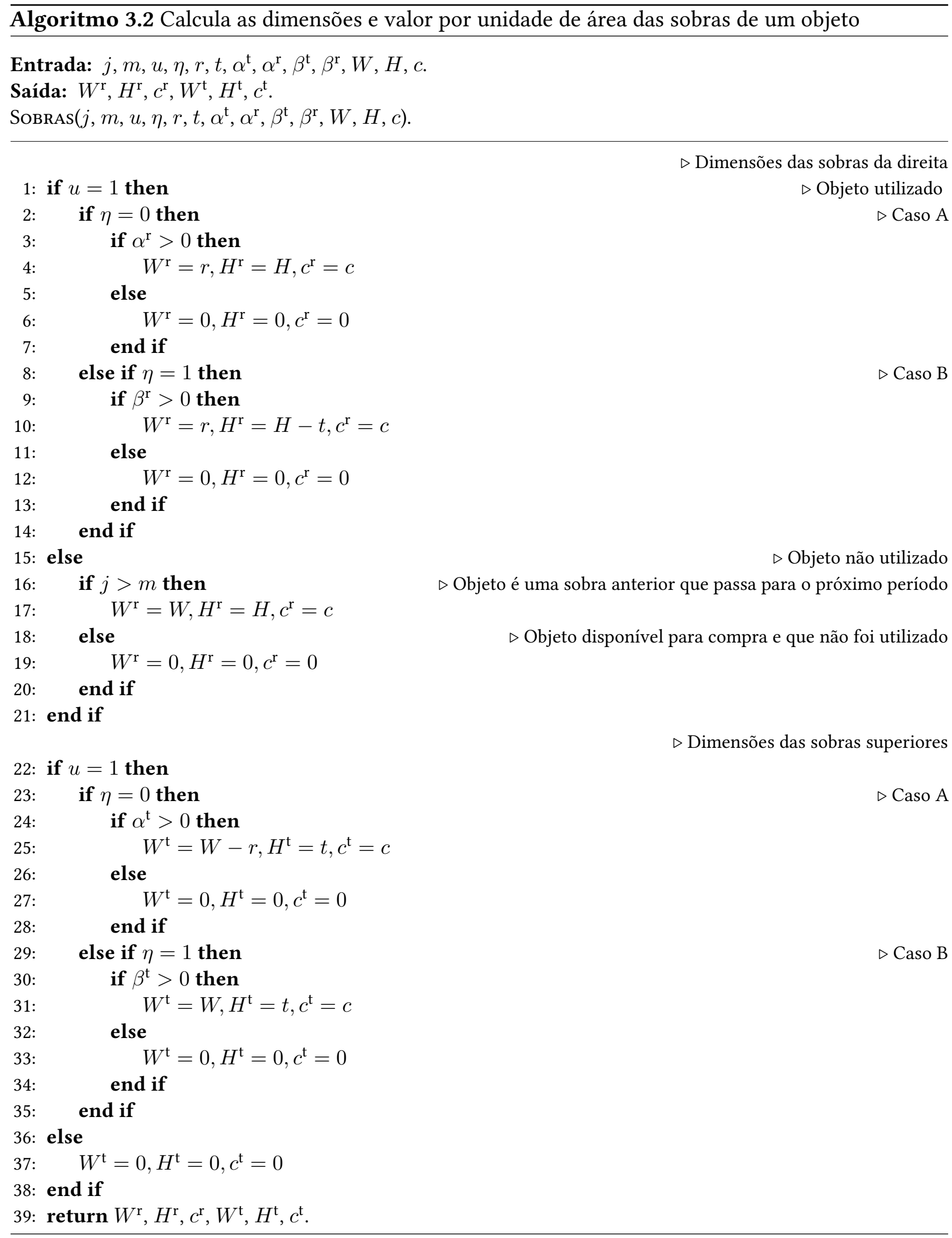




\subsection{Horizonte rolante}

A principal razão para não conseguirmos resolver problemas multiperíodo de forma ótima é devido ao tamanho dos problemas, principalmente quando consideramos dados reais. Uma estratégia de aproximação natural é tentar resolver o problema multiperíodo sobre um horizonte de planejamento menor que o proposto originalmente.

Suponha que estejamos no período $s, p \leq s \leq P$, e que o problema possa ser resolvido de forma eficiente para o horizonte de planejamento de $i=s$ até $f=s+(\Delta-1)$, onde $\Delta \geq 1$ é um número suficientemente pequeno de períodos. Assim, podemos resolver o problema proposto sobre o horizonte de planejamento de $i$ até $f$. Em seguida, fixamos as variáveis do passo anterior e repetimos o processo, mas agora resolvendo o problema para o horizonte $i=f+1$ até $f=i+\Delta-1$, e assim por diante, até que tenhamos resolvido o problema para todos os períodos. Como podemos perceber, em cada passo "rolamos" o horizonte $\Delta$ períodos para frente. Esse método é conhecido como horizonte rolante $(H R)$ [39].

No horizonte rolante, o horizonte de planejamento é dividido em três partes, como mostra a Figura 3.1. Na primeira (chamada de janela congelada) as variáveis de decisão, pertencentes aos períodos dessa parte, tem seus valores fixados. A segunda parte (janela de decisão) contém os dados dos períodos que serão resolvidos. A última parte (janela ignorada) contém os dados que ainda não foram considerados.

A Figura 3.2 exemplifica este processo considerando $p=1, P=12$ e $\Delta=5$. Como podemos notar, no primeiro passo não temos a janela congelada e no último passo a janela de decisão não necessariamente tem $\Delta$ períodos. Além disso, aumentar o valor de $\Delta$ significa aumentar a dificuldade de cada subproblema, já que seu tamanho é aumentado. Perceba que, se $\Delta=1$, então temos o Método Guloso (Seção 3.1). Por outro lado, se $\Delta=P-p+1$, então voltamos ao problema multiperíodo original.

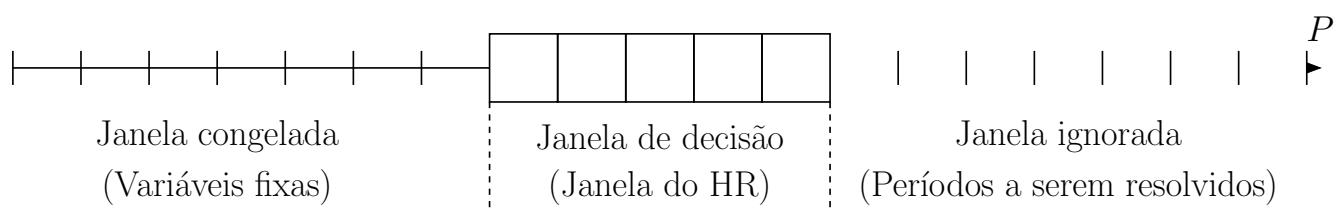

Figura 3.1: Decomposição baseada no horizonte rolante de um problema multiperíodo (baseada em $[1])$.

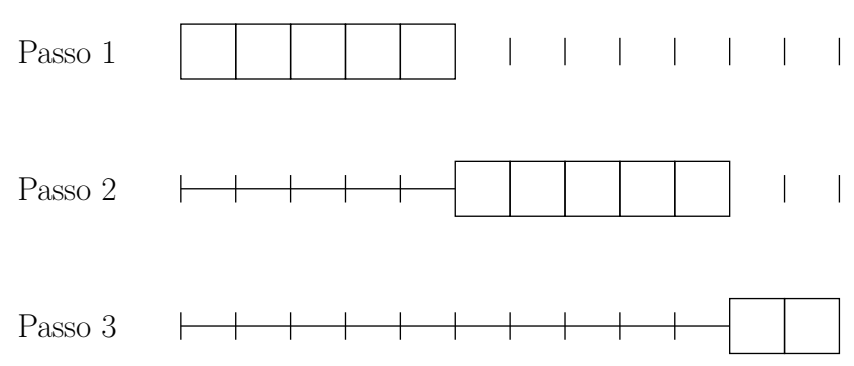

Figura 3.2: Exemplo da abordagem baseada em horizonte rolante para uma instância com 12 períodos e considerando a janela do $H R(\Delta)$ igual a 5 (baseada em [1]). 
Para exemplificar a aplicação do horizonte rolante no problema abordado, considere uma instância como ilustrada pela Figura 3.3 e suponha que o custo por unidade de área de qualquer objeto seja 1. Aplicando a técnica de horizonte rolante com $\Delta=2$, o primeiro passo consiste em resolver o problema considerando os dois primeiros períodos, como mostra a Figura 3.4a. Em seguida, fixamos a solução encontrada e avançamos a janela de decisão do $H R$. Como só temos um período restante, a segunda iteração resolve o problema considerando apenas o terceiro período (Figura 3.4b). A solução final encontrada pelo horizonte rolante possui custo igual a 61.

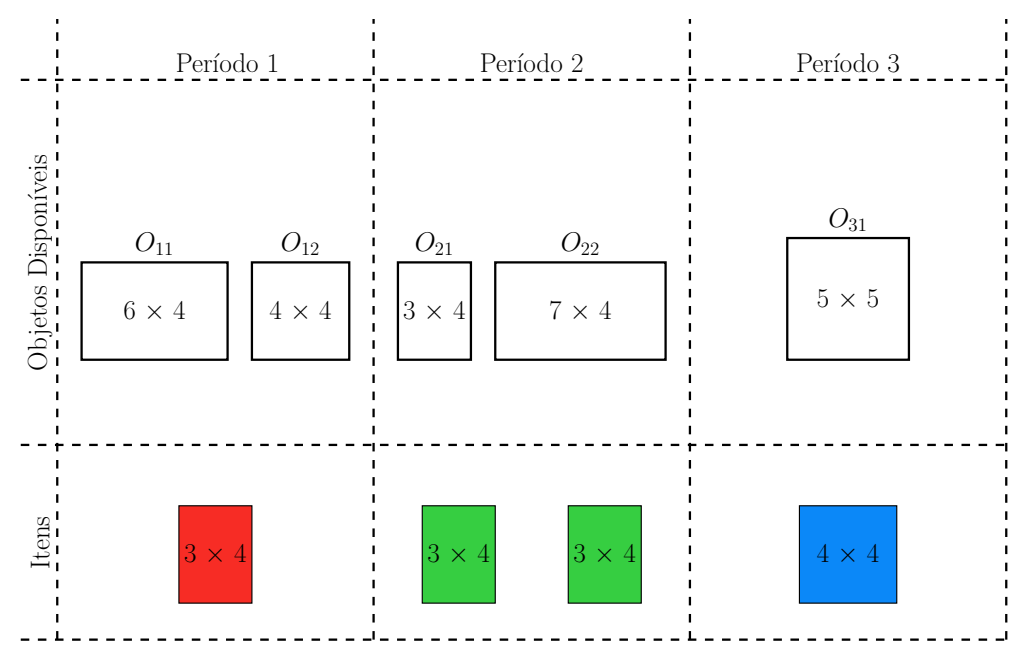

Figura 3.3: Instância ilustrativa a ser usada pelo horizonte rolante.

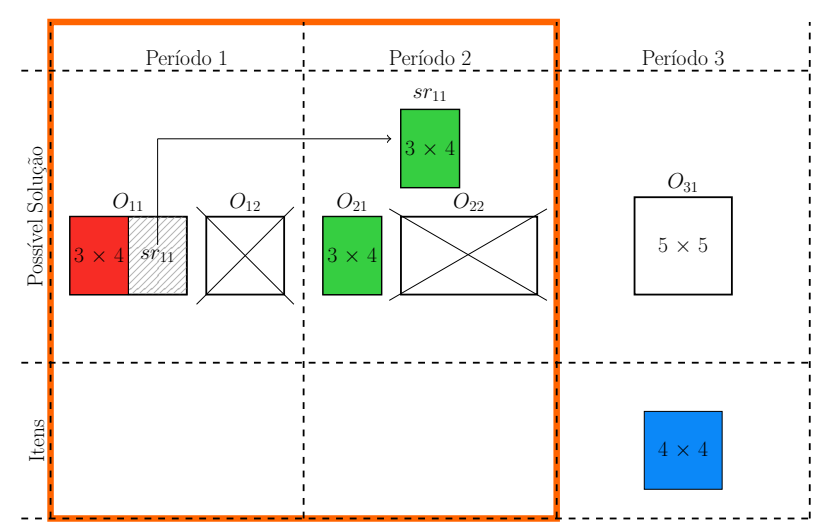

(a) Passo 1 do HR.

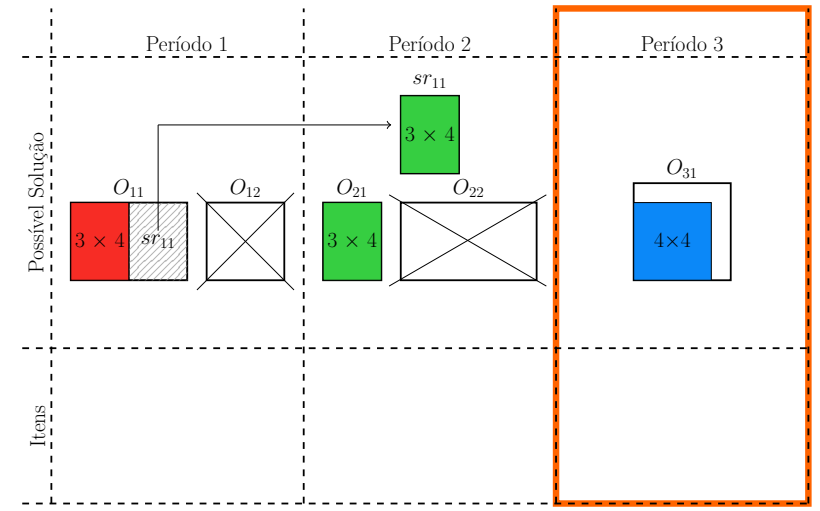

(b) Passo 2 do HR.

Figura 3.4: Horizonte rolante, considerando $\Delta=2$, aplicado a instância da Figura 3.3, cuja solução possui custo igual a 61. A figura da esquerda ilustra o primeiro passo do $H R$, onde apenas os dois primeiros períodos são considerados. Em seguida, fixa-se a solução do passo anterior e o processo é repetido para o terceiro período, como mostrado pela figura da direita.

Além da forma tradicional apresentada para o horizonte rolante, também é comum permitir sobreposição do horizonte de planejamento a ser resolvido entre duas janelas de decisão consecutivas. Assim, na iteração $k+1$, ao invés de fixarmos as variáveis dos $\Delta$ períodos (resolvidos pela iteração $k$ ), fixamos $\Delta-\Phi$, onde $0 \leq \Phi<\Delta$ é o número de períodos sobrepostos. A Figura 3.5 ilustra o horizonte rolante com sobreposição, considerando $\Delta=5$ e $\Phi=2$. Como temos sobreposição de períodos entre duas janelas de decisão consecutivas do $H R$, o tamanho da janela congelada diminui 
e, consequentemente, o número de iterações necessárias para resolver o problema aumenta quando comparado à abordagem em que não temos sobreposição (ou seja, quando $\Phi=0$ ). A Figura 3.6 mostra o horizonte rolante com sobreposição de períodos para $p=1, P=12, \Delta=5$ e $\Phi=2$. Perceba que agora foi preciso uma iteração a mais em relação a Figura 3.2. Entretanto, permitindo sobreposição de períodos, podemos conseguir uma solução global de melhor qualidade, já que diminuímos as variáveis que devem ser fixadas em cada iteração.

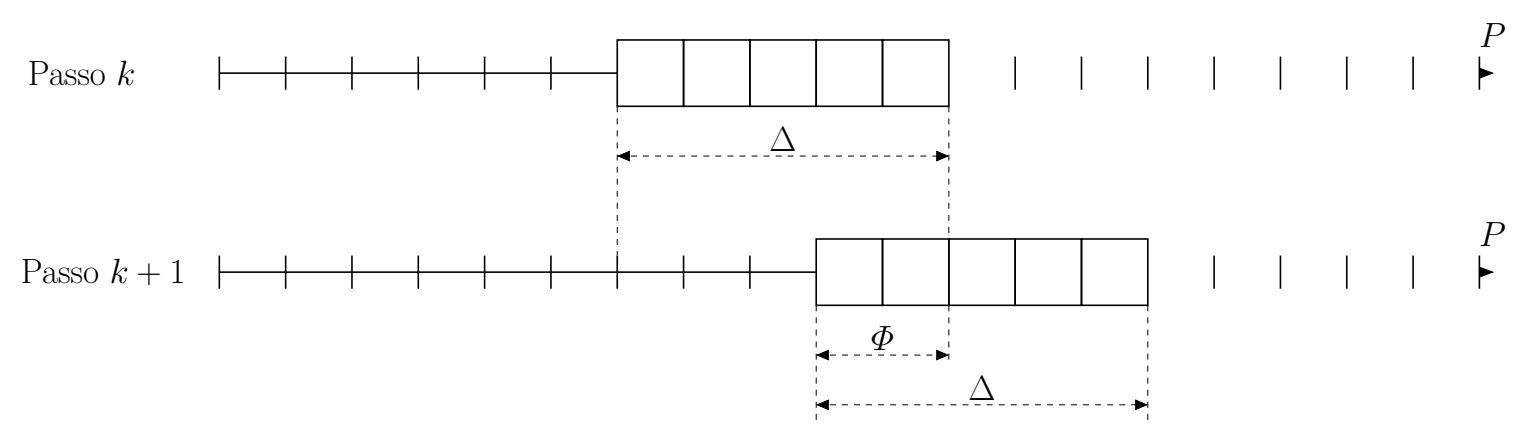

Figura 3.5: Horizonte rolante com sobreposição de períodos a ser resolvido entre duas janelas de decisão consecutivas (baseada em [1]).

Aplicaremos novamente o horizonte rolante na instância da Figura 3.3, mas agora permitindo sobreposição. Considere $\Delta=2$ e $\Phi=1$. Como feito anteriormente, o primeiro passo consiste em resolver os dois primeiros períodos. Em seguida, avançamos a janela do HR. Como estamos considerando sobreposição, agora avançamos $\Delta-\Phi=1$ período e, assim, fixamos apenas as variáveis do primeiro período e descartamos os valores das variáveis do segundo período, pois ele será resolvido novamente na próxima iteração. No segundo passo, resolvemos nosso problema, mas agora considerando os dados do segundo e terceiro período. Observe que a sobra gerada pelo objeto do primeiro período é considerada na resolução do segundo passo. Ao final, permitindo a sobreposição, encontramos uma solução com custo igual a 52, que é melhor que a solução encontrada quando não consideramos sobreposição, mostrada na Figura 3.4. Na verdade, esta solução coincide com a solução ótima da instância.

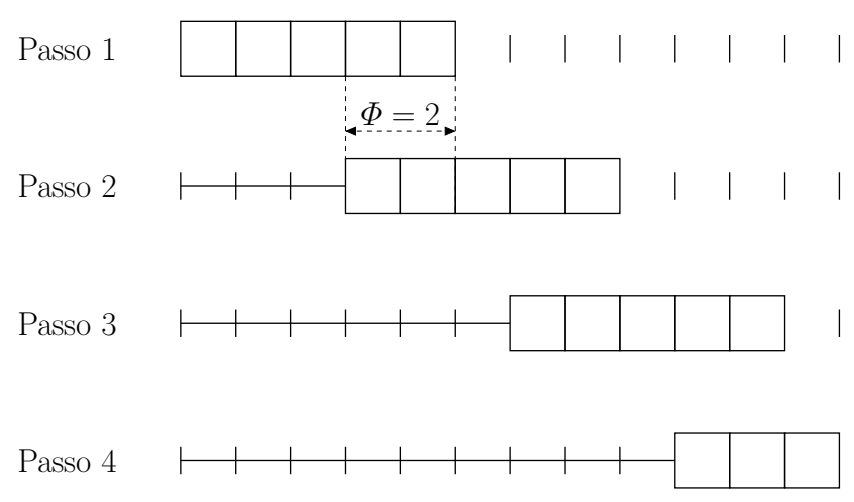

Figura 3.6: Exemplo do horizonte rolante com sobreposição de períodos, considerando a janela do $H R(\Delta)$ igual a 5 e a sobreposição de períodos $(\Phi)$ igual a 2 (baseada em [1]). 


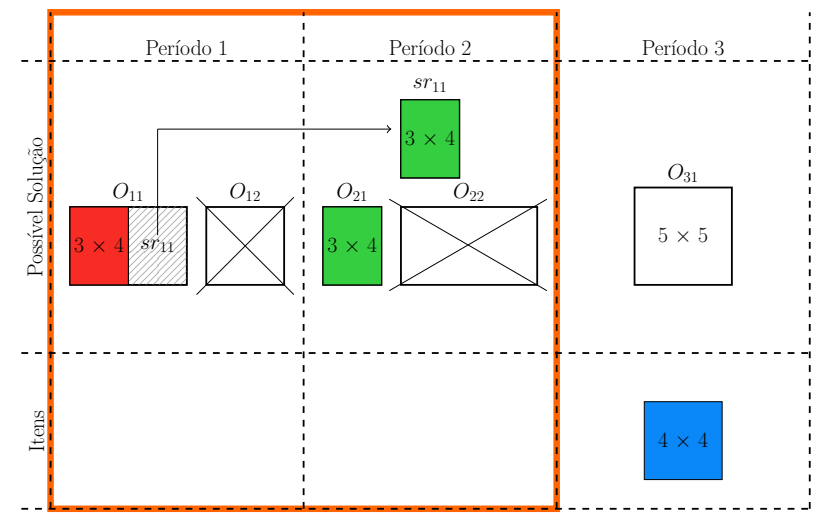

(a) Passo 1 do HR

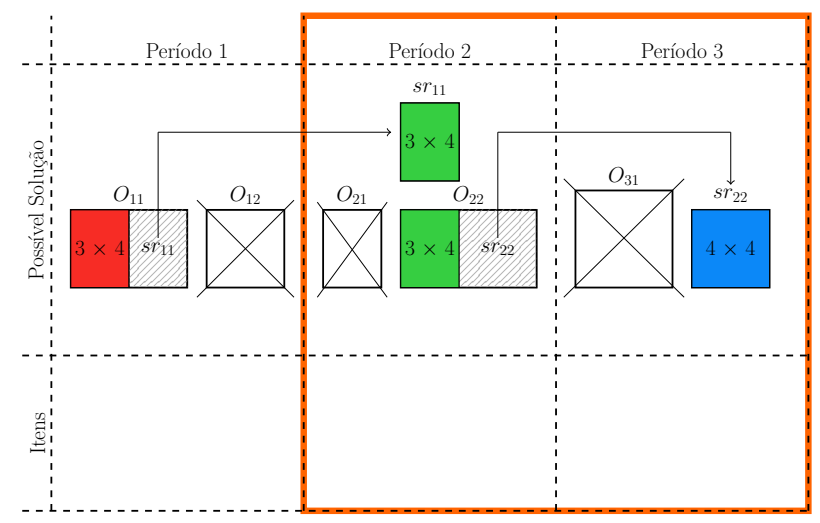

(b) Passo 2 do HR

Figura 3.7: Horizonte rolante com sobreposição, considerando $\Delta=2$ e $\Phi=1$, aplicado a instância da Figura 3.3, cuja solução possui custo igual a 52.

Naturalmente, a solução obtida usando o horizonte rolante pode não ser ótima. Além disso, aumentar o tamanho da janela do horizonte rolante e/ou permitir sobreposição não é garantia de obter solução de melhor qualidade [9]. O Algoritmo 3.3 apresenta um pseudocódigo do horizonte rolante para o nosso problema.

O Algoritmo 3.3 recebe quatorze parâmetros de entrada, sendo os doze primeiros iguais ao do Algoritmo 3.1, seguido pelo tamanho da janela do horizonte rolante $(\Delta)$ e o número de períodos sobrepostos $(\Phi)$. Como podemos observar, o algoritmo é bem simples: primeiramente ele define o valor de $\bar{m}_{s}$, o índice do primeiro e do último período da janela de decisão e, em seguida, resolve o modelo $\mathcal{M}_{i, f}^{2}$ até que $f>P$, sendo que em cada passo os valores de $i$ e $f$ são atualizados de acordo com $\Delta$ e $\Phi$. Lembrando que, no modelo $\mathcal{M}_{i, f}^{2}$, toda aparição de $r_{f j}$ e $t_{f j}$ deve ser substituída por suas representações binárias (2.39) e (2.40), respectivamente, e os produtos $r_{f j} t_{f j}, \bar{W}_{f j} t_{f j}$ e $r_{f j} \bar{H}_{f j}$ devem ser substituídos por suas respectivas representações MIP, dadas por (2.42), (2.43) e (2.44).

Note que o número de iterações, que é a quantidade de subproblemas que devem ser resolvidos, é dado por:

$$
\# \text { Iter }=\left\lceil\frac{(P-p+1)-\Phi}{\Delta-\Phi}\right\rceil
$$




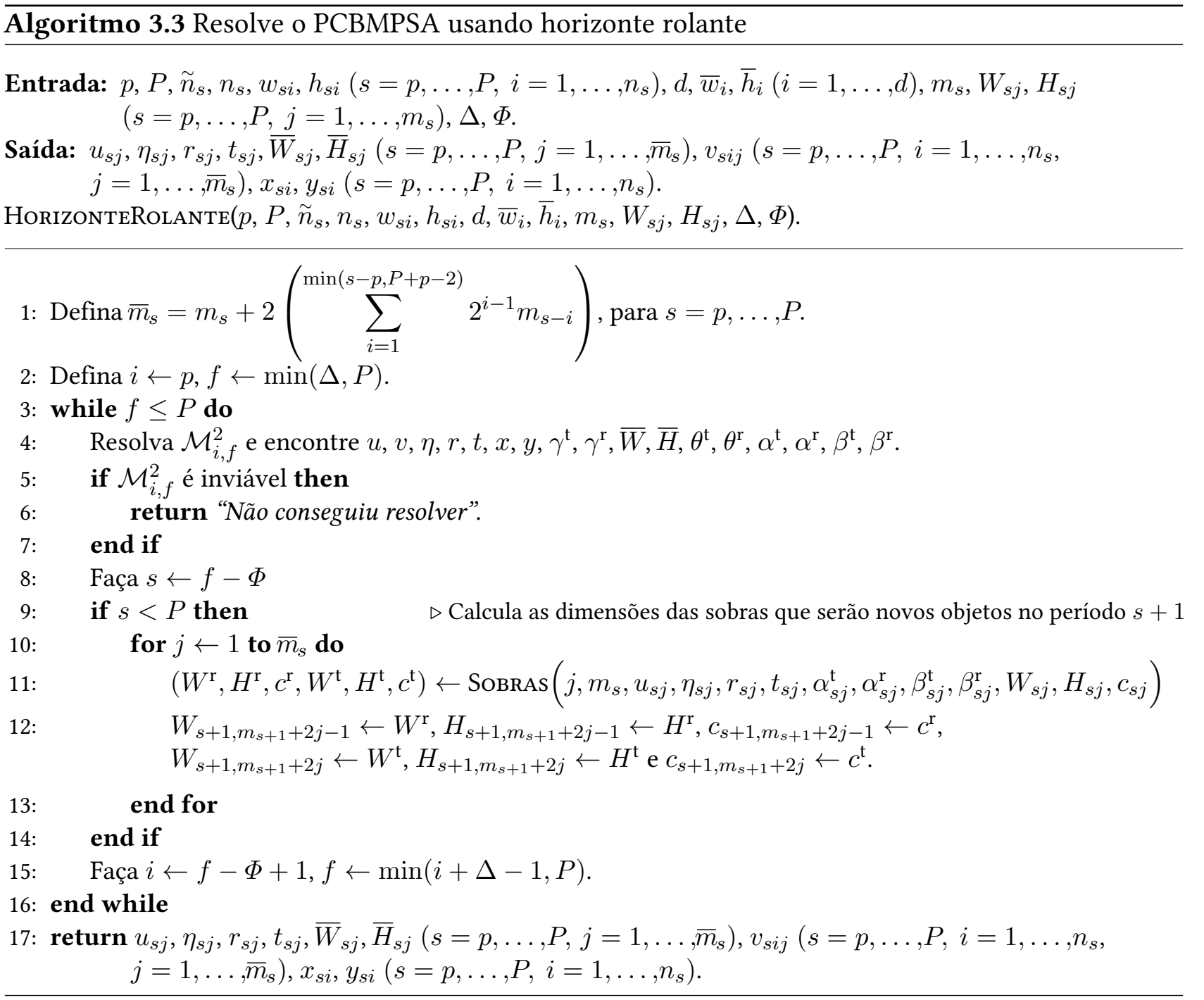

\subsection{Relax-and-fix}

Outra abordagem comumente utilizada na literatura para resolver de forma heurística problemas de otimização computacionalmente difíceis é a heurística relax-and-fix [54]. A abordagem relax-andfix (R\&F) decompõe um problema MIP em vários subproblemas MIP menores do que o problema original e parcialmente relaxados, que são resolvidos em sequência. Em cada subproblema MIP, apenas variáveis de um subconjunto permanecem com a restrição de integralidade, enquanto que as variáveis restantes ou são fixadas ou sua restrição de integralidade é relaxada. Já que o número de variáveis inteiras é reduzido, em relação ao problema original, resolver cada subproblema MIP tende a ser computacionalmente menos custoso.

A heurística relax-and-fix, adotada para resolver o PCBMPSA, se assemelha muito ao horizonte rolante, apresentado na seção anterior. A diferença é que a janela ignorada do horizonte rolante passa a ser uma janela de aproximação e as variáveis dos períodos dessa janela têm sua integralidade relaxada, como ilustra a Figura 3.8. Dessa forma, com a janela de aproximação, podemos conseguir uma aproximação das informações futuras. A Figura 3.9 ilustra o status das variáveis em cada iteração para uma instância com 12 períodos, considerando $\Delta=5$ e $\Phi=2$. Note que, assim como no horizonte 
rolante, também é possível a sobreposição de períodos na janela de decisão do relax-and-fix.

Vale destacar que o subproblema resolvido pela primeira iteração (digamos $R F^{1}$ ) tem duas características principais em comparação aos outros subproblemas: (i) uma vez que $R F^{1}$ é uma relaxação do problema original, a solução ótima obtida por $R F^{1}$ é um limitante inferior válido para a solução ótima do problema original. Entretanto, isto não é válido para os outros subproblemas, já que, heuristicamente, fixamos os valores de algumas variáveis inteiras. (ii) Caso $R F^{1}$ seja inviável, então o problema original também é inviável. Entretanto, se algum outro subproblema for inviável, não podemos concluir que o problema original é inviável [39].

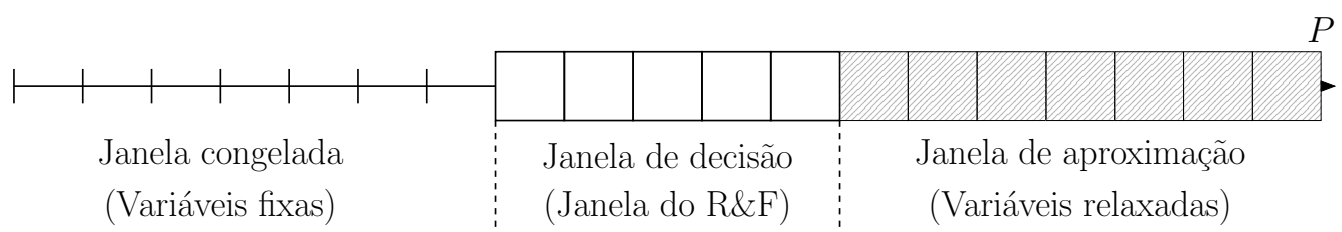

Figura 3.8: Decomposição baseada na abordagem relax-and-fix (baseada em [1]).

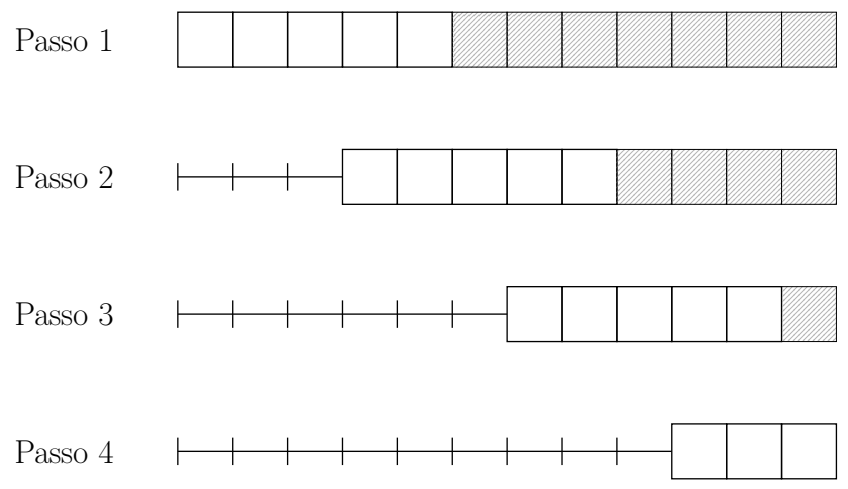

Figura 3.9: Iterações da heurística relax-and-fix para uma instância com 12 períodos e considerando $\Delta=5$ e $\Phi=2$ (baseada em [1]).

Alguns problemas podem ser computacionalmente difíceis de se resolver em tempo aceitável, mesmo relaxando várias variáveis. Nesse caso, podemos usar a heurística relax-and-fix, mas, ao invés de se relaxar a integralidade de todas as variáveis que devem ser inteiras, relaxamos apenas um conjunto pequeno e todas as outras, que deveriam ser relaxadas, são completamente ignoradas [39].

\subsection{Programação dinâmica aproximada}

A programação dinâmica pode ser definida como um método para resolver problemas complexos de tomada de decisão, dividindo-os em um conjunto de subproblemas menores e mais simples de se revolver do que o problema original. Após resolver as diferentes partes do problema (subproblemas), as soluções dos subproblemas são combinadas para encontrar a solução global do problema original. Uma característica comum, ao se dividir o problema original em subproblemas, é que muitos desses são gerados e resolvidos várias vezes. A programação dinâmica procura resolver cada subproblema uma única vez: ao resolver um subproblema, sua solução é armazenada em uma tabela associada ao 
subproblema e na próxima vez que o mesmo tiver de ser resolvido, basta pegar a sua solução já calculada. Dessa forma, se reduz o tempo computacional de se resolver o problema global, principalmente quando o número de subproblemas repetidos cresce exponencialmente [9].

Usando programação dinâmica, o problema que abordamos pode ser modelado da seguinte maneira: geram-se todas as soluções viáveis para o primeiro período. Em seguida, para cada solução viável do primeiro período, fixam-se as variáveis, as sobras aproveitáveis são consideradas objetos do segundo período e, na sequencia, geram-se todas as possíveis soluções viáveis para o segundo período. Repete-se o processo anterior para todos os $P-p+1$ períodos. Em cada período, resolve-se um subproblema bem menor que o problema geral, mas o número de subproblemas a ser resolvido pode crescer rapidamente. A Figura 3.10a ilustra um exemplo hipotético de um grafo de transição para uma instância do nosso problema. Os valores abaixo de cada aresta representam os custos das soluções, onde inf significa que os objetos disponíveis no período não comportam os itens da ordem de trabalho e o modelo torna-se inviável. No primeiro período, duas soluções viáveis foram geradas, solução A com custo 60 e solução B com custo 64 . Em seguida, para cada uma dessas duas soluções, são geradas as soluções viáveis para o segundo período. A partir da solução A, pode-se gerar três soluções (C, D e E) e da solução B pode-se gerar duas soluções (F e G). No último período, a partir de cada uma das cinco soluções encontradas pelo segundo período, nove soluções viáveis são geradas e um subproblema (J) foi inviável.

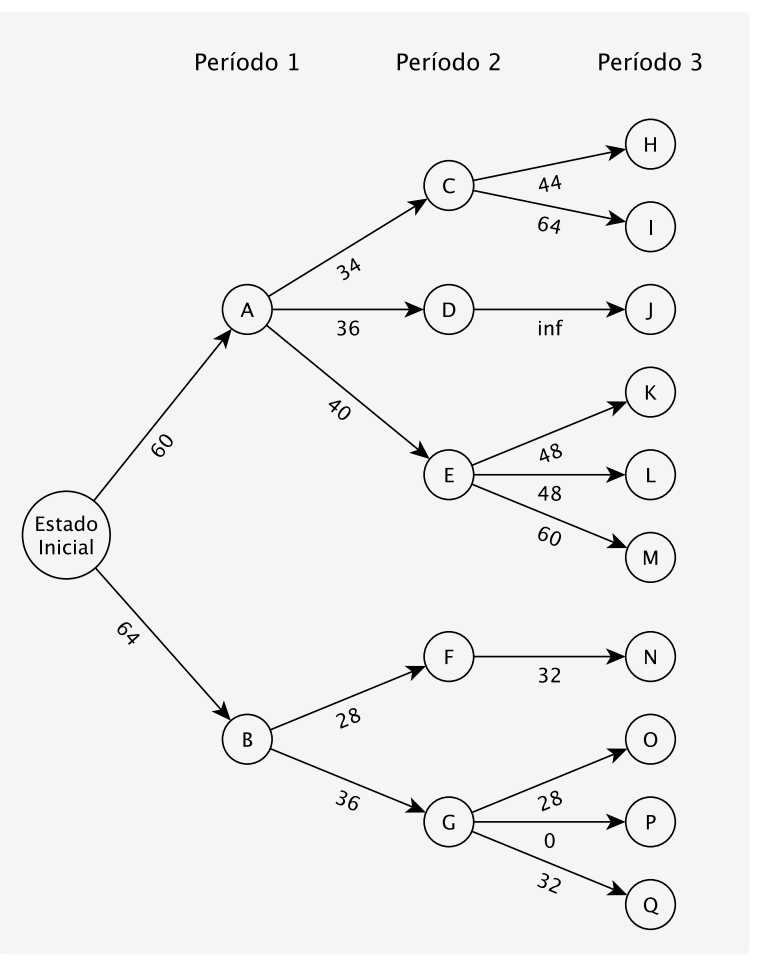

(a)

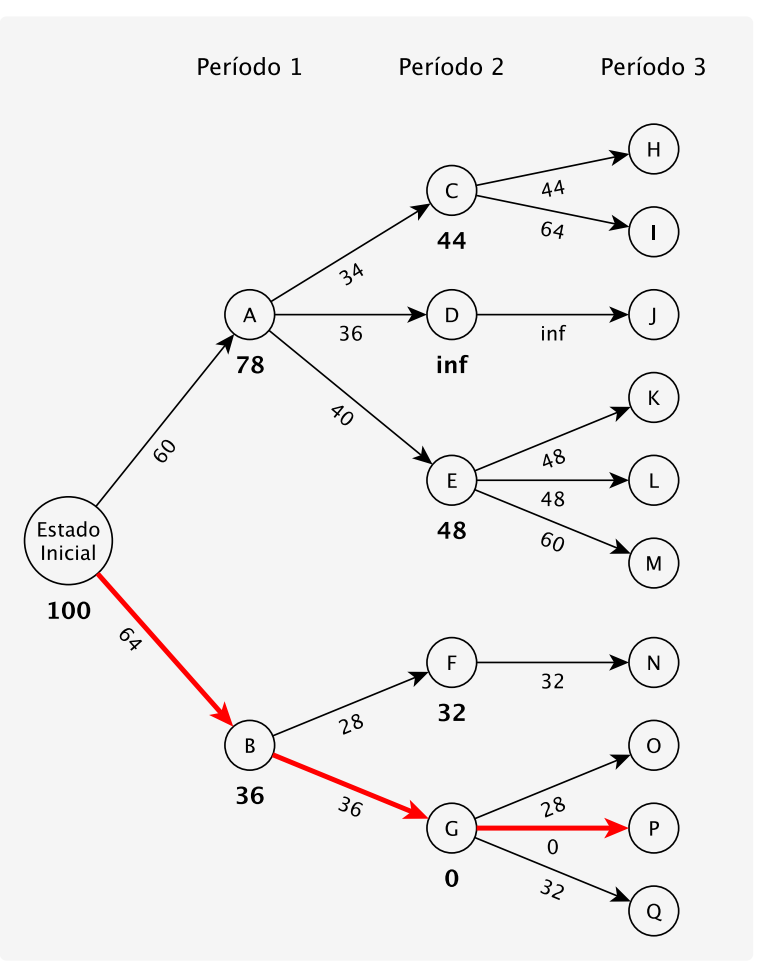

(b)

Figura 3.10: Grafo de transição

Após gerar todas as possíveis soluções de cada período, devemos escolher o caminho cuja soma dos custos seja a menor possível, ou seja, devemos calcular o menor caminho do estado inicial até o 
último período. Para isso, calculamos o menor custo das arestas do terceiro período para cada solução do segundo período, como mostra a Figura 3.10b, esse menor custo está abaixo de cada vértice. Em seguida, refazemos esse processo para o segundo período, mas agora somando o custo de cada aresta com o custo obtido no passo anterior. Finalmente, o processo é refeito para o primeiro período. Dessa forma, o menor caminho, que representa a melhor solução para o problema, é dado pelas soluções B-G-P. A Figura 3.10b destaca as arestas pertencentes ao menor caminho. Note que, a solução A possui custo menor que a solução $B$, uma estratégia míope seguiria esse caminho e descartaria o caminho B. Entretanto, o menor caminho iniciando pela solução A possui custo 138 (A-C-H).

Como podemos perceber pela Figura 3.10, além de ser praticamente impossível gerar todas as soluções viáveis para cada período do problema, a complexidade computacional cresce exponencialmente em relação ao tamanho do problema, ou seja, a aplicação da programação dinâmica é limitada pela dimensão do problema, o que é conhecido como "maldição de dimensionalidade" ou "problema de dimensionalidade" [44], o que torna sua aplicação computacionalmente impraticável em problemas de médio e grande porte.

Motivados pela necessidade de tratar o problema de dimensionalidade, muitos trabalhos propõem estratégias para aproximações em programação dinâmica. Tais métodos são amplamente conhecidos como Programação Dinâmica Aproximada (PDA). A ênfase da PDA é conseguir lidar com o problema de dimensionalidade considerando aproximações para o problema global ou aproximações para a função objetivo do problema original, que devem ser computadas e armazenadas de forma eficiente. Diferentemente da programação dinâmica, o objetivo da PDA não é a otimização global do problema, mas sim encontrar uma solução de boa qualidade para o problema, dadas as limitações computacionais. Além disso, a PDA inclui vários métodos e variações, uma vez que nenhum dos métodos é considerado geral o suficiente para ser aplicável a todos os problemas. Por isso, a escolha de uma modelagem em PDA é altamente dependente do problema [43].

Baseando-se no grafo de transição da Figura 3.10, uma abordagem PDA analisa os dois primeiros vértices do primeiro período e uma estimativa de economia futura de cada vértice é feita para decidir qual caminho seguir (A ou B). A cada vértice é atribuído uma política associada a economia estimada futura para auxiliar na decisão. Após decidir o caminho a seguir, fixa-se o vértice escolhido e o processo é refeito para o próximo período. Esse processo é refeito até que todos os períodos sejam analisados. Ao final, as políticas de economia são recalculadas e a PDA é reiniciada, mas agora usando as novas políticas de economia.

Uma forma de se resolver o PCBMPSA, usando PDA, é resolver o problema considerando cada período de forma individual. Entretanto, como dito anteriormente, essa é uma abordagem gulosa que normalmente não encontra uma solução ótima global. Assim, com o objetivo de melhorar a seleção dos objetos em cada período e, consequentemente, conseguir uma solução global melhor, devemos saber o quanto podemos economizar no futuro ao selecionar um determinado conjunto de objetos no período $s<P$.

Seja $O_{s j}$ o objeto $j$ do período $s$. Perceba que ao utilizar um objeto $O_{s j}$, suas sobras aproveitáveis, caso existam, podem ser utilizadas no corte de itens futuros, isto é, ao comprar o objeto $O_{s j}$, podemos ter uma economia futura proporcional ao valor de suas sobras aproveitáveis, caso elas sejam utilizadas. 
Dessa forma, podemos considerar o custo artificial $\widetilde{C}_{s j}$ do objeto $O_{s j}$ como sendo

$$
\tilde{C}_{s j}=c_{s j}\left(H_{s j} W_{s j}-\left(\gamma_{s j}^{\mathrm{r}}+\gamma_{s j}^{\mathrm{t}}\right)\right)
$$

onde $\gamma_{s j}^{\mathrm{r}}$ e $\gamma_{s j}^{\mathrm{t}}$ são, respectivamente, as áreas aproveitáveis da direita e superior do objeto $O_{s j}$. Dessa forma, estamos sendo otimistas e dizendo que o custo do objeto $O_{s j}$ não é mais o seu custo por unidade de área vezes a sua área, mas vezes sua área de corte (vide Figura 2.7), em outras palavras, estamos supondo que suas sobras aproveitáveis serão inteiramente aproveitadas em algum período posterior. Lembrando que objetos não selecionados não possuem sobras aproveitáveis, ou seja, $\gamma_{s j}^{\mathrm{r}}=0$ e $\gamma_{s j}^{\mathrm{t}}=0$ se $u_{s j}=0$. Assim, a função objetivo no período $s, p \leq s<P$, pode ser dada por,

$$
\min \sum_{j=1}^{\bar{m}_{s}} c_{s j}\left(W_{s j} H_{s j} u_{s j}-\left(\gamma_{s j}^{\mathrm{r}}+\gamma_{s j}^{\mathrm{t}}\right)\right)
$$

Como exemplo, considere os dados de uma instância ilustrada pela Figura 3.11. Para definirmos qual conjunto de objetos deve ser selecionado no primeiro período, inicialmente, simulamos o empacotamento dos itens do período em diferentes conjuntos de objetos. Como existe um único item no período e este pode ser empacotado em qualquer um dos três objetos (considere, para este exemplo, que objetos não utilizados não são selecionados), teremos três conjuntos de objetos, cada um com um objeto. A Figura 3.12 mostra cada um dos objetos do primeiro período, com o item do período empacotado e as sobras aproveitáveis de cada objeto. O próximo passo, para definir qual conjunto de objetos devemos selecionar, é considerar as sobras aproveitáveis para calcular a economia futura que cada objeto pode gerar e escolher o conjunto de menor valor de acordo com o custo artificial dos objetos dado pela equação (3.1).

Inicialmente, supomos que a área das sobras aproveitáveis de cada objeto será totalmente utilizada no corte de itens futuros (suposição otimista), ou seja, supomos que a economia futura ao selecionar um objeto é igual ao valor das sobras aproveitáveis. Usando a equação (3.1) e supondo que as sobras aproveitáveis serão totalmente utilizadas, temos que $\widetilde{C}_{11}=231, \widetilde{C}_{12}=209, \widetilde{C}_{13}=312$, e, assim, o objeto $O_{12}$ é selecionado para o corte dos itens do primeiro período, já que possui o menor custo. Note que, sem considerarmos a economia dos objetos, ou seja, em uma estratégia gulosa, o objeto $O_{13}$ seria selecionado, uma vez que é o mais barato e consegue atender a ordem de trabalho dos itens do período.

Essa estratégia é repetida no segundo período para selecionar os objetos utilizados. No último período, ao invés de usarmos a estratégia PDA, usamos a abordagem gulosa, já que não temos informações dos itens das ordens de trabalho futuras. A Figura 3.13 ilustra a solução global encontrada, cujo custo total dos objetos utilizados é de 521.

Perceba que a sobra do objeto $O_{12}$ não foi totalmente utilizada ao final do horizonte de planejamento, embora inicialmente tenhamos suposto que a sobra aproveitável do objeto seria totalmente utilizada e que geraria uma economia futura no valor de 152. Entretanto, tal sobra foi utilizada para cortar três itens $\{7 \times 6,6 \times 5,6 \times 5\}$ que ocupam, aproximadamente, $67 \%$ da área da sobra aproveitável, o que mostra que nossa suposição otimista de economia do objeto $O_{12}$ estava equivocada, pois não 
utilizamos $100 \%$ da sobra aproveitável do objeto. Já a sobra do objeto $O_{21}$ não foi utilizada para cortar nenhum item, ou seja, $0 \%$ da sobra aproveitável foi de fato utilizada. Como nossa estimativa inicial de economia não se manteve, podemos agora iniciar o processo novamente, mas estimando que a economia gerada ao selecionar o objeto $O_{12}$ e o objeto $O_{21}$ será de, respectivamente, $67 \%$ e $0 \%$ do valor da sobra aproveitável.

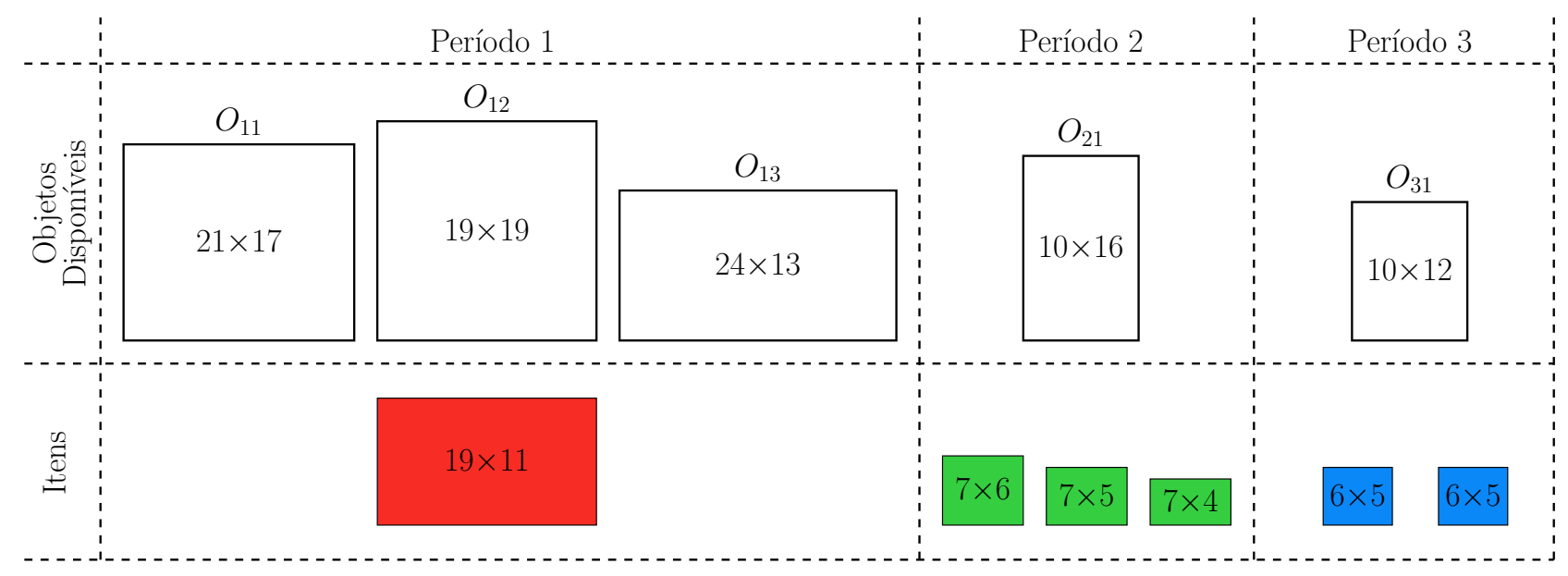

Figura 3.11: Instância para exemplificar a abordagem baseada em programação dinâmica aproximada utilizada neste trabalho. Suponha que $c_{s j}=1$, para $s=1, \ldots, 3, j=1, \ldots, m_{s}$.

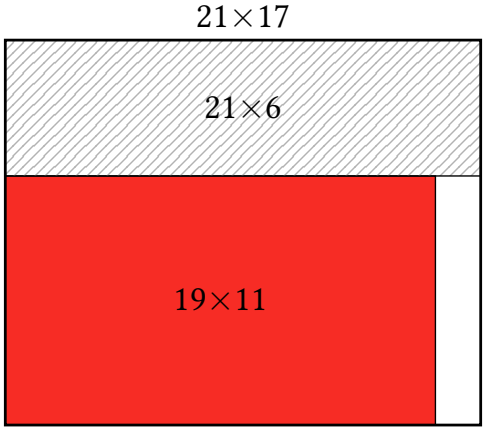

(a) Objeto $O_{11} \operatorname{com} \tilde{C}_{11}=231$
$19 \times 19$

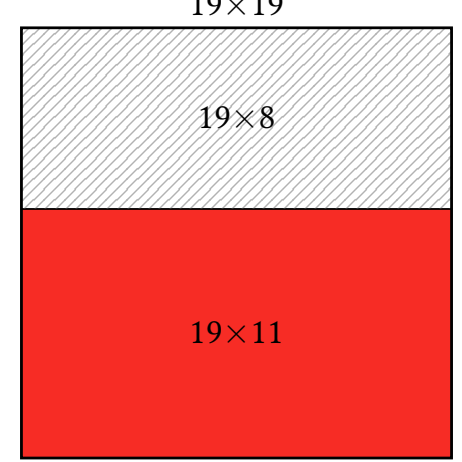

(b) Objeto $O_{12} \operatorname{com} \widetilde{C}_{12}=209$

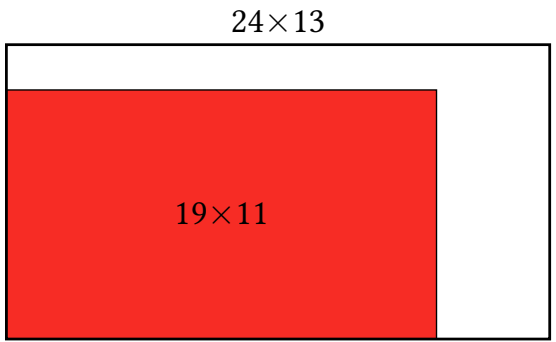

(c) Objeto $O_{13} \operatorname{com} \widetilde{C}_{13}=312$

Figura 3.12: Cálculo do custo artificial de cada objeto do primeiro período da instância ilustrada pela Figura 3.11, ou seja, o custo do objeto menos o valor de suas sobras aproveitáveis.

Sejam $\delta_{s j k}^{\mathrm{r}}$ e $\delta_{s j k}^{\mathrm{t}}$ a estimativa da proporção de utilização futura (ou fração de economia) da sobra aproveitável da direita e da sobra aproveitável superior, respectivamente, do objeto $j$ no período $s$ na iteração $k$, após o empacotamento de todos os itens do horizonte de planejamento. Considere também, $\delta_{\text {ini }}$ o valor inicial de $\delta_{s j 1}^{\mathrm{r}}$ e $\delta_{s j 1}^{\mathrm{t}}$, para $s=p, \ldots, P-1, j=1, \ldots, \bar{m}_{s}$. Não há itens futuros quando $s=P$, por isso não podemos calcular a estimativa da proporção de utilização futura de cada sobra, assim, $\delta_{P j k}^{\mathrm{r}}=0$ e $\delta_{P j k}^{\mathrm{t}}=0$, para $j=1, \ldots, \bar{m}_{P}$ e para toda iteração $k$. Além disso, caso um objeto não tenha sobra aproveitável da direita (quando $\gamma_{s j}^{\mathrm{r}}=0$ ) ou superior (quando $\gamma_{s j}^{\mathrm{t}}=0$ ), a estimativa da proporção de utilização futura referente a tal sobra é definida como zero. Ou seja, na iteração $k$ temos que 


$$
\left.\begin{array}{l}
\delta_{s j k}^{\mathrm{r}}= \begin{cases}\delta_{\text {ini }} & \text { se } k=1 \text { e } s \neq P \\
0 & \text { se }\left(k>1 \text { e } \gamma_{s j}^{\mathrm{r}}=0\right) \text { ou } s=P \\
\frac{A_{s j}^{\mathrm{r}}}{\gamma_{s j}^{\mathrm{r}}} & \text { se } k>1 \text { e } \gamma_{s j}^{\mathrm{r}} \neq 0 \text { e } s \neq P\end{cases} \\
\delta_{s j k}^{\mathrm{t}}= \begin{cases}\delta_{\text {ini }} & \text { se } k=1 \text { e } s \neq P \\
0 & \text { se }\left(k>1 \text { e } \gamma_{s j}^{\mathrm{t}}=0\right) \text { ou } s=P \\
\frac{A_{s j}^{\mathrm{t}}}{\gamma_{s j}^{\mathrm{t}}} & \text { se } k>1 \text { e } \gamma_{s j}^{\mathrm{t}} \neq 0 \text { e } s \neq P\end{cases}
\end{array}\right\} s=p, \ldots, P, j=1, \ldots, \bar{m}_{s},
$$

onde $A_{s j}^{\mathrm{r}}$ é a soma da área de todos os itens cortados na sobra da direita e $A_{s j}^{\mathrm{t}}$ é a soma da área de todos os itens cortados na sobra superior do objeto $j$ no período $s$. Para o exemplo anterior, supomos que $\delta_{\text {ini }}=1$ (suposição otimista). Dessa forma, na iteração $k$, o custo artificial $\widetilde{C}_{s j}$ do objeto $j$ no período $s$ se torna

$$
\tilde{C}_{s j}=c_{s j}\left(H_{s j} W_{s j}-\left(\delta_{s j k}^{\mathrm{r}} \gamma_{s j}^{\mathrm{r}}+\delta_{s j k}^{\mathrm{t}} \gamma_{s j}^{\mathrm{t}}\right)\right)
$$

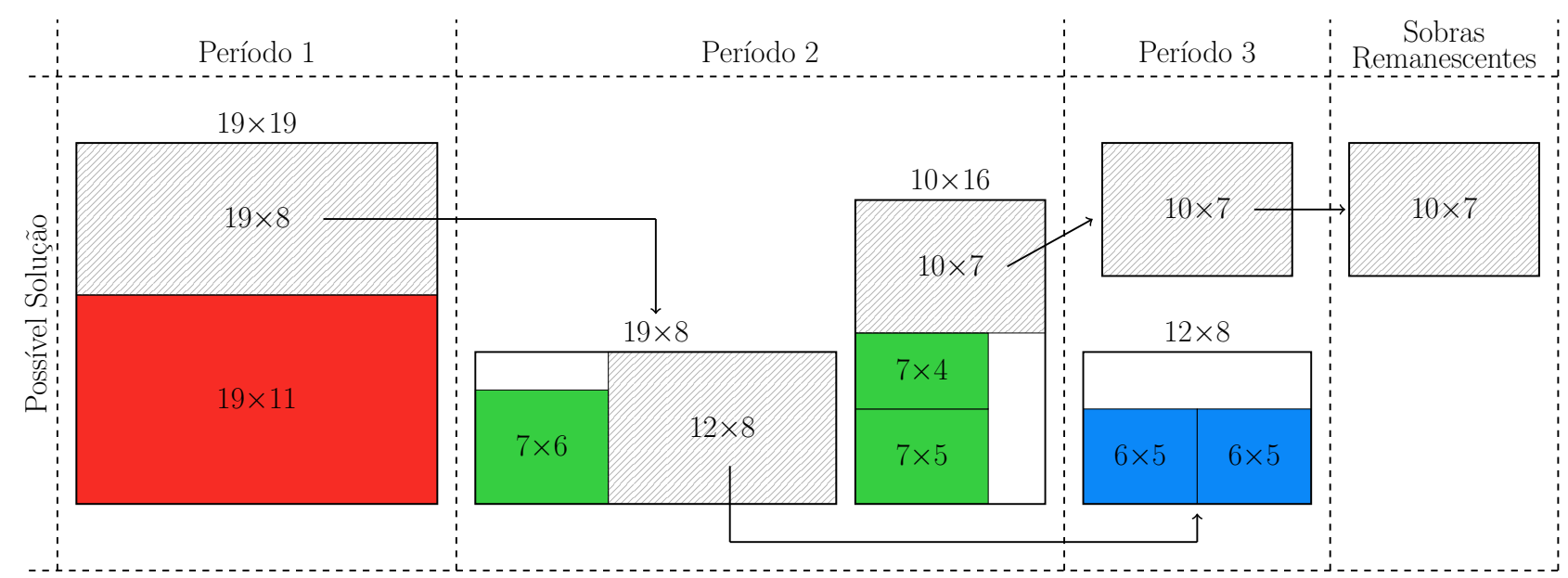

Figura 3.13: Solução encontrada ao utilizar a equação (3.1) para selecionar o conjunto de objetos utilizados e supondo, inicialmente, que as sobras aproveitáveis geradas serão totalmente utilizadas no corte de itens futuros. Dessa forma, chegamos a uma solução com custo global dos objetos cortados igual a 521. Para simplificar, apenas os objetos de entrada utilizados são mostrados.

Continuando com o exemplo anterior e considerando a equação (3.4), na segunda iteração, o novo custo do objeto $O_{12}$ é dado por $\widetilde{C}_{12}=1 \times(19 \times 19-(1,0 \times(0 \times 0)+0,67 \times(19 \times 8)))=259,16$, os outros objetos não foram selecionados na iteração anterior, portanto, o valor da fração de economia das sobras desses objetos permanecem com o mesmo valor da iteração anterior. Dessa forma, $\widetilde{C}_{11}=231$, $\widetilde{C}_{12}=259,16, \widetilde{C}_{13}=312$. Assim, o objeto $O_{11}$ é selecionado no primeiro período, gerando uma sobra de $21 \times 6$.

No segundo período, temos o objeto de entrada $O_{21}$ e a sobra gerada pelo objeto $O_{11}$. Note que o objeto $O_{21}$ pode gerar, no máximo, uma sobra aproveitável (a superior) e, da iteração anterior, tal sobra 
não foi utilizada no corte, ou seja, ela não gerou economia futura. Logo, o custo artificial do objeto $O_{21}$ é $\widetilde{C}_{21}=160$. Tal valor é maior que o custo artificial da sobra do objeto $O_{11}, 1 \times(21 \times 6)=126$ que pode empacotar os três itens no período. Portanto, no segundo período apenas a sobra do objeto $O_{11}$ é utilizada. A Figura 3.14 ilustra essa solução, cujo custo global é de 477.

Novamente, a sobra aproveitável não foi totalmente utilizada, ou seja, devemos repetir o processo considerando que a economia da sobra do objeto $O_{11}$ é de $83 \%$ do seu valor (área vezes o custo por unidade de área), o que gera os seguintes custos $\widetilde{C}_{11}=252,4, \widetilde{C}_{12}=283,5, \widetilde{C}_{13}=312$. Como o objeto $O_{11}$ possui o menor custo benefício e não houve outras alterações na porcentagem das economias dos objetos, não precisamos repetir o processo e a solução retornada é a de custo 477, que coincide com a solução ótima para a instância.

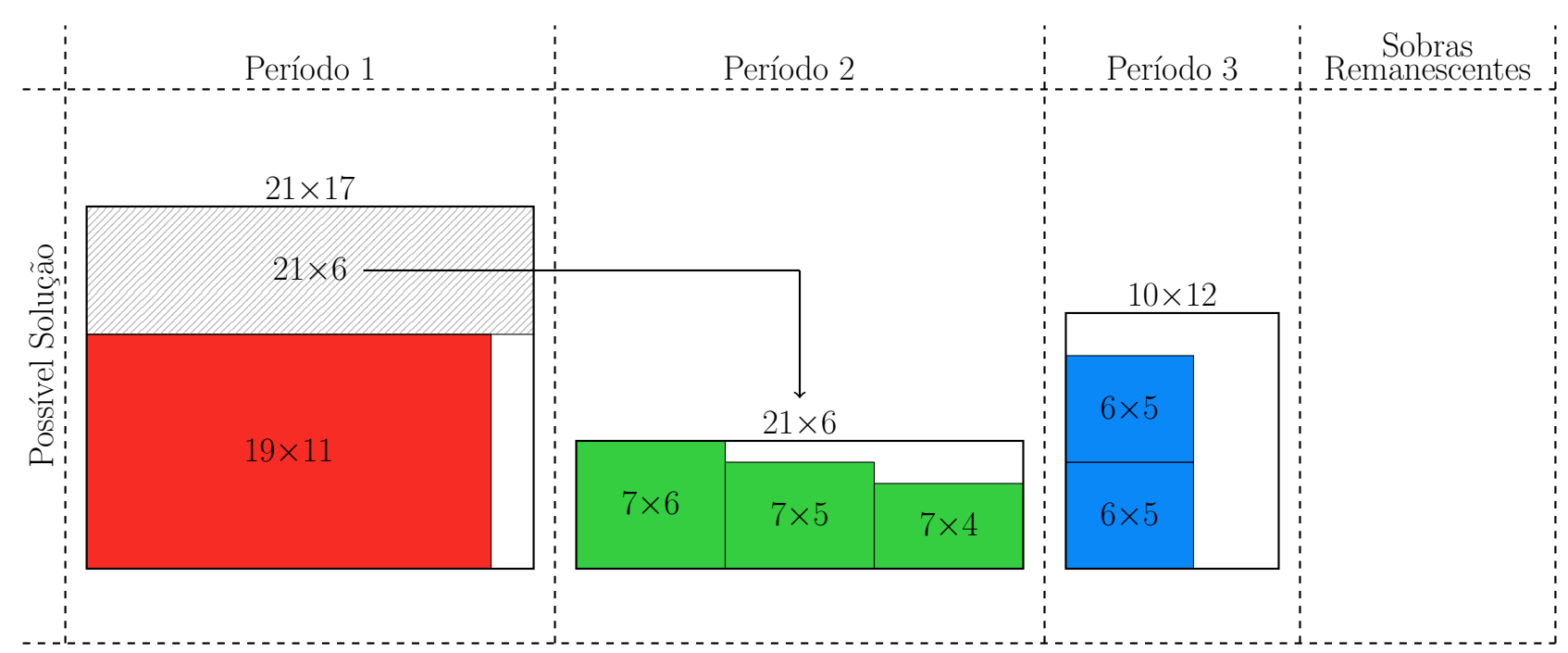

Figura 3.14: Solução encontrada após ser calculada a proporção da utilização das sobras de cada objeto e considerando a equação (3.4) para selecionar o conjunto de objetos comprados. Dessa forma, chegamos a uma solução com custo global dos objetos utilizados igual a 477.

Note que os valores de $\delta_{s j k}^{\mathrm{r}}$ e $\delta_{s j k}^{\mathrm{t}}$ podem ser zero, como aconteceu com o objeto $O_{21}$ na primeira iteração do exemplo. Nesse caso, ao considerar a equação (3.4), o valor das sobras será anulado, fazendo com que o modelo não necessariamente maximize o valor das sobras aproveitáveis de tais objetos. Para resolver esse problema, podemos fazer com que o modelo escolha a solução de menor custo dado por (3.2) e, dentre todas as soluções de menor custo, escolha a que maximize o valor das sobras, ou seja, temos, novamente, um modelo em dois níveis.

Como a função objetivo do problema do nível superior e do nível inferior possuem limitantes superiores e inferiores triviais, podemos reformular o modelo de dois níveis como um modelo MIP. Perceba que o valor das sobras aproveitáveis, do período $s$, admite valores maiores ou iguais a zero e estritamente menores que $\sum_{j=1}^{\bar{m}_{s}} c_{s j} W_{s j} H_{s j}$. Assim, na iteração $k$, a função objetivo no período $s$ é dada por:

$$
\left(\sum_{j=1}^{\bar{m}_{s}} c_{s j} W_{s j} H_{s j}\right)\left(\sum_{j=1}^{\widehat{m}_{s}} c_{s j}\left(W_{s j} H_{p j} u_{s j}-\left(\delta_{s j k}^{\mathrm{r}} \gamma_{s j}^{\mathrm{r}}+\delta_{s j k}^{\mathrm{t}} \gamma_{s j}^{\mathrm{t}}\right)\right)\right)-\sum_{j=1}^{\bar{m}_{s}} c_{s j}\left(\gamma_{s j}^{\mathrm{r}}+\gamma_{s j}^{\mathrm{t}}\right),
$$


onde, $\widehat{m}_{s}=\bar{m}_{s}$, se $s<P$, e $\widehat{m}_{s}=m_{s}$, se $s=P$. Note que, quando $s=P$, a função objetivo (3.5) se torna

$$
\left(\sum_{j=1}^{\bar{m}_{s}} c_{s j} W_{s j} H_{s j}\right)\left(\sum_{j=1}^{m_{s}} c_{s j} W_{s j} H_{s j} u_{s j}\right)-\sum_{j=1}^{\bar{m}_{s}} c_{s j}\left(\gamma_{s j}^{\mathrm{r}}+\gamma_{s j}^{\mathrm{t}}\right) .
$$

que coincide com a função objetivo do problema de corte com sobras aproveitáveis de um único período.

Dessa forma, o modelo MIP para o problema de empacotamento com sobras aproveitáveis usado pela PDA, no período $s$, chamado de agora em diante de $\tilde{\mathcal{M}}_{s, s}$, usa as variáveis $u_{s j} \in\{0,1\}(j=$ $\left.1, \ldots, \bar{m}_{s}\right)$ para indicar se o objeto $j$ no período $s$ é utilizado ou não; $v_{s i j} \in\{0,1\}\left(i=1, \ldots, n_{s}, j=\right.$ $\left.1, \ldots, \bar{m}_{s}\right)$ para definir em qual objeto cada item deve estar; $\left(x_{s i}, y_{s i}\right) \in \mathbb{R}^{2}\left(i=1, \ldots, n_{s}\right)$ para definir a posição dos itens dentro dos objetos; $\pi_{s i i^{\prime}} \in\{0,1\}\left(i=1, \ldots, n_{s}, i^{\prime}=i+1, \ldots, n_{s}\right)$ e $\tau_{s i i^{\prime}} \in\{0,1\}\left(q_{s}=1, \ldots, \tilde{n}_{s}, i=o_{s q}+1, \ldots, o_{s q}+z_{s q}, i^{\prime}=i+1, \ldots, n_{s}\right)$ para modelar e impedir a sobreposição dos itens; $\eta_{s j} \in\{0,1\}\left(j=1, \ldots, \bar{m}_{s}\right)$ para selecionar o tipo de corte guilhotinado (Caso A ou Caso B) para gerar as sobras de cada objeto; $\gamma_{s j}^{\mathrm{r}}$ e $\gamma_{s j}^{\mathrm{t}} \in \mathbb{R}\left(j=1, \ldots, \bar{m}_{s}\right)$ para representar a área da sobra da direita e da sobra superior, respectivamente, do tipo de corte com melhor sobra aproveitável dos objetos; $\theta_{s j \ell}^{\mathrm{r}} \in\{0,1\}\left(j=1, \ldots, \bar{m}_{s}, \ell=1, \ldots,\left\lfloor\log _{2}\left(W_{s j}\right)\right\rfloor+1\right)$ para modelar a representação binária da largura máxima da sobra da direita dos objetos e $\theta_{s j \ell}^{\mathrm{t}} \in\{0,1\}(j=$ $\left.1, \ldots, \bar{m}_{s}, \ell=1, \ldots,\left\lfloor\log _{2}\left(H_{s j}\right)\right\rfloor+1\right)$ para modelar a representação binária da altura máxima da sobra superior dos objetos; $\omega_{s j \ell}^{\mathrm{rt}}, \omega_{s j \ell}^{\mathrm{Wt}} \in \mathbb{R}\left(j=1, \ldots, \bar{m}_{s}, \ell=1, \ldots,\left\lfloor\log _{2}\left(H_{s j}\right)\right\rfloor+1\right)$ e $\omega_{s j \ell}^{\mathrm{rH}} \in \mathbb{R}(j=$ $\left.1, \ldots, \bar{m}_{s}, \ell=1, \ldots,\left\lfloor\log _{2}\left(W_{s j}\right)\right\rfloor+1\right)$ para modelar o produto que aparece quando é calculada a área das sobras de cada objeto; $\alpha_{s j}^{\mathrm{t}}, \alpha_{s j}^{\mathrm{r}}, \beta_{s j}^{\mathrm{t}}$ e $\beta_{s j}^{\mathrm{r}} \in \mathbb{R},\left(j=1, \ldots, \bar{m}_{s}\right)$ para representar a área das sobras aproveitáveis do objeto $j$ no período $s$; e $\bar{\alpha}_{s i j}^{\mathrm{t}}, \bar{\alpha}_{s i j}^{\mathrm{r}}, \bar{\beta}_{s i j}^{\mathrm{t}}$ e $\bar{\beta}_{s i j}^{\mathrm{r}} \in\{0,1\},\left(i=1, \ldots, d, j=1, \ldots, \bar{m}_{s}\right)$ para indicar se a sobra do objeto $j$ no período $s$ comporta algum item do catálogo. Assim, a formulação do problema, no período $s$, consiste em minimizar (3.5) nas variáveis $u_{s j}, v_{s i j}, x_{s i}, y_{s i}, \pi_{s i i^{\prime}}, \tau_{s i i^{\prime}}, \eta_{s j}$, $\gamma_{s j}^{\mathrm{r}}, \gamma_{s j}^{\mathrm{t}}, \theta_{s j \ell}^{\mathrm{r}}, \theta_{s j \ell}^{\mathrm{t}}, \omega_{s j \ell}^{\mathrm{rt}}, \omega_{s j \ell}^{\mathrm{Wt}}, \omega_{s j \ell}^{\mathrm{rH}}, \alpha_{s j}^{\mathrm{t}}, \alpha_{s j}^{\mathrm{r}}, \beta_{s j}^{\mathrm{t}}, \beta_{s j}^{\mathrm{r}}, \bar{\alpha}_{s i j}^{\mathrm{t}}, \bar{\alpha}_{s i j}^{\mathrm{r}}, \bar{\beta}_{s i j}^{\mathrm{t}}$ e $\bar{\beta}_{s i j}^{r}$ sujeito a $(2.19,2.20,2.25,2.32$, $2.33,2.34,2.37,2.41,2.45,2.46,2.48$ e 2.49$)$. No modelo, toda aparição de $\bar{W}_{s j}$ e $\bar{H}_{s j}$ deve ser substituída por $W_{s j}$ e $H_{s j}$, respectivamente, para $j=1, \ldots, \bar{m}_{s}$, toda aparição de $r_{s j}$ e $t_{s j}$ deve ser substituída por suas representações binárias (2.39) e (2.40), respectivamente, e os produtos $r_{s j} t_{s j}, \bar{W}_{s j} t_{s j}$ e $r_{s j} \bar{H}_{s j}$ devem ser substituídos por suas respectivas representações MIP, dadas por (2.42), (2.43) e (2.44).

Note que quando $s<P$ o modelo $\tilde{\mathcal{M}}_{s, s}$ possui as mesmas restrições do modelo $\mathcal{M}_{s, s}^{2}$, mas com função objetivo diferente, e $\tilde{\mathcal{M}}_{s, s}$ é equivalente ao modelo $\mathcal{M}_{s, s}^{2}$ quando $s=P$.

\subsubsection{Atualização das estimativas de utilização das sobras}

Na equação (3.3) apresentamos uma alternativa para calcular as estimativas de proporção de utilização futura das sobras, usada em nossa abordagem em PDA. Outra possibilidade, como apresentado em [44], é atualizar os valores de $\delta_{s j k}^{\mathrm{r}}$ e $\delta_{s j k}^{\mathrm{t}}$ observando seus valores da iteração anterior (valores antigos) e indo uma certa porcentagem (digamos $\sigma$ ) em direção aos valores sugeridos pela iteração atual (novos valores). Por exemplo, se $\delta_{s j 1}^{\mathrm{r}}=1,0$ e, ao final do horizonte de planejamento, a proporção de utilização da sobra da direita do objeto $O_{s j}$ é de 0,5 , usando a equação (3.3), temos que $\delta_{s j 2}^{\text {r }}=0,5$. Adotando 
a segunda alternativa e supondo que $\sigma=50 \%$, temos que $\delta_{s j 2}^{\mathrm{r}}=0,75$, ou seja, o valor da estimativa de utilização da sobra irá $\sigma=50 \%$ em direção ao novo valor. Além disso, para assegurarmos a convergência do método, consideramos $[\sigma]^{k-1}(\sigma$ elevado a $k-1)$. Dessa forma, permitimos mudanças maiores nas primeiras iterações nos valores de $\delta_{s j k}^{\mathrm{r}}$ e $\delta_{s j k}^{\mathrm{t}}$ e garantimos a convergência das estimativas de utilização das sobras e, assim, a convergência do método. Portanto, para $s=p, \ldots, P$, e $j=$ $1, \ldots, \bar{m}_{s}$, definimos:

$$
\begin{aligned}
& \delta_{s j k}^{\mathrm{r}}= \begin{cases}\delta_{\text {ini }} & \text { se } k=1 \text { e } s \neq P \\
\left(1-[\sigma]^{k-1}\right) \delta_{s, j, k-1}^{\mathrm{r}} & \text { se } k>1 \text { e } \gamma_{s j}^{\mathrm{r}}=0 \text { e } s \neq P \\
\left(1-[\sigma]^{k-1}\right) \delta_{s, j, k-1}^{\mathrm{r}}+[\sigma]^{k-1}\left(\frac{A_{s j}^{\mathrm{r}}}{\gamma_{s j}^{\mathrm{r}}}\right) & \text { se } k>1 \text { e } \gamma_{s j}^{\mathrm{r}} \neq 0 \text { e } s \neq P \\
0 & \text { se } s=P\end{cases} \\
& \delta_{s j k}^{\mathrm{t}}= \begin{cases}\delta_{\text {ini }} & \text { se } k=1 \text { e } s \neq P \\
\left(1-[\sigma]^{k-1}\right) \delta_{s, j, k-1}^{\mathrm{t}} & \text { se } k>1 \text { e } \gamma_{s j}^{\mathrm{t}}=0 \text { e } s \neq P \\
\left(1-[\sigma]^{k-1}\right) \delta_{s, j, k-1}^{\mathrm{t}}+[\sigma]^{k-1}\left(\frac{A_{s j}^{\mathrm{t}}}{\gamma_{s j}^{\mathrm{t}}}\right) & \text { se } k>1 \text { e } \gamma_{s j}^{\mathrm{t}} \neq 0 \text { e } s \neq P \\
0 & \text { se } s=P\end{cases}
\end{aligned}
$$

onde $\sigma$ é uma constante tal que $0<\sigma \leq 1$. Note que se $\sigma=1$, os valores de $\delta_{s j k}^{\mathrm{r}}$ e $\delta_{s j k}^{\mathrm{t}}$ são iguais à fração de utilização da sobra da direita e sobra superior, respectivamente, como definido na equação (3.3).

\subsubsection{Critério de parada}

O critério de parada adotado no algoritmo proposto é como segue. Na iteração $k$, se todos os valores das estimativas de proporção de utilização futura das sobras forem próximos dos valores de alguma outra iteração $k^{\prime}<k$, então o algoritmo é encerrado. Formalmente, o algoritmo termina na iteração $k$ se:

$$
\begin{aligned}
& \left|\delta_{s j k}^{\mathrm{r}}-\delta_{s j k^{\prime}}^{\mathrm{r}}\right| \leq \epsilon \delta_{s j k}^{\mathrm{r}} \\
& \left|\delta_{s j k}^{\mathrm{t}}-\delta_{s j k^{\prime}}^{\mathrm{t}}\right| \leq \epsilon \delta_{s j k}^{\mathrm{t}}
\end{aligned} \quad s=p, \ldots, P-1, j=1, \ldots, \bar{m}_{s}, k^{\prime}=1, \ldots, k-1,
$$

onde $\epsilon>0$ é uma tolerância dada.

Perceba que se as estimativas de proporção de utilização das sobras na iteração $k$ forem muito parecidas com as de alguma iteração $k^{\prime}$ anterior então, muito provavelmente, uma nova iteração devolveria, novamente, a solução encontrada na iteração $k^{\prime}$.

\subsubsection{Pseudocódigo do algoritmo proposto}

O Algoritmo 3.4 apresenta o pseudocódigo da estratégia PDA proposta para o PCBMPSA. O algoritmo recebe como entrada, além dos parâmetros vistos anteriormente, outros três parâmetros: 
- $\delta_{\text {ini: }}$ fração inicial da estimativa de economia das sobras aproveitáveis;

- $\sigma$ : tamanho do passo usado em (3.6);

- $\epsilon$ : parâmetro de tolerância de erro usado em (3.7)

Inicialmente o algoritmo define a quantidade $\bar{m}_{s}$ de objetos que podem estar disponíveis em cada período $s$ e as estimativas de proporção de utilização das sobras futuras dos $\bar{m}_{s}$ objetos de cada período $s$ são inicializadas. Além disso, o algoritmo utiliza a variável $S^{*}$ para armazenar a melhor solução encontrada durante sua execução. Em seguida, o algoritmo começa a fase de calibração das estimativas de proporção de utilização das sobras futuras, que é executada até que o critério de parada (3.7) seja satisfeito. Em cada iteração $k$, o algoritmo resolve o modelo $\tilde{\mathcal{M}}_{s, s}$, para cada período $s=p, \ldots, P$. Após a resolução do modelo para o período $s$, na linha 8 , calculamos o valor da largura da sobra da direita e altura da sobra superior de cada um dos $\bar{m}_{s}$ objetos. Caso não estejamos no último período, as sobras aproveitáveis devem estar disponíveis no próximo período como novos objetos e utilizando os valores das variáveis encontradas na resolução de $\tilde{\mathcal{M}}_{s, s}$ (com auxílio do Algoritmo 3.2), determinamos as dimensões e valor por unidade de área da sobra da direita e da sobra superior de cada um dos $\bar{m}_{s}$ objetos do período $s$. Na linha 11 atribuímos as dimensões e custo por unidade de área de cada sobra em sua posição correta no período $s+1$. Note que no período $s+1$, os primeiros $m_{s+1}$ objetos são os objetos de entrada (disponíveis para serem comprados) e as próximas posições $\left(m_{s+1}+1\right.$ até $\left.\bar{m}_{s+1}\right)$ são objetos originados de sobras dos períodos anteriores.

Após o algoritmo resolver $\tilde{\mathcal{M}}_{s, s}$ para $s=p, \ldots, P$, computamos o valor global da solução encontrada na iteração $k$ e o armazenamos em $S_{k}$. Se tal solução for melhor que a solução encontrada nas iterações anteriores, armazenada em $S^{*}$, atualizamos o valor de $S^{*}$ e armazenamos os valores das variáveis necessárias para construirmos a solução global do problema. Em seguida, calculamos a estimativa da proporção que será utilizada no futuro de cada sobra. Para isso, precisamos saber a área utilizada pela sobra da direita e pela sobra superior dos objetos de cada período. Assim, sejam $A_{s j}^{\mathrm{r}}$ e $A_{s j}^{\mathrm{t}}$ a soma da área dos itens cortados, respectivamente, na sobra da direita e na sobra superior no objeto $j$ do período $s$; e seja $A_{s j}$ a área total utilizada no objeto $j$ no período $s$, ou seja, $A_{s j}=\sum_{i=1}^{n_{s}} v_{s i j} w_{s i} h_{s i}+A_{s j}^{\mathrm{r}}+A_{s j}^{\mathrm{t}}$. Inicialmente, na linha 30, determinamos a soma da área dos itens que foram atribuídos a cada objeto originado de uma sobra. Entre as linhas 31 e 49, calculamos as estimativas de proporção de utilização das sobras futuras dos $\bar{m}_{s}$ objetos de cada período $s$ seguindo a equação (3.6).

Note que no último período só podemos ter itens cortados na área de corte do objeto $j$ (uma vez que não temos itens futuros) e, assim, as sobras desse objeto não são utilizadas para cortar itens das ordens de trabalho. Por outro lado, nos períodos $s<P$ as sobras de cada objeto podem ter sido utilizadas para cortar itens de ordens de trabalho futuras. Como as sobras da direita dos objetos do período $s$ são posicionadas uma após a outra, em sequência, após os $m_{s+1}$ objetos do período $s+1$ e as sobras superiores são posicionadas uma após a outra após as sobras da direita, no período $s<P$, a área utilizada pela sobra da direita do objeto $j$ corresponde a área total utilizada pelo objeto $m_{s+1}+j$ do período $s+1$ e a sobra superior corresponde ao objeto $m_{s+1}+\bar{m}_{s}+j$ do período $s+1$. Dessa forma, quando $s<P, A_{s j}^{\mathrm{r}}=A_{s+1, m_{s+1}+j}, A_{s j}^{\mathrm{t}}=A_{s+1, m_{s+1}+\bar{m}_{s}+j}$ e usamos esses valores para 
atualizar a área total $\left(A_{s j}\right)$ utilizada pelo objeto $j$ no período $s$ (linha 46), que corresponde a área dos itens cortados na área de corte do objeto, somado com a área dos itens cortados na sobra da direita e na sobra superior do objeto.

Quando o critério de parada (3.7) for satisfeito, o algoritmo retorna a melhor solução encontrada durante sua execução juntamente com as variáveis necessárias para construirmos tal solução. Vale destacar que quanto menor o valor de $\sigma$, menor será o número de iterações que o Algoritmo 3.4 gastará para convergir e quanto menor o valor de $\delta_{\text {ini }}$, menor será o número de objetos que têm a chance de serem utilizados para o corte de itens em cada período. Isso porque os objetos terão seu custo reduzido de um pequeno valor (correspondente ao valor de suas sobras multiplicado pela estimativa de proporção de utilização futura das sobras, que é pequena), o que faz com que seja menos vantajoso utilizar objetos de maior custo.

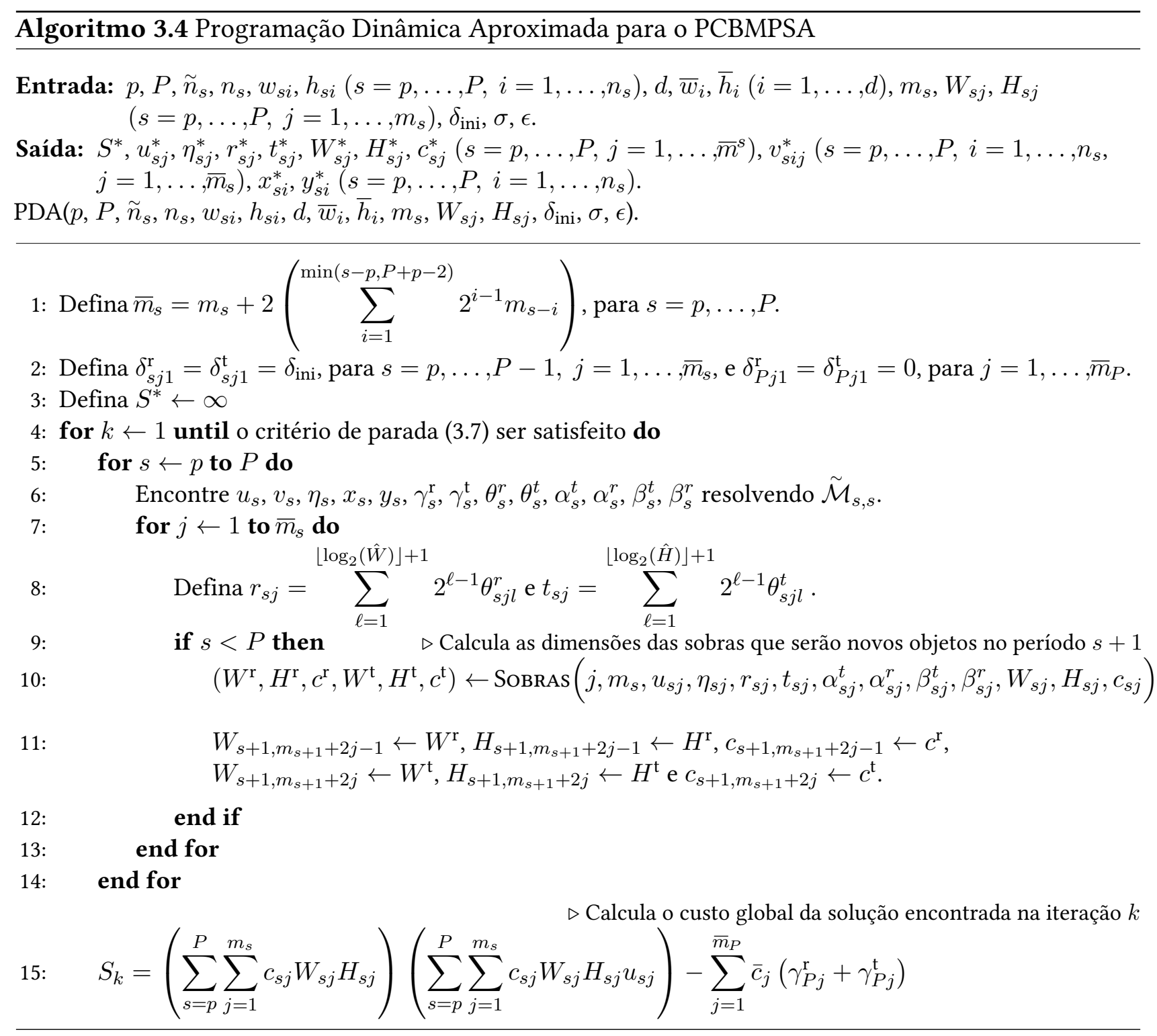




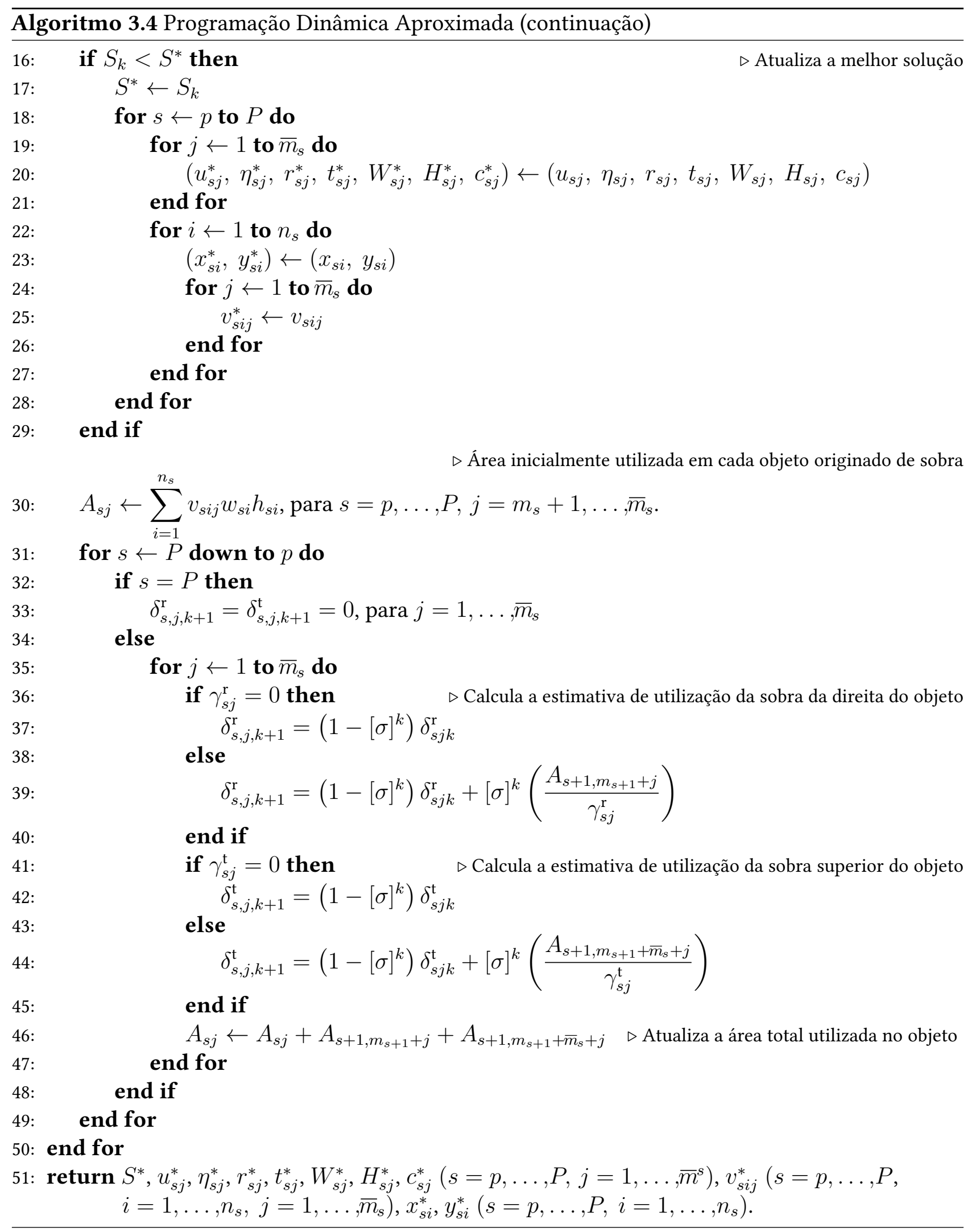




\subsection{Experimentos computacionais}

Nesta seção, apresentamos alguns experimentos computacionais ilustrativos conduzidos para avaliar e comparar os resultados obtidos pelas abordagens heurísticas propostas para resolver o PCBMPSA. Todos os algoritmos e modelos foram implementados em C++ e compilados com GNU g++ versão 5.4 com a opção -03 ativada. No caso dos modelos, utilizamos o IBM ILOG CPLEX versão 12.6.3, sendo implementada via ILOG Concert Technology. Com relação aos parâmetros do CPLEX, definimos o valor da variável EpGap para 0. Também pedimos para que o CPLEX armazene a árvore Branch \& Bound em disco caso ela exceda 12000MB (definimos NodeFileInd como 3 e WorkMem como 12000). Todos os experimentos foram realizados em uma máquina Intel Core i7-3770 3.40GHz, com 16 GB de memória RAM e com Sistema Operacional Linux.

Para os experimentos numéricos dessa seção, geramos 30 instâncias, baseadas em [3], mostradas na Tabela A.1. As instâncias são divididas em três grupos (cada um com 10 instâncias). Esses são definidos pelo número de períodos do horizonte de planejamento (4, 8 ou 12). Em todos os experimentos consideramos o modelo $\mathcal{M}_{p, P}^{2}$ (o qual permite a compra de objetos que não são usados no corte de itens, mas só permite que sejam feitos cortes em objetos com itens), por ser a opção mais natural. Além disso, também consideramos que uma sobra gerada a partir de um objeto de entrada do período $s$ pode ficar disponível para todos os períodos seguintes, ou seja, definimos $\xi=P+p-2$.

Como métrica para avaliar a qualidade das soluções obtidas pelos algoritmos heurísticos propostos, usamos a medida do Desvio Percentual Relativo (RPD em inglês Relative Percentage Deviation) dado por:

$$
R P D=\frac{f_{\text {metodo }}-f_{\text {melhor }}}{f_{\text {melhor }}} \times 100 \%,
$$

onde $f_{\text {metodo }}$ é a solução obtida por um dado método e $f_{\text {melhor }}$ é a melhor solução obtida entre os métodos comparados.

Nosso primeiro conjunto de experimentos visa comparar os resultados obtidos pela PDA com diferentes valores de $\sigma$ e $\delta_{\text {ini. }}$. Consideramos a PDA com os seguintes parâmetros: $\sigma \in\{0,1 ; 0,5 ; 0,8 ; 0,9\}$, $\delta_{\text {ini }} \in\{0,10 ; 0,20 ; 0,30 ; 0,40 ; 0,50 ; 0,60 ; 0,70 ; 0,80 ; 0,90 ; 1,00\}$. Os resultados são resumidos no gráfico da Figura 3.15. O valor de $f_{\text {melhor }}$, usado no cálculo do $R P D$, corresponde a melhor solução encontrada pela PDA, de cada instância, para as diferentes combinações de $\sigma$ e $\delta_{\text {ini }}$ testadas. Em relação aos experimentos, definimos o tempo de relógio limite do CPLEX para 10 minutos (atribuímos ao parâmetro TiLim o valor 600). Isso significa que o tempo limite para o CPLEX resolver o modelo com os dados de um único período, em cada iteração, é de 10 minutos. Quanto ao critério de parada da PDA, definimos $\epsilon=0,01$ na equação (3.7).

O gráfico da Figura 3.15 mostra que a PDA consegue resultados semelhantes para os diferentes valores de $\sigma$ e $\delta_{\text {ini. }}$. Entretanto, podemos notar que quando $\sigma \in\{0,8 ; 0,9\}$ e $\delta_{\text {ini }} \in\{0,80 ; 0,90\}$ temos uma menor variabilidade dos $R P D$ 's e esses estão mais próximos de zero, indicando que, com esses valores de $\sigma$ e $\delta_{\text {ini }}$, a PDA obtém melhores soluções. Para auxiliar na escolha da combinação de valores de $\sigma$ e $\delta_{\text {ini }}$ usadas pela PDA, os gráficos das Figuras 3.16a e 3.16b mostram, respectivamente, o RPD e o tempo médio da PDA. Pela Figura 3.16a é fácil perceber que o RPD médio tende a diminuir à medida que aumentamos o valor de $\delta_{\text {ini }}$, exceto para $\delta_{\text {ini }}=1,00$, quando o RPD médio aumenta 
novamente. Além disso, a PDA com $\sigma \in\{0,8 ; 0,9\}$ obtém em um RPD médio melhor do que a PDA com $\sigma \in\{0,1 ; 0,5\}$. Isso acontece porque o método com $\sigma \in\{0,1 ; 0,5\}$ irá convergir mais rapidamente (fazendo um menor número de iterações) ao ser comparado com outros valores de $\sigma$. Para essas instâncias a PDA que conseguiu o melhor RPD médio (7,43\%) foi com $\sigma=0,9$ e $\delta_{\text {ini }}=0,90$. Por essas razões, definimos $\sigma=0,9$ e $\delta_{\text {ini }}=0,90$ para serem usados pela PDA nesses experimentos.

Em relação ao tempo médio gasto pela PDA, a Figura 3.16b mostra que aumentar o valor de $\sigma$ e $\delta_{\text {ini }}$ implica em aumentar o tempo médio gasto pelo método. Observe também que o tempo médio gasto quando $\delta_{\text {ini }}=1,00$ é muito superior quando comparado com os outros valores de $\delta_{\text {ini }}$, isso acontece porque na primeira iteração do método o modelo irá selecionar o máximo de objetos possíveis (inclusive objetos que não foram usados para cortar itens) em cada período, ou seja, a cada período o número de objetos disponíveis irá aumentar, fazendo com que o modelo fique cada vez mais difícil e demorado de se resolver.

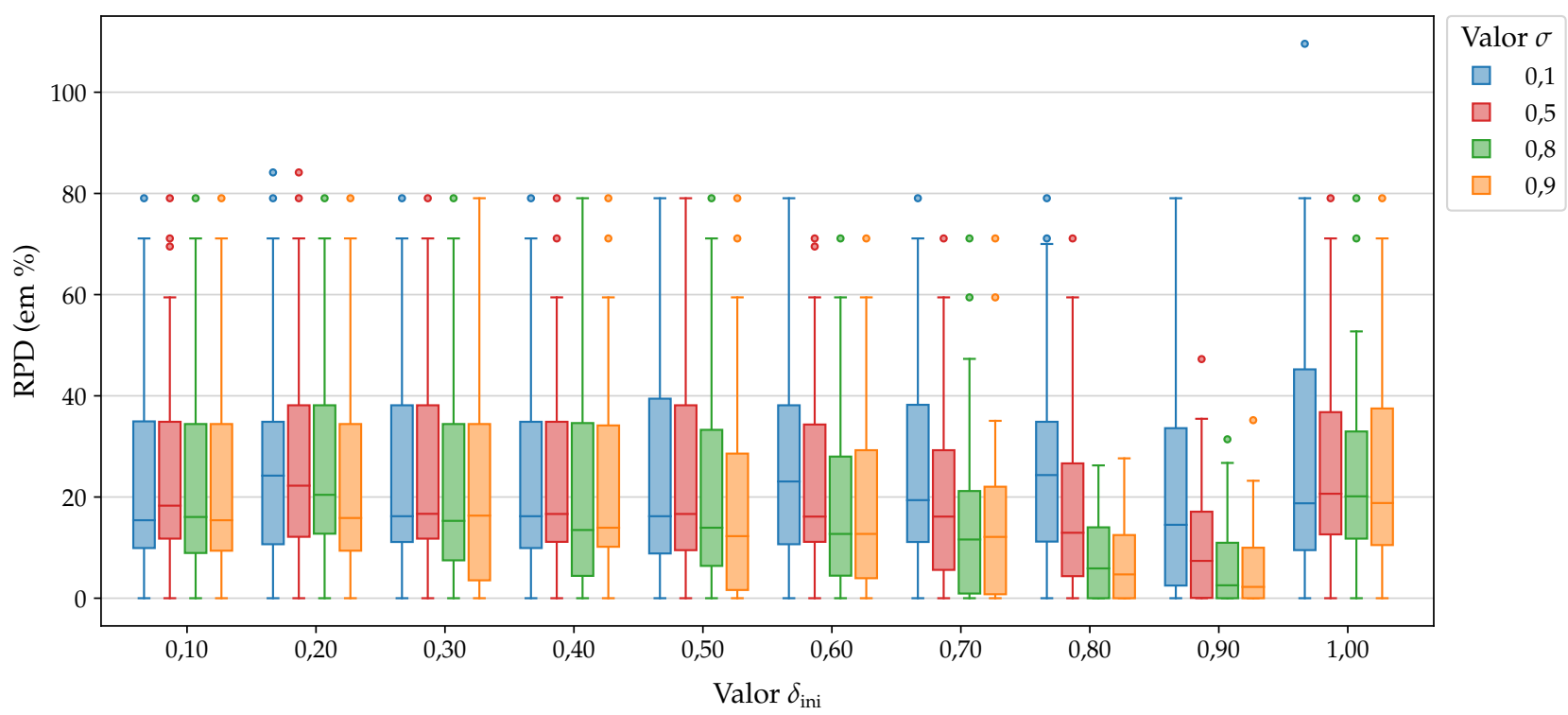

Figura 3.15: RPD para a PDA considerando diferentes valores de $\sigma$ e $\delta_{\text {ini }}$

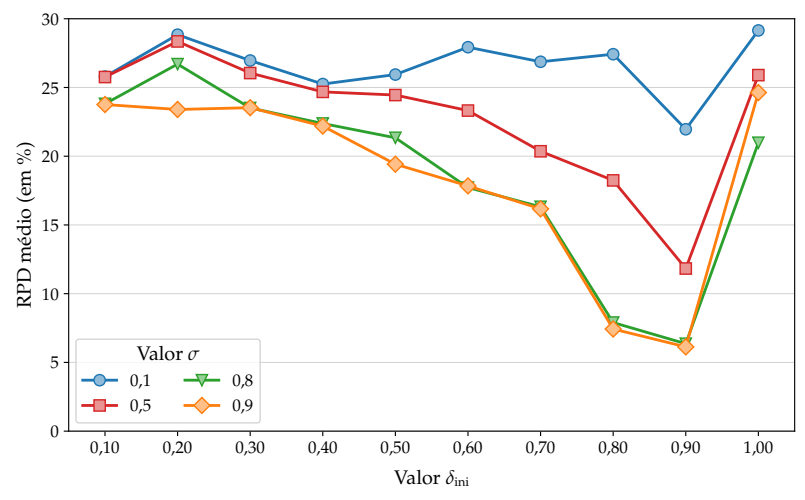

(a) RPD médio

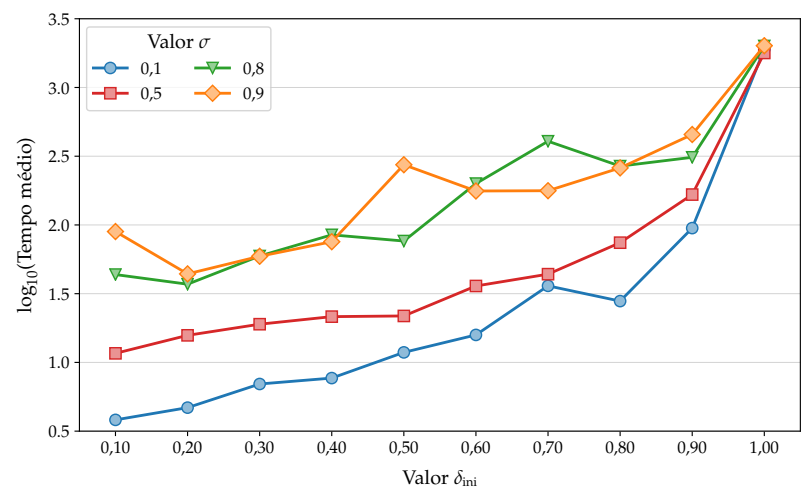

(b) Tempo médio

Figura 3.16: RPD e tempo médios da PDA para cada combinação de valor de $\delta_{\text {ini }}$ e $\sigma$. 
O próximo conjunto de experimentos, mostrado na Tabela 3.1, visa comparar a solução obtida pelo horizonte rolante, pelo relax-and-fix e pela PDA. Para o horizonte rolante e relax-and-fix consideramos a versão mais simples, isto é, a janela de decisão com tamanho $\Delta=1$ e sem sobreposição, ou seja, $\Phi=0$ (em $\operatorname{HR}(1-0)$ e $\mathrm{RF}(1-0)$ o primeiro número se refere ao valor de $\Delta$ e o segundo ao valor de $\Phi)$. Para cada instância e para cada método, a coluna "Solução" informa o valor da função objetivo (2.47) encontrada pelo método correspondente; a coluna " $R P D$ " diz o quão distante (em porcentagem) a solução encontrada pelo método está da melhor solução obtida (mostrada na última coluna) entre os três métodos considerados na tabela (as entradas marcadas com “-” indicam que o RPD vale 0); a coluna "Tempo" mostra a quantidade de tempo, em segundos, que levou até a solução ser obtida. A última coluna mostra a melhor solução de cada instância encontrada pelos métodos considerados na tabela. Nesses experimentos, definimos o tempo de relógio limite do CPLEX, para resolver cada subproblema, em 10 minutos e para o critério de parada da PDA, definimos $\epsilon=0,01$.

Tabela 3.1: Resultado numérico da PDA e das versões mais simples do horizonte rolante e relax-andfix.

\begin{tabular}{|c|c|c|c|c|c|c|c|c|c|c|}
\hline \multirow{2}{*}{ Inst. } & \multicolumn{3}{|c|}{$\operatorname{HR}(1-0)$} & \multicolumn{3}{|c|}{$\mathrm{RF}(1-0)$} & \multicolumn{3}{|c|}{ PDA } & \multirow{2}{*}{$\begin{array}{l}\text { Melhor } \\
\text { Solução }\end{array}$} \\
\hline & Solução & RPD & Tempo & Solução & RPD & Tempo & Solução & RPD & Tempo & \\
\hline 1 & 726.545 & - & 1,13 & 726.553 & 0,00 & 1,08 & 726.563 & 0,00 & 5,73 & 726.545 \\
\hline 2 & 534.143 & - & 1,04 & 534.146 & 0,00 & 1,00 & 534.198 & 0,01 & 6,97 & 534.143 \\
\hline 3 & 1.182 .322 & - & 1,09 & 1.182 .322 & - & 1,23 & 1.182 .322 & - & 9,46 & 1.182 .322 \\
\hline 4 & 3.692 .593 & 26,56 & 1,08 & 3.692 .593 & 26,56 & 1,12 & 2.917 .570 & - & 7,99 & 2.917 .570 \\
\hline 5 & 2.091 .636 & - & 1,26 & 2.091 .636 & - & 1,37 & 2.091 .662 & 0,00 & 15,86 & 2.091 .636 \\
\hline 6 & 34.478 .101 & 29,03 & 3,04 & 34.478 .101 & 29,03 & 3,06 & 26.721 .158 & - & 70,90 & 26.721 .158 \\
\hline 7 & 24.558 .187 & 45,32 & 1,83 & 24.558 .187 & 45,32 & 1,78 & 16.899 .377 & - & 13,44 & 16.899 .377 \\
\hline 8 & 45.935 .944 & - & 2,04 & 45.935 .944 & - & 2,54 & 45.935 .984 & 0,00 & 46,17 & 45.935 .944 \\
\hline 9 & 57.823 .489 & 3,97 & 1,65 & 57.823 .479 & 3,97 & 3,06 & 55.614 .336 & - & 78,11 & 55.614 .336 \\
\hline 10 & 34.242 .404 & 59,45 & 1,18 & 34.242 .404 & 59,45 & 1,67 & 21.474 .954 & - & 31,79 & 21.474 .954 \\
\hline 11 & 5.579 .595 & 26,49 & 1,97 & 5.579 .595 & 26,49 & 5,84 & 4.411 .006 & - & 25,60 & 4.411 .006 \\
\hline 12 & 6.809 .101 & 12,00 & 2,71 & 8.143 .645 & 33,95 & 7,35 & 6.079 .436 & - & 21,14 & 6.079 .436 \\
\hline 13 & 5.672 .446 & 59,45 & 3,48 & 4.752 .483 & 33,59 & 7,29 & 3.557 .438 & - & 17,66 & 3.557 .438 \\
\hline 14 & 5.312 .502 & 41,50 & 2,65 & 5.312 .502 & 41,50 & 6,12 & 3.754 .328 & - & 18,56 & 3.754 .328 \\
\hline 15 & 6.876 .271 & 56,25 & 2,01 & 6.876 .271 & 56,25 & 4,21 & 4.400 .755 & - & 9,72 & 4.400 .755 \\
\hline 16 & 136.287 .697 & 37,85 & 22,37 & 111.604 .323 & 12,89 & 24,50 & 98.864 .853 & - & 76,06 & 98.864 .853 \\
\hline 17 & 105.386 .567 & 12,74 & 5,26 & 126.590 .571 & 35,43 & 41,04 & 93.474 .193 & - & 204,96 & 93.474 .193 \\
\hline 18 & 83.691 .201 & 10,92 & 5,28 & 86.296 .896 & 14,37 & 214,39 & 75.451 .522 & - & 95,74 & 75.451 .522 \\
\hline 19 & 76.464 .680 & 27,03 & 11,82 & 89.961 .210 & 49,45 & 16,82 & 60.195 .048 & - & 68,69 & 60.195 .048 \\
\hline 20 & 122.868 .459 & 14,97 & 17,06 & 122.868 .358 & 14,97 & 270,57 & 106.867 .140 & - & 94,45 & 106.867 .140 \\
\hline 21 & 6.981 .269 & 28,33 & 4,51 & 6.981 .277 & 28,33 & 80,20 & 5.440 .012 & - & 52,16 & 5.440 .012 \\
\hline 22 & 6.553 .629 & - & 6,63 & 11.153 .364 & 70,19 & 64,76 & 7.202 .654 & 9,90 & 135,70 & 6.553 .629 \\
\hline 23 & 9.071 .588 & 35,83 & 7,02 & 7.775 .748 & 16,42 & 116,66 & 6.678 .827 & - & 26,94 & 6.678 .827 \\
\hline 24 & 12.393 .930 & 57,97 & 13,95 & 9.422 .570 & 20,09 & 107,73 & 7.845 .955 & - & 43,56 & 7.845 .955 \\
\hline 25 & 6.895 .729 & - & 4,73 & 8.065 .173 & 16,96 & 154,84 & 7.182 .102 & 4,15 & 43,93 & 6.895 .729 \\
\hline 26 & 263.506 .990 & 2,84 & 439,51 & 293.874 .032 & 14,69 & 901,92 & 256.237 .067 & - & 429,70 & 256.237 .067 \\
\hline 27 & 169.554 .092 & 14,69 & 12,50 & 175.908 .183 & 18,99 & 463,29 & 147.836 .095 & - & 321,24 & 147.836 .095 \\
\hline 28 & 269.377 .442 & 0,63 & 50,43 & 269.377 .465 & 0,63 & 925,28 & 267.693 .154 & - & 846,69 & 267.693 .154 \\
\hline 29 & 272.580 .083 & 42,30 & 722,88 & 244.248 .475 & 27,51 & $2.411,08$ & 191.550 .586 & - & $4.470,35$ & 191.550 .586 \\
\hline 30 & 179.276 .748 & 31,46 & 45,97 & 174.175 .755 & 27,72 & $1.234,43$ & 136.371 .356 & - & 807,95 & 136.371 .356 \\
\hline \multicolumn{2}{|c|}{ Média (1-10) } & 16,43 & 1,54 & & 16,43 & 1,79 & & 0,00 & 28,64 & \\
\hline \multicolumn{2}{|c|}{ Média (11-20) } & 29,92 & 7,46 & & 31,89 & 59,81 & & - & 63,26 & \\
\hline \multicolumn{2}{|c|}{ Média (21-30) } & 21,40 & 130,81 & & 24,15 & 646,02 & & 1,41 & 717,82 & \\
\hline \multicolumn{2}{|c|}{ Média (1-30) } & 22,59 & 46,60 & & 24,16 & 235,87 & & 0,47 & 269,91 & \\
\hline
\end{tabular}

Comparando os três métodos, podemos notar claramente que a PDA obteve as melhores soluções em praticamente todas as instâncias. Enquanto a PDA encontrou 24 melhores soluções ( $R P D$ igual a 0), o HR(1 - 0) encontrou 7 e o RF(1 - 0) 3. Apenas em duas instâncias (22 e 25) a PDA encontrou uma solução com $R P D$ maior que $1 \%$, a melhor solução dessas duas instâncias foi obtida pelo HR(1 - 0). 
O RPD médio obtido pela PDA foi de 0,47 , enquanto no $\mathrm{HR}(1-0)$ e no $\mathrm{RF}(1-0)$ o $R P D$ médio foi cerca de $23 \%$ e $24 \%$, respectivamente. É interessante notar que o $R F(1-0)$ obteve um RPD médio um pouco maior que o $H R(1-0)$. Apenas em 8 instâncias $(9,13,16,20,23,24,29$ e 30) o $R F(1-0)$ foi melhor que o HR(1 - 0). Provavelmente, o baixo desempenho do relax-and-fix se dá por conta das relaxações feitas na formulação que, provavelmente, o forçaram a fazer escolhas não muito boas.

$\mathrm{O}$ método $\mathrm{HR}(1-0)$ foi o método com menor tempo médio, em seguida temos o $\mathrm{RF}(1-0)$ e o mais demorado foi a PDA. Com exceção das três últimas instâncias, em todas as outras a PDA gastou menos de 10 minutos para encontrar uma solução, isso mostra que, para essas instâncias, a resolução de nenhum subproblema, em todas as iterações, consumiu o tempo limite para chegar na solução ótima do subproblema. No HR $(1-0)$ apenas a instância 29 gastou mais de 10 minutos; para tal instância, analisando os logs gerados pelo CPLEX, pelo menos um subproblema não chegou à solução ótima no tempo limite. O mesmo acontece para as instâncias 29 e 30 para o $\mathrm{RF}(1-0)$.

Com o objetivo de melhorar as soluções obtidas pelos métodos horizonte rolante e relax-andfix, podemos aumentar o tamanho da janela de decisão $(\Delta)$ e também permitir sobreposição de períodos. Entretanto, os 10 minutos de tempo de relógio limite para a resolução de cada subproblema se tornam insuficientes, isto é, para muitas instâncias o CPLEX pode não conseguir sequer encontrar uma solução inteira para um subproblema em 10 minutos. Assim, para os próximos experimentos definimos o tempo de relógio limite do CPLEX para 2 horas, ou seja, em cada janela de decisão o CPLEX tem 2 horas para reportar uma solução inteira. Caso seja atingido o tempo limite, a melhor solução inteira obtida é considerada.

A Tabela 3.2 mostra as soluções encontradas pelo horizonte rolante variando o tamanho da janela de decisão e o número de períodos sobrepostos. Testamos diferentes versões do horizonte rolante: $\operatorname{HR}(2-0), \operatorname{HR}(2-1), \operatorname{HR}(3-0), \operatorname{HR}(3-1)$ e $\operatorname{HR}(3-2)$, onde o primeiro número representa o tamanho da janela de decisão $(\Delta)$ e o segundo o número de períodos sobrepostos $(\Phi)$. É fácil notar que a medida que aumentamos o valor de $\Delta$, a resolução do método se torna cada vez mais cara computacionalmente, por isso, testamos o horizonte rolante com $\Delta \leq 3$. Observe que se $\Delta=4$ temos a formulação exata completa para as instâncias pequenas (numeradas de 1 a 10) que, como vimos no capítulo anterior, a resolução dos modelos pelo CPLEX exige um tempo considerável.

Comparando as cinco versões do horizonte rolante notamos que com $\Delta=3$ e $\Phi=2$ foi possível encontrar, na maioria das instâncias, as melhores soluções. Para as instâncias de 1 a 13, nenhuma outra versão conseguiu encontrar soluções melhores que HR(3 - 2). Entretanto, como esperado, esta é a versão do horizonte rolante que, em média, consumiu mais tempo. É importante notar que permitir sobreposição de período(s) não garante que a solução sempre será melhor que a solução da versão sem sobreposição. Por exemplo, $\operatorname{HR}(2-0)$ encontrou solução melhor que $\operatorname{HR}(2-1)$ nas instâncias 8 , 15, 21 , 26, 27, 28 e 29; e HR(3 - 0) encontrou solução melhor que HR(3 - 1) e HR(3 - 2) nas instâncias $14,15,18,23,24,27$ e 28 . O mesmo acontece quando o valor de $\Delta$ é aumentado, não há garantias de que a solução sempre será melhorada, como podemos ver na instância 21 em que a melhor solução é obtida por HR(2 - 0). Para as instâncias de 21 a 30, a versão HR(3 - 0) obteve um $R P D$ médio um pouco maior que $\operatorname{HR}(3-2)$, mas o tempo médio gasto por $\mathrm{HR}(3-0)$ foi quase 3 vezes menor.

Em relação ao tempo gasto para a solução ser encontrada, $\operatorname{HR}(2-0)$ possui o menor tempo médio 
(aproximadamente 2 horas) enquanto $\mathrm{HR}(3-2)$ possui o maior (quase 7 horas), mas a qualidade das soluções obtidas por $\mathrm{HR}(2-0)$, normalmente, são piores que as encontradas por $\operatorname{HR}(3-2)$. Podemos notar que HR(2 - 0) encontrou as melhores soluções em menor tempo, como mostra o gráfico da Figura 3.17, para as instâncias 3, 5, 21 e 22. Em geral, quanto maior o valor de $\Delta$ e menor a diferença entre $\Delta$ e $\Phi$, melhores serão as soluções encontradas pelo método, mas o tempo para obter tais soluções será maior.

A Tabela 3.3 mostra as soluções encontradas pelas diferentes versões do relax-and-fix. Os resultados foram similares aos do horizonte rolante. A versão que obteve, na grande maioria, as melhores soluções foi o $\mathrm{RF}(3-2)$, também foi a versão que obteve as melhores soluções para as instâncias 1 a 13. Entretanto, $R F(3-2)$ é a versão, em média, que mais demora para encontrar as soluções. As versões $\mathrm{RF}(3-0)$ e $\mathrm{RF}(3-2)$ foram as que apresentam o maior número de melhores soluções, enquanto o $\mathrm{RF}(3-2)$ obteve 20 melhores soluções, $\mathrm{RF}(3-0)$ encontrou 13. Podemos notar também que a versão $\mathrm{RF}(3-0)$ foi a que obteve, em média, as melhores soluções para as instâncias de tamanho médio (instâncias 11 a 20), tendo um $R P D$ médio de 3,46. Para essas instâncias o $\mathrm{RF}(3-0)$ foi, em média, quase 3 vezes mais rápido que $\mathrm{RF}(3-2)$. Para as instâncias 21 a 30 o $\mathrm{RF}(3-0)$ teve um $R P D$ médio maior apenas que o $\mathrm{RF}(3-2)$, mas só não foi, em média, mais rápido que o $\mathrm{RF}(2-0)$, sendo aproximadamente 2 vezes mais rápido que o $\mathrm{RF}(3-2)$.

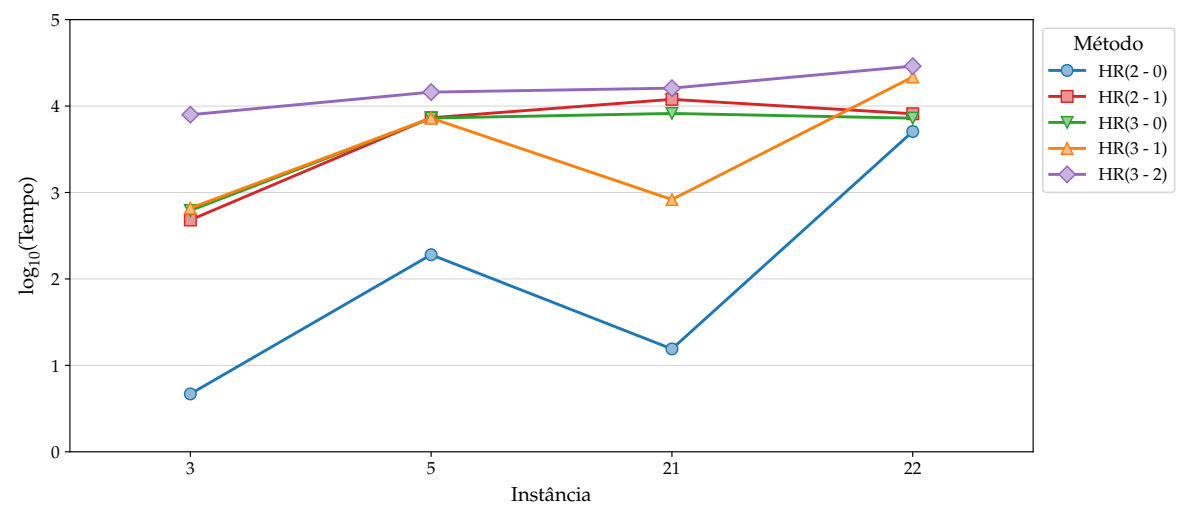

Figura 3.17: Tempo gasto pelas diferentes versões do horizonte rolante para obter a solução de quatro instâncias. Nessas instâncias, as melhores soluções foram obtidas por $\operatorname{HR}(2-0)$, que foi a versão que consumiu menos tempo. 
Tabela 3.2: Resultado numérico das diferentes versões do Horizonte Rolante para as instâncias do PCBMPSA.

\begin{tabular}{|c|c|c|c|c|c|c|c|c|c|c|c|c|c|c|c|c|}
\hline \multirow{2}{*}{ Inst. } & \multicolumn{3}{|c|}{$\operatorname{HR}(2-0)$} & \multicolumn{3}{|c|}{$\operatorname{HR}(2-1)$} & \multicolumn{3}{|c|}{$\operatorname{HR}(3-0)$} & \multicolumn{3}{|c|}{$\operatorname{HR}(3-1)$} & \multicolumn{3}{|c|}{$\mathrm{HR}(3-2)$} & \multirow{2}{*}{$\begin{array}{l}\text { Melhor } \\
\text { Solução }\end{array}$} \\
\hline & Solução & RPD & Tempo & Solução & RPD & Tempo & Solução & RPD & Tempo & Solução & RPD & Tempo & Solução & RPD & Tempo & \\
\hline 1 & 726.545 & 9,30 & 1,68 & 726.545 & 9,30 & 1,75 & 664.729 & - & 3,50 & 664.729 & - & 3,70 & 664.729 & - & 4,21 & 664.729 \\
\hline 2 & 534.143 & 0,00 & 2,29 & 534.146 & 0,00 & 2,52 & 534.135 & - & 6,87 & 534.135 & - & 7,46 & 534.135 & - & 9,77 & 534.135 \\
\hline 3 & 1.182 .322 & & 4,67 & 1.182 .322 & & 481,22 & 1.182 .322 & - & 619,24 & 1.182 .322 & - & 659,56 & 1.182 .322 & - & $7.939,06$ & 1.182 .322 \\
\hline 4 & 3.117 .169 & 44,44 & 23,76 & 3.117 .169 & 44,44 & 12,95 & 3.692 .593 & 71,10 & 2,12 & 3.117 .169 & 44,44 & 65,52 & 2.158 .129 & - & 330,89 & 2.158 .129 \\
\hline 5 & 2.091 .636 & - & 190,66 & 2.091 .636 & & $7.323,16$ & 2.091 .636 & . & $7.263,50$ & 2.091 .636 & & $7.256,89$ & 2.091 .636 & - & $14.505,18$ & 2.091 .636 \\
\hline 6 & 34.478 .073 & 29,03 & $7.235,22$ & 34.478 .081 & 29,03 & $9.841,50$ & 35.389 .333 & 32,44 & 144,94 & 30.956 .540 & 15,85 & $7.382,86$ & 26.720 .887 & - & $7.364,47$ & 26.720 .887 \\
\hline 7 & 22.209 .335 & - & $7.239,14$ & 22.209 .335 & - & $7.263,66$ & 24.558 .187 & 10,58 & 15,20 & 22.209 .335 & - & $7.287,12$ & 22.209 .335 & - & $7.272,18$ & 22.209 .335 \\
\hline 8 & 45.935 .944 & 22,78 & $7.224,82$ & 48.864 .400 & 30,61 & $13.118,59$ & 55.872 .952 & 49,34 & $7.219,99$ & 48.864 .400 & 30,61 & $14.469,08$ & 37.413 .879 & - & $14.450,68$ & 37.413 .879 \\
\hline 9 & 55.613 .386 & 21,16 & $7.378,69$ & 53.106 .600 & 15,70 & $11.185,33$ & 50.616 .467 & 10,28 & $7.211,70$ & 48.406 .461 & 5,46 & $13.859,42$ & 45.899 .670 & - & $14.422,81$ & 45.899 .670 \\
\hline 10 & 27.873 .171 & 0,00 & 149,17 & 27.873 .171 & 0,00 & 399,76 & 34.242 .341 & 22,85 & 14,77 & 27.873 .155 & & $2.568,84$ & 27.873 .155 & - & $7.243,35$ & 27.873 .155 \\
\hline 11 & 5.401 .763 & 0,28 & 12,92 & 5.401 .755 & 0,28 & 436,41 & 5.665 .967 & 5,19 & 160,38 & 5.401 .755 & 0,28 & $7.479,91$ & 5.386 .504 & - & $18.607,18$ & 5.386 .504 \\
\hline 12 & 8.143 .635 & 31,26 & $9.119,05$ & 8.143 .635 & 31,26 & $16.604,11$ & 6.725 .685 & 8,40 & 5,97 & 7.538 .927 & 21,51 & 80,80 & 6.204 .385 & - & 32,64 & 6.204 .385 \\
\hline 13 & 4.752 .478 & 32,98 & $7.259,83$ & 4.276 .074 & 19,65 & 24,58 & 4.760 .690 & 33,21 & $14.483,99$ & 4.703 .192 & 31,60 & $14.469,63$ & 3.573 .767 & - & $17.699,57$ & 3.573 .767 \\
\hline 14 & 4.644 .672 & 47,11 & $1.352,50$ & 4.644 .672 & 47,11 & $7.327,26$ & 3.157 .296 & - & $7.812,25$ & 3.157 .302 & 0,00 & $14.943,69$ & 4.644 .672 & 47,11 & $22.749,00$ & 3.157 .296 \\
\hline 15 & 5.929 .694 & 34,75 & 5,79 & 5.955 .699 & 35,34 & 33,32 & 4.400 .595 & - & 537,72 & 5.929 .689 & 34,75 & 339,06 & 4.400 .599 & 0,00 & 566,08 & 4.400 .595 \\
\hline 16 & 113.607 .793 & 18,55 & $7.283,56$ & 113.607 .752 & 18,55 & $19.660,44$ & 99.275 .793 & 3,59 & 82,63 & 95.833 .801 & - & $16.144,18$ & 95.833 .848 & 0,00 & $28.927,78$ & 95.833 .801 \\
\hline 17 & 123.637 .816 & 32,49 & $15.201,29$ & 93.321 .043 & - & $15.236,26$ & 96.502 .913 & 3,41 & $14.516,43$ & 96.502 .919 & 3,41 & $7.439,22$ & 96.502 .913 & 3,41 & $23.370,25$ & 93.321 .043 \\
\hline 18 & 83.691 .214 & 10,92 & $7.346,80$ & 75.451 .476 & - & $8.114,77$ & 75.451 .483 & 0,00 & $8.710,04$ & 86.930 .811 & 15,21 & $20.625,73$ & 78.691 .081 & 4,29 & $28.675,68$ & 75.451 .476 \\
\hline 19 & 89.961 .188 & 54,68 & $9.923,58$ & 71.631 .263 & 23,16 & $30.180,83$ & 100.288 .402 & 72,43 & $14.719,30$ & 74.246 .063 & 27,66 & $14.592,19$ & 58.161 .163 & - & $27.480,34$ & 58.161 .163 \\
\hline 20 & 109.844 .495 & 0,61 & $5.065,53$ & 109.844 .491 & 0,61 & $12.006,08$ & 109.844 .501 & 0,61 & $7.881,74$ & 109.176 .578 & & $14.451,33$ & 109.176 .588 & 0,00 & $29.095,91$ & 109.176.578 \\
\hline 21 & 5.719 .743 & - & 15,50 & 6.695 .632 & 17,06 & $11.960,79$ & 5.898 .205 & 3,12 & $8.216,26$ & 6.415 .952 & 12,17 & 829,36 & 5.898 .210 & 3,12 & $16.114,89$ & 5.719 .743 \\
\hline 22 & 6.719 .085 & - & $5.079,21$ & 6.719 .085 & - & $8.150,67$ & 9.015 .767 & 34,18 & $7.243,48$ & 6.719 .085 & - & $21.707,66$ & 6.719 .085 & - & $28.977,93$ & 6.719 .085 \\
\hline 23 & 7.584.938 & 17,34 & 27,63 & 7.115 .903 & 10,09 & $7.223,97$ & 6.464 .000 & - & $1.109,05$ & 7.871 .150 & 21,77 & $14.140,41$ & 7.871 .148 & 21,77 & $31.485,15$ & 6.464 .000 \\
\hline 24 & 8.202 .192 & 24,94 & $4.758,57$ & 8.202 .190 & 24,94 & $8.394,79$ & 6.564 .910 & - & $9.345,98$ & 8.202 .190 & 24,94 & $20.544,70$ & 8.202 .190 & 24,94 & $29.090,40$ & 6.564 .910 \\
\hline 25 & 6.895 .706 & 12,41 & 192,56 & 6.134 .326 & - & $8.079,56$ & 6.298 .799 & 2,68 & $15.666,31$ & 6.743 .426 & 9,93 & $27.700,71$ & 6.134 .329 & 0,00 & $46.113,14$ & 6.134 .326 \\
\hline 26 & 259.008 .669 & 10,28 & $18.642,76$ & 267.885 .440 & 14,06 & $40.985,98$ & 253.706 .316 & 8,02 & $21.776,46$ & 269.411 .872 & 14,71 & $36.284,61$ & 234.867.219 & - & $58.124,94$ & 234.867 .219 \\
\hline 27 & 140.691 .260 & 0,00 & $24.809,81$ & 145.070 .977 & 3,11 & $34.060,47$ & 140.691 .236 & - & $28.995,91$ & 158.030 .442 & 12,32 & $37.439,96$ & 142.091 .383 & 1,00 & $68.767,96$ & 140.691 .236 \\
\hline 28 & 292.881 .404 & 11,84 & $23.035,73$ & 313.376 .839 & 19,67 & $57.436,37$ & 261.876 .893 & - & $28.891,37$ & 272.746 .439 & 4,15 & $43.385,88$ & 280.407 .250 & 7,08 & $72.186,72$ & 261.876 .893 \\
\hline 29 & 235.240 .882 & 12,81 & $22.208,86$ & 238.705 .339 & 14,47 & $65.164,28$ & 209.950 .422 & 0,68 & $28.898,90$ & 222.306 .948 & 6,61 & $39.938,17$ & 208.526 .094 & - & $60.416,20$ & 208.526 .094 \\
\hline 30 & 166.266 .158 & 14,70 & $36.774,80$ & 153.191 .188 & 5,68 & $52.299,81$ & 167.008 .723 & 15,21 & $28.894,99$ & 158.679 .462 & 9,47 & $43.365,01$ & 144.958 .772 & - & $71.630,59$ & 144.958 .772 \\
\hline \multicolumn{2}{|c|}{ Média (1-10) } & 12,67 & 45,01 & & 12,91 & $4.963,05$ & & 19,66 & 0,18 & & 9,64 & 05 & & - & $7.354,26$ & \\
\hline \multirow{2}{*}{\multicolumn{2}{|c|}{$\begin{array}{l}\text { Média (11-20) } \\
\text { Média }(21-30)\end{array}$}} & 26,36 & $6.257,08$ & & 17,60 & $10.962,41$ & & 12,68 & $6.891,05$ & & 13,44 & $11.056,57$ & & 5,48 & $19.720,44$ & \\
\hline & & 10,43 & $13.554,54$ & & 10,91 & $29.375,67$ & & 6,39 & $17.903,87$ & & 11,61 & $28.533,65$ & & 5,79 & $48.290,79$ & \\
\hline \multicolumn{2}{|c|}{ Média (1-30) } & 16,49 & $7.585,55$ & & 13,80 & $15.100,37$ & & 12,91 & $9.015,03$ & & 11,56 & $14.982,09$ & & 3,76 & $25.121,83$ & \\
\hline
\end{tabular}


Tabela 3.3: Resultado numérico para as instâncias do PCBMPSA usando as diferentes versões do Relax-and-Fix.

\begin{tabular}{|c|c|c|c|c|c|c|c|c|c|c|c|c|c|c|c|c|}
\hline \multirow{2}{*}{ Inst. } & \multicolumn{3}{|c|}{$\mathrm{RF}(2-0)$} & \multicolumn{3}{|c|}{$\mathrm{RF}(2-1)$} & \multicolumn{3}{|c|}{$\mathrm{RF}(3-0)$} & \multicolumn{3}{|c|}{$\mathrm{RF}(3-1)$} & \multicolumn{3}{|c|}{$\mathrm{RF}(3-2)$} & \multirow{2}{*}{$\begin{array}{l}\text { Melhor } \\
\text { Solução }\end{array}$} \\
\hline & Solução & RPD & Tempo & Solução & RPD & Tempo & Solução & RPD & Tempo & Solução & RPD & Tempo & Solução & RPD & Tempo & \\
\hline 1 & 726.545 & 9,30 & 1,49 & 726.545 & 9,30 & 1,91 & 664.729 & - & 1,92 & 664.729 & - & 1,94 & 664.729 & - & 1,31 & 664.729 \\
\hline 2 & 534.135 & - & 1,49 & 534.135 & - & 2,24 & 534.135 & - & 8,92 & 534.135 & - & 9,14 & 534.135 & - & 9,01 & 534.135 \\
\hline 3 & 1.182 .322 & - & 219,58 & 1.182 .322 & - & 126,15 & 1.182 .322 & - & 939,00 & 1.182 .322 & - & $1.250,54$ & 1.182 .322 & - & $2.260,37$ & 1.182 .322 \\
\hline 4 & 3.117 .169 & 44,44 & 20,84 & 3.117 .169 & 44,44 & 14,02 & 3.692 .593 & 71,10 & 1,92 & 3.117 .169 & 44,44 & 12,69 & 2.158 .129 & - & 301,53 & 2.158 .129 \\
\hline 5 & 2.091 .636 & - & $1.426,30$ & 2.091 .636 & & $8.088,07$ & 2.091 .636 & . & $7.282,36$ & 2.091 .636 & - & $7.283,40$ & 2.091 .636 & - & $14.525,64$ & 2.091 .636 \\
\hline 6 & 34.478 .073 & 29,03 & $7.236,61$ & 34.478 .081 & 29,03 & $10.003,64$ & 35.389 .333 & 32,44 & $7.203,56$ & 30.956 .540 & 15,85 & $14.446,27$ & 26.720 .899 & - & $14.418,10$ & 26.720 .899 \\
\hline 7 & 22.209 .335 & - & $7.239,44$ & 22.209 .335 & - & $7.277,78$ & 24.558 .187 & 10,58 & 9,66 & 22.209 .335 & - & $7.274,46$ & 22.209 .335 & - & $7.260,73$ & 22.209 .335 \\
\hline 8 & 45.935 .944 & 22,78 & $7.224,37$ & 48.864 .400 & 30,61 & $14.453,76$ & 55.872 .952 & 49,34 & $7.226,18$ & 48.864 .400 & 30,61 & $14.477,03$ & 37.413 .879 & - & $14.443,50$ & 37.413 .879 \\
\hline 9 & 55.613 .386 & 21,16 & $7.439,57$ & 53.106 .600 & 15,70 & $14.510,69$ & 50.616 .467 & 10,28 & $7.206,28$ & 48.406 .461 & 5,46 & $12.595,52$ & 45.899 .670 & - & $14.418,48$ & 45.899 .670 \\
\hline 10 & 27.873 .155 & & $7.235,54$ & 27.873 .155 & & $2.582,22$ & 34.242 .341 & 22,85 & 21,10 & 27.873 .155 & & $2.032,79$ & 27.873 .155 & - & $7.248,34$ & 27.873 .155 \\
\hline 11 & 5.401 .753 & 0,28 & 187,33 & 5.401 .753 & 0,28 & $1.286,15$ & 5.401 .763 & 0,28 & 93,74 & 5.401 .755 & 0,28 & $7.364,19$ & 5.386 .504 & - & $17.399,89$ & 5.386 .504 \\
\hline 12 & 6.809 .135 & 9,75 & $8.925,18$ & 7.538 .927 & 21,51 & 40,61 & 6.725 .685 & 8,40 & 38,37 & 7.538 .927 & 21,51 & 81,56 & 6.204 .385 & - & 38,94 & 6.204 .385 \\
\hline 13 & 5.179 .632 & 64,18 & $7.256,73$ & 4.276 .074 & 35,54 & 9,67 & 3.154 .873 & - & $7.255,04$ & 4.703 .192 & 49,08 & $14.496,29$ & 3.573 .775 & 13,28 & $13.127,67$ & 3.154 .873 \\
\hline 14 & 4.644 .672 & 47,11 & 725,02 & 4.644 .672 & 47,11 & $1.188,85$ & 3.157 .296 & - & $7.703,51$ & 4.644 .642 & 47,11 & $15.945,36$ & 3.157 .296 & - & $16.371,01$ & 3.157 .296 \\
\hline 15 & 5.929 .694 & 0,00 & 6,35 & 5.929 .693 & 0,00 & 40,17 & 5.929 .689 & - & 322,76 & 5.929 .689 & - & 339,95 & 5.929 .689 & - & $3.251,44$ & 5.929 .689 \\
\hline 16 & 113.607 .775 & 18,55 & $21.653,22$ & 113.607 .758 & 18,55 & $28.307,68$ & 99.275 .793 & 3,59 & 566,12 & 95.833 .819 & - & $16.975,39$ & 95.833 .823 & 0,00 & $34.860,97$ & 95.833 .819 \\
\hline 17 & 123.637 .820 & 27,31 & $15.671,96$ & 103.579 .332 & 6,66 & $23.926,72$ & 115.161 .336 & 18,58 & $21.647,64$ & 98.921 .092 & 1,86 & $21.675,93$ & 97.113 .725 & . & $36.176,71$ & 97.113 .725 \\
\hline 18 & 83.691 .214 & 10,92 & $7.480,32$ & 75.451 .476 & - & $9.538,31$ & 75.451 .484 & 0,00 & $14.987,51$ & 86.930 .811 & 15,21 & $28.862,73$ & 78.691 .070 & 4,29 & $30.229,10$ & 75.451 .476 \\
\hline 19 & 76.464 .667 & 17,02 & $21.216,52$ & 71.631 .263 & 9,62 & $36.227,28$ & 65.345 .345 & 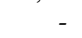 & $10.776,42$ & 79.607.694 & 21,83 & $23.981,38$ & 79.607 .694 & 21,83 & $32.478,90$ & 65.345 .345 \\
\hline 20 & 109.844 .482 & 0,61 & $7.514,74$ & 113.267 .334 & 3,75 & $23.674,16$ & 113.267 .308 & 3,75 & $14.421,08$ & 109.176 .594 & 0,00 & $14.505,50$ & 109.176 .573 & - & $28.970,24$ & 109.176 .573 \\
\hline 21 & 7.219 .381 & 22,40 & 695,71 & 6.695 .624 & 13,52 & $17.291,25$ & 7.005 .091 & 18,77 & $15.077,62$ & 7.005 .091 & 18,77 & $21.404,97$ & 5.898 .210 & - & $16.525,55$ & 5.898 .210 \\
\hline 22 & 8.207 .749 & 22,16 & $24.244,45$ & 7.253 .484 & 7,95 & $14.862,56$ & 9.015 .767 & 34,18 & $9.038,09$ & 6.719 .085 & - & $15.410,27$ & 6.719 .085 & - & $28.988,39$ & 6.719 .085 \\
\hline 23 & 7.974.488 & 18,56 & 77,33 & 7.115 .888 & 5,79 & $7.338,76$ & 6.726 .339 & - & $15.428,18$ & 7.362 .348 & 9,46 & $5.537,11$ & 7.871 .148 & 17,02 & $28.828,95$ & 6.726 .339 \\
\hline 24 & 8.202 .195 & 24,94 & $1.373,15$ & 8.202 .195 & 24,94 & $1.702,05$ & 6.564 .915 & - & $7.724,16$ & 8.202 .195 & 24,94 & $11.775,61$ & 8.202 .190 & 24,94 & $29.179,16$ & 6.564 .915 \\
\hline 25 & 6.664 .252 & 8,64 & $2.012,75$ & 6.895 .706 & 12,41 & $7.767,93$ & 6.134 .345 & - & $23.928,87$ & 6.743 .449 & 9,93 & $28.927,31$ & 6.298 .797 & 2,68 & $17.857,00$ & 6.134 .345 \\
\hline 26 & 272.344 .441 & 12,87 & $23.203,25$ & 269.652 .973 & 11,75 & $44.132,13$ & 253.706 .194 & 5,14 & $28.844,38$ & 241.294.077 & 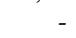 & $43.259,87$ & 245.270 .709 & 1,65 & $59.874,70$ & 241.294.077 \\
\hline 27 & 158.676 .666 & 17,80 & $22.168,96$ & 140.116 .932 & 4,02 & $43.249,50$ & 134.696 .175 & . & $28.824,49$ & 158.030 .460 & 17,32 & $36.144,31$ & 140.116 .850 & 4,02 & $72.167,00$ & 134.696 .175 \\
\hline 28 & 284.859 .603 & 4,10 & $28.940,70$ & 313.376 .844 & 14,53 & $59.314,40$ & 290.514 .704 & 6,17 & $28.856,63$ & 309.325 .850 & 13,05 & $43.306,10$ & 273.628.962 & - & $72.091,12$ & 273.628 .962 \\
\hline 29 & 215.762 .939 & 16,05 & $33.674,80$ & 238.705 .358 & 28,38 & $57.877,59$ & 185.930 .324 & - & $28.823,32$ & 222.306 .933 & 19,56 & $43.270,07$ & 208.526.087 & 12,15 & $72.153,97$ & 185.930 .324 \\
\hline 30 & 166.266 .161 & 19,10 & $37.376,09$ & 146.411 .526 & 4,88 & $62.693,13$ & 166.686 .099 & 19,40 & $28.848,61$ & 162.133 .831 & 16,14 & $43.330,91$ & 139.599 .601 & - & $72.158,99$ & 139.599 .601 \\
\hline \multicolumn{2}{|c|}{ Média (1-10) } & 12,67 & 4,52 & & 12,91 & 5. & & 19,66 & 0,09 & & 9,64 & 38 & & - & $7.488,70$ & \\
\hline \multirow{2}{*}{\multicolumn{2}{|c|}{$\begin{array}{l}\text { Média (11-20) } \\
\text { Média }(21-30)\end{array}$}} & 19,57 & $9.063,74$ & & 14,30 & $12.423,96$ & & 3,46 & $7.781,22$ & & 15,69 & $14.422,83$ & & 3,94 & $21.290,49$ & \\
\hline & & 16,66 & $17.376,72$ & & 12,82 & $31.622,93$ & & 8,37 & $21.539,44$ & & 12,92 & $29.236,65$ & & 6,25 & $46.982,48$ & \\
\hline \multicolumn{2}{|c|}{ Média (1-30) } & 16,30 & $10.081,66$ & & 13,34 & $16.584,31$ & & 10,50 & $10.770,25$ & & 12,75 & $16.532,62$ & & 3,40 & $25.253,89$ & \\
\hline
\end{tabular}


A Tabela 3.4 compara os resultados obtidos pela formulação exata completa, por uma versão do horizonte rolante, por uma do relax-and-fix e pela PDA. Como já dito, considerando a formulação exata completa $\left(\mathcal{M}_{p, P}^{2}\right)$ o CPLEX é capaz de resolver apenas instâncias pequenas quando $\xi=P+p-2$, por isso, consideramos apenas a resolução das instâncias de 1 a 10. Definimos o tempo limite de relógio do CPLEX para resolver o modelo $\mathcal{M}_{p, P}^{2}$ em 12 horas e para os outros três métodos definimos em 2 horas por subproblema resolvido.

Cada instância, com exceção das instâncias 28, 29 e 30, foi resolvida pela PDA em menos de 10 minutos (veja a Tabela 3.1), o que significa que o tempo limite de 10 minutos foi suficiente para encontrar a solução ótima de cada subproblema de cada instância. Analisando os logs gerados pelo CPLEX, apenas na instância 29 ao menos um subproblema não chegou à solução ótima em 10 minutos. Por isso, precisamos executar novamente apenas a instância 29 com o novo tempo limite de 2 horas por subproblema.

O CPLEX foi capaz de encontrar a solução ótima para quatro instâncias, nas outras seis a otimalidade não foi obtida no tempo limite, inclusive a solução obtida pelo $\mathcal{M}_{p, P}^{2}$, no tempo limite, na instância 9 foi maior do que a encontrada pelo $\operatorname{HR}(3-2)$ e $\operatorname{RF}(3-2)$. Nessa instância, a solução foi obtida pelo $\mathrm{HR}(3-2)$ e pelo $\mathrm{RF}(3-2)$ em um tempo bem inferior ao tempo limite do $\mathcal{M}_{p, P}^{2}$. Em média, o comportamento do $\mathrm{HR}(3-2)$ e $\mathrm{RF}(3-2)$ foi muito parecido. Em ambos, o tempo e o $R P D$ médio foram muito próximos. Entretanto, o $\mathrm{HR}(3-2)$ encontrou melhores soluções que o $\mathrm{RF}(3-2)$ nas instâncias 6, 15, 17, 19, 25 e 26, enquanto o $\mathrm{RF}(3-2)$ se saiu melhor nas instâncias 14, 16, 27, 28 e 30. Tanto o $\operatorname{HR}(3-2)$ quanto o $\operatorname{RF}(3-2)$ conseguiram obter a solução ótima para as instâncias 2 e 4. A PDA conseguiu encontrar 13 melhores soluções, sendo que os resultados encontrados para as instâncias 2 e 4 foram muito próximas da solução ótima e a solução obtida na instância 3 coincide com a solução obtida pelo CPLEX.

Na instância 1, apesar da PDA ter obtido uma solução rapidamente, tal solução tem um RPD muito alto, superior à $95 \%$. Como o $\operatorname{HR}(3-2)$ e o $\mathrm{RF}(3-2)$ também não conseguiram um solução de boa qualidade, isso indica que pelo menos um item do quarto período forçou a seleção de um novo objeto, fazendo com que a solução ficasse mais cara. Além disso, note que tanto o $\operatorname{HR}(3-2)$ quanto o $\mathrm{RF}(3-2)$ avançam um período por vez e, assim, na segunda iteração a janela de decisão já inclui os itens do quarto período. Como a solução foi muito pior que a solução ótima, podemos concluir que pelo menos um item do quarto período influenciou na decisão da seleção ou no tipo de corte de pelo menos um objeto do primeiro período.

Para as instâncias pequenas (1 a 10) os métodos heurísticos $\mathrm{HR}(3$ - 2) e o $\mathrm{RF}(3$ - 2) obtiveram um um RPD médio próximo de $18 \%$ e a PDA cerca de $21 \%$, mas o grande destaque ocorreu em relação o tempo gasto pela PDA, que foi muito inferior, chegando a ser mais de 1000 vezes mais rápida do que o $\mathcal{M}_{p, P}^{2}$ e mais de 250 vezes mais rápida que o $\mathrm{HR}(3-2)$ e o $\mathrm{RF}(3-2)$. Para as instâncias 11 a 20 a PDA conseguiu, na grande maioria, soluções bem melhores que as obtidas pelo $\operatorname{HR}(3-2)$ ou $\mathrm{RF}(3-2)$ e consumindo um tempo muito inferior. Nas instâncias 21 a 30, os três métodos tiveram um RPD médio relativamente próximo, mas, novamente, a PDA fui muito mais eficiente, sendo mais de 50 vezes mais rápida, em média, que os outros dois métodos. 
Tabela 3.4: Resultado numérico para as instâncias do PCBMPSA considerando diferentes métodos

\begin{tabular}{|c|c|c|c|c|c|c|c|c|c|c|c|c|c|}
\hline \multirow[t]{2}{*}{ Inst. } & \multicolumn{3}{|c|}{$\mathcal{M}_{p, P}^{2}$} & \multicolumn{3}{|c|}{$\mathrm{HR}(3-2)$} & \multicolumn{3}{|c|}{$\mathrm{RF}(3-2)$} & \multicolumn{3}{|c|}{ PDA } & \multirow{2}{*}{$\begin{array}{l}\text { Melhor } \\
\text { Solução }\end{array}$} \\
\hline & Solução & RPD & Tempo & Solução & RPD & Tempo & Solução & RPD & Tempo & Solução & RPD & Tempo & \\
\hline 1 & 371.057 & - & $29.372,46$ & 664.729 & 79,14 & 4,21 & 664.729 & 79,14 & 1,31 & 726.563 & 95,81 & 5,73 & 371.057 \\
\hline 2 & 534.135 & - & $1.146,87$ & 534.135 & - & 9,77 & 534.135 & - & 9,01 & 534.198 & 0,01 & 6,97 & 534.135 \\
\hline 3 & 1.182 .322 & - & $43.205,04$ & 1.182 .322 & - & $7.939,06$ & 1.182 .322 & - & $2.260,37$ & 1.182 .322 & - & 9,46 & 1.182 .322 \\
\hline 4 & 2.158 .129 & - & 236,89 & 2.158 .129 & - & 330,89 & 2.158 .129 & - & 301,53 & 2.917 .570 & 35,19 & 7,99 & 2.158 .129 \\
\hline 5 & 2.091 .636 & - & $43.202,48$ & 2.091 .636 & - & $14.505,18$ & 2.091 .636 & - & $14.525,64$ & 2.091 .662 & 0,00 & 15,86 & 2.091 .636 \\
\hline 6 & 23.888 .977 & - & $43.204,96$ & 26.720 .887 & 11,85 & $7.364,47$ & 26.720 .899 & 11,85 & $14.418,10$ & 26.721 .158 & 11,86 & 70,90 & 23.888.977 \\
\hline 7 & 13.715 .629 & - & $3.206,22$ & 22.209 .335 & 61,93 & $7.272,18$ & 22.209 .335 & 61,93 & $7.260,73$ & 16.899 .377 & 23,21 & 13,44 & 13.715 .629 \\
\hline 8 & 37.413 .879 & - & $43.202,99$ & 37.413 .879 & - & $14.450,68$ & 37.413 .879 & - & $14.443,50$ & 45.935 .984 & 22,78 & 46,17 & 37.413.879 \\
\hline 9 & 48.406 .490 & 5,46 & $43.200,17$ & 45.899 .670 & - & $14.422,81$ & 45.899 .670 & - & $14.418,48$ & 55.614 .336 & 21,17 & 78,11 & 45.899 .670 \\
\hline 10 & 21.474 .855 & - & $43.202,52$ & 27.873 .155 & 29,79 & $7.243,35$ & 27.873.155 & 29,79 & $7.248,34$ & 21.474 .954 & 0,00 & 31,79 & 21.474 .855 \\
\hline 11 & & & & 5.386 .504 & 22,12 & $18.607,18$ & 5.386 .504 & 22,12 & $17.399,89$ & 4.411 .006 & - & 25,60 & 4.411 .006 \\
\hline 12 & & & & 6.204 .385 & 2,06 & 32,64 & 6.204 .385 & 2,06 & 38,94 & 6.079 .436 & - & 21,14 & 6.079 .436 \\
\hline 13 & & & & 3.573 .767 & 0,46 & $17.699,57$ & 3.573 .775 & 0,46 & $13.127,67$ & 3.557 .438 & - & 17,66 & 3.557 .438 \\
\hline 14 & & & & 4.644 .672 & 47,11 & $22.749,00$ & 3.157 .296 & - & $16.371,01$ & 3.754 .328 & 18,91 & 18,56 & 3.157 .296 \\
\hline 15 & & & & 4.400 .599 & - & 566,08 & 5.929 .689 & 34,75 & $3.251,44$ & 4.400 .755 & 0,00 & 9,72 & 4.400 .599 \\
\hline 16 & & & & 95.833 .848 & 0,00 & $28.927,78$ & 95.833 .823 & - & $34.860,97$ & 98.864 .853 & 3,16 & 76,06 & 95.833 .823 \\
\hline 17 & & & & 96.502 .913 & 3,24 & $23.370,25$ & 97.113 .725 & 3,89 & $36.176,71$ & 93.474 .193 & - & 204,96 & 93.474.193 \\
\hline 18 & & & & 78.691 .081 & 4,29 & $28.675,68$ & 78.691 .070 & 4,29 & $30.229,10$ & 75.451 .522 & - & 95,74 & 75.451 .522 \\
\hline 19 & & & & 58.161 .163 & - & $27.480,34$ & 79.607 .694 & 36,87 & $32.478,90$ & 60.195 .048 & 3,50 & 68,69 & 58.161 .163 \\
\hline 20 & & & & 109.176 .588 & 2,16 & $29.095,91$ & 109.176.573 & 2,16 & $28.970,24$ & 106.867 .140 & - & 94,45 & 106.867 .140 \\
\hline 21 & & & & 5.898 .210 & 8,42 & $16.114,89$ & 5.898 .210 & 8,42 & $16.525,55$ & 5.440 .012 & - & 52,16 & 5.440 .012 \\
\hline 22 & & & & 6.719 .085 & - & $28.977,93$ & 6.719 .085 & - & $28.988,39$ & 7.202 .654 & 7,20 & 135,70 & 6.719 .085 \\
\hline 23 & & & & 7.871 .148 & 17,85 & $31.485,15$ & 7.871.148 & 17,85 & $28.828,95$ & 6.678 .827 & - & 26,94 & 6.678 .827 \\
\hline 24 & & & & 8.202 .190 & 4,54 & $29.090,40$ & 8.202 .190 & 4,54 & $29.179,16$ & 7.845 .955 & - & 43,56 & 7.845 .955 \\
\hline 25 & & & & 6.134 .329 & - & $46.113,14$ & 6.298 .797 & 2,68 & $17.857,00$ & 7.182 .102 & 17,08 & 43,93 & 6.134 .329 \\
\hline 26 & & & & 234.867 .219 & - & $58.124,94$ & 245.270 .709 & 4,43 & $59.874,70$ & 256.237 .067 & 9,10 & 429,70 & 234.867.219 \\
\hline 27 & & & & 142.091 .383 & 1,41 & $68.767,96$ & 140.116 .850 & - & $72.167,00$ & 147.836 .095 & 5,51 & 321,24 & 140.116 .850 \\
\hline 28 & & & & 280.407 .250 & 4,75 & $72.186,72$ & 273.628 .962 & 2,22 & $72.091,12$ & 267.693 .154 & - & 846,69 & 267.693 .154 \\
\hline 29 & & & & 208.526 .094 & 8,86 & $60.416,20$ & 208.526 .087 & 8,86 & $72.153,97$ & 191.550 .586 & - & $5.993,35$ & 191.550 .586 \\
\hline 30 & & & & 144.958 .772 & 6,30 & $71.630,59$ & 139.599 .601 & 2,37 & $72.158,99$ & 136.371 .356 & - & 807,95 & 136.371 .356 \\
\hline \multicolumn{2}{|c|}{ Média (1-10) } & 0,55 & $29.318,06$ & & 18,27 & $7.354,26$ & & 18,27 & $7.488,70$ & & 21,00 & 28,64 & \\
\hline \multirow{2}{*}{\multicolumn{2}{|c|}{$\begin{array}{l}\text { Média (11-20) } \\
\text { Média (21-30) }\end{array}$}} & & & & 8,14 & $19.720,44$ & & 10,66 & $21.290,49$ & & 2,56 & 63,26 & \\
\hline & & & & & 5,21 & $48.290,79$ & & 5,14 & $46.982,48$ & & 3,89 & 870,12 & \\
\hline \multicolumn{2}{|c|}{ Média (1-30) } & & & & 10,54 & $25.121,83$ & & 11,36 & $25.253,89$ & & 9,15 & 320,67 & \\
\hline
\end{tabular}

†: Tempo limite de $12 \mathrm{~h}$ atingido 


\section{Capítulo 4}

\section{Planejamento sob incertezas}

Nos capítulos anteriores, apresentamos um modelo e algumas técnicas baseadas em heurísticas para resolver uma versão determinística do PCBMPSA. Por determinística, queremos dizer que os itens das ordens de trabalho e o custo por unidade de área de cada objeto disponível no horizonte de planejamento são conhecidos à priori. Na prática, entretanto, a suposição de que todos os dados do modelo são conhecidos à priori, limita sua utilidade no planejamento sob incertezas [49]. Nesse caso, nem todas as informações estão disponíveis no início do planejamento e alguns parâmetros podem ser representados por variáveis aleatórias com alguma distribuição de probabilidade conhecida.

Para o problema em questão, consideramos que a distribuição de probabilidade dos parâmetros incertos (variáveis aleatórias), isto é, a distribuição de probabilidade da quantidade de cada tipo de item e a distribuição de probabilidade do custo de cada objeto disponível, são conhecidas ou podem ser razoavelmente bem estimadas. Isso faz sentido em situações nas quais tais distribuições de probabilidade podem ser estimadas a partir dos dados históricos.

Como não temos todos os dados de entrada de antemão, não podemos resolvê-lo como feito nos capítulos anteriores. Entretanto, usando as distribuições de probabilidade, podemos gerar amostras de dados e usá-las na PDA visando calcular as estimativas da proporção de utilização das sobras de cada objeto, ou seja, usamos a PDA para resolver o problema no cenário sob incerteza. Nesse caso, a seguinte estratégia pode ser adotada: para cada iteração da PDA, geramos uma amostra dos dados de cada período, seguindo a distribuição de probabilidade da quantidade de cada tipo de item e a distribuição de probabilidade do custo de cada objeto, e resolvemos a PDA, como feito anteriormente. Ao fim, quando a PDA atingir o critério de parada, teremos as estimativas da proporção de utilização das sobras de cada objeto ajustadas seguindo as distribuições de probabilidade. Dessa forma, a PDA tenta aprender o que pode acontecer ao longo do horizonte de planejamento, com o objetivo de economizar no futuro, podendo indicar a compra antecipada de algum objeto maior (possivelmente mais caro), em um determinado período.

Perceba que no planejamento sob incerteza não podemos utilizar o horizonte rolante (com $\Delta \geq 2$ ) e o relax-and-fix, uma vez que os mesmos precisam conhecer os dados de antemão para serem resolvidos. Por outro lado, podemos usar o método guloso (que é equivalente ao horizonte rolante $\operatorname{com} \Delta=1$ ) para resolver o problema considerando apenas os dados do período atual. Entretanto, 
como o método guloso não conhece/usa as informações futuras (os itens solicitados nas ordens de trabalho e o custo dos objetos), ele pode encontrar uma solução global cuja soma do custo dos objetos utilizados seja muito maior do que realmente é necessário. Em outras palavras, como já dito, o método guloso pode economizar em determinado período, mas em outro o gasto pode ser muito elevado, devido a não utilização das informações futuras.

Em cenários reais, é comum encontrar promoções de matéria-prima (objetos), em alguns períodos. Nesse caso, seria uma boa alternativa adquirir o máximo possível desses objetos de custo reduzido. Entretanto, essa compra antecipada não acontece na prática, devido, entre outros fatores, à restrição de fluxo de caixa da empresa. Com isso, supomos que é permitido gastar $\rho \in \mathbb{R}$ vezes o custo mínimo necessário para atender a ordem de trabalho de cada período. Por exemplo, se no primeiro período precisamos comprar objetos, para atender a ordem de trabalho dos itens, cujo custo é de 100 unidades monetárias e considerando que a empresa está disposta a gastar, no máximo, o dobro $(\rho=2)$ desse valor na antecipação da compra de objetos ou na compra de objetos mais caros, visando uma economia futura, teremos uma restrição adicional que limita a compra de objetos ao custo de, no máximo, 200 unidades monetárias. Assim, temos uma restrição adicional no modelo usado pela PDA, que é dada por

$$
\sum_{j=1}^{m_{s}} c_{s j} W_{s j} H_{s j} u_{s j} \leq C_{s}^{\max }, \quad s=p, \ldots, P,
$$

onde, $C_{s}^{\max }$ é o custo máximo que a empresa pode gastar no período $s$ com a compra de objetos.

O valor de $C_{s}^{\max }$ é definido da seguinte forma: encontramos o custo mínimo $C_{s}^{\min }$ dos objetos necessários para cortar os itens da ordem de servido do período $s$ resolvendo o modelo $\mathcal{C}$ (apresentado na Seção 2.1) e multiplicamos esse custo $C_{s}^{\min }$ por $\rho$, ou seja, $C_{s}^{\max }=\rho C_{s}^{\min }$. Assim, em cada iteração da PDA, após a amostra dos dados ser gerada, resolvemos o modelo $\mathcal{C}$, para encontrar o valor $C_{s}^{\max }$ de cada período, e adicionamos a restrição (4.1) ao modelo $\tilde{\mathcal{M}}_{s, s}$. Chamaremos o $\tilde{\mathcal{M}}_{s, s}$ com a restrição adicional (4.1) de $\tilde{\mathcal{M}}_{s, s}^{\mathrm{c}_{s}^{\max }}$.

Note que não precisamos adicionar restrição (4.1) ao modelo resolvido pelo método guloso, uma vez que o mesmo nunca antecipa ou compra objetos mais caros do que o necessário em cada período.

\subsection{PDA não-determinística}

No planejamento sob incertezas, consideramos um cenário em que conhecemos de antemão os $\tilde{n}$ tipos de itens que podem ser solicitados nas ordens de trabalho e os $\tilde{m}$ tipos de objetos que podem ser usados, mas não sabemos a quantidade de cada tipo de item nas ordens de trabalho e nem o custo por unidade de área de cada tipo de objeto em cada período. Nesse cenário, supomos que temos apenas a média e o desvio padrão dessas informações que seguem uma distribuição normal. Assim, em cada iteração da PDA, podemos gerar amostras aleatórias com essas informações e usá-las para estimar a proporção de utilização futura das sobras dos objetos. Para que seja viável usar a PDA, é esperado que as amostras geradas sejam similares.

Para estimar a quantidade $q_{s i}^{\mathrm{I}}$ de cada tipo de item $i$ no período $s$, sorteamos essa quantidade, 
usando um gerador de números aleatórios com semente seed, seguindo a distribuição normal, com média $\mu_{s i}^{\mathrm{I}}$ e desvio padrão $\sigma_{s i}^{\mathrm{I}}$, associada à quantidade do tipo de item de cada período. Além disso, essa quantidade deve ser inteira e maior que zero. Assim, definindo $\tilde{\mathcal{N}}^{\mathrm{I}}\left(\right.$ seed, $\left.\mu_{s i}^{\mathrm{I}}, \sigma_{s i}^{\mathrm{I}}\right)=$ $\max \left\{\left\lfloor\mathcal{N}\left(\mu_{s i}^{\mathrm{I}}, \sigma_{s i}^{\mathrm{I}}\right)+0,5\right\rfloor, 0\right\}$, temos que $q_{s i}^{\mathrm{I}}=\tilde{\mathcal{N}}^{\mathrm{I}}\left(\right.$ seed, $\left.\mu_{s i}^{\mathrm{I}}, \sigma_{s i}^{\mathrm{I}}\right)$, para $s=p, \ldots, P, i=1, \ldots, \tilde{n}$. De forma similar, o custo por unidade de área $\tilde{c}_{s j}$, do objeto de tipo $j$ no período $s$, é estimado usando um gerador de números aleatórios com semente seed e seguindo a distribuição normal, de média $\mu_{s j}^{\mathrm{O}}$ e desvio padrão $\sigma_{s j}^{\mathrm{O}}$, relacionada ao custo do objeto. Por se tratar de um custo, não faz sentido $\tilde{c}_{s j}$ ser menor ou igual a zero, por isso $\tilde{c}_{s j}$ deve ser maior que 0,01 (um centavo) por unidade de área. Desse modo, definindo $\tilde{\mathcal{N}}^{\mathrm{C}}\left(\right.$ seed $\left., \mu_{s j}^{\mathrm{O}}, \sigma_{s j}^{\mathrm{O}}\right)=\max \left\{\mathcal{N}\left(\mu_{s j}^{\mathrm{O}}, \sigma_{s j}^{\mathrm{o}}\right), 0,01\right\}$, temos que $\tilde{c}_{s j}=\tilde{\mathcal{N}}^{\mathrm{C}}\left(s e e d, \mu_{s j}^{\mathrm{O}}, \sigma_{s j}^{\mathrm{O}}\right)$, para $s=p, \ldots, P, j=1, \ldots, \tilde{m}$.

Inicialmente consideramos que a disponibilidade de cada tipo de objeto em cada período é ilimitada, mas é fácil notar que o número de objetos de cada tipo em cada período pode ser limitado pelo maior número de itens que cada período pode conter. Usando a distribuição de probabilidade normal de média $\mu_{s i}^{\mathrm{I}}$ e desvio padrão $\sigma_{s i}^{\mathrm{I}}$, a maior quantidade do item do tipo $i$, que pode ser gerada com $99,7 \%$ de probabilidade no período $s$, é dada por $\widetilde{q}_{s i}^{\mathrm{I}}=\left\lceil\mu_{s i}^{\mathrm{I}}+3 \times \sigma_{s i}^{\mathrm{I}}\right\rceil$. Assim, um limitante superior para a quantidade disponível do objeto de tipo $j$ no período $s$ pode ser dada por $\sum_{i=1}^{\tilde{n}} \widetilde{q}_{s i}^{\mathrm{I}}$, ou seja, um objeto do tipo $j$ para cada item. Entretanto, esse valor pode ser muito grande e, com o objetivo de melhorar esse limitante e assim diminuir a complexidade de outros modelos que precisem do número de objetos de cada tipo, podemos estimar um limitante superior mais justo para o número de objetos do tipo $j$ no período $s$ necessários para empacotar simultaneamente os $\sum_{i=1}^{\tilde{n}} \tilde{q}_{s i}^{\mathrm{I}}$ itens.

Um limitante superior para o número de objetos de um determinado tipo, necessário para empacotar uma lista de itens, pode ser obtido resolvendo um modelo exato para o problema de empacotamento bidimensional (2DBPP) [12,38]. Nesse problema, dado um estoque ilimitado de objetos com comprimento $W$ e altura $H$ e uma coleção $I=1, \ldots, n$ de itens, tal que cada item $i$ possui largura $w_{i} \leq W$ e altura $h_{i} \leq H$, o objetivo é empacotar todos os itens utilizando o menor número de objetos. Os itens não podem se sobrepor e rotações não são consideradas. Além disso, $W$ e $H$ bem como $w_{i}$ e $h_{i}$ (para todo $i \in I$ ) são inteiros positivos maiores que zero. O modelo MIP para o problema consiste nas seguintes variáveis: a variável inteira $m_{i}$ indica o número do objeto que contém o item $i$; as variáveis $x_{i}$ e $y_{i}$ indicam a coordenada inferior esquerda do posicionamento do item $i$ no objeto $m_{i}$; a variável binária $l_{i j}$ vale 1 se e somente se o item $i$ estiver localizado à esquerda do item $j$; a variável binária $b_{i j}$ vale 1 se e somente se o item $i$ estiver abaixo do item $j$; a variável binária $p_{i j}$ vale 1 se e somente se $m_{i}<m_{j}$; e, finalmente, a variável $v$ indica o número de objetos usados para empacotar todos os itens.

Para garantir que dois itens não se sobreponham, as seguintes inequações devem ser satisfeitas:

$$
l_{i j}+l_{j i}+b_{i j}+b_{j i}+p_{i j}+p_{j i} \geq 1 \quad i, j \in I, i<j .
$$

Se o item $i$ estiver posicionado à esquerda de $j\left(l_{i j}=1\right)$, então temos que $x_{i}+w_{i} \leq x_{j}$. De forma similar, se o item $i$ estiver posicionado abaixo do item $j\left(b_{i j}=1\right)$, então $y_{i}+h_{i} \leq y_{j}$. Nenhuma parte do item deve exceder o objeto, assim $0 \leq x_{i} \leq W-w_{i}$ e $0 \leq y_{i} \leq H-h_{i}$. Além disso, como $v$ é o 
número de objetos utilizados, temos que $1 \leq m_{i} \leq v$. Para reduzir o número de soluções simétricas, acrescentamos uma restrição adicional $m_{i} \leq i$ ao modelo, exigindo que o item 1 seja empacotado no primeiro objeto, o item 2 seja empacotado em um dos dois primeiros objetos e assim por diante. Usando essas variáveis e restrições, o modelo do problema, chamado de $\mathcal{L}^{\mathrm{O}}$, pode ser formulado como o seguinte MIP:

$$
\begin{aligned}
& \text { Minimizar } v \\
& \text { s.a } l_{i j}+l_{j i}+b_{i j}+b_{j i}+p_{i j}+p_{j i} \geq 1 \quad i, j \in I, i<j, \\
& x_{i}-x_{j}+W l_{i j} \leq W-w_{i} \quad i, j \in I, \\
& y_{i}-y_{j}+H b_{i j} \leq H-h_{i} \quad i, j \in I \text {, } \\
& m_{i}-m_{j}+n p_{i j} \leq n-1 \quad i, j \in I \text {, } \\
& 0 \leq x_{i} \leq W-w_{i} \quad i \in I, \\
& 0 \leq y_{i} \leq H-h_{i} \quad i \in I \text {, } \\
& 1 \leq m_{i} \leq v \quad i \in I, \\
& m_{i} \leq i \quad i \in I, \\
& l_{i j}, b_{i j}, p_{i j} \in\{0,1\} \quad i, j \in I, \\
& x_{i}, y_{i} \in \mathbb{R} \quad i \in I \text {, } \\
& m_{i}, v \in \mathbb{N} \quad i \in I .
\end{aligned}
$$

Após descrevermos, para cada período, como as amostras são geradas e como definimos um limitante superior para o número de objetos de cada tipo, podemos apresentar o algoritmo responsável pelo ajuste dos parâmetros da PDA, isto é, podemos determinar as estimativas da proporção de utilização das sobras de cada objeto, baseado nas amostras geradas em cada iteração. O Algoritmo 4.1 é responsável por esse ajuste, que recebe vinte parâmetros, a saber:

- seed: semente para a geração de números aleatórios;

- $p$ e $P$ : período inicial e final, respectivamente, do horizonte de planejamento;

- $\tilde{n}$ : número de tipos de itens;

- $\tilde{w}_{i}$ e $\tilde{h}_{i}$ : largura e altura, respectivamente, do item de tipo $i=1, \ldots, \tilde{n}$ que pode ser solicitado em uma ordem de trabalho;

- $\mu_{s i}^{\mathrm{I}}$ e $\sigma_{s i}^{\mathrm{I}}$ : média e desvio padrão, respectivamente, da quantidade do item de tipo $i=1, \ldots, \tilde{n}$ na ordem de trabalho do período $s=p, \ldots, P$;

- $d$ : quantidade de itens do catálogo;

- $\bar{w}_{i}$ e $\bar{h}_{i}$ : comprimento e altura, respectivamente, do item $i=1, \ldots, d$ do catálogo;

- $\tilde{m}$ : número de tipos de objetos;

- $\tilde{W}_{j}$ e $\tilde{H}_{j}$ : largura e altura, respectivamente, do objeto de tipo $j=1, \ldots, \tilde{m}$ que pode ser utilizado; 
- $\mu_{s j}^{\mathrm{o}}$ e $\sigma_{s j}^{\mathrm{o}}$ : média e desvio padrão, respectivamente, do custo por unidade de área do objeto de tipo $j=1, \ldots, \tilde{m}$ no período $s=p, \ldots, P$;

- $\delta_{\text {ini: }}$ fração inicial das estimativas de proporção de utilização das sobras dos objetos;

- $\sigma$ : tamanho do passo usado em (3.6);

- $\epsilon$ : parâmetro de tolerância de erro usado em (3.7);

- $\rho$ : fator multiplicativo do custo que a empresa pode gastar, além do mínimo necessário, na compra de objetos.

Inicialmente o Algoritmo 4.1 determina, para cada período, um limitante superior para a quantidade $q_{s j}^{\mathrm{O}}$ de cada objeto de tipo $j$, obtido resolvendo o modelo $\mathcal{L}^{\mathrm{O}}$. Em seguida, são gerados $q_{s j}^{\mathrm{O}}$ réplicas do objeto de tipo $j$, que possui largura $\tilde{W}_{j}$ e altura $\tilde{H}_{j}$, e o número de objetos disponíveis $m_{s}$, em cada período, é atualizado. Feito isso, definimos a quantidade $\bar{m}_{s}$ de objetos que podem estar disponíveis em cada período $s$ (número de objetos de entrada do período $s$ mais as duas possíveis sobras aproveitáveis de cada objeto de entrada dos períodos anteriores) e as estimativas de proporção de utilização das sobras futuras dos $\bar{m}_{s}$ objetos de cada período $s=p, \ldots, P$ são inicializadas. Em seguida, o algoritmo inicia a fase de calibração dos parâmetros, que é executada até que o critério de parada (3.7) seja satisfeito. Como dissemos anteriormente, a quantidade de cada tipo de item e o custo por unidade de área de cada tipo de objeto seguem uma distribuição de probabilidade e, em cada iteração $k$, esses valores são gerados para cada período. Entre as linhas 14 e 18, definimos a quantidade $q_{s i}^{\mathrm{I}}$ do item de tipo $i$ na ordem de trabalho do período $s$ e, em seguida, atribuímos a largura $w_{i}$ e a altura $h_{i}$ de todos os itens da ordem de trabalho, que são compostos por $q_{s i}^{\mathrm{I}}$ cópias do item de tipo $i=1, \ldots, \tilde{n}$, e também calculamos a quantidade total $n_{s}$ de itens no período $s$. Entre as linhas 20 e 24, estipulamos o custo por unidade de área $\tilde{c}_{s j}$ do objeto de tipo $j$ no período $s$ e replicamos esse valor para todos os $q_{s j}^{\mathrm{O}}$ objetos do mesmo tipo.

Com os dados do período $s$ definidos, calculamos o custo $C_{s}^{\max }$, resolvendo o modelo $\mathcal{C}$, e, em seguida, resolvemos o modelo proposto para o problema de empacotamento com sobras aproveitáveis $\left(\tilde{\mathcal{M}}_{s, s}^{c_{s}^{\text {max }}}\right)$. Utilizando os valores das variáveis encontradas na resolução do modelo $\tilde{\mathcal{M}}_{s, s}^{\mathrm{C}_{s}^{\text {max }}}$, calculamos a largura da sobra da direita $\left(r_{s j}\right)$ e a altura da sobra superior $\left(t_{s j}\right)$ de cada objeto do período $s$. Além disso, caso $s<P$, determinamos as dimensões e o valor por unidade de área da sobra da direita e da sobra superior de cada um dos $\bar{m}_{s}$ objetos do período $s$. Como tais sobras devem estar disponíveis no próximo período para serem utilizadas, na linha 32, atribuímos as dimensões e o custo por unidade de área de cada sobra em sua posição correta no período $s+1$. Perceba que no período $s+1$, os primeiros $m_{s+1}$ objetos são os objetos de entrada (gerados no início do treinamento) e as próximas posições $\left(m_{s+1}+1\right.$ até $\left.\bar{m}_{s+1}\right)$ são objetos originados de sobras dos períodos anteriores.

Após a resolução do problema para o horizonte de planejamento na iteração $k$, o próximo passo é calcular a estimativa da proporção de utilização futura de cada sobra, dada por (3.6). Para isso, precisamos saber a área utilizada pela sobra da direita e pela sobra superior dos objetos de cada período. Assim, sejam $A_{s j}^{\mathrm{r}}$ e $A_{s j}^{\mathrm{t}}$ a soma das áreas dos itens cortados, respectivamente, na sobra da direita e na sobra superior no objeto $j$ do período $s$; e seja $A_{s j}$ a área total utilizada no objeto $j$ no período $s$, ou seja, $A_{s j}=\sum_{i=1}^{n_{s}} v_{s i j} w_{s i} h_{s i}+A_{s j}^{\mathrm{r}}+A_{s j}^{\mathrm{t}}$. Inicialmente, na linha 36, determinamos a soma 
das áreas dos itens que foram atribuídos a cada objeto originado de uma sobra. Note que no último período só podemos ter itens cortados na área de corte do objeto $j \mathrm{e}$, assim, as sobras desse objeto não são utilizadas para cortar itens das ordens de trabalho. Por outro lado, nos períodos $s<P$, as sobras de cada objeto podem ter sido utilizadas para cortar itens de ordens de trabalho futuras. Como as sobras da direita dos objetos do período $s$ são posicionadas uma após a outra, em sequência, após os $m_{s+1}$ objetos do período $s+1$ e as sobras superiores são posicionadas uma após a outra após as sobras da direita, no período $s<P$, a área utilizada pela sobra da direita do objeto $j$ corresponde a área total utilizada pelo objeto $m_{s+1}+j$ do período $s+1$ e a sobra superior corresponde ao objeto $m_{s+1}+\bar{m}_{s}+j$ do período $s+1$. Dessa forma, quando $s<P, A_{s j}^{\mathrm{r}}=A_{s+1, m_{s+1}+j}$ e $A_{s j}^{\mathrm{t}}=A_{s+1, m_{s+1}+\bar{m}_{s}+j}$. Definida a área utilizada por cada sobra, podemos determinar as novas estimativas da proporção de utilização das sobras dos $\bar{m}_{s}$ objetos, baseadas na amostra gerada pela iteração $k$. Na linha 52 atualizamos a área total utilizada pelo objeto, que corresponde a área dos itens cortados na área de corte do objeto somado com a área dos itens cortados na sobra da direita e na sobra superior do objeto.

Quando o critério de parada (3.7) for satisfeito o algoritmo retorna, para cada período $s=p, \ldots, P$, os valores das estimativas da proporção de utilização futura da sobra da direita e da sobra superior, encontrada na última iteração, de cada objeto $j=1, \ldots, \bar{m}_{s}$.

\section{Algoritmo 4.1 Treinamento}

Entrada: $\operatorname{seed}, p, P, \tilde{n}, \widetilde{w}_{i}, \tilde{h}_{i}(i=1, \ldots, \tilde{n}), \mu_{s i}^{\mathrm{I}}, \sigma_{s i}^{\mathrm{I}}(s=p, \ldots, P$ e $i=1, \ldots, \tilde{n}), d, \bar{w}_{i}, \bar{h}_{i}(i=1, \ldots, d)$, $\tilde{m}, \tilde{W}_{j}, \tilde{H}_{j}(j=1, \ldots, \tilde{m}), \mu_{s j}^{\mathrm{O}}, \sigma_{s j}^{\mathrm{O}}(s=p, \ldots, P$ e $j=1, \ldots, \tilde{m}), \delta_{\text {ini }}, \sigma, \epsilon, \rho$.

Saída: $\delta_{s, j, k+1}^{\mathrm{r}}, \delta_{s, j, k+1}^{\mathrm{t}}\left(s=p, \ldots, P, j=1, \ldots, \bar{m}_{s}\right)$.

Treinamento $\left(s e e d, p, P, \tilde{n}, \tilde{w}, \tilde{h}, \mu^{\mathrm{I}}, \sigma^{\mathrm{I}}, d, \bar{w}, \bar{h}, \tilde{m}, \tilde{W}, \tilde{H}, \mu^{\mathrm{O}}, \sigma^{\mathrm{O}}, \delta_{\text {ini }}, \sigma, \epsilon, \rho\right)$.

1: for $s \leftarrow p$ to $P$ do $\quad \triangleright$ Calcula a quantidade disponível para cada tipo de objeto em cada período

2: $\quad m_{s} \leftarrow 0$

3: $\quad$ for $j \leftarrow 1$ to $\tilde{m}$ do

4: $\quad$ Atribua a $q_{s j}^{\mathrm{O}}$ um limitante superior para a quantidade de objetos de largura $\tilde{W}_{j}$ e altura $\tilde{H}_{j}$ que são necessários para empacotar simultaneamente $\left\lceil\mu_{s i}^{\mathrm{I}}+3 \times \sigma_{s i}^{\mathrm{I}}\right\rceil$ itens com dimensões $\tilde{w}_{i}$ e $\tilde{h}_{i}$ para $i \in\left\{1, \ldots, \tilde{n} \mid \tilde{w}_{i} \leq \tilde{W}_{j}\right.$ e $\left.\tilde{h}_{i} \leq \tilde{H}_{j}\right\}$ resolvendo o modelo $\mathcal{L}^{\mathrm{O}}$.

5: $\quad$ Defina $W_{s, m_{s}+\ell}=\tilde{W}_{j}$ e $H_{s, m_{s}+\ell}=\tilde{H}_{j}$, para $\ell=1, \ldots, q_{s j}^{\mathrm{O}}$.

6:

7: end for $m_{s} \leftarrow m_{s}+q_{s j}^{\mathrm{O}}$

8: end for

9: Defina $\bar{m}_{s}=m_{s}+2\left(\sum_{i=1}^{\min (s-p, P+p-2)} 2^{i-1} m_{s-i}\right)$, para $s=p, \ldots, P$.

10: Defina $\delta_{s j 1}^{\mathrm{r}}=\delta_{s j 1}^{\mathrm{t}}=\delta_{\text {ini }}$, para $s=p, \ldots, P-1, j=1, \ldots, \bar{m}_{s}$, e $\delta_{P j 1}^{\mathrm{r}}=\delta_{P j 1}^{\mathrm{t}}=0$, para $j=1, \ldots, \bar{m}_{P}$.

11: for $k \leftarrow 1$ until o critério de parada (3.7) ser satisfeito do

for $s \leftarrow p$ to $P$ do

$n_{s} \leftarrow 0$

for $i \leftarrow 1$ to $\tilde{n}$ do

$q_{s i}^{\mathrm{I}} \leftarrow \widehat{\mathcal{N}}^{\mathrm{I}}\left(\right.$ seed $\left., \mu_{s i}^{\mathrm{I}}, \sigma_{s i}^{\mathrm{I}}\right) \quad \triangleright$ Quantidade do item de tipo $i$ na ordem de trabalho do período $s$ $w_{s, n_{s}+\ell} \leftarrow \tilde{w}_{i}$ e $h_{s, n_{s}+\ell} \leftarrow \tilde{h}_{i}$, para $\ell=1, \ldots, q_{s i}^{\mathrm{I}}$.

$n_{s} \leftarrow n_{s}+q_{s i}^{\mathrm{I}}$

end for 


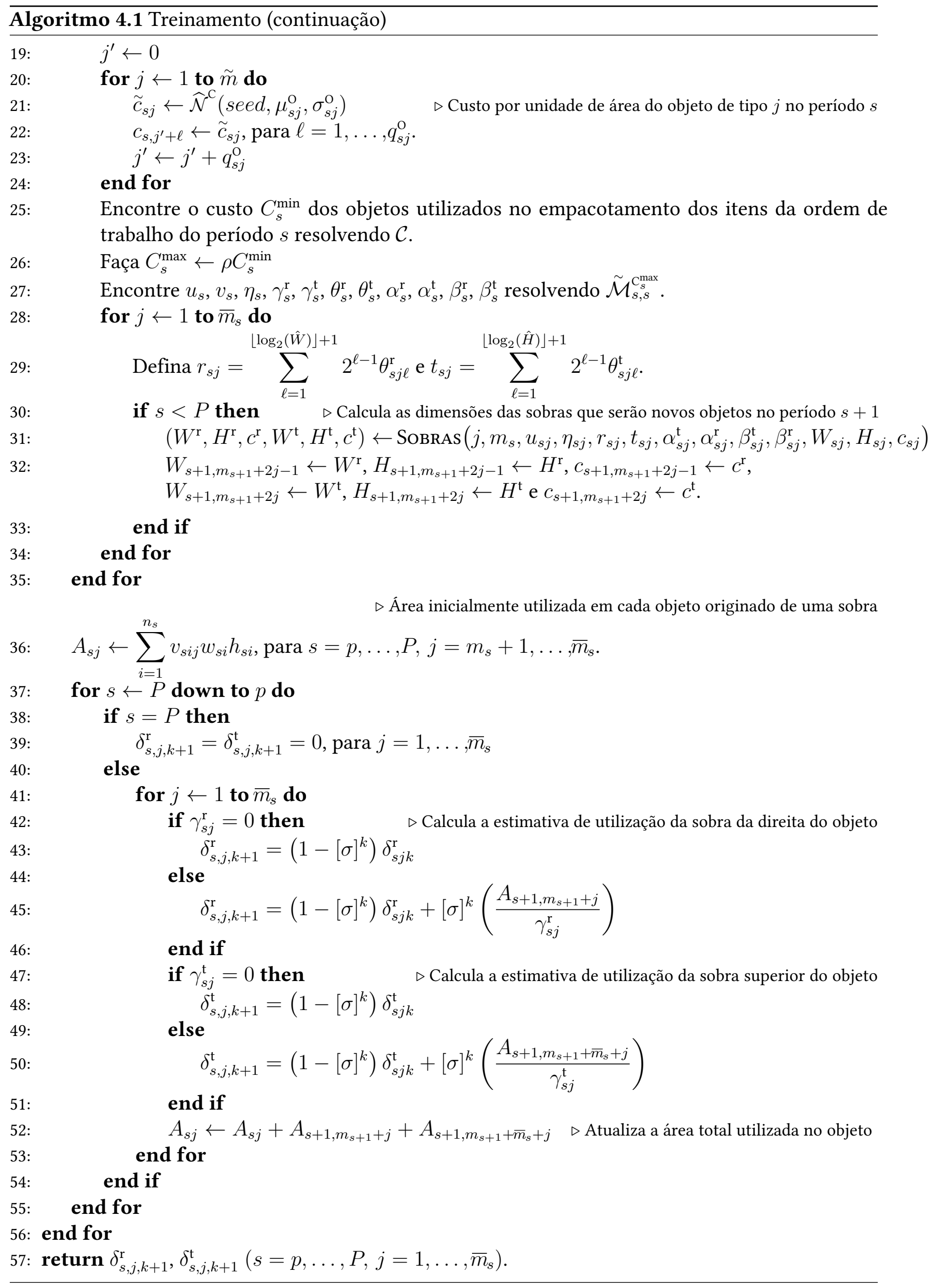


Com as estimativas de utilização futura das sobras ajustadas, podemos usar esses valores para resolver o problema considerando um cenário real (que segue as mesmas distribuições de probabilidade normal consideradas na fase de treinamento), onde os dados de cada período só são conhecidos no início do período, ou seja, no período $s$ apenas os dados desse período e dos anteriores são conhecidos. O Algoritmo 4.2 apresenta a fase de execução para resolver o problema usando as estimativas de proporção de utilização das sobras obtidas pelo Algoritmo 4.1. Além dos dados do problema, o Algoritmo 4.2 ainda recebe os valores das estimativas da proporção de utilização da sobra da direita $\left(\delta_{s j}^{\mathrm{r}}\right)$ e da sobra superior $\left(\delta_{s j}^{\mathrm{t}}\right)$ do objeto $j=1, \ldots, \bar{m}_{s}$ no período $s=p, \ldots, P$.

Como podemos perceber, o algoritmo apresenta um comportamento similar ao método guloso (Algoritmo 3.1): para cada período o algoritmo resolve o modelo $\tilde{\mathcal{M}}_{s, s}^{\mathrm{C}_{s}^{\text {max }}}$, mas, diferente do método guloso, utiliza as estimativas da proporção de utilização das sobras de cada objeto que foram ajustadas na fase de treinamento. Em seguida, as sobras são geradas e ficam disponíveis no período seguinte. Ao fim, o algoritmo retorna a solução global encontrada para o horizonte de planejamento. A diferença entre esse algoritmo e o método guloso está na formulação matemática que é resolvida. Enquanto o método guloso resolve o modelo $\mathcal{M}_{s, s}^{2}$, o Algoritmo 4.2 resolve o $\tilde{\mathcal{M}}_{s, s}^{\mathrm{c}_{s}^{\max }}$, que considera as informações adquiridas na fase de treinamento da PDA para tentar encontrar uma solução de melhor qualidade.

\section{Algoritmo 4.2 Execução}

Entrada: $p, P, \tilde{n}_{s}, n_{s}, w_{s i}, h_{s i}\left(s=p, \ldots, P, i=1, \ldots, n_{s}\right), d, \bar{w}_{i}, \bar{h}_{i}(i=1, \ldots, d), m_{s}, W_{s j}, H_{s j}$ $\left(s=p, \ldots, P, j=1, \ldots, m_{s}\right), \rho, \delta_{s j}^{\mathrm{r}}, \delta_{s j}^{\mathrm{t}}\left(s=p, \ldots, P, j=1, \ldots, \bar{m}_{s}\right)$.

Saída: $u_{s j}, \eta_{s j}, r_{s j}, t_{s j}\left(s=1, \ldots, P, j=1, \ldots, \bar{m}_{s}\right), v_{s i j}\left(s=p, \ldots, P, i=1, \ldots, n_{s}, j=1, \ldots, \bar{m}_{s}\right)$, $x_{s i}, y_{s i}\left(s=p, \ldots, P, i=1, \ldots, n_{s}\right), W_{s j}, H_{s j}\left(s=p, \ldots, P, j=1, \ldots, \bar{m}_{s}\right)$.

$\operatorname{Simulação~}\left(p, P, \tilde{n}_{s}, n_{s}, w_{s i}, h_{s i}, d, \bar{w}_{i}, \bar{h}_{i}, m_{s}, W_{s j}, H_{s j}, \rho, \delta_{s j}^{\mathrm{r}}, \delta_{s j}^{\mathrm{t}}\right)$.

1: Defina $\bar{m}_{s}=m_{s}+2\left(\sum_{i=1}^{\min (s-p, P+p-2)} 2^{i-1} m_{s-i}\right)$, para $s=p, \ldots, P$.

2: for $s \leftarrow p$ to $P$ do

3: $\quad$ Encontre o custo $C_{s}^{\mathrm{min}}$ dos objetos utilizados no empacotamento dos itens da ordem de trabalho do período $s$ resolvendo $\mathcal{C}$.

4: $\quad$ Faça $C_{s}^{\max } \leftarrow z C_{s}^{\min }$

5: $\quad$ Encontre $u_{s}, v_{s}, \eta_{s}, x_{s}, y_{s}, \gamma_{s}^{\mathrm{r}}, \gamma_{s}^{\mathrm{t}}, \theta_{s}^{\mathrm{r}}, \theta_{s}^{\mathrm{t}}, \alpha_{s}^{\mathrm{r}}, \alpha_{s}^{\mathrm{t}}, \beta_{s}^{\mathrm{r}}, \beta_{s}^{\mathrm{t}}$ resolvendo $\tilde{\mathcal{M}}_{s, s}^{\mathrm{C} \max }$ e considerando $\delta_{s j}^{\mathrm{r}}$ e $\delta_{s j}^{\mathrm{t}}$, para $j=1, \ldots, \bar{m}_{s}$.

6: $\quad$ for $j \leftarrow 1$ to $\bar{m}_{s}$ do

7: $\quad$ Defina $r_{s j}=\sum_{\ell=1}^{\left\lfloor\log _{2}(\hat{W})\right\rfloor+1} 2^{\ell-1} \theta_{s j \ell}^{\mathrm{r}}$ e $t_{s j}=\sum_{\ell=1}^{\left\lfloor\log _{2}(\hat{H})\right\rfloor+1} 2^{\ell-1} \theta_{s j \ell}^{\mathrm{t}}$.

8: $\quad$ if $s<P$ then $\quad \triangleright$ Calcula as dimensões das sobras que serão novos objetos no período $s+1$

9: $\quad\left(W^{\mathrm{r}}, H^{\mathrm{r}}, c^{\mathrm{r}}, W^{\mathrm{t}}, H^{\mathrm{t}}, c^{\mathrm{t}}\right) \leftarrow \operatorname{SobRAS}\left(j, m_{s}, u_{s j}, \eta_{s j}, r_{s j}, t_{s j}, \alpha_{s j}^{\mathrm{t}}, \alpha_{s j}^{\mathrm{r}}, \beta_{s j}^{\mathrm{t}}, \beta_{s j}^{\mathrm{r}}, W_{s j}, H_{s j}, c_{s j}\right)$

10: $\quad W_{s+1, m_{s+1}+2 j-1} \leftarrow W^{\mathrm{r}}, H_{s+1, m_{s+1}+2 j-1} \leftarrow H^{\mathrm{r}}, c_{s+1, m_{s+1}+2 j-1} \leftarrow c^{\mathrm{r}}$, $W_{s+1, m_{s+1}+2 j} \leftarrow W^{\mathrm{t}}, H_{s+1, m_{s+1}+2 j} \leftarrow H^{\mathrm{t}}$ e $c_{s+1, m_{s+1}+2 j} \leftarrow c^{\mathrm{t}}$.

11:

12:

end if

end for

13: end for

14: return $u_{s j}, \eta_{s j}, r_{s j}, t_{s j}\left(s=1, \ldots, P, j=1, \ldots, \bar{m}_{s}\right), v_{s i j}\left(s=p, \ldots, P, i=1, \ldots, n_{s}, j=1, \ldots, \bar{m}_{s}\right)$, $x_{s i}, y_{s i}\left(s=p, \ldots, P, i=1, \ldots, n_{s}\right), W_{s j}, H_{s j}, c_{s j}\left(s=p, \ldots, P, j=1, \ldots, \bar{m}_{s}\right)$. 
Antes de apresentarmos os experimentos numéricos, vamos descrever as características que um conjunto de problemas deve ter para que a PDA obtenha melhores resultados que o método guloso. Basicamente, duas características são necessárias:

1. Ao longo do horizonte rolante, deve existir itens de ordens de trabalho que utilizem todas ou a maioria das sobras aproveitáveis geradas. Como a PDA considera as estimativas do aproveitamento futuro das sobras de cada objeto, ao final do horizonte de planejamento é esperado que as sobras sejam, de fato, utilizadas para o corte de itens de ordens de trabalho futuras. Em outras palavras, devemos ter itens que caibam nas sobras geradas em períodos anteriores. Por exemplo, se no período $s$ as sobras possuem quase sempre dimensão $\dot{W} \times \dot{H}$, é desejável que nos períodos seguintes tenhamos uma quantidade suficiente de itens com dimensão $\dot{w} \times \dot{h}$, onde $\dot{w} \leq \dot{W}$ e $\dot{h} \leq \dot{H}$, que utilize a maior proporção possível das sobras geradas no período $s$, ou seja, a proporção de utilização das sobras é alta, indicando para a PDA que a compra de um ou mais objetos maiores e, possivelmente, mais caros, vale a pena. Por outro lado, se os itens de ordens de trabalho futuras são, em sua maioria, de dimensão $\dot{w} \times \dot{h}$, onde $\dot{w}>\dot{W}$ e $\dot{h}>\dot{H}$, então, ao fim do horizonte de planejamento, a maioria ou até todas as sobras não poderão ser utilizadas, o que indica para a PDA que não vale a pena investir na compra de objetos maiores;

2. O conjunto de objetos deve ser de tal forma que todos ou grande parte tenham a chance de serem selecionados. Isso significa que cada um dos objetos deve possuir o menor valor em (3.5) dependendo das estimativas da proporção de utilização das sobras. Caso contrário, apenas alguns objetos podem ser selecionados, enquanto outros nunca terão a chance de serem utilizados, ou seja, se alguns objetos sempre possuem valores maiores do que outros objetos em (3.5), independentemente das estimativas da proporção de utilização das sobras, esses objetos nunca serão selecionados. Note que se os objetos maiores forem mais baratos que os objetos menores, tanto o método guloso quanto a PDA se comportarão de forma similar, encontrando soluções iguais ou próximas. O mesmo acontece se a área de cada objeto disponível for muito próxima da área de qualquer outro.

\subsection{Experimentos computacionais}

Nesta seção, apresentamos experimentos computacionais para avaliar e comparar a abordagem baseada em programação dinâmica aproximada com a estratégia gulosa em diferentes cenários para a versão estocástica do problema. Como antes, todos os algoritmos foram implementados em $\mathrm{C}++\mathrm{e}$ compilados com GNU g++ versão 5.4 com a opção -03 ativada. A formulação dos modelos usados na PDA e no método guloso foi resolvida pelo IBM ILOG CPLEX versão 12.6.3, sendo implementada via ILOG Concert Technology. Com relação aos parâmetros do CPLEX, definimos a variável EpAGap para $10^{-7}$, EpGap para 0 e Threads para 1 . Todos os experimentos foram realizados em uma máquina Intel Xeon X5650 2.67GHz, com 8GB de memória RAM e Sistema Operacional Linux.

Geramos 7 conjuntos de problemas testes, descritos na Tabela 4.1, sendo que os cinco primeiros são conjuntos favoráveis à PDA (atendem as características para que a PDA obtenha bons resultados) 
e os dois últimos são conjuntos em que a PDA tende a não obter bons resultados. A ideia desses conjuntos é simular diferentes cenários com variações sazonais do custo dos objetos e das ordens de trabalho dos itens. Na tabela, na primeira coluna temos o número que identifica o conjunto, a coluna " $P$ " indica o número de períodos para os quais o conjunto possui informação. Para esses experimentos, consideramos que o período inicial $p$ é sempre 1. As três colunas seguintes estão associadas às informações dos objetos disponíveis para o corte dos itens ao longo do horizonte de planejamento. A coluna $\tilde{m}$ especifica o número de tipos de objetos disponíveis para a compra; a coluna $\tilde{W}_{j} \times \tilde{H}_{j}$ indica, respectivamente, a largura e a altura dos $\tilde{m}$ objetos; e a coluna $\left(\mu_{s j}^{\mathrm{O}} ; \sigma_{s j}^{\mathrm{O}}\right)$ indica a média e o desvio padrão do custo por unidade de área de cada objeto para os $P$ períodos. As próximas quatro colunas referem-se às informações dos itens das ordens de trabalho. A coluna $\tilde{n}$ informa a quantidade de tipo de itens que podem ser solicitado; a coluna $d$ especifica a quantidade de itens do catálogo; a coluna $\tilde{w}_{i} \times \tilde{h}_{i}$ indica a largura e a altura, respectivamente, de cada um dos $\tilde{n}$ itens; e a coluna $\left(\mu_{s i}^{\mathrm{I}} ; \sigma_{s i}^{\mathrm{I}}\right)$ especifica a média e o desvio padrão da quantidade de cada item para os $P$ períodos. Consideramos os itens sublinhados como sendo os itens do catálogo.

Gerando amostras com as características de cada conjunto, ajustamos as estimativas da proporção de aproveitamento das sobras dos objetos da PDA, usando o algoritmo de treinamento (Algoritmo 4.1), com os seguinte parâmetros: $\sigma \in\{0,1 ; 0,5 ; 0,8 ; 0,9\}, \delta_{\text {ini }} \in\{0,10 ; 0,20 ; 0,30 ; 0,40 ; 0,50 ; 0,60 ; 0,70 ; 0,80$; $0,90 ; 1,00\}$ e $\rho=2,0$. Para o critério de parada do Algoritmo 4.1, definimos $\epsilon=0,01$ na equação (3.7). Uma vez ajustadas as estimativas da proporção de aproveitamento das sobras dos objetos, geramos 30 instâncias para cada conjunto de problemas testes, com as mesmas características contidas no conjunto, e cada instância gerada é resolvida pela PDA usando os parâmetros encontrados na fase de treinamento. A fim de avaliar a qualidade da solução encontrada pela PDA, cada instância gerada também é resolvida usando o método guloso (Algoritmo 3.1). Como métrica para avaliar a qualidade das soluções encontradas pela PDA em relação as soluções obtidas pelo método guloso, usamos a seguinte medida:

$$
\text { Melhora }=\frac{f_{\text {guloso }}-f_{\text {pda }}}{f_{\text {guloso }}} \times 100 \%,
$$

onde $f_{\text {pda }}$ é a solução obtida pelo Algoritmo 4.1 e $f_{\text {guloso }}$ é a solução obtida pelo método guloso (Algoritmo 3.1). Essa medida indica, em porcentagem, o quão melhor (ou quão pior) a PDA foi em relação ao método guloso. Assim, um valor positivo indica que a PDA obteve uma solução melhor que o método guloso e um valor negativo indica o contrário. 
Tabela 4.1: Dados dos conjuntos de problemas testes

\begin{tabular}{|c|c|c|c|c|c|c|c|c|}
\hline \multirow{2}{*}{ Conjunto } & \multirow{2}{*}{$P$} & \multicolumn{3}{|c|}{ Objetos } & \multicolumn{4}{|c|}{ Itens } \\
\hline & & $\tilde{m}$ & $\tilde{W}_{j} \times \tilde{H}_{j}$ & $\left(\mu_{s j}^{\mathrm{O}} ; \sigma_{s j}^{\mathrm{O}}\right)$ & $\tilde{n}$ & $d$ & $\tilde{w}_{i} \times \tilde{h}_{i}$ & $\left(\mu_{s i}^{\mathrm{I}} ; \sigma_{s i}^{\mathrm{I}}\right)$ \\
\hline \multirow[t]{8}{*}{1} & 4 & 2 & $7 \times 5$ & $(0,98 ; 0,01)$ & 1 & 1 & $\underline{2 \times 2}$ & $(4,27 ; 0,21)$ \\
\hline & & & & $(1,01 ; 0,02)$ & & & & $(8,06 ; 0,28)$ \\
\hline & & & & $(0,99 ; 0,01)$ & & & & $(5,33 ; 0,12)$ \\
\hline & & & & $(1,03 ; 0,02)$ & & & & $(3,54 ; 0,62)$ \\
\hline & & & $6 \times 6$ & $(1,02 ; 0,01)$ & & & & \\
\hline & & & & $(1,08 ; 0,01)$ & & & & \\
\hline & & & & $(1,05 ; 0,01)$ & & & & \\
\hline & & & & $(1,02 ; 0,02)$ & & & & \\
\hline \multirow[t]{16}{*}{2} & 8 & 2 & $7 \times 5$ & $(1,02 ; 0,02)$ & 1 & 1 & $2 \times 2$ & $(2,08 ; 0,22)$ \\
\hline & & & & $(1,05 ; 0,02)$ & & & & $(2,01 ; 0,35)$ \\
\hline & & & & $(1,06 ; 0,03)$ & & & & $(1,94 ; 0,29)$ \\
\hline & & & & $(1,07 ; 0,02)$ & & & & $(2,27 ; 0,23)$ \\
\hline & & & & $(1,38 ; 0,03)$ & & & & $(5,31 ; 0,31)$ \\
\hline & & & & $(1,76 ; 0,03)$ & & & & $(8,94 ; 0,28)$ \\
\hline & & & & $(1,29 ; 0,03)$ & & & & $(4,91 ; 0,32)$ \\
\hline & & & & $(1,03 ; 0,02)$ & & & & $(2,16 ; 0,16)$ \\
\hline & & & $8 \times 6$ & $(0,85 ; 0,03)$ & & & & \\
\hline & & & & $(0,83 ; 0,02)$ & & & & \\
\hline & & & & $(0,87 ; 0,02)$ & & & & \\
\hline & & & & $(0,86 ; 0,02)$ & & & & \\
\hline & & & & $(1,13 ; 0,03)$ & & & & \\
\hline & & & & $(1,39 ; 0,03)$ & & & & \\
\hline & & & & $(1,06 ; 0,02)$ & & & & \\
\hline & & & & $(0,83 ; 0,03)$ & & & & \\
\hline
\end{tabular}


Tabela 4.1 - continuação da página anterior

\begin{tabular}{|c|c|c|c|c|c|c|c|c|}
\hline \multirow{2}{*}{ Conjunto } & \multirow{2}{*}{$P$} & \multicolumn{3}{|c|}{ Objetos } & \multicolumn{4}{|c|}{ Itens } \\
\hline & & $\tilde{m}$ & $\tilde{W}_{j} \times \tilde{H}_{j}$ & $\left(\mu_{s j}^{\mathrm{O}} ; \sigma_{s j}^{\mathrm{O}}\right)$ & $\tilde{n}$ & $d$ & $\tilde{w}_{i} \times \tilde{h}_{i}$ & $\left(\mu_{s i}^{\mathrm{I}} ; \sigma_{s i}^{\mathrm{I}}\right)$ \\
\hline \multirow[t]{25}{*}{3} & 8 & 2 & $9 \times 7$ & $(0,98 ; 0,01)$ & 3 & 1 & $4 \times 3$ & $(1,13 ; 0,11)$ \\
\hline & & & & $(1,06 ; 0,02)$ & & & & $(2,07 ; 0,09)$ \\
\hline & & & & $(1,26 ; 0,02)$ & & & & $(2,05 ; 0,12)$ \\
\hline & & & & $(1,17 ; 0,01)$ & & & & $(1,51 ; 0,31)$ \\
\hline & & & & $(1,09 ; 0,03)$ & & & & $(1,03 ; 0,13)$ \\
\hline & & & & $(1,47 ; 0,02)$ & & & & $(1,43 ; 0,29)$ \\
\hline & & & & $(1,63 ; 0,02)$ & & & & $(3,07 ; 0,09)$ \\
\hline & & & & $(1,67 ; 0,03)$ & & & & $(0,92 ; 0,17)$ \\
\hline & & & $10 \times 9$ & $(0,74 ; 0,01)$ & & & $5 \times 2$ & $(1,23 ; 0,07)$ \\
\hline & & & & $(0,83 ; 0,01)$ & & & & $(1,13 ; 0,11)$ \\
\hline & & & & $(1,03 ; 0,02)$ & & & & $(1,47 ; 0,21)$ \\
\hline & & & & $(0,89 ; 0,01)$ & & & & $(1,47 ; 0,23)$ \\
\hline & & & & $(0,86 ; 0,01)$ & & & & $(0,82 ; 0,19)$ \\
\hline & & & & $(1,18 ; 0,03)$ & & & & $(1,37 ; 0,24)$ \\
\hline & & & & $(1,28 ; 0,02)$ & & & & $(1,51 ; 0,31)$ \\
\hline & & & & $(1,32 ; 0,02)$ & & & & $(0,31 ; 0,09)$ \\
\hline & & & & & & & $\underline{2 \times 2}$ & $(7,53 ; 0,51)$ \\
\hline & & & & & & & & $(4,36 ; 0,38)$ \\
\hline & & & & & & & & $(3,39 ; 0,27)$ \\
\hline & & & & & & & & $(6,15 ; 0,10)$ \\
\hline & & & & & & & & $(8,10 ; 0,12)$ \\
\hline & & & & & & & & $(3,56 ; 0,17)$ \\
\hline & & & & & & & & $(0,25 ; 0,06)$ \\
\hline & & & & & & & & $(6,07 ; 0,14)$ \\
\hline & & & & & & & na & na página \\
\hline
\end{tabular}


Tabela 4.1 - continuação da página anterior

\begin{tabular}{|c|c|c|c|c|c|c|c|c|}
\hline \multirow{2}{*}{ Conjunto } & \multirow{2}{*}{$P$} & \multicolumn{3}{|c|}{ Objetos } & \multicolumn{4}{|c|}{ Itens } \\
\hline & & $\tilde{m}$ & $\tilde{W}_{j} \times \tilde{H}_{j}$ & $\left(\mu_{s j}^{\mathrm{O}} ; \sigma_{s j}^{\mathrm{O}}\right)$ & $\tilde{n}$ & $d$ & $\tilde{w}_{i} \times \tilde{h}_{i}$ & $\left(\mu_{s i}^{\mathrm{I}} ; \sigma_{s i}^{\mathrm{I}}\right)$ \\
\hline \multirow[t]{40}{*}{4} & 8 & 2 & $15 \times 10$ & $(0,97 ; 0,02)$ & 5 & 1 & $4 \times 6$ & $(1,76 ; 0,22)$ \\
\hline & & & & $(1,01 ; 0,02)$ & & & & $(1,13 ; 0,29)$ \\
\hline & & & & $(1,23 ; 0,01)$ & & & & $(0,71 ; 0,12)$ \\
\hline & & & & $(1,48 ; 0,02)$ & & & & $(0,40 ; 0,09)$ \\
\hline & & & & $(1,07 ; 0,03)$ & & & & $(1,37 ; 0,11)$ \\
\hline & & & & $(1,21 ; 0,01)$ & & & & $(1,09 ; 0,09)$ \\
\hline & & & & $(1,46 ; 0,03)$ & & & & $(2,71 ; 0,12)$ \\
\hline & & & & $(1,86 ; 0,02)$ & & & & $(0,46 ; 0,10)$ \\
\hline & & & $20 \times 10$ & $(0,82 ; 0,01)$ & & & $5 \times 5$ & $(1,78 ; 0,19)$ \\
\hline & & & & $(0,87 ; 0,02)$ & & & & $(0,66 ; 0,19)$ \\
\hline & & & & $(0,99 ; 0,01)$ & & & & $(2,73 ; 0,23)$ \\
\hline & & & & $(1,25 ; 0,02)$ & & & & $(0,61 ; 0,07)$ \\
\hline & & & & $(0,96 ; 0,02)$ & & & & $(1,03 ; 0,08)$ \\
\hline & & & & $(1,02 ; 0,02)$ & & & & $(1,63 ; 0,13)$ \\
\hline & & & & $(1,27 ; 0,01)$ & & & & $(0,46 ; 0,13)$ \\
\hline & & & & $(1,53 ; 0,02)$ & & & & $(0,35 ; 0,07)$ \\
\hline & & & & & & & $3 \times 2$ & $(1,47 ; 0,28)$ \\
\hline & & & & & & & & $(3,63 ; 0,17)$ \\
\hline & & & & & & & & $(1,37 ; 0,30)$ \\
\hline & & & & & & & & $(5,42 ; 0,35)$ \\
\hline & & & & & & & & $(3,29 ; 0,37)$ \\
\hline & & & & & & & & $(2,59 ; 0,09)$ \\
\hline & & & & & & & & $(2,22 ; 0,10)$ \\
\hline & & & & & & & & $(6,69 ; 0,59)$ \\
\hline & & & & & & & $3 \times 4$ & $(1,74 ; 0,24)$ \\
\hline & & & & & & & & $(0,59 ; 0,13)$ \\
\hline & & & & & & & & $(0,66 ; 0,18)$ \\
\hline & & & & & & & & $(3,55 ; 0,41)$ \\
\hline & & & & & & & & $(1,92 ; 0,12)$ \\
\hline & & & & & & & & $(2,64 ; 0,10)$ \\
\hline & & & & & & & & $(3,11 ; 0,12)$ \\
\hline & & & & & & & & $(5,19 ; 0,36)$ \\
\hline & & & & & & & $2 \times 2$ & $(1,83 ; 0,52)$ \\
\hline & & & & & & & & $(7,38 ; 0,34)$ \\
\hline & & & & & & & & $(2,38 ; 0,63)$ \\
\hline & & & & & & & & $(7,30 ; 0,37)$ \\
\hline & & & & & & & & $(4,33 ; 0,23)$ \\
\hline & & & & & & & & $(4,02 ; 0,15)$ \\
\hline & & & & & & & & $(5,29 ; 0,37)$ \\
\hline & & & & & & & & $(6,65 ; 0,43)$ \\
\hline
\end{tabular}


Tabela 4.1 - continuação da página anterior

\begin{tabular}{|c|c|c|c|c|c|c|c|c|}
\hline \multirow{2}{*}{ Conjunto } & \multirow{2}{*}{$P$} & \multicolumn{3}{|c|}{ Objetos } & \multicolumn{4}{|c|}{ Itens } \\
\hline & & $\tilde{m}$ & $\tilde{W}_{j} \times \tilde{H}_{j}$ & $\left(\mu_{s j}^{\mathrm{O}} ; \sigma_{s j}^{\mathrm{O}}\right)$ & $\tilde{n}$ & $d$ & $\tilde{w}_{i} \times \tilde{h}_{i}$ & $\left(\mu_{s i}^{\mathrm{I}} ; \sigma_{s i}^{\mathrm{I}}\right)$ \\
\hline \multirow[t]{20}{*}{5} & 4 & 3 & $20 \times 10$ & $(1,02 ; 0,02)$ & 5 & 1 & $5 \times 6$ & $(1,09 ; 0,11)$ \\
\hline & & & & $(1,37 ; 0,02)$ & & & & $(0,29 ; 0,06)$ \\
\hline & & & & $(1,28 ; 0,01)$ & & & & $(1,13 ; 0,12)$ \\
\hline & & & & $(2,04 ; 0,02)$ & & & & $(0,34 ; 0,06)$ \\
\hline & & & $15 \times 15$ & $(1,00 ; 0,01)$ & & & $5 \times 4$ & $(1,82 ; 0,19)$ \\
\hline & & & & $(1,34 ; 0,02)$ & & & & $(0,27 ; 0,07)$ \\
\hline & & & & $(1,24 ; 0,01)$ & & & & $(1,19 ; 0,23)$ \\
\hline & & & & $(1,98 ; 0,02)$ & & & & $(0,33 ; 0,07)$ \\
\hline & & & $20 \times 15$ & $(0,86 ; 0,01)$ & & & $3 \times 4$ & $(3,33 ; 0,07)$ \\
\hline & & & & $(1,09 ; 0,01)$ & & & & $(1,48 ; 0,09)$ \\
\hline & & & & $(1,04 ; 0,02)$ & & & & $(2,81 ; 0,19)$ \\
\hline & & & & $(1,63 ; 0,01)$ & & & & $(1,66 ; 0,23)$ \\
\hline & & & & & & & $3 \times 3$ & $(2,11 ; 0,39)$ \\
\hline & & & & & & & & $(1,64 ; 0,21)$ \\
\hline & & & & & & & & $(3,77 ; 0,21)$ \\
\hline & & & & & & & & $(1,73 ; 0,17)$ \\
\hline & & & & & & & $6 \times 3$ & $(0,30 ; 0,08)$ \\
\hline & & & & & & & & $(2,71 ; 0,15)$ \\
\hline & & & & & & & & $(1,89 ; 0,13)$ \\
\hline & & & & & & & & $(1,41 ; 0,21)$ \\
\hline \multirow[t]{20}{*}{6} & 4 & 3 & $20 \times 10$ & $(1,02 ; 0,02)$ & 5 & 1 & $5 \times 6$ & $(1,09 ; 0,11)$ \\
\hline & & & & $(1,37 ; 0,02)$ & & & & $(1,19 ; 0,13)$ \\
\hline & & & & $(1,28 ; 0,01)$ & & & & $(1,13 ; 0,12)$ \\
\hline & & & & $(2,04 ; 0,02)$ & & & & $(1,53 ; 0,24)$ \\
\hline & & & $15 \times 15$ & $(1,00 ; 0,01)$ & & & $5 \times 4$ & $(1,82 ; 0,19)$ \\
\hline & & & & $(1,34 ; 0,02)$ & & & & $(0,66 ; 0,11)$ \\
\hline & & & & $(1,24 ; 0,01)$ & & & & $(1,19 ; 0,23)$ \\
\hline & & & & $(1,98 ; 0,02)$ & & & & $(0,87 ; 0,19)$ \\
\hline & & & $20 \times 15$ & $(0,86 ; 0,01)$ & & & $3 \times 4$ & $(3,33 ; 0,07)$ \\
\hline & & & & $(1,09 ; 0,01)$ & & & & $(1,48 ; 0,09)$ \\
\hline & & & & $(1,04 ; 0,02)$ & & & & $(2,81 ; 0,19)$ \\
\hline & & & & $(1,63 ; 0,01)$ & & & & $(1,66 ; 0,23)$ \\
\hline & & & & & & & $3 \times 3$ & $(2,11 ; 0,39)$ \\
\hline & & & & & & & & $(1,64 ; 0,21)$ \\
\hline & & & & & & & & $(3,77 ; 0,21)$ \\
\hline & & & & & & & & $(1,73 ; 0,17)$ \\
\hline & & & & & & & $6 \times 3$ & $(0,30 ; 0,08)$ \\
\hline & & & & & & & & $(2,71 ; 0,15)$ \\
\hline & & & & & & & & $(1,89 ; 0,13)$ \\
\hline & & & & & & & & $(1,41 ; 0,21)$ \\
\hline
\end{tabular}


Tabela 4.1 - continuação da página anterior

\begin{tabular}{|c|c|c|c|c|c|c|c|c|}
\hline \multirow{2}{*}{ Conjunto } & \multirow{2}{*}{$P$} & \multicolumn{3}{|c|}{ Objetos } & \multicolumn{4}{|c|}{ Itens } \\
\hline & & $\tilde{m}$ & $\tilde{W}_{j} \times \tilde{H}_{j}$ & $\left(\mu_{s j}^{\mathrm{O}} ; \sigma_{s j}^{\mathrm{O}}\right)$ & $\tilde{n}$ & $d$ & $\tilde{w}_{i} \times \tilde{h}_{i}$ & $\left(\mu_{s i}^{\mathrm{I}} ; \sigma_{s i}^{\mathrm{I}}\right)$ \\
\hline \multirow[t]{20}{*}{7} & 4 & 3 & $20 \times 10$ & $(1,01 ; 0,02)$ & 5 & 1 & $5 \times 6$ & $(1,09 ; 0,11)$ \\
\hline & & & & $(1,13 ; 0,03)$ & & & & $(0,29 ; 0,06)$ \\
\hline & & & & $(1,09 ; 0,02)$ & & & & $(1,13 ; 0,12)$ \\
\hline & & & & $(1,12 ; 0,03)$ & & & & $(0,34 ; 0,06)$ \\
\hline & & & $15 \times 15$ & $(1,03 ; 0,02)$ & & & $5 \times 4$ & $(1,82 ; 0,19)$ \\
\hline & & & & $(1,15 ; 0,02)$ & & & & $(0,27 ; 0,07)$ \\
\hline & & & & $(1,11 ; 0,04)$ & & & & $(1,19 ; 0,23)$ \\
\hline & & & & $(1,17 ; 0,02)$ & & & & $(0,33 ; 0,07)$ \\
\hline & & & $20 \times 15$ & $(1,01 ; 0,03)$ & & & $3 \times 4$ & $(3,33 ; 0,07)$ \\
\hline & & & & $(1,11 ; 0,03)$ & & & & $(1,48 ; 0,09)$ \\
\hline & & & & $(1,07 ; 0,05)$ & & & & $(2,81 ; 0,19)$ \\
\hline & & & & $(1,13 ; 0,04)$ & & & & $(1,66 ; 0,23)$ \\
\hline & & & & & & & $3 \times 3$ & $(2,11 ; 0,39)$ \\
\hline & & & & & & & & $(1,64 ; 0,21)$ \\
\hline & & & & & & & & $(3,77 ; 0,21)$ \\
\hline & & & & & & & & $(1,73 ; 0,17)$ \\
\hline & & & & & & & $6 \times 3$ & $(0,30 ; 0,08)$ \\
\hline & & & & & & & & $(2,71 ; 0,15)$ \\
\hline & & & & & & & & $(1,89 ; 0,13)$ \\
\hline & & & & & & & & $(1,41 ; 0,21)$ \\
\hline
\end{tabular}

Inicialmente, para ilustrar como a PDA funciona durante a fase de treinamento, o gráfico da Figura 4.1 mostra o valor da função objetivo em cada iteração na fase de treinamento, considerando $\delta_{\text {ini }} \in\{0,10 ; 0,50 ; 0,80 ; 1,00\}$ e $\sigma=0,9$, para o Conjunto 2. Podemos notar que a PDA com $\delta_{\text {ini }}=0,10$ encontra soluções de maior custo, em quase todas as iterações, enquanto a PDA com $\delta_{\text {ini }}=0,80$ obtém, quase sempre, soluções de menor custo, indicando que o método, com $\delta_{\text {ini }}=0,80$, aprende mais rapidamente como melhorar a qualidade das soluções nas amostras geradas. Como veremos adiante, a PDA com $\delta_{\text {ini }}=0,80$ encontra, de fato, soluções melhores que a PDA com $\delta_{\text {ini }}=0,10$ quando consideramos o Conjunto 2. As duas primeiras iterações da PDA com $\delta_{\text {ini }}=1,00$ possuem custo muito elevado, isso porque esse $\delta_{\text {ini }}$ irá selecionar, nas primeiras iterações, uma grande quantidade de objetos.

Para o primeiro conjunto temos um horizonte de planejamento com quatro períodos, dois tipos de objetos (com dimensões $7 \times 5$ e $6 \times 6$ ) e um único tipo de item (de dimensão $2 \times 2$ ). Perceba que nesse conjunto há uma pequena variação nos preços dos objetos ao longo dos 4 períodos, mas o objeto $7 \times 5$ é sempre ligeiramente mais barato que o objeto $6 \times 6$, o que faz com que o método guloso escolha, sempre que possível e necessário, o objeto $7 \times 5$. Entretanto, observe que as possíveis sobras do objeto $6 \times 6$ podem ser melhor utilizadas, uma vez que possuem largura e altura múltiplas da largura e altura do item $2 \times 2$. Em relação à quantidade de itens em cada período, note que apenas 
no segundo período há uma quantidade média maior que nos outros períodos (que é, em média, 4 itens).

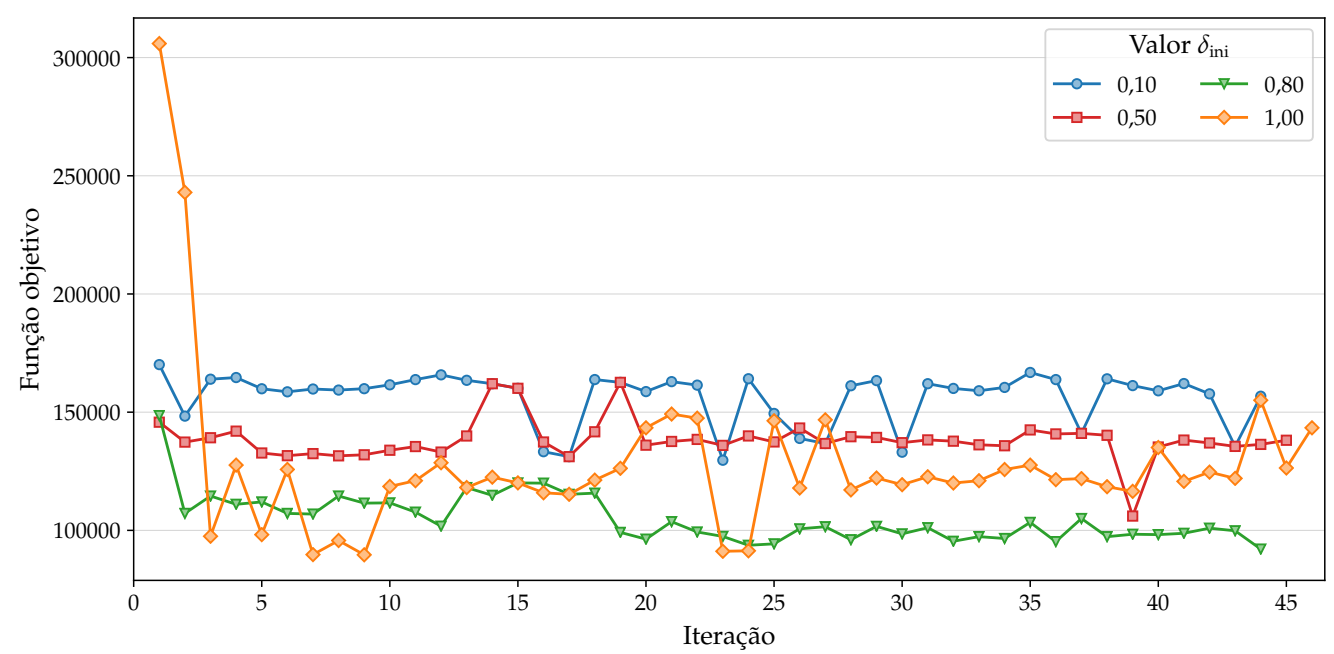

Figura 4.1: Valor da função objetivo, para o Conjunto 2, durante a fase de treinamento da PDA com $\sigma=0,9$ alguns valores de $\delta_{\text {ini. }}$.

O gráfico da Figura 4.2 mostra o logaritmo do tempo gasto, em segundos, na fase de treinamento da PDA para os diferentes valores de $\sigma$ e $\delta_{\text {ini. }}$. Como podemos observar, na maioria dos casos, quanto maior o valor de $\sigma$ ou $\delta_{\text {ini }}$, maior o tempo gasto. Isso porque o valor de $\sigma$ está diretamente ligado ao número de iterações necessárias para o algoritmo convergir e $\delta_{\text {ini }}$ está associado à quantidade de objetos que têm a chance de serem utilizados, fazendo com que os objetos mais caros tenham poucas (ou nenhuma) chances de serem utilizados quanto menor o valor de $\delta_{\text {ini }}$.

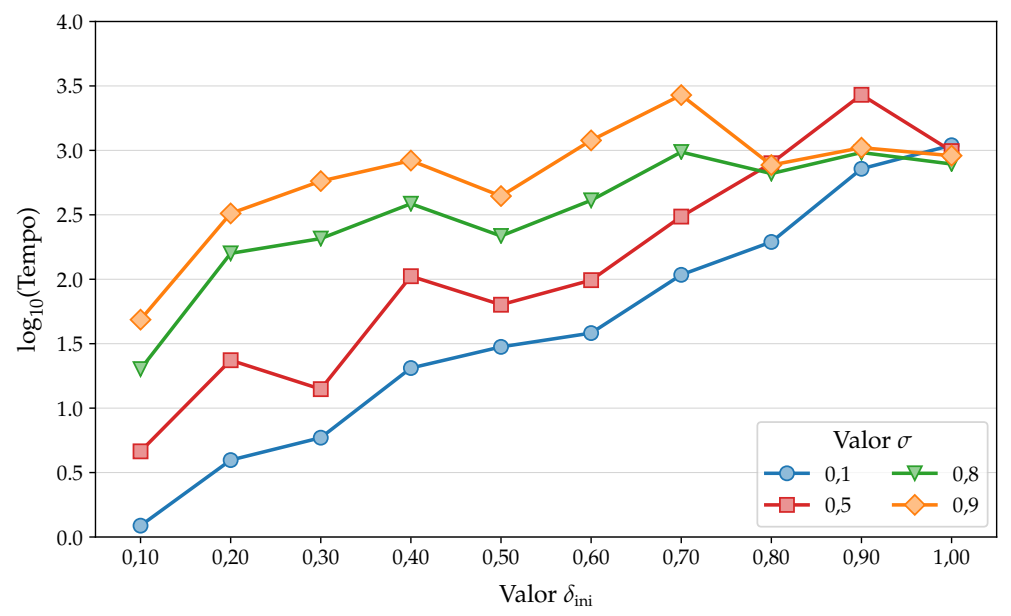

Figura 4.2: Tempo gasto, em escala logarítmica, na fase de treinamento para a PDA no Conjunto 1 para cada combinação de valor de $\delta_{\text {ini }}$ e $\sigma$.

O gráfico da Figura 4.3 resume os resultados obtidos pela PDA em comparação com o método guloso, para os diferentes valores de $\sigma$ e $\delta_{\text {ini }}$, considerando os dados do Conjunto 1. Como podemos observar, quando $\sigma \in\{0,1 ; 0,5\}$ e $\delta_{\text {ini }}=0,10$ a PDA não obteve bons resultados, sendo, em média, $0,36 \%$ pior que o método guloso (veja a Figura 4.4). Em ambos os casos, apenas em 4 instâncias a PDA 
obteve melhores resultados que o método guloso, como podemos ver na Tabela 4.2. Quando $\sigma=0,1$ e $\delta_{\text {ini }}=0,20$ a PDA obteve soluções com maior variabilidade. Para todas as outras combinações, a variabilidade foi bem pequena. Praticamente todas as combinações obtiveram outliers superiores, mas quando $\delta_{\text {ini }} \in\{0,70 ; 0,80 ; 1,00\}$, independente do valor de $\sigma$, não houveram outliers inferiores. Para esses valores, a PDA encontrou soluções melhores que o método guloso para as 30 instâncias consideradas, veja a Tabela 4.2. Como podemos ver pela Figura 4.4, a PDA obteve soluções com melhora média próxima de $20 \%$ para $\delta_{\text {ini }} \in\{0,30 ; 0,40 ; 0,50 ; 0,60 ; 0,70 ; 0,80 ; 1,00\}$, independente do valor de $\sigma$, sendo a maior melhora média $(21,55 \%)$ quando $\sigma=0,5$ e $\delta_{\text {ini }}=0,90$.

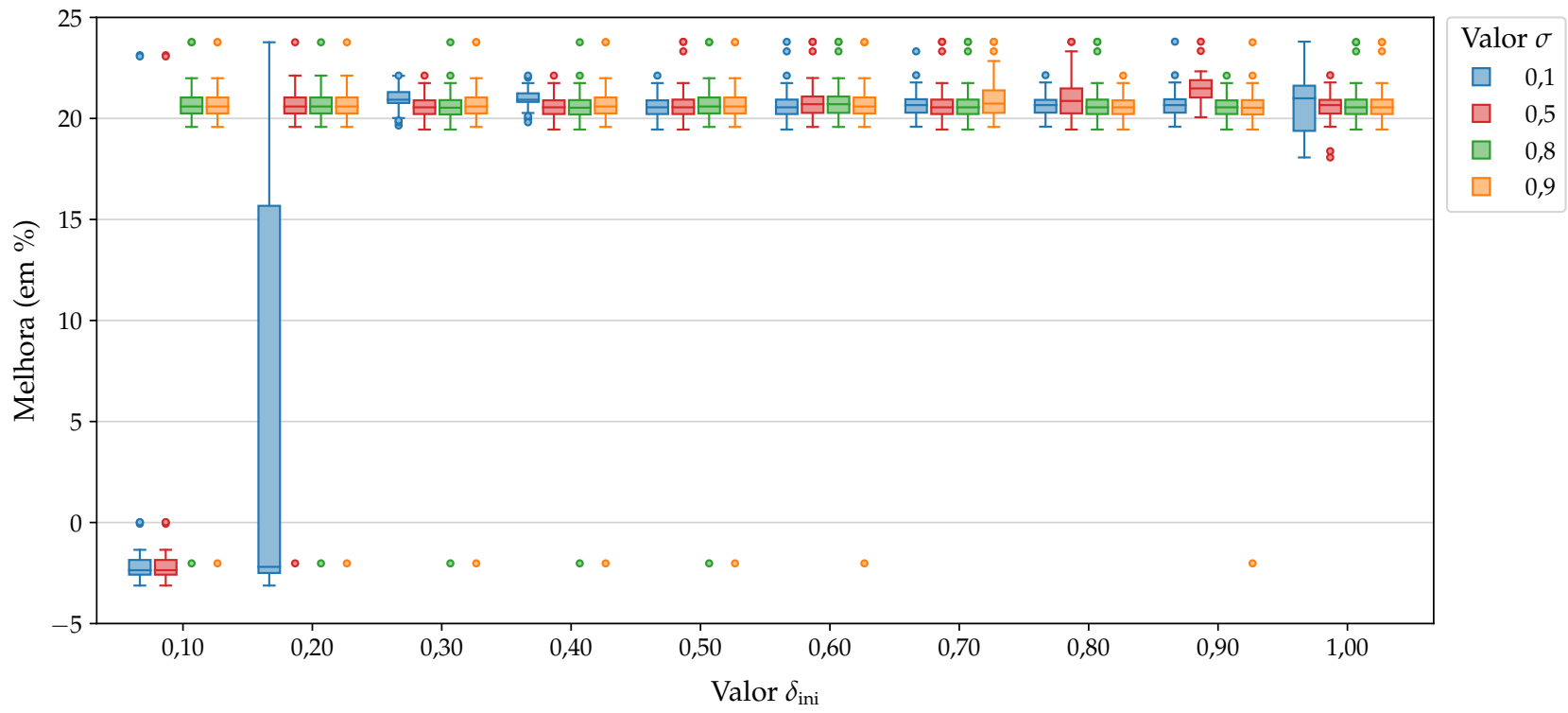

Figura 4.3: Resumo dos resultados encontrados pela PDA em comparação com o método guloso para o Conjunto 1.

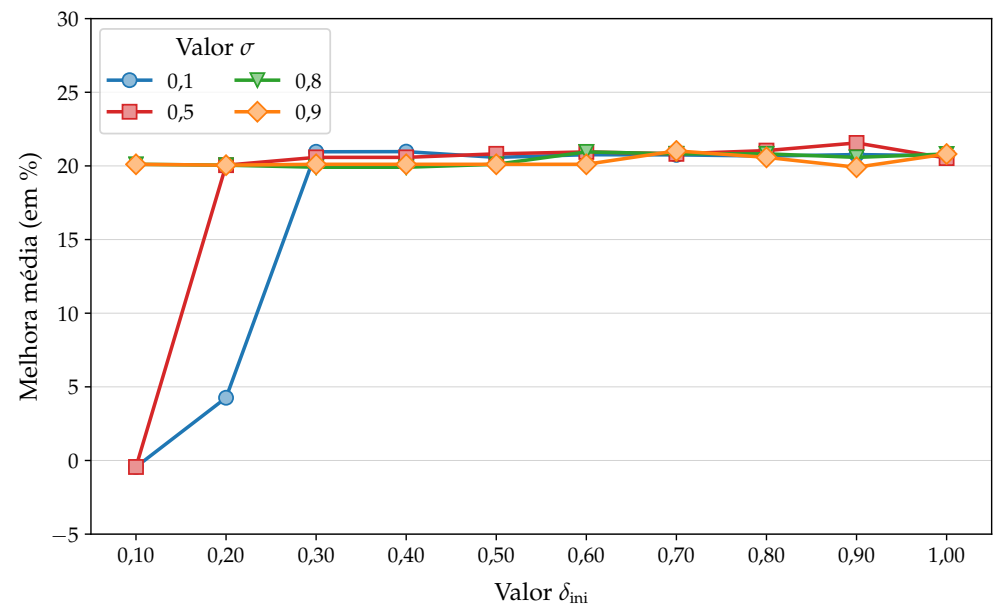

Figura 4.4: Melhora média (em porcentagem) para cada combinação de $\delta_{\text {ini }}$ e $\sigma$ para a PDA em relação ao método guloso, considerando o Conjunto 1. 
Tabela 4.2: Número de soluções encontradas pela PDA que são melhores que as obtidas pelo método guloso para o Conjunto 1 .

\begin{tabular}{rrrrrrrrrrrrr}
\hline$\sigma$ & \multicolumn{10}{c}{$\delta_{\text {ini }}$} & \multirow{1}{*}{ Média } \\
\cline { 2 - 9 } & 0,10 & 0,20 & 0,30 & 0,40 & 0,50 & 0,60 & 0,70 & 0,80 & 0,90 & 1,00 & \\
\hline 0,1 & 4 & 9 & 30 & 30 & 30 & 30 & 30 & 30 & 30 & 30 & 25,30 \\
0,5 & 4 & 29 & 30 & 30 & 30 & 30 & 30 & 30 & 30 & 30 & 27,30 \\
0,8 & 29 & 29 & 29 & 29 & 29 & 30 & 30 & 30 & 30 & 30 & 29,50 \\
0,9 & 29 & 29 & 29 & 29 & 29 & 29 & 30 & 30 & 29 & 30 & 29,30 \\
\hline Média & 16,50 & 24,00 & 29,50 & 29,50 & 29,50 & 29,75 & 30,00 & 30,00 & 29,75 & 30,00 & 27,85 \\
\hline
\end{tabular}

O segundo conjunto de problemas testes possui 8 períodos, dois tipos de objetos e um tipo de item. Em cada período, o objeto $8 \times 6$ (de maior área) possui custo, em média, próximo do custo do objeto $7 \times 5$ (veja a Figura 4.5), de forma que o custo "artificial" do objeto $8 \times 6$ (custo do objeto menos o valor das sobras aproveitáveis do objeto multiplicado pelas estimativas da proporção de utilização das sobras) pode ser menor ou igual que o mesmo custo "artificial" do objeto $7 \times 5$, dependendo do valor das sobras de cada objeto. O que permite que ambos os objetos tenham chances de serem escolhidos pela PDA, dependendo dos valores das estimativas de proporção de aproveitamento das sobras. Note que o preço dos objetos, em média, é praticamente constante nos quatro primeiros períodos, cresce nos dois períodos seguintes e volta a diminuir nos outros dois períodos, como mostra a Figura 4.5. O mesmo também ocorre com a quantidade de itens solicitados (veja a coluna $\left(\mu_{s i}^{\mathrm{I}} ; \sigma_{s i}^{\mathrm{I}}\right)$ do Conjunto 2 na Tabela 4.1). Assim, é desejável que nos períodos onde o custo médio é maior tenhamos uma quantidade de sobras suficientes para comprar o menor número de objetos possíveis e necessários para atender a ordem de trabalho dos itens nesses períodos.

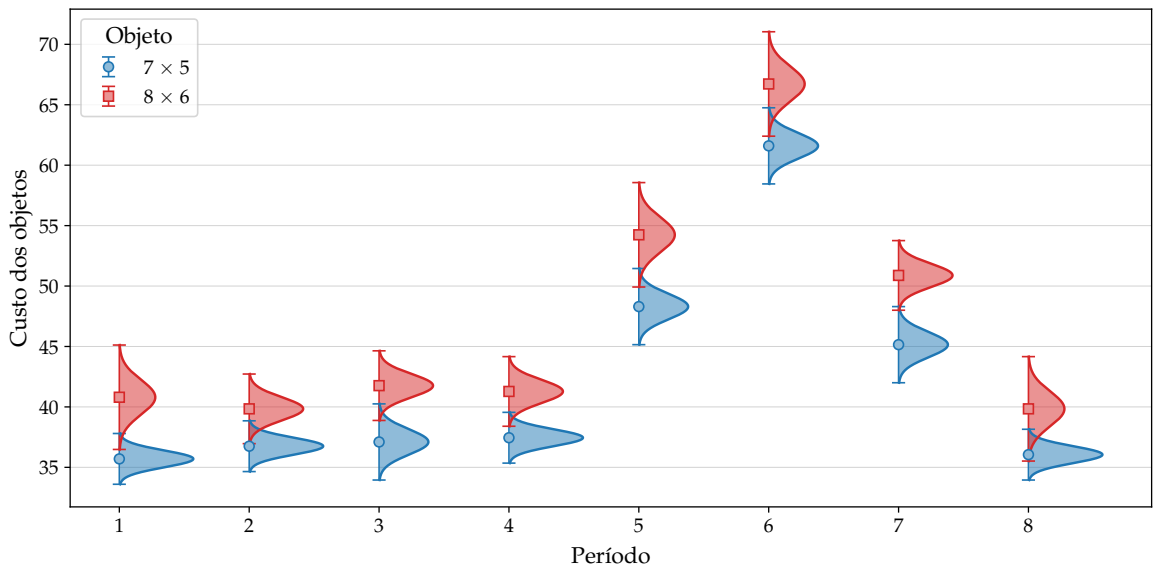

Figura 4.5: Custo mínimo, médio e máximo dos dois objetos, em cada período, do Conjunto 2. O custo máximo do objeto $j$ no período $s$ é calculado como $\mu_{s i}^{\mathrm{O}}+3 \times \sigma_{s i}^{\mathrm{O}}$ e o custo mínimo como $\mu_{s i}^{\mathrm{O}}-3 \times \sigma_{s i}^{\mathrm{O}}$. Também mostramos a curva normal associada a distribuição normal do custo do objeto, indicando a probabilidade de determinado custo ser sorteado (quanto mais distante do eixo $y$, maior a probabilidade do custo ser sorteado).

A Figura 4.6 mostra o logaritmo do tempo gasto, em segundos, na fase de treinamento para as combinações de valores de $\sigma$ e $\delta_{\text {ini }}$. Como podemos observar, agora temos uma variação bem grande 
entre o tempo gasto para diferentes valores de $\sigma$ e $\delta_{\text {ini }}$, sendo a combinação $\sigma=0,1$ e $\delta_{\text {ini }}=0,10$ a mais rápida (gastando cerca de 35 segundos) e a combinação $\sigma=0,8$ e $\delta_{\text {ini }}=0,30$ a mais demorada (gastando pouco mais de 1 hora e 30 minutos). Quando $\delta_{\text {ini }}=0,10$, independente do valor de $\sigma$, temos o menor tempo médio para o treinamento. O mesmo acontece para $\sigma=0,5$, independente do valor de $\delta_{\text {ini. }}$.

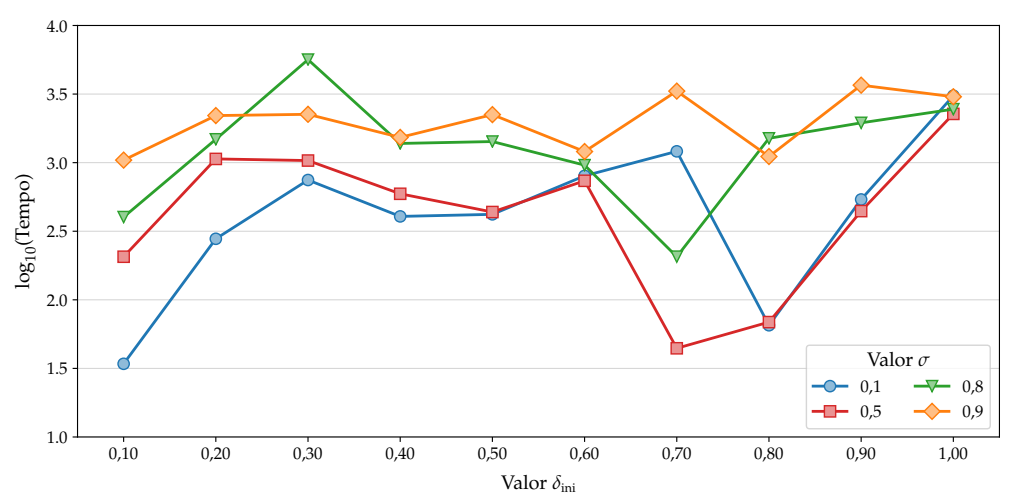

Figura 4.6: Tempo gasto, em escala logarítmica, na fase de treinamento para a PDA no Conjunto 2 para cada combinação de valor de $\delta_{\text {ini }}$ e $\sigma$.

Vamos agora analisar o resultado da fase de execução para o Conjunto 2 de problemas testes. O gráfico da Figura 4.7 resume o resultado obtido para cada combinação de valores de $\sigma$ e $\delta_{\text {ini }}$. Observe que agora há uma maior variação na solução obtida pela PDA em relação ao método guloso. Para esse conjunto, quatorze versões da PDA obtiveram ao menos uma solução pior que a solução encontrada pelo método guloso. São essas, $\operatorname{PDA}\left(\sigma=0,1 ; \delta_{\text {ini }}=0,10\right), \operatorname{PDA}\left(\sigma=0,1 ; \delta_{\text {ini }}=0,20\right)$, $\operatorname{PDA}\left(\sigma=0,1 ; \delta_{\text {ini }}=0,30\right), \operatorname{PDA}\left(\sigma=0,1 ; \delta_{\text {ini }}=0,40\right), \operatorname{PDA}\left(\sigma=0,1 ; \delta_{\text {ini }}=0,50\right), \operatorname{PDA}(\sigma=$ 0,$\left.1 ; \delta_{\text {ini }}=0,70\right), \operatorname{PDA}\left(\sigma=0,5 ; \delta_{\text {ini }}=0,10\right), \operatorname{PDA}\left(\sigma=0,8 ; \delta_{\text {ini }}=0,20\right), \operatorname{PDA}\left(\sigma=0,8 ; \delta_{\text {ini }}=0,30\right)$, $\operatorname{PDA}\left(\sigma=0,8 ; \delta_{\text {ini }}=0,40\right), \operatorname{PDA}\left(\sigma=0,8 ; \delta_{\text {ini }}=0,50\right), \operatorname{PDA}\left(\sigma=0,9 ; \delta_{\text {ini }}=0,20\right), \operatorname{PDA}(\sigma=$ 0,$\left.9 ; \delta_{\text {ini }}=0,30\right)$ e $\operatorname{PDA}\left(\sigma=0,9 ; \delta_{\text {ini }}=0,40\right)$. Por outro lado, as melhores versões da PDA foram: $\operatorname{PDA}\left(\sigma=0,5 ; \delta_{\text {ini }}=0,70\right), \operatorname{PDA}\left(\sigma=0,5 ; \delta_{\text {ini }}=0,80\right), \operatorname{PDA}\left(\sigma=0,5 ; \delta_{\text {ini }}=1,00\right), \operatorname{PDA}(\sigma=$ 0,$\left.8 ; \delta_{\text {ini }}=0,60\right), \operatorname{PDA}\left(\sigma=0,8 ; \delta_{\text {ini }}=0,70\right), \operatorname{PDA}\left(\sigma=0,8 ; \delta_{\text {ini }}=0,80\right), \operatorname{PDA}\left(\sigma=0,8 ; \delta_{\text {ini }}=1,00\right)$, $\operatorname{PDA}\left(\sigma=0,9 ; \delta_{\text {ini }}=0,80\right)$. Nessas versões, a solução encontrada pela PDA é, no mínimo, cerca de $30 \%$ melhor que a solução encontrada pelo método guloso. Perceba também que a maioria absoluta das soluções, encontradas por essas versões da PDA, são mais de $40 \%$ melhores que as soluções encontradas pelo método guloso. 


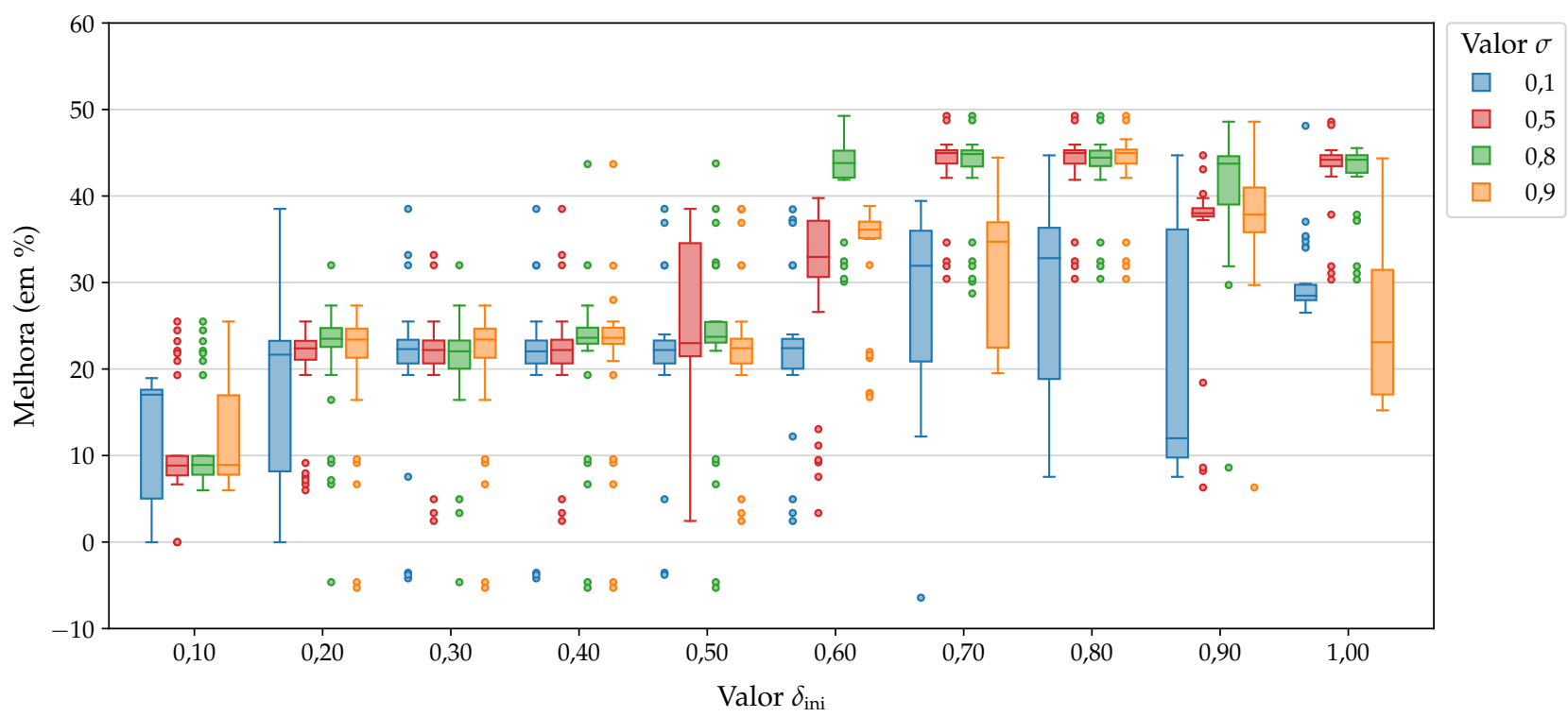

Figura 4.7: Resumo dos resultados encontrados pela PDA em comparação com o método guloso para o Conjunto 2.

O gráfico da Figura 4.8 apresenta a melhora média da PDA para diferentes valores de $\sigma$ e $\delta_{\text {ini }}$, considerando o Conjunto 2. Para esse conjunto, a $\operatorname{PDA}\left(\sigma=0,5 ; \delta_{\text {ini }}=0,10\right)$ foi a versão da PDA que obteve a pior média $(10,94 \%)$. Por outro lado, a $\operatorname{PDA}\left(\sigma=0,9 ; \delta_{\text {ini }}=0,80\right)$ foi a versão que se saiu melhor, conseguindo soluções, em média, $43,41 \%$ melhores que as soluções encontradas pelo método guloso. Com $\sigma=0,8$, independente do valor de $\delta_{\text {ini }}$, a PDA conseguiu melhores resultados. O mesmo acontece quando $\delta_{\text {ini }}=0,80$, independente do valor de $\sigma$. Para completar a análise das diferentes versões da PDA, a Tabela 4.3 mostra o número de instâncias em que a PDA obtém resultados melhores que o método guloso. Note que o maior número de instâncias em que o guloso consegue soluções melhores que a PDA é quando $\sigma=0,1$ e $\delta_{\text {ini }}=0,10$, onde a PDA não consegue soluções melhores em três instâncias. Além disso, também podemos notar que quando $\delta_{\text {ini }} \in\{0,60 ; 0,80 ; 0,90 ; 1,00\}$, independente do valor de $\sigma$, a PDA encontra soluções melhores que o guloso nas 30 instâncias testadas.

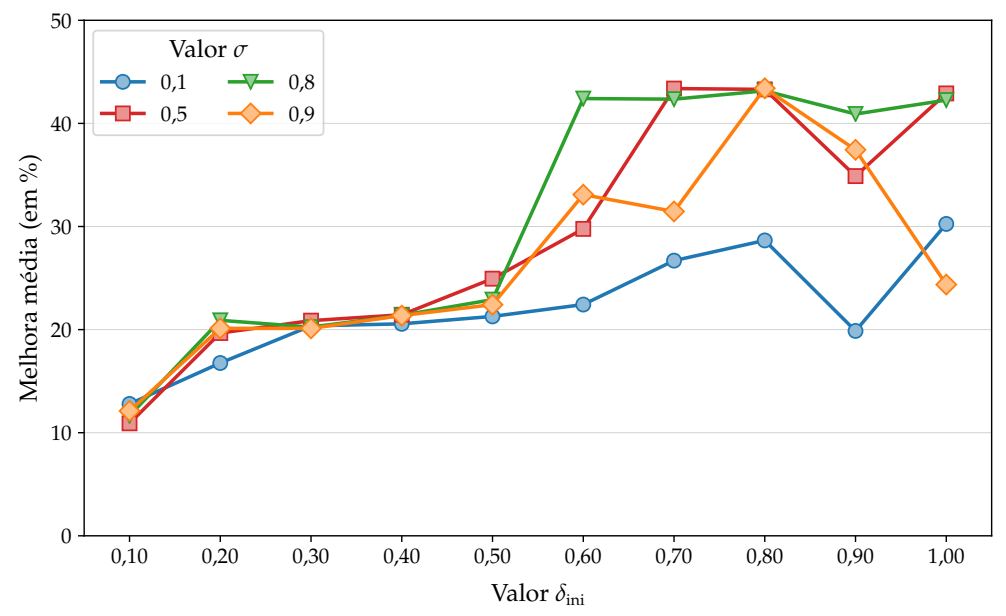

Figura 4.8: Melhora média (em porcentagem) para cada combinação de $\delta_{\text {ini }}$ e $\sigma$ para a PDA em relação ao método guloso, considerando o Conjunto 2 . 
Tabela 4.3: Número de soluções encontradas pela PDA que são melhores que as obtidas pelo método guloso no Conjunto 2.

\begin{tabular}{rrrrrrrrrrrrr}
\hline$\sigma$ & \multicolumn{10}{c}{$\delta_{\text {ini }}$} & \multirow{10}{*}{ Média } \\
\cline { 2 - 9 } & 0,10 & 0,20 & 0,30 & 0,40 & 0,50 & 0,60 & 0,70 & 0,80 & 0,90 & 1,00 & \\
\hline 0,1 & 27 & 28 & 27 & 27 & 28 & 30 & 29 & 30 & 30 & 30 & 28,60 \\
0,5 & 28 & 30 & 30 & 30 & 30 & 30 & 30 & 30 & 30 & 30 & 29,80 \\
0,8 & 30 & 29 & 29 & 28 & 28 & 30 & 30 & 30 & 30 & 30 & 29,40 \\
0,9 & 30 & 28 & 28 & 28 & 30 & 30 & 30 & 30 & 30 & 30 & 29,40 \\
\hline Média & 28,75 & 28,75 & 28,50 & 28,25 & 29,00 & 30,00 & 29,75 & 30,00 & 30,00 & 30,00 & 29,30 \\
\hline
\end{tabular}

O Conjunto 3 de problemas testes possui 8 períodos, dois tipos de objetos e três tipos de itens. Nesse conjunto, assim como no Conjunto 2, há uma variação do preço médio dos objetos ao longo do horizonte de planejamento, como mostra a Figura 4.9. Apesar da quantidade média de cada tipo de item ser pequena (Figura 4.10a) a soma dessa quantidade (Figura 4.10b) pode ser considerada média ou grande para ser resolvida pelo solver do modelo. Como nos três últimos períodos o preço médio dos objetos é maior que nos períodos anteriores e a média da quantidade dos itens diminui, é esperado que a PDA selecione objetos maiores e mais baratos nos períodos anteriores, de forma que a quantidade das sobras disponíveis, nos últimos períodos, faça com que o número de objetos necessários seja o menor possível.

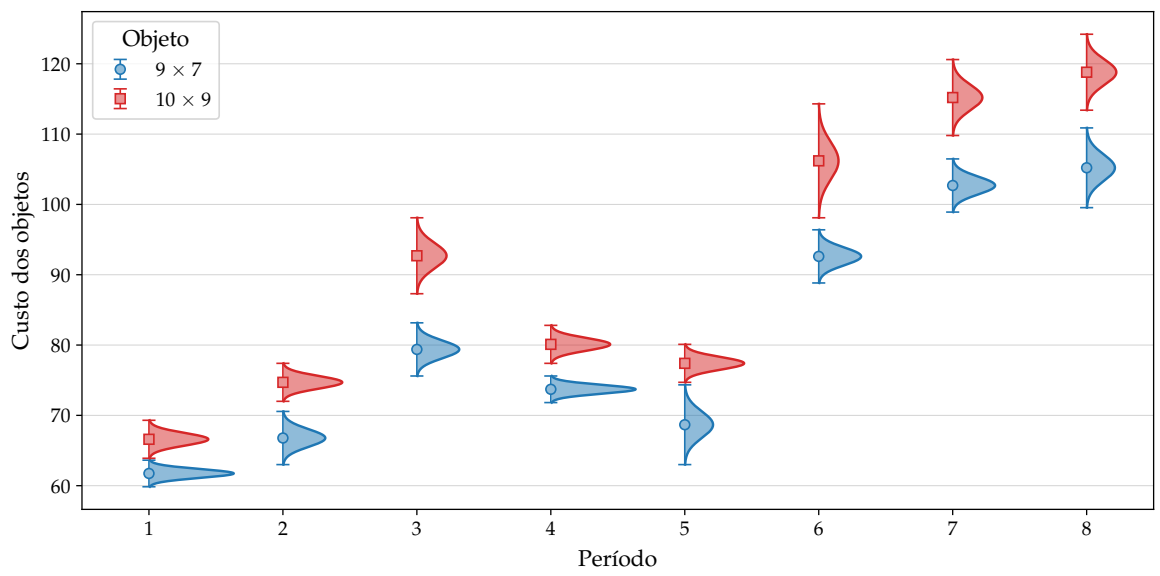

Figura 4.9: Custo médio dos objetos do Conjunto 3 em cada período.

O tempo gasto na fase de treinamento da PDA, para diferentes valores de $\sigma$ e $\delta_{\text {ini }}$, é mostrado no gráfico da Figura 4.11. Como esperado, quanto maior o valor de $\sigma$ e $\delta_{\text {ini }}$ maior o tempo gasto. Vale destacar que quando $\sigma=0,1$ e $\delta_{\text {ini }}=0,10$ o tempo necessário foi de menos de 5 minutos, enquanto para $\sigma=0,9$ e $\delta_{\text {ini }}=0,90$ o tempo gasto foi de mais de 16 horas. 


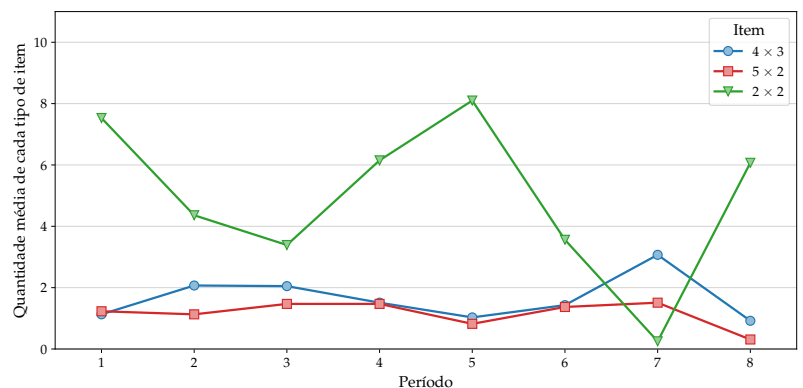

(a)

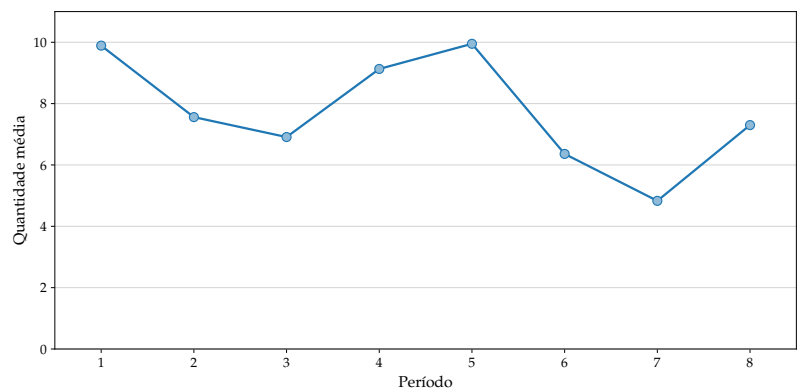

(b)

Figura 4.10: Quantidade média de cada tipo de item e quantidade média total dos itens do Conjunto 3.

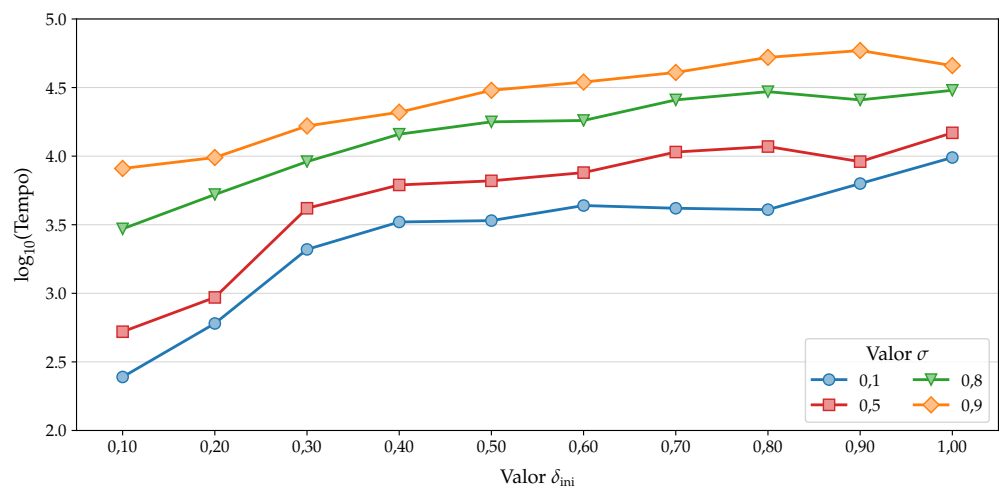

Figura 4.11: Tempo gasto, em escala logarítmica, na fase de treinamento para a PDA no Conjunto 3 para cada combinação de valor de $\delta_{\text {ini }}$ e $\sigma$.

Após a fase de treinamento, como feito nos outros conjuntos, realizamos a fase de execução. $\mathrm{O}$ gráfico da Figura 4.12 mostra os resultados resumidos para os diferentes valores de $\delta_{\text {ini }}$ e $\sigma$. Como podemos notar, quando $\sigma=0,1$ ou 0,5 temos uma maior variabilidade das soluções para a maioria dos valores de $\delta_{\text {ini }}$, mas para $\sigma=0,8$ ou 0,9 a variabilidade é bem menor, indicando que para essa configuração a PDA se comporta de forma mais consistente, encontrando quase sempre soluções com melhoras similares. Com a ajuda do gráfico da Figura 4.13, que apresenta a melhora média, é fácil notar que a melhora média aumenta com o crescimento de $\delta_{\text {ini }}$ e $\sigma$. Observe que a configuração $\sigma=0,1$ e $\delta_{\text {ini }}=0,9$ foi a que obteve a melhor melhora média (cerca de 27\%). Por outro lado, quando $\sigma=0,1$ e $\delta_{\text {ini }}=0,10$ a PDA não consegue encontrar soluções boas (melhora média de $-0,01 \%$ ), sendo ligeiramente pior que o método guloso. Isso porque, com essa configuração, a PDA fará poucas iterações até convergir e, $\operatorname{com} \delta_{\text {ini }}=0,10$, o objeto maior $(10 \times 9)$ terá poucas chances de ser selecionado durante as iterações da PDA. Perceba que para esse valor de $\delta_{\text {ini }}$, aumentando o número de iterações da PDA, a qualidade das soluções melhora, chegando a ser mais de $10 \%$, em média, melhor que o guloso. Com $\delta_{\text {ini }} \in\{0,40 ; 0,50 ; 0,60 ; 0,70 ; 0,80 ; 0,90 ; 1,00\}$, independente do valor de $\sigma$, a melhora média é sempre maior que $20 \%$ e não temos mais soluções piores que o guloso, indicando que a PDA encontrou uma solução melhor que o guloso em cada uma das 30 instâncias testadas. 


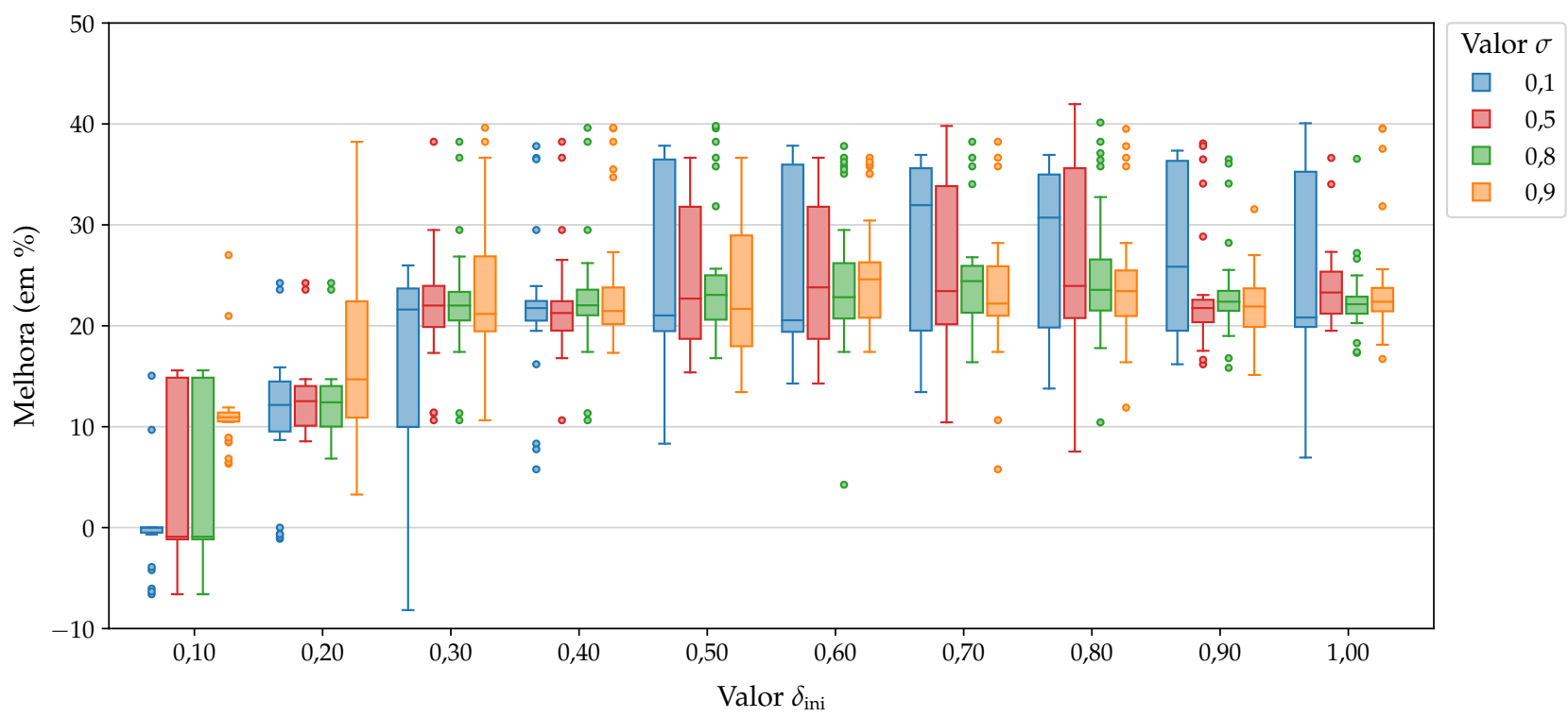

Figura 4.12: Resumo dos resultados encontrados pela PDA em comparação com o método guloso para o Conjunto 3.

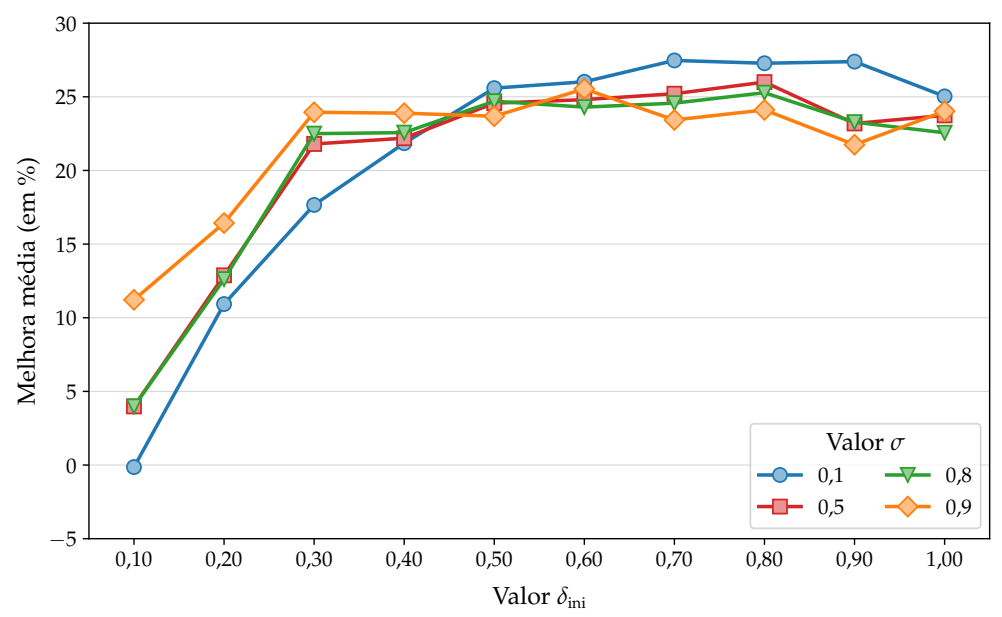

Figura 4.13: Melhora média (em porcentagem) para cada combinação de $\delta_{\text {ini }}$ e $\sigma$ para a PDA em relação ao método guloso, considerando o Conjunto 3.

O Conjunto 4 de problemas testes possui 8 períodos, cada um com dois tipos de objetos e podendo ter até 5 tipos de itens. O gráfico da Figura 4.14 mostra as variações dos preços dos dois objetos ao longo do horizonte de planejamento. Note que a diferença entre os dois objetos é relativamente pequena, de modo que os dois objetos têm chances de serem selecionados pela PDA, dependendo dos valores das estimativas da proporção de utilização das sobras dos objetos. A maior diferença pode ocorrer no período 5 , onde o objeto $15 \times 10$ pode custar 147,00 unidades monetárias (valor mínimo) enquanto o objeto $20 \times 10$ pode custar 204,00 (valor máximo). Nesse caso, a diferença é de 57,00 e, dependendo dos valores das estimativas da proporção de utilização das sobras dos objetos, o objeto $20 \times 10$ pode ter um custo "artificial" superior ao do outro objeto e não ter a chance de ser selecionado. Há também uma variação na quantidade média de cada item (veja os gráficos da Figura 4.15) ao longo dos oito períodos. Essa variação segue, de certa forma, a variação do preço dos 
objetos: quando a quantidade média dos itens solicitados é maior, os objetos são mais caros. O que é natural em aplicações reais, devido às variações sazonais.

Note que, nesse conjunto, as sobras têm maior probabilidade de possuírem largura e altura iguais a 2, uma vez que o item do catálogo possui essa dimensão e que, em quase todos os períodos, os itens $2 \times 2$ e $3 \times 2$ são os que possuem a maior quantidade média. Isso é um fator que pode ajudar a PDA, já que teremos maior chance de usarmos todos os objetos originados de sobras anteriores, sem que seja necessário a compra de objetos novos, de forma que a proporção de utilização desses objetos seja quase total.

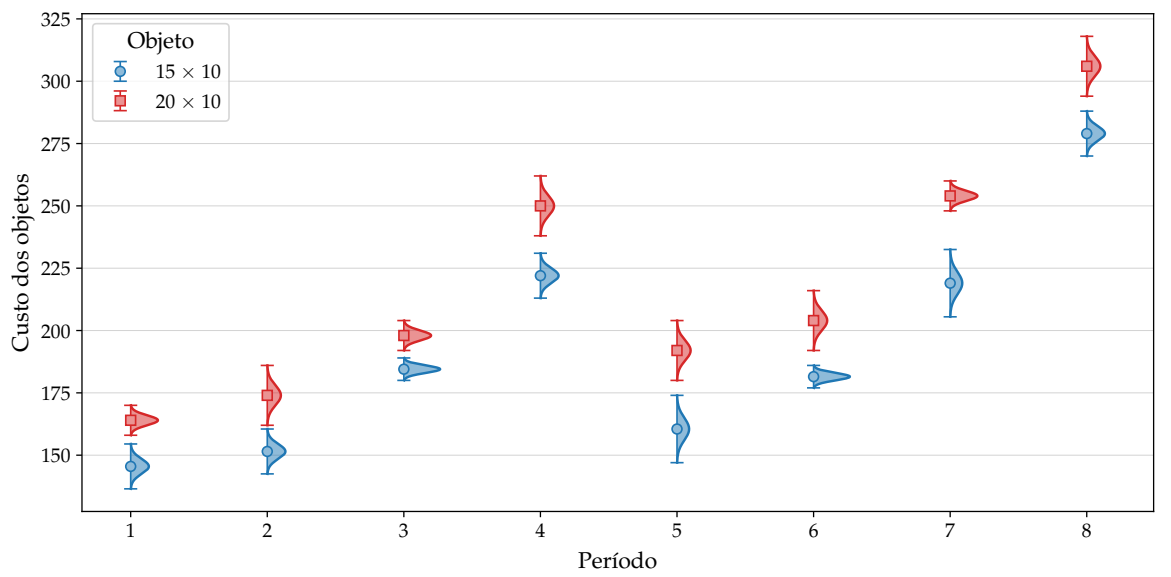

Figura 4.14: Custo médio dos objetos do Conjunto 4 em cada período.

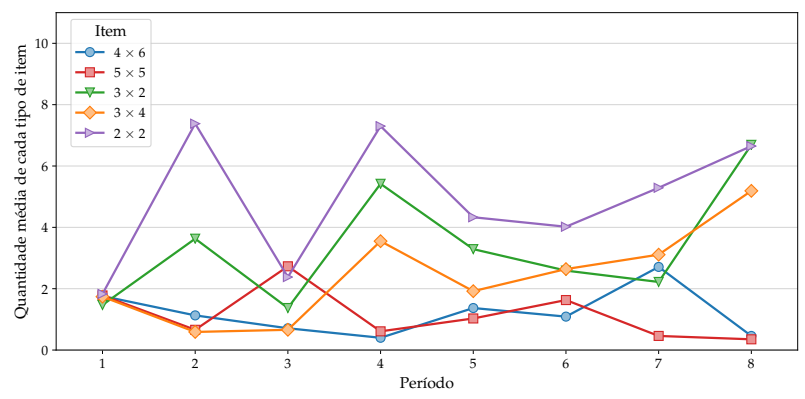

(a)

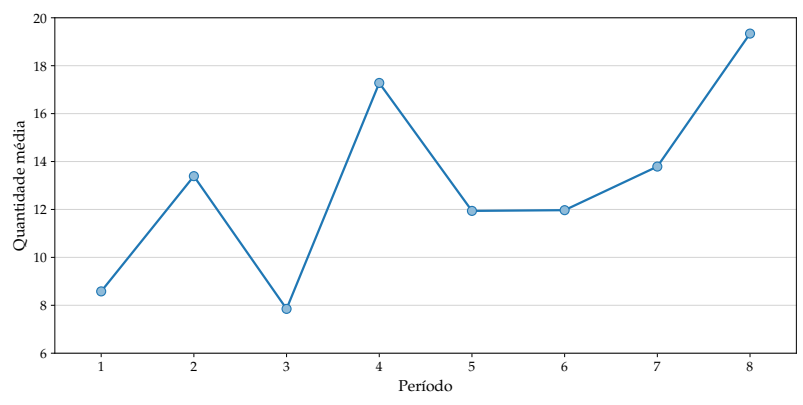

(b)

Figura 4.15: Quantidade média de cada tipo de item e quantidade média total dos itens do Conjunto 4.

No gráfico da Figura 4.16 mostramos o tempo gasto, em escala logarítmica, na fase de treinamento da PDA para o Conjunto 4, considerando diferentes valores de $\delta_{\text {ini }}$ e $\sigma$. Assim como nos outros conjuntos de problemas testes, o tempo gasto aumenta conforme aumentamos o valor de $\sigma$. É interessante notar que para cada valor de $\sigma$ o tempo se mantém muito próximo para os diferentes valores de $\delta_{\text {ini. }}$ O tempo de treinamento nesse conjunto foi alto porque na maioria das iterações da PDA, cada resolução do modelo $\tilde{\mathcal{M}}_{s, s}^{\mathrm{C}_{\max }}$ pelo CPLEX, atingiu o tempo limite de 10 minutos, fazendo com que o tempo médio para cada $\sigma$ varie de, aproximadamente, 3 horas (quando $\sigma=0,1$ ) a quase 48 horas (quando $\sigma=0,9$ ). 


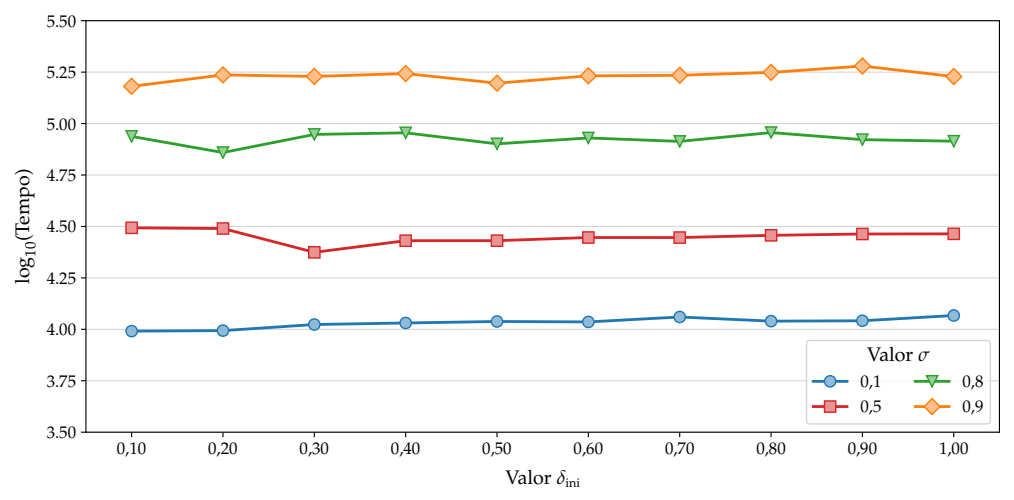

Figura 4.16: Tempo gasto, em escala logarítmica, na fase de treinamento para a PDA no Conjunto 4 para cada combinação de valor de $\delta_{\text {ini }}$ e $\sigma$.

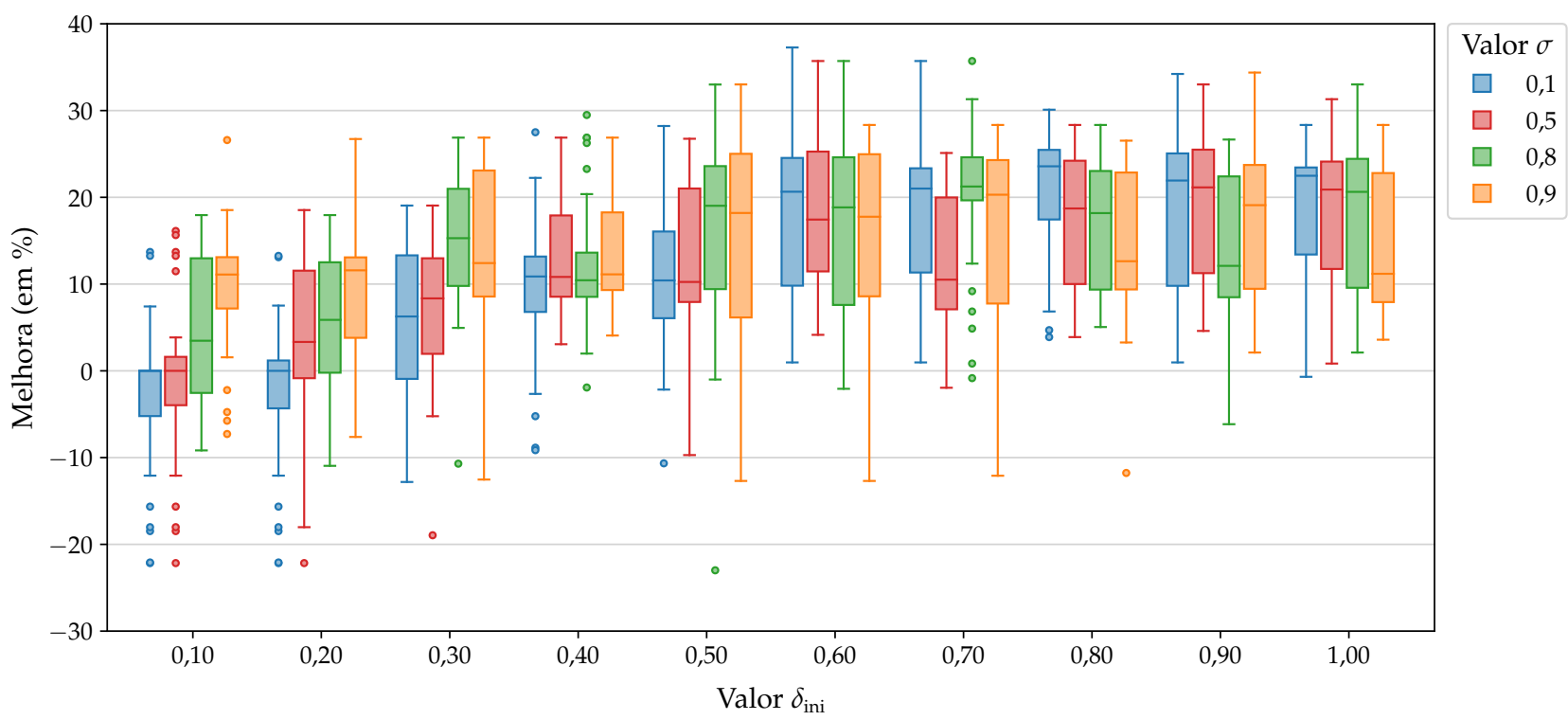

Figura 4.17: Resumo dos resultados encontrados pela PDA em comparação com o método guloso para o Conjunto 4.

O gráfico da Figura 4.17 apresenta o resumo dos resultados encontrados para as instâncias geradas a partir das características do Conjunto 4. Note que não há muita variação na melhora para as diferentes versões da PDA, mas quando $\delta_{\text {ini }}$ é igual a 0,10 ou 0,20 a maioria das soluções são, no máximo, $10 \%$ melhores que as soluções do método guloso. Quando $\delta_{\text {ini }}=0,10$, para os diferentes valores de $\sigma$, a média do número das melhores soluções é 18 (a menor quantidade), enquanto para $\delta_{\text {ini }} \in\{0,80 ; 0,90 ; 1,00\}$ essa mesma média foi de 29,75 (para cada uma dessas configurações a solução de apenas uma instância não foi melhor que a solução encontrada pelo guloso). No gráfico da Figura 4.18 temos a melhora média. Pelo gráfico, podemos notar novamente que, na maioria dos casos, a melhora média cresce à medida que aumentamos o valor de $\delta_{\text {ini }}$ e $\sigma$. Para $\delta_{\text {ini }}=0,10$ e $\sigma \in\{0,1 ; 0,5\}$ temos os piores resultados médios, indicando que a PDA encontrou muitas soluções piores que as encontradas pelo método guloso. Por outro lado, quando $\delta_{\text {ini }}=0,80$ e $\sigma=0,1$ temos mais de $20 \%$ de melhora média. 


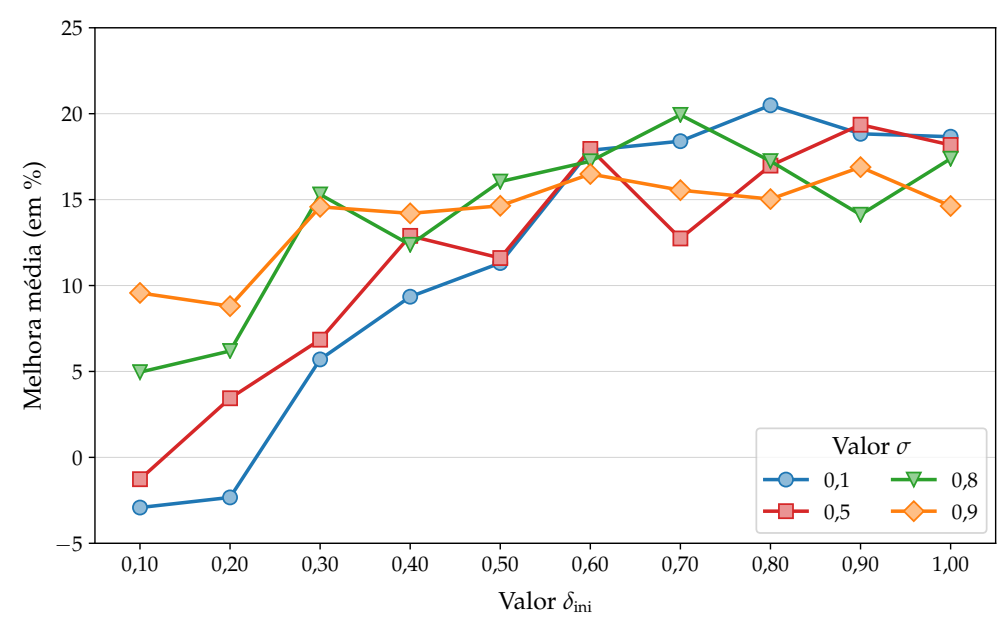

Figura 4.18: Melhora média (em porcentagem) para cada combinação de $\delta_{\text {ini }}$ e $\sigma$ para a PDA em relação ao método guloso, considerando o Conjunto 4 .

O Conjunto 5 de problemas testes possui informações de 4 períodos, sendo que em cada período temos 3 tipos de objetos que estão disponíveis e 5 tipos de itens que podem ser solicitados em alguma ordem de trabalho. O gráfico da Figura 4.19 mostra a variação do custo de cada objeto ao longo do horizonte de planejamento. Novamente, a diferença do preço dos objetos é relativamente pequena, o que faz com que todos os objetos tenham chances de serem selecionados pela PDA, dependendo dos valores das estimativas de proporção de utilização das sobras dos objetos. Como no último período temos um preço médio dos objetos elevado, em comparação com o preço dos outros períodos, é desejável que nesse período não seja necessário comprar nenhum objeto. Assim, é esperado que a PDA compre objetos maiores nos períodos anteriores, de forma que as sobras disponíveis no quarto período sejam suficientes para atender a ordem de trabalho dos itens desse período. Os gráficos da Figura 4.20 mostram a quantidade média de cada tipo de item (Figura 4.20a) e a soma dessa quantidade (Figura 4.20b), observe que no quarto período temos a menor quantidade média de itens, em comparação com os outros períodos, e os itens com média maior são objetos com menor área. Dessa forma, aumentamos a chance de não precisarmos comprar objetos, dependendo da quantidade e dimensões das sobras disponíveis, nesse período (cujo preço dos objetos é mais elevado).

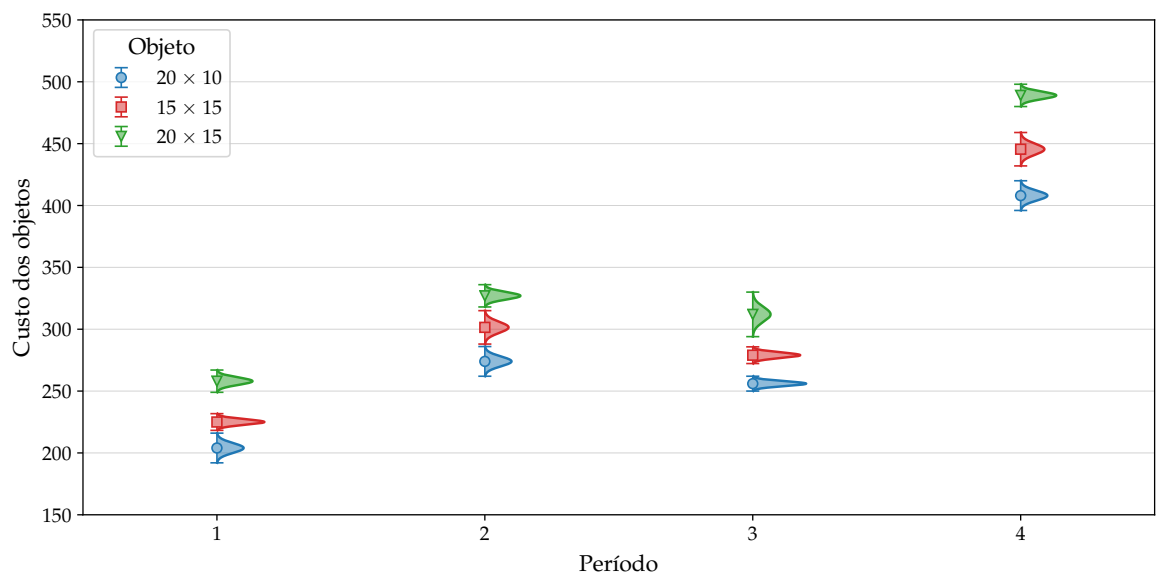

Figura 4.19: Custo médio dos objetos do Conjunto 5 em cada período. 


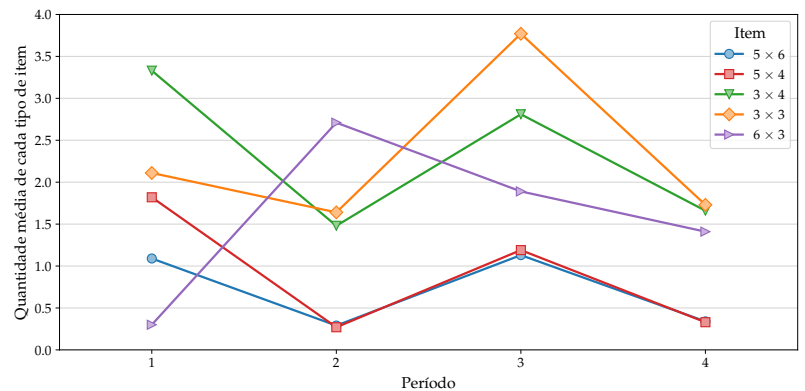

(a)

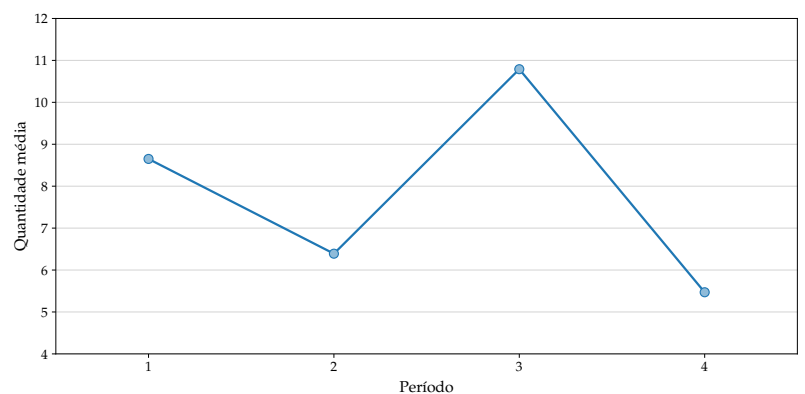

(b)

Figura 4.20: Quantidade média de cada tipo de item e quantidade média total dos itens do Conjunto 5.

O gráfico da Figura 4.21 apresenta os resultados, resumidos, da resolução das 30 instâncias (geradas seguindo as características do Conjunto 5) pela PDA. Como podemos ver, nesse conjunto temos uma maior variação de melhora para os diferentes valores de $\delta_{\text {ini }}$ e $\sigma$. Além disso, nenhuma versão da PDA conseguiu obter sempre soluções melhores que as soluções encontradas pelo método guloso, ou seja, todas as versões apresentam pelo menos uma solução cuja melhora é abaixo de $0 \%$. Essa maior variação pode ocorrer devido às características do conjunto: veja que os itens $5 \times 6$ e $5 \times 4$ têm uma probabilidade muito pequena de estarem na ordem de trabalho do último período, ou seja, a PDA "aprende" que não vale a pena investir em objetos maiores e, possivelmente, mais caros para atender a ordem de trabalho desses itens, que dificilmente serão solicitados. Entretanto, em instâncias que eles surgem, a qualidade da solução tende a ser baixa ou até pior que uma solução gulosa. Outro fator que também pode influenciar a qualidade das soluções é o tempo limite do CPLEX.

Observe que na PDA com $\delta_{\text {ini }}=0,20$, qualquer que seja o valor de $\sigma$, a maioria das soluções são praticamente iguais às soluções encontradas pelo guloso (uma vez que a melhora é cerca de $0 \%$ ). Isso também ocorre quando $\delta_{\text {ini }}=0,10$ e $\sigma=0,8$ ou 0,9 ; quando $\delta_{\text {ini }}=0,30$ e $\sigma=0,1$ ou 0,5 ; e quando $\delta_{\text {ini }}=0,60$ e $\sigma=0,1$ ou 0,9 . A PDA com $\sigma=0,8$ e $\delta_{\text {ini }}=0,40$ e a PDA com $\sigma=0,9$ e $\delta_{\text {ini }}=0,90$ são as versões que obtiveram o maior número de soluções de maior custo em relação ao método guloso, ou seja, encontraram a maior quantidade de soluções piores que o guloso. Como mostra a Figura 4.22, essas versões da PDA são as que obtiveram a menor melhora média, sendo suas soluções, em média, $5 \%$ piores que as soluções do guloso. Por outro lado, a PDA com $\sigma=0,5$ e $\delta_{\text {ini }}=0,70$ e a PDA com $\sigma=0,5$ e $\delta_{\text {ini }}=1,00$ são as duas versões que obtiveram o maior número de melhores soluções, com melhora próxima de $40 \%$. Nessas duas versões, a média da melhora foi de aproximadamente $35 \%$ (veja a Figura 4.22). A PDA com $\sigma=0,9$, para os diferentes valores de $\delta_{\text {ini, }}$, obteve soluções 6,5\%, em média, melhores que as soluções do guloso, já para a PDA com $\sigma=0,5$ essa média é de pouco mais de $16 \%$. Fazendo essa análise pelo valor de $\delta_{\text {ini }}$, quando $\delta_{\text {ini }}=0,20$ a PDA obtém soluções com menor média $(2 \%)$ e quando $\delta_{\text {ini }}=0,70$ a PDA encontra soluções com a maior média (23\%). 


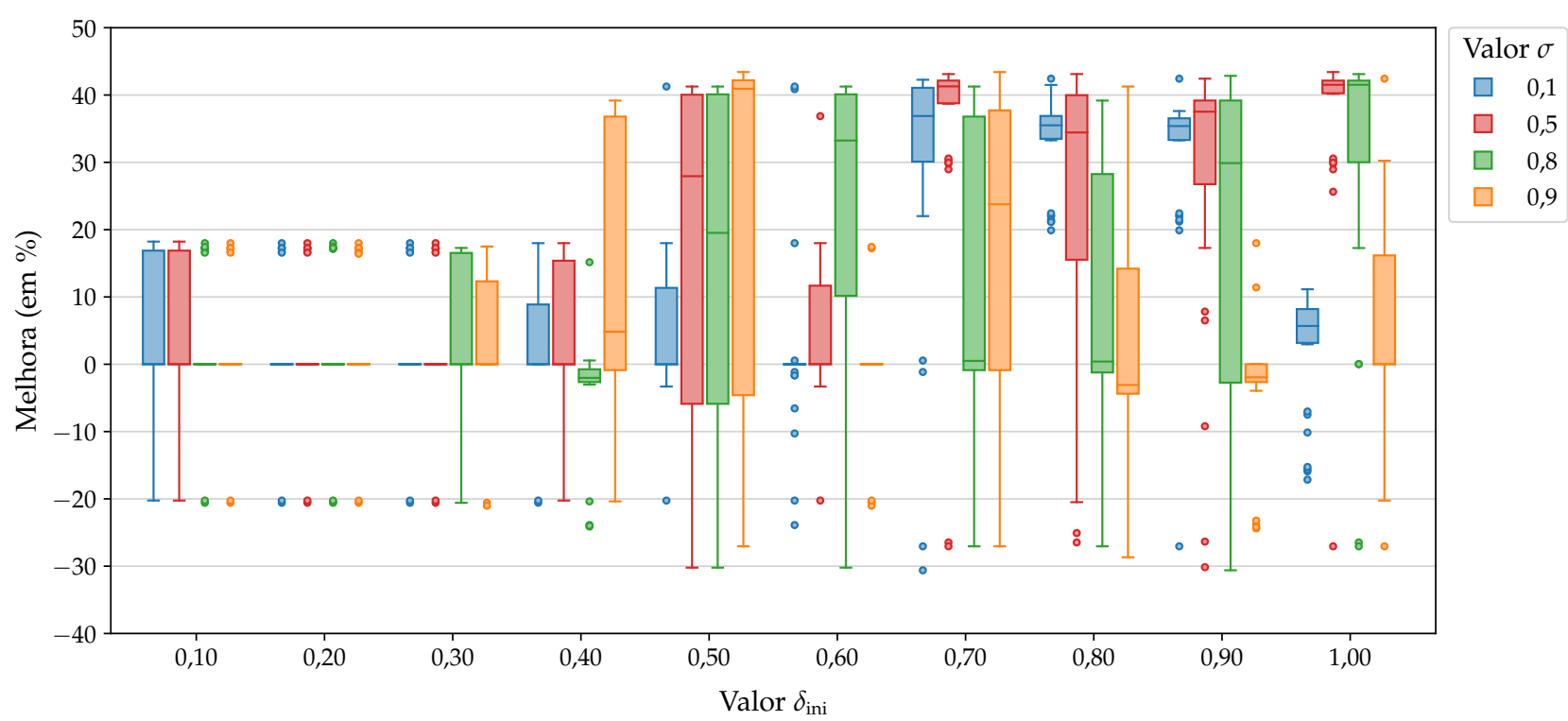

Figura 4.21: Resumo dos resultados encontrados pela PDA em comparação com o método guloso para o Conjunto 5.

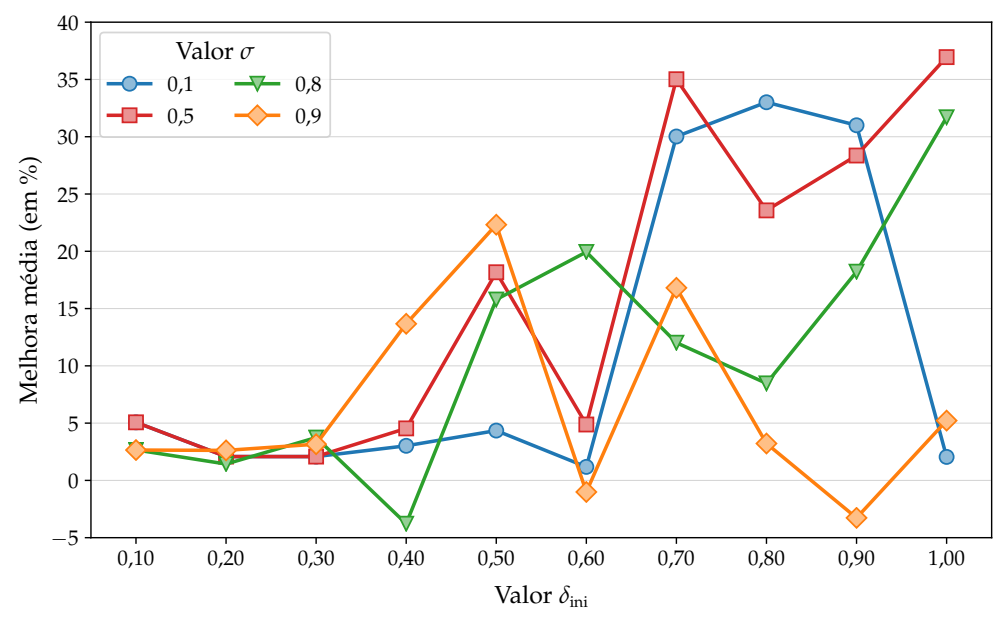

Figura 4.22: Melhora média (em porcentagem) para cada combinação de $\delta_{\text {ini }}$ e $\sigma$ para a PDA em relação ao método guloso, considerando o Conjunto 5 .

O Conjunto 6 de problemas testes possui informações de 4 períodos, sendo que em cada período temos 3 tipos de objetos que estão disponíveis e 5 tipos de itens. Esse conjunto é muito similar ao Conjunto 5, a diferença está na média e desvio padrão, no segundo e quarto período dos itens $5 \times 6 \mathrm{e}$ $5 \times 4$, mantendo assim todas as outras informações (inclusive a variação do preço dos objetos). Dessa forma, podemos ver como a PDA se comporta com o aumento da quantidade de itens grandes (em relação ao item do catálogo). Com isso, de certa forma, violamos a primeira suposição que fizemos para que a PDA consiga soluções, em média, de melhor qualidade que o método guloso.

O gráfico da Figura 4.23 resume os resultados das soluções das instâncias geradas e testadas pela PDA. Como esperado, a PDA não teve um resultado satisfatório, principalmente quando $\delta_{\text {ini }} \in$ $\{0,10 ; 0,20 ; 0,30\}$, para os diferentes valores $\sigma$, onde a maioria das soluções foram praticamente iguais às encontradas pelo método guloso. Para os outros valores de $\delta_{\text {ini }}$ a PDA conseguiu soluções 
um pouco melhores, mas ainda há uma variabilidade grande e muitas soluções piores que as gulosas, na maiorias das configurações. Além disso, a PDA, para os valores de $\delta_{\text {ini }}$ e $\sigma$ testados, não consegue encontrar para todas as 30 instâncias soluções melhores que o método guloso, ou seja, em pelo menos uma instância o método guloso encontra uma solução melhor que a PDA, em alguns casos a PDA encontra algumas soluções, quase, $60 \%$ piores que o método guloso. Em 8 versões a PDA encontrou ao menos uma solução com melhora acima de 40\%, mas, mesmo nessas versões, muitas soluções foram piores que as soluções gulosas.

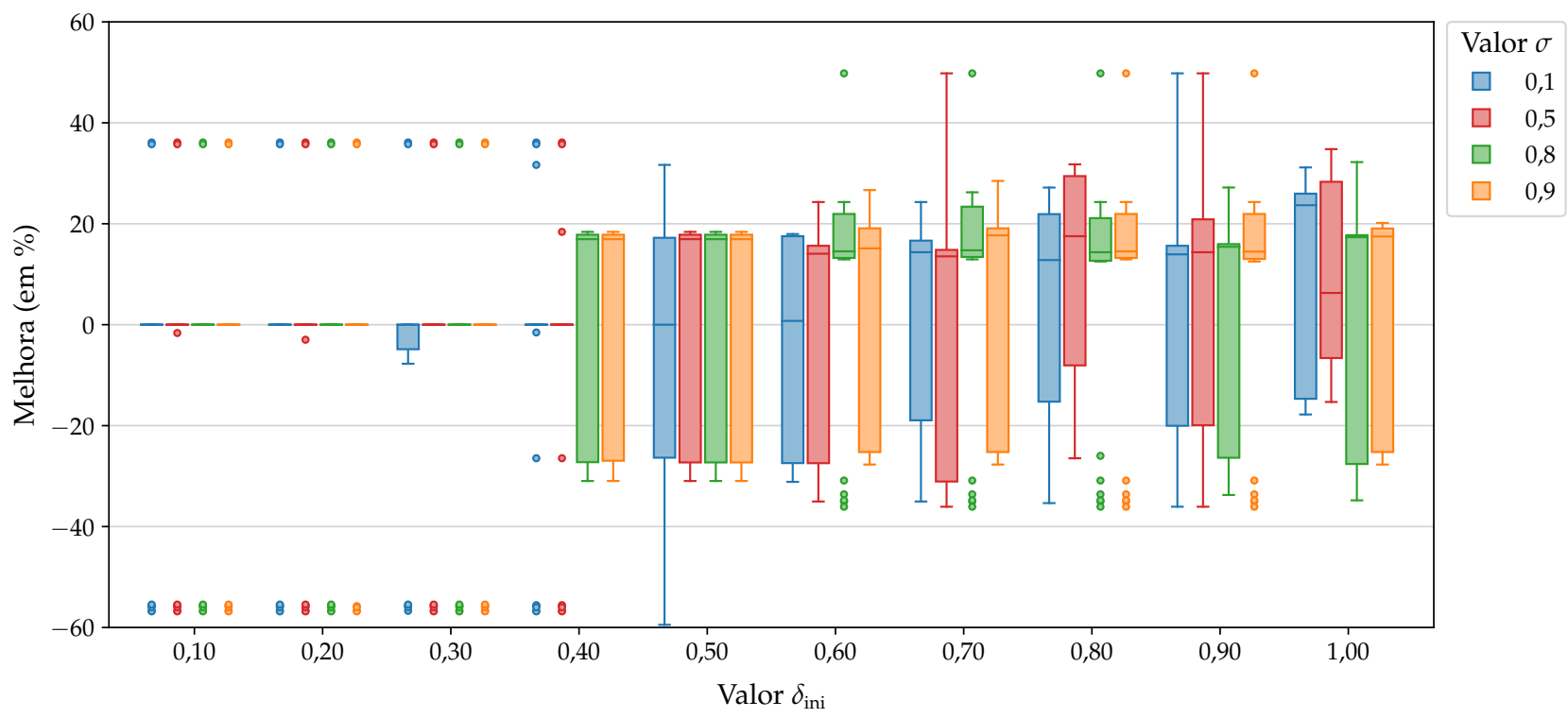

Figura 4.23: Resumo dos resultados, para o Conjunto 6, obtidos pela PDA em comparação com o método guloso.

Pelo gráfico da Figura 4.24 notamos que a melhora média para $\delta_{\text {ini }} \in\{0,10 ; 0,20 ; 0,30 ; 0,40 ; 0,50\}$, para os diferentes valores $\sigma$, é abaixo de zero. Apenas quatro versões da PDA encontraram soluções que, em média, sejam mais de $10 \%$ melhores que as soluções gulosas, entre essas quatro, a PDA com $\sigma=0,8$ e $\delta_{\text {ini }}=0,70$ foi a que encontrou a melhora média mais alta (próxima de $11 \%$ ).

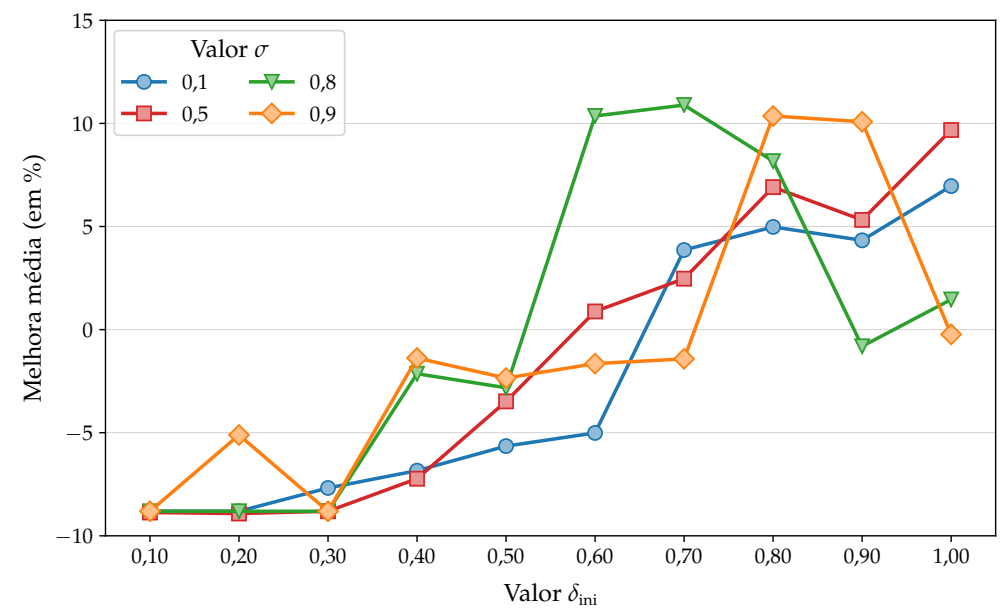

Figura 4.24: Melhora média (em porcentagem) para cada combinação de $\delta_{\text {ini }}$ e $\sigma$ para a PDA em relação ao método guloso, considerando o Conjunto 6. 
O último conjunto de problemas testes, Conjunto 7, também é similar ao Conjunto 5, mas nesse o custo por unidade de área dos objetos em cada período é muito próximo, de forma que os objetos maiores terão um custo mais elevado em comparação com os objetos menores. O gráfico da Figura 4.25 mostra o custo médio dos objetos em cada período. Perceba que a diferença do preço médio entre o objeto $20 \times 15$ (maior objeto) e o objeto $15 \times 15$ e entre o objeto $20 \times 15$ e o objeto $20 \times 10$ (menor objeto) é, quase sempre, maior que 70 unidades monetárias (no Conjunto 5 essa diferença era bem menor). Isso faz com que o objeto $20 \times 15$ tenha poucas chances de ser selecionado pela PDA, independente dos valores das estimativas da proporção de utilização das sobras dos objetos. Como o preço médio entre os objetos $15 \times 15$ e $20 \times 10$ é próximo, o objeto $15 \times 15$ terá mais chances de ser selecionado pela PDA que o objeto $20 \times 15$. Entretanto, o objeto $15 \times 15$ possui área cerca de $10 \%$ maior que a área do objeto $20 \times 10$, com isso as sobras desses objetos tendem a terem áreas similares, o que pode, como veremos, não ser muito vantajoso para a solução global desse conjunto de problemas testes. Vale destacar que para esse conjunto temos $q_{s j}^{\mathrm{O}}=1$, para $s=1, \ldots, 4, j=1, \ldots, m_{s}$, ou seja, em cada período temos apenas um objeto de cada tipo disponível para compra.

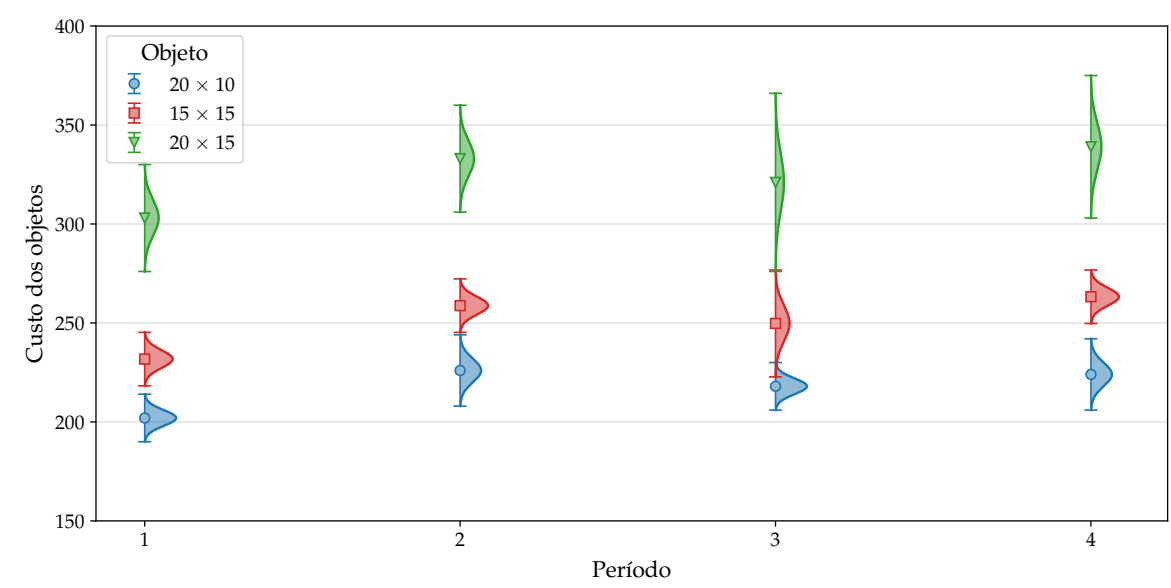

Figura 4.25: Custo médio dos objetos do Conjunto 7 em cada período.

O gráfico da Figura 4.26 apresenta a melhora encontrada pelas diferentes versões da PDA. Como esperado, as soluções encontradas pela PDA foram similares às obtidas pelo método guloso (melhora próxima de $0 \%$ ). Como o custo dos objetos é bem diferente, a variabilidade da melhora é pequena em relação aos outros conjuntos em que o custo dos objetos eram mais próximos. Apenas quando $\delta_{\text {ini }}=0,80$ e $\sigma=0,5$ a PDA encontra uma quantidade maior de soluções melhores que o guloso, tendo uma maior variabilidade. Perceba que apenas seis versões da PDA encontraram ao menos uma solução com mais de $10 \%$ de melhora e 11 versões encontraram ao menos uma solução que foi mais de $10 \%$ pior que o guloso. O gráfico da Figura 4.27 mostra a melhora média para as diferentes combinações de valores de $\delta_{\text {ini }}$ e $\sigma$ da PDA. Note que a grande maioria foi, em média, muito similar ou pior que o guloso. Apenas na PDA com $\delta_{\text {ini }}=0,80$ e $\sigma=0,5$ e na PDA com $\delta_{\text {ini }}=0,70$ e $\sigma=0,9$ as soluções foram melhores, em média, que as obtidas pelo método guloso. A PDA com $\delta_{\text {ini }}=1,00$ e $\sigma=0,1$ foi a versão que encontrou as piores soluções, sendo, em média, mais de $15 \%$ pior que o método guloso. 


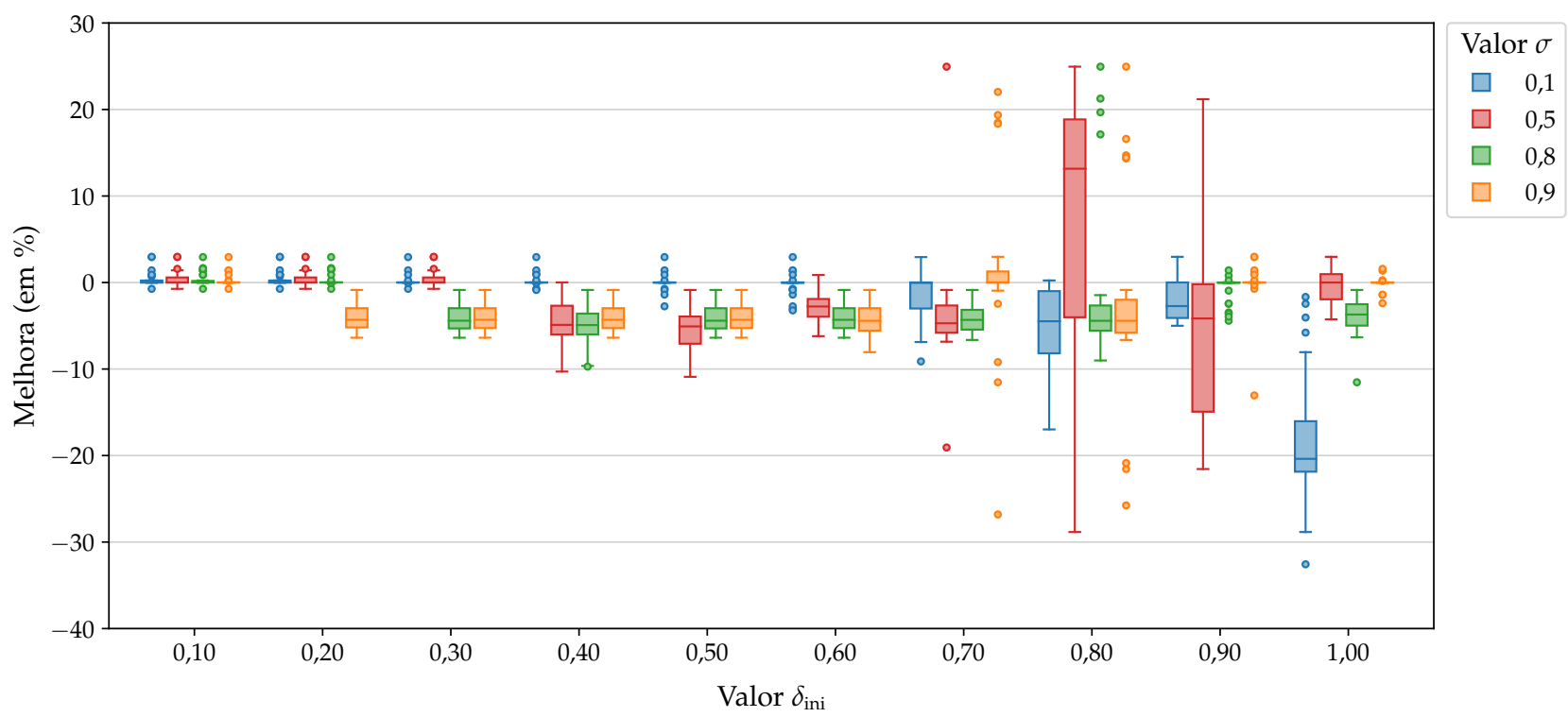

Figura 4.26: Resumo dos resultados encontrados pela PDA em comparação com o método guloso para o Conjunto 7.

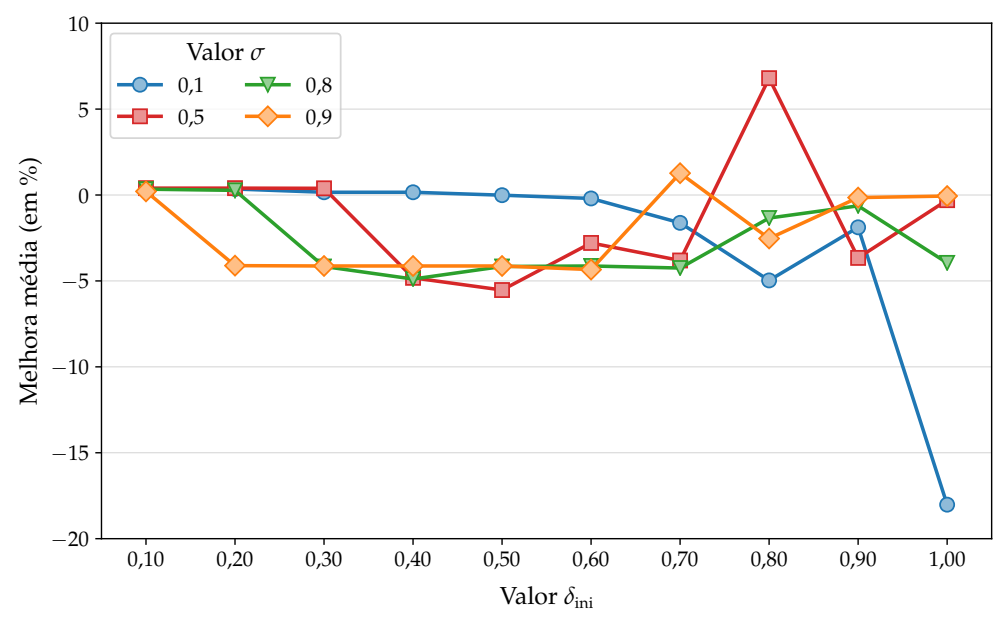

Figura 4.27: Melhora média (em porcentagem) para cada combinação de $\delta_{\text {ini }}$ e $\sigma$ para a PDA em relação ao método guloso, considerando o Conjunto 7 .

O fato da PDA encontrar soluções piores que o método guloso, está relacionado ao preço e tamanho dos objetos. Por exemplo, considere uma instância em que nos três primeiros períodos sempre é preciso comprar um objeto em cada período para atender a ordem de trabalho dos itens, independente das sobras disponíveis. Nos primeiros períodos a PDA provavelmente irá selecionar o objeto $15 \times 15$ (de maior tamanho que o objeto $20 \times 10$, mas, quase sempre, mais caro) e no último período o objeto $20 \times 10$ (já que as estimativas de proporção de utilização das sobras não são consideradas). Dessa forma, uma solução poderia ser: comprar três objetos do tipo $15 \times 15$ e um do tipo $20 \times 10$, cujo custo médio é de 964,25 unidades monetárias. Já o método guloso irá sempre escolher, em cada período, o objeto de menor custo que seja suficiente para atender a ordem de trabalho do período. Como $q_{s j}^{\mathrm{o}}=1$, para $s=1, \ldots, 4, j=1, \ldots, m_{s}$, o método guloso sempre selecionará o objeto $20 \times 10$ (exceto talvez no terceiro período), cujo custo médio é o mais barato. 
Assim, uma solução encontrada pelo método guloso poderia ser: comprar quatro objetos $20 \times 10$, cujo custo médio de 870 unidades monetárias. Tal solução é $10 \%$ mais barato que o custo encontrado pela solução da PDA.

Vamos agora analisar a PDA considerando os sete conjuntos de problemas testes. Os gráficos da Figura 4.28 apresentam a quantidade média de soluções em que a PDA (para diferentes valores de $\sigma$ e $\delta_{\text {ini }}$ ) foi melhor que o método guloso. Como em cada conjunto geramos 30 instâncias, quanto mais próximo de 30 melhor. A Figura 4.28a mostra o gráfico da quantidade média considerando apenas os conjuntos que atendem as propriedades para que a PDA tenha um resultado satisfatório, ou seja, os conjuntos de 1 a 5 . Como podemos notar, quando $\sigma \in\{0,1 ; 0,5 ; 0,8\}$ a quantidade média de soluções melhores tende a aumentar a medida que aumentamos o valor de $\delta_{\text {ini }}$. Em especial quando $\sigma \in\{0,1 ; 0,5\}$ esse aumento é ainda mais evidente. Quando $\sigma=0,9$ não há muita variação para os diferentes valores de $\delta_{\text {ini }}$, onde a maior quantidade média é 28 (para $\delta_{\text {ini }}=1,00$ ) e a menor é 25,8 (com $\left.\delta_{\text {ini }}=0,90\right)$. Para esses conjuntos, a PDA obtém a maior quantidade média quando $\sigma=0,1$ e $\delta_{\text {ini }}=0,80$ e a menor ocorre com $\sigma=0,1$ ou $\sigma=0,5$ e $\delta_{\text {ini }}=0,10$. Já o gráfico da Figura $4.28 \mathrm{~b}$ apresenta a quantidade média considerando os sete conjuntos testados, isto é, os conjuntos adequados para a PDA e os conjuntos em que a PDA tende a não apresentar bons resultados. É fácil notar que a quantidade média reduz (quando comparado ao gráfico da Figura 4.28a) para todos os valores de $\sigma$ e $\delta_{\text {ini, }}$, sendo um pouco abaixo de 16 quando $\sigma=0,5$ e $\delta_{\text {ini }}=0,10$ e próximo de 26 para $\sigma=0,1$ e $\delta_{\text {ini }}=0,90$.

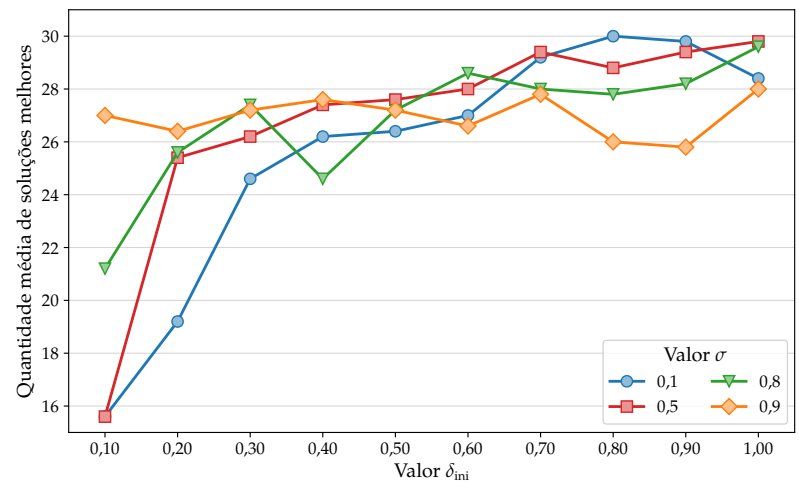

(a) Número médio de soluções melhores para os conjuntos 1 a 5

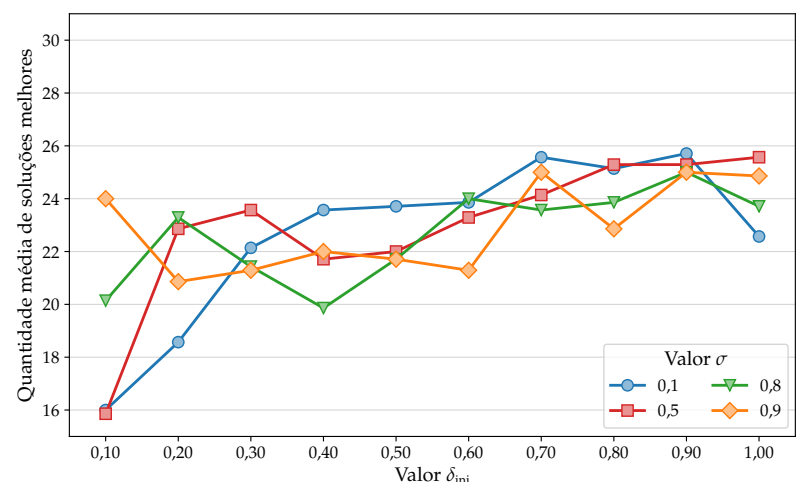

(b) Número médio de soluções melhores para os conjuntos 1 a 7

Figura 4.28: Quantidade média de soluções em que a PDA foi melhor que o método guloso considerando todas as instâncias dos sete conjuntos.

Os gráficos da Figura 4.29 exibem a quantidade média de soluções em que a PDA foi melhor que o método guloso para os diferentes valores de $\delta_{\text {ini }}$ (Figura 4.29a) e para os diferentes valores de $\sigma$ (Figura 4.29b). Observando o gráfico da Figura 4.29a, notamos que os melhores resultados em relação aos conjuntos favoráveis para a PDA aparecem quando $\delta_{\text {ini }} \in\{0,70 ; 0,80 ; 0,90 ; 1,00\}$, para os diferentes valores de $\sigma$, mas para os conjuntos 6 e 7, a PDA apresenta a maior quantidade média quando $\delta_{\text {ini }}=0,90$. Calculando essa mesma média para os sete conjuntos, a maior quantidade média também ocorre quando $\delta_{\text {ini }}=0,90$ e a menor com $\delta_{\text {ini }}=0,10$. Pelo gráfico da Figura 4.29b, quando consideramos os conjuntos 1 a 5, vemos que a quantidade média sofre pouca variação com a mudança 
no valor de $\sigma$. Para os conjuntos 6 e 7 a quantidade média é bem menor e há uma variação maior. Entretanto, para os sete conjuntos a quantidade média é sempre cerca de 23.

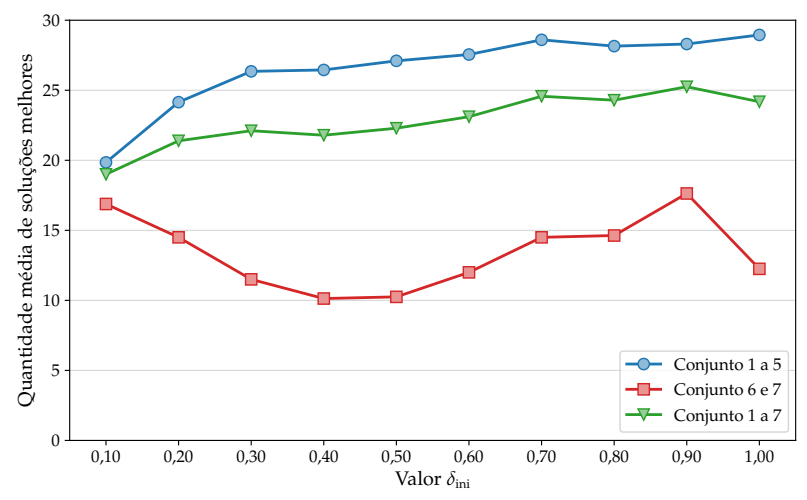

(a) Soluções melhores por $\delta_{\text {ini }}$

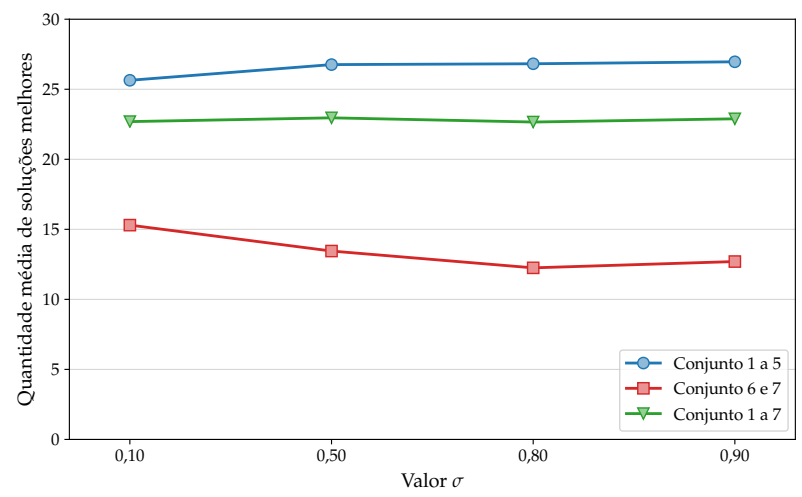

(b) Soluções melhores por $\delta_{\text {ini }}$

Figura 4.29: Quantidade média geral de soluções em que a PDA foi melhor que o método guloso.

A quantidade média de soluções da PDA melhores que o método guloso não diz muita coisa em relação à qualidade das soluções. Podemos, então, calcular a melhora média dos conjuntos testados para os diferente valores de $\sigma$ e $\delta_{\text {ini }}$. Analisando a média apenas para os conjuntos propícios para a PDA (gráfico da Figura 4.30a), vemos que, em média, as melhores soluções são encontradas para $\sigma=0,5$ e $\delta_{\text {ini }}=1,00$, sendo a melhora média próxima de $27 \%$, e a menor melhora média é encontrada quando $\sigma=0,1$ e $\delta_{\text {ini }}=0,10$, sendo um pouco abaixo de 3\%. Fazendo a mesma análise, mas agora para os sete conjuntos (Figura 4.30b), vemos que, como esperado, a melhora média diminui, sendo a maior quando $\sigma=0,5$ e $\delta_{\text {ini }}=1,00$ (aproximadamente $22 \%$ ) e a menor quando $\sigma=0,1$ e $\delta_{\text {ini }}=0,10$ (cerca de $1 \%$ ).

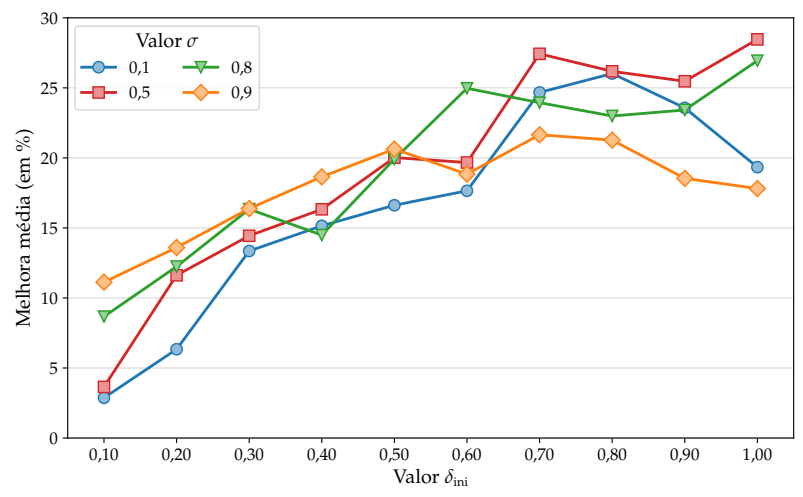

(a) Melhora média para os conjuntos 1 a 5

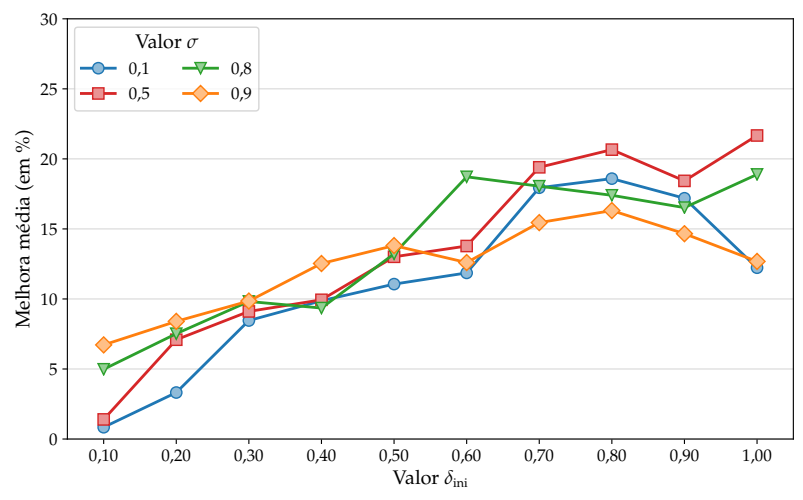

(b) Melhora média para os conjuntos 1 a 7

Figura 4.30: Melhora média da PDA considerando todas as instâncias testadas nos sete conjuntos de problemas testes.

Podemos também fazer uma análise da melhora média para os diferentes valores de $\delta_{\text {ini }}$ (para todos os valores de $\sigma$ ) e vice-versa, como mostra os gráficos da Figura 4.31. Pelo gráfico da Figura 4.31a vemos que a PDA obtém melhores resultados para $\delta_{\text {ini }}=0,70$, quando não levamos em conta os conjuntos 6 e 7. Considerando esses conjuntos, a PDA obtém a maior melhora média para $\delta_{\text {ini }}=$ 
0,80. O pior resultado da PDA, considerando ou não os conjuntos 6 e 7, ocorre quando $\delta_{\text {ini }}=0,10$. Analisando o efeito do valor do $\sigma$ na PDA, para os diferentes valores de $\delta_{\text {ini }}$, o gráfico da Figura $4.31 \mathrm{~b}$ mostra que a PDA consegue melhores resultados quando $\sigma \in\{0,5 ; 0,8\}$. Portanto, pela melhora média, uma boa escolha de $\sigma$ e $\delta_{\text {ini }}$ para a PDA seria $\sigma \in\{0,5 ; 0,8\}$ e $\delta_{\text {ini }} \in\{0,70 ; 0,80\}$.

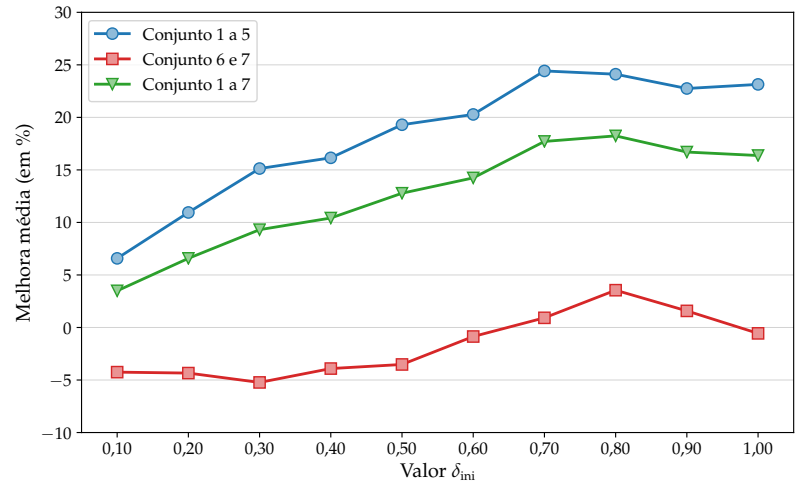

(a) Melhora média por $\delta_{\text {ini }}$

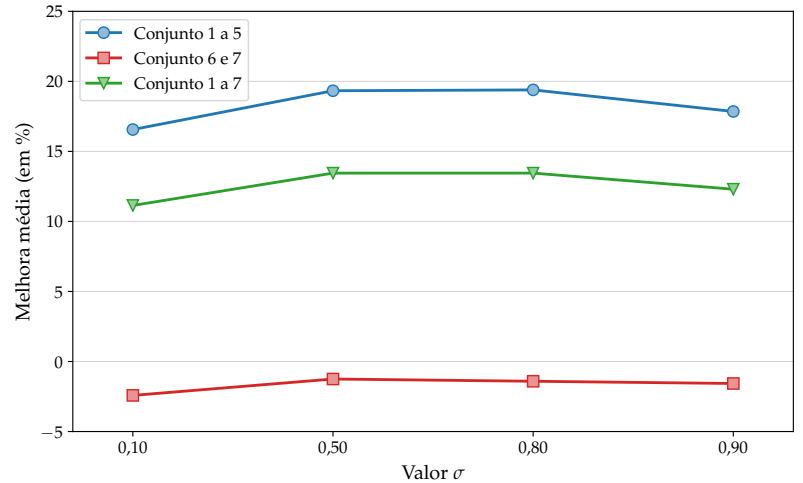

(b) Melhora média por $\sigma$

Figura 4.31: Melhora média geral da PDA. 


\section{Capítulo 5}

\section{Conclusões}

Neste trabalho, estudamos o problema de corte bidimensional multiperíodo com sobras aproveitáveis (PCBMPSA). Baseado no trabalho de [6], apresentamos um modelo de programação linear inteira mista para o PCBMPSA, cujo objetivo é cortar os itens solicitados nas ordens de trabalho de todos os períodos do horizonte de planejamento usando um conjunto de objetos de custo mínimo ou as sobras geradas no corte em algum período e, entre todas as possíveis soluções de custo mínimo, escolher uma que maximize o valor das sobras aproveitáveis do último período. A formulação leva em conta que sobras geradas durante o processo de corte dos itens de um período podem ser utilizadas como novos objetos em períodos futuros, o que pode diminuir o custo dos objetos necessários para atender a ordem de trabalho dos itens de todos os períodos. Como o número de sobras cresce exponencialmente, já que cada objeto pode gerar duas sobras, também consideramos o caso em que as sobras geradas em um período ficam disponíveis por, no máximo, $\xi$ períodos e depois são descartadas (caso não tenham sido utilizadas). Apresentamos três modelos $\left(\mathcal{M}_{p, P}^{1}, \mathcal{M}_{p, P}^{2}\right.$ e $\left.\mathcal{M}_{p, P}^{3}\right)$, que levam em conta a permissão ou não da compra e o eventual corte de objetos de entrada em um determinado período, mesmo que nenhum item no período seja atribuído ao objeto.

Considerando a dificuldade em resolver de forma exata instâncias médias e grandes (comuns na prática) de modelos MIP para o problema PCBMPSA, apresentamos quatro métodos heurísticos, comumente utilizadas para problemas de programação linear inteira multiperíodo, para auxiliar na resolução do PCBMPSA: método guloso, horizonte rolante, relax-and-fix e programação dinâmica aproximada.

$\mathrm{Na}$ estratégia gulosa resolvemos o problema considerando cada período de forma individual e as sobras aproveitáveis ficam disponíveis para serem utilizados no corte dos itens de ordens de trabalho dos períodos seguintes. No horizonte rolante, o horizonte de planejamento é dividido em três partes (ou janelas). Na primeira parte (chamada de janela congelada) as variáveis de decisão, pertencentes aos períodos dessa parte, tem seus valores fixados. A segunda parte (janela de decisão) contém os dados dos períodos que serão resolvidos. A última parte (janela ignorada) possui os dados que ainda não foram considerados, sendo as variáveis dos períodos dessa parte ignoradas. Além da forma tradicional apresentada para o horizonte rolante, com o objetivo de melhorar a qualidade das soluções encontradas pelo método, também permitimos a sobreposição do horizonte de planejamento 
a ser resolvido entre duas janelas de decisão consecutivas.

O terceiro método considerado para resolver o PCBMPSA é o relax-and-fix, onde o problema original é decomposto em vários subproblemas menores e parcialmente relaxados, que são resolvidos em sequência. Em cada subproblema, apenas variáveis de um subconjunto permanecem com a restrição de integralidade, enquanto que as variáveis restantes ou são fixadas ou têm sua restrição de integralidade relaxada. O relax-and-fix é similar ao horizonte rolante. A diferença é que a janela ignorada do horizonte rolante passa a ser uma janela de aproximação e as variáveis dos períodos dessa janela têm sua integralidade relaxada.

A última estratégia considerada é baseada em Programação Dinâmica Aproximada (PDA). A ideia adotada para resolver o PCBMPSA usando a PDA é estimar a proporção de utilização futura das sobras de cada objeto em cada período. Para calcular essa estimativa, o algoritmo resolve uma versão modificada do modelo proposto para o PCBMPSA. Tal modelo considera os dados de cada período de forma individual (como feito no método guloso), mas a função objetivo leva em conta a estimativa de proporção de utilização futura das sobras para determinar quais objetos, em cada período, possuem o melhor custo-benefício. Quando o algoritmo resolve o problema para todos os períodos do horizonte de planejamento, as estimativas de proporção de utilização das sobras são atualizadas e o método é reiniciado, mas agora considerando as novas estimativas.

Para verificar a eficiência de cada método heurístico proposto, realizamos alguns experimentos numéricos. A PDA se mostrou o método mais eficiente ao conseguir melhores resultados na maioria das instâncias testadas. Além disso, o tempo gasto por ela foi bem menor que o tempo necessário em algumas versões do horizonte rolante e relax-and-fix. Pelos experimentos também observarmos que o relax-and-fix, mesmo "usando" as informações futuras relaxadas, não conseguiu melhores resultado que o horizonte rolante, como era esperado, ou seja, a relaxação das variáveis e restrições de alguns períodos não foi o suficiente para que o método se comportasse melhor que o horizonte rolante.

Finalmente, pensando em um contexto mais prático do PCBMPSA, também consideramos o cenário onde não conhecemos à priori a quantidade dos itens solicitados nas ordens de trabalho e o custo dos objetos, mas temos informações das distribuições de probabilidade de ambos. Para esse caso, apresentamos uma abordagem baseada em programação dinâmica aproximada para determinar a melhor estratégia a ser seguida em cada período do horizonte de planejamento. Essa estratégia consiste em estimar os valores das estimativas de proporção de utilização das sobras dos objetos de cada período, utilizando amostras geradas a partir das distribuições de probabilidades da quantidade dos itens e do custo dos objetos. Realizamos alguns experimentos numéricos e comparamos os resultados obtidos pela PDA com os resultados encontrados por um método guloso. Em cenários adequados, os resultados mostraram que a PDA consegue soluções superiores ao método guloso.

O problema aqui estudado apresenta várias oportunidades em diferentes linhas de pesquisas. Para os modelos apresentados para o PCBMPSA, podem ser explorados outros algoritmos exatos visando melhorar as soluções ou os limitantes inferiores obtidos, gastando um tempo computacional aceitável. Para o método relax-and-fix podem ser propostas restrições adicionais de forma que a relaxação das variáveis da janela de aproximação auxilie na qualidade das soluções encontradas pelo método. Outras estratégias heurísticas também podem ser desenvolvidas para resolver o problema. 
Nossa abordagem para solucionar o PCBMPSA, usando a PDA, considera apenas as estimativas da proporção de utilização futura das sobras. Como o método não leva em conta o custo global da solução, soluções de melhor qualidade podem não ser encontradas. Assim, uma nova possibilidade de trabalho futuro é desenvolver outras estratégias para a PDA. Em alguns cenários não-determinísticos a PDA também não conseguiu bons resultados. Acreditamos que outras abordagens podem ser desenvolvidas para que a PDA encontre melhores soluções, mesmo em cenários desfavoráveis para a PDA. 


\section{Apêndice A}

\section{Instâncias determinísticas}

A Tabela A.1 apresenta os dados das instâncias usadas nos experimentos numéricos da versão determinística do nosso problema. Essas instâncias foram geradas seguindo as ideias apresentadas em [3]. Para cada instância, temos o número que a identifica, o número de períodos, a quantidade de tipos distintos de objetos para cada período e suas respectivas dimensões no formato $N(W \times H)[C]$, tal que $N$ refere-se à quantidade de objetos com dimensões $(W \times H)$ e $C$ corresponde ao custo por unidade de área do objeto daquele tipo. Em seguida, temos as informações dos itens: a quantidade de itens em cada período, a quantidade de itens distintos, o número de itens do catálogo e suas respectivas dimensões no formato $N(W \times H)$, tal que $N$ refere-se a quantidade de itens com dimensões $(W \times H)$. Onde $N$ é omitido significa que existe apenas uma unidade do objeto ou item e onde $C$ é omitido significa que o custo por unidade de área do objeto é 1.

Tabela A.1: Dados das instâncias de testes

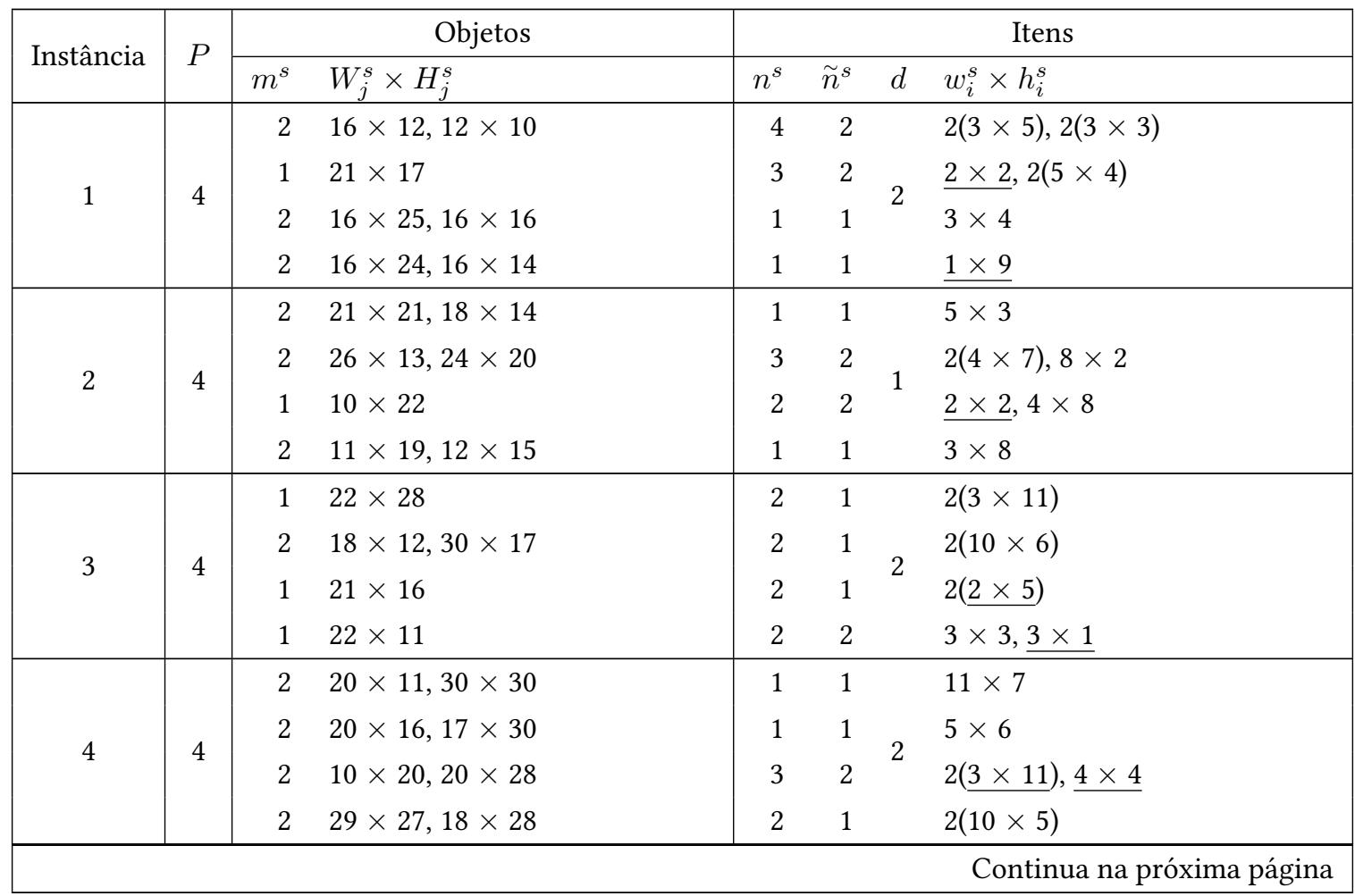


Tabela A.1 - continuação da página anterior

\begin{tabular}{|c|c|c|c|c|c|c|c|}
\hline \multirow{2}{*}{ Instância } & \multirow{2}{*}{$P$} & \multicolumn{2}{|r|}{ Objetos } & \multicolumn{4}{|r|}{ Itens } \\
\hline & & $m^{s}$ & $W_{j}^{s} \times H_{j}^{s}$ & $n^{s}$ & $\tilde{n}^{s}$ & $d$ & $w_{i}^{s} \times h_{i}^{s}$ \\
\hline \multirow{4}{*}{5} & \multirow{4}{*}{4} & 1 & $15 \times 21$ & 3 & 2 & \multirow{4}{*}{1} & $2(5 \times 5), 1 \times 2$ \\
\hline & & 3 & $2(19 \times 18), 23 \times 26$ & 4 & 2 & & $2(6 \times 9), 2(9 \times 11)$ \\
\hline & & 2 & $12 \times 19,23 \times 27$ & 1 & 1 & & $2 \times 6$ \\
\hline & & 2 & $22 \times 27,12 \times 12$ & 2 & 1 & & $2(4 \times 4)$ \\
\hline \multirow{4}{*}{6} & \multirow{4}{*}{4} & 2 & $40 \times 33,29 \times 32$ & 3 & 2 & \multirow{4}{*}{3} & $2(\underline{8 \times 4}), \underline{10 \times 3}$ \\
\hline & & 3 & $40 \times 42,27 \times 46,20 \times 31$ & 3 & 3 & & $8 \times 6,11 \times 4,9 \times 18$ \\
\hline & & 3 & $39 \times 48,2(46 \times 21)$ & 5 & 3 & & $2(19 \times 9), \underline{3 \times 9}, 2(16 \times 17)$ \\
\hline & & 2 & $41 \times 34,34 \times 39$ & 4 & 3 & & $2(6 \times 13), 11 \times 14,3 \times 14$ \\
\hline \multirow{4}{*}{7} & \multirow{4}{*}{4} & 3 & $46 \times 33,39 \times 29,28 \times 44$ & 3 & 2 & \multirow{4}{*}{3} & $2(12 \times 7), 13 \times 11$ \\
\hline & & 2 & $27 \times 48,29 \times 49$ & 2 & 2 & & $\underline{2 \times 18}, 10 \times 19$ \\
\hline & & 2 & $27 \times 32,22 \times 40$ & 3 & 2 & & $\underline{4 \times 13}, 2(6 \times 4)$ \\
\hline & & 2 & $44 \times 39,43 \times 25$ & 3 & 2 & & $2(15 \times 11), \underline{6 \times 2}$ \\
\hline \multirow{4}{*}{8} & \multirow{4}{*}{4} & 2 & $44 \times 38,40 \times 35$ & 3 & 2 & \multirow{4}{*}{1} & $13 \times 6,2(11 \times 15)$ \\
\hline & & 3 & $23 \times 38,26 \times 40,45 \times 31$ & 5 & 3 & & $2(10 \times 14), 13 \times 7,2(18 \times 8)$ \\
\hline & & 4 & $2(24 \times 29), 34 \times 42,47 \times 49$ & 3 & 2 & & $2(17 \times 17), 12 \times 8$ \\
\hline & & 3 & $43 \times 47,34 \times 33,42 \times 43$ & 3 & 2 & & $2(\underline{4 \times 4}), 14 \times 5$ \\
\hline \multirow{4}{*}{9} & \multirow{4}{*}{4} & 4 & $2(25 \times 41), 48 \times 21,43 \times 26$ & 6 & 3 & \multirow{4}{*}{3} & $2(\underline{8 \times 3}), 2(14 \times 17), 2(17 \times 7)$ \\
\hline & & 3 & $28 \times 20,25 \times 30,35 \times 47$ & 5 & 3 & & $2(12 \times 7), \underline{5 \times 4}, 2(\underline{3 \times 14})$ \\
\hline & & 4 & $2(41 \times 22), 49 \times 49,45 \times 25$ & 4 & 3 & & $14 \times 12,2(9 \times 13), 13 \times 16$ \\
\hline & & 3 & $30 \times 37,28 \times 37,41 \times 46$ & 5 & 3 & & $2(5 \times 14), 12 \times 17,2(15 \times 11)$ \\
\hline \multirow{4}{*}{10} & \multirow{4}{*}{4} & 3 & $49 \times 30,47 \times 39,38 \times 29$ & 3 & 2 & \multirow{4}{*}{4} & $2(14 \times 10), 8 \times 12$ \\
\hline & & 3 & $38 \times 29,39 \times 46,39 \times 46$ & 3 & 2 & & $\underline{2 \times 13}, 2(7 \times 12)$ \\
\hline & & 3 & $42 \times 23,30 \times 38,31 \times 26$ & 5 & 3 & & $2(\underline{9 \times 7}), 11 \times 7,2(\underline{3 \times 9})$ \\
\hline & & 2 & $34 \times 40,46 \times 27$ & 2 & 2 & & $\underline{16 \times 6}, 9 \times 10$ \\
\hline \multirow{8}{*}{11} & \multirow{8}{*}{8} & 1 & $12 \times 29$ & 1 & 1 & & $5 \times 9$ \\
\hline & & 2 & $14 \times 23,21 \times 21$ & 4 & 2 & & $2(5 \times 7), 2(2 \times 9)$ \\
\hline & & 2 & $22 \times 15,26 \times 20$ & 2 & 1 & & $2(\underline{5 \times 3})$ \\
\hline & & 2 & $13 \times 25,29 \times 13$ & 3 & 2 & 3 & $2(\underline{6 \times 2}), 12 \times 10$ \\
\hline & & 1 & $30 \times 13$ & 3 & 2 & $J$ & $3 \times 6,2(11 \times 5)$ \\
\hline & & 1 & $17 \times 25$ & 2 & 2 & & $5 \times 7,6 \times 8$ \\
\hline & & 2 & $24 \times 30,30 \times 13$ & 1 & 1 & & $10 \times 8$ \\
\hline & & 1 & $19 \times 26$ & 2 & 1 & & $2(\underline{2 \times 6})$ \\
\hline & & 2 & $19 \times 30,23 \times 22$ & 2 & 2 & & $5 \times 8,5 \times 10$ \\
\hline & & 1 & $30 \times 22$ & 3 & 2 & & $2(7 \times 9), 10 \times 5$ \\
\hline & & 1 & $17 \times 28$ & 3 & 2 & & $2(5 \times 11), 5 \times 4$ \\
\hline 12 & 8 & 1 & $21 \times 10$ & 2 & 2 & 2 & $\underline{5 \times 1}, 5 \times 4$ \\
\hline 14 & & 1 & $20 \times 21$ & 2 & 1 & 2 & $2(\underline{1 \times 5})$ \\
\hline & & 1 & $18 \times 20$ & 2 & 1 & & $2(5 \times 3)$ \\
\hline & & 1 & $28 \times 28$ & 2 & 1 & & $2(7 \times 7)$ \\
\hline & & 3 & $2(27 \times 12), 20 \times 29$ & 4 & 2 & & $2(11 \times 8), 2(7 \times 12)$ \\
\hline
\end{tabular}


Tabela A.1 - continuação da página anterior

\begin{tabular}{|c|c|c|c|c|c|c|c|}
\hline \multirow{2}{*}{ Instância } & \multirow{2}{*}{$P$} & \multicolumn{2}{|r|}{ Objetos } & \multicolumn{4}{|r|}{ Itens } \\
\hline & & $m^{s}$ & $W_{j}^{s} \times H_{j}^{s}$ & $n^{s}$ & $\tilde{n}^{s}$ & $d$ & $w_{i}^{s} \times h_{i}^{s}$ \\
\hline \multirow{8}{*}{13} & \multirow{8}{*}{8} & 1 & $15 \times 24$ & 3 & 2 & \multirow{8}{*}{2} & $1 \times 6,2(3 \times 2)$ \\
\hline & & 2 & $24 \times 24,22 \times 23$ & 1 & 1 & & $10 \times 10$ \\
\hline & & 2 & $27 \times 10,14 \times 16$ & 3 & 2 & & $3(11 \times 3)$ \\
\hline & & 1 & $24 \times 17$ & 2 & 1 & & $2(3 \times 6)$ \\
\hline & & 1 & $29 \times 14$ & 4 & 2 & & $2(12 \times 3), 2(10 \times 4)$ \\
\hline & & 1 & $22 \times 13$ & 3 & 2 & & $5 \times 6,2(8 \times 2)$ \\
\hline & & 1 & $23 \times 17$ & 4 & 2 & & $2(4 \times 3), 2(10 \times 7)$ \\
\hline & & 2 & $14 \times 29,11 \times 25$ & 2 & 1 & & $2(4 \times 5)$ \\
\hline \multirow{8}{*}{14} & \multirow{8}{*}{8} & 2 & $10 \times 17,26 \times 15$ & 3 & 2 & \multirow{8}{*}{2} & $2(\underline{6 \times 2}), 11 \times 2$ \\
\hline & & 1 & $20 \times 28$ & 2 & 1 & & $2(3 \times 8)$ \\
\hline & & 2 & $16 \times 22,15 \times 28$ & 3 & 2 & & $2(\underline{1 \times 3}), 3 \times 8$ \\
\hline & & 2 & $19 \times 17,23 \times 25$ & 2 & 1 & & $2(3 \times 3)$ \\
\hline & & 2 & $13 \times 18,14 \times 21$ & 1 & 1 & & $6 \times 2$ \\
\hline & & 1 & $22 \times 30$ & 3 & 2 & & $6 \times 10,2(9 \times 11)$ \\
\hline & & 2 & $26 \times 13,24 \times 14$ & 3 & 2 & & $4 \times 4,2(6 \times 3)$ \\
\hline & & 1 & $17 \times 24$ & 1 & 1 & & $4 \times 4$ \\
\hline \multirow{8}{*}{15} & \multirow{8}{*}{8} & 1 & $16 \times 24$ & 2 & 1 & \multirow{8}{*}{1} & $2(5 \times 9)$ \\
\hline & & 1 & $17 \times 14$ & 4 & 2 & & $4(\underline{1 \times 2})$ \\
\hline & & 1 & $28 \times 10$ & 2 & 2 & & $5 \times 3,11 \times 4$ \\
\hline & & 2 & $29 \times 16,17 \times 20$ & 2 & 1 & & $2(5 \times 8)$ \\
\hline & & 2 & $23 \times 13,25 \times 25$ & 1 & 1 & & $4 \times 5$ \\
\hline & & 2 & $17 \times 29,21 \times 14$ & 1 & 1 & & $9 \times 3$ \\
\hline & & 1 & $21 \times 22$ & 1 & 1 & & $9 \times 5$ \\
\hline & & 2 & $29 \times 25,23 \times 26$ & 2 & 1 & & $2(10 \times 11)$ \\
\hline \multirow{8}{*}{16} & \multirow{8}{*}{8} & 2 & $24 \times 21,25 \times 44$ & 4 & 2 & \multirow{8}{*}{3} & $2(10 \times 6), 2(\underline{2 \times 8})$ \\
\hline & & 2 & $49 \times 44,41 \times 28$ & 3 & 2 & & $\underline{5 \times 4,2(19 \times 12)}$ \\
\hline & & 2 & $29 \times 45,28 \times 37$ & 4 & 2 & & $2(6 \times 13), 2(9 \times 9)$ \\
\hline & & 3 & $32 \times 47,44 \times 41,44 \times 46$ & 3 & 2 & & $2(13 \times 15), 11 \times 9$ \\
\hline & & 3 & $41 \times 42,27 \times 28,39 \times 34$ & 4 & 3 & & $8 \times 17,15 \times 13,2(9 \times 4)$ \\
\hline & & 2 & $29 \times 41,49 \times 48$ & 2 & 2 & & $20 \times 16,14 \times 18$ \\
\hline & & 2 & $42 \times 24,31 \times 31$ & 5 & 3 & & $2(12 \times 13), 5 \times 10,2(\underline{16 \times 3})$ \\
\hline & & 3 & $37 \times 49,34 \times 42,25 \times 22$ & 3 & 2 & & $2(5 \times 7), 2 \times 11$ \\
\hline \multirow{8}{*}{17} & \multirow{8}{*}{8} & 2 & $47 \times 27,43 \times 25$ & 2 & 2 & \multirow{8}{*}{2} & $17 \times 2,9 \times 5$ \\
\hline & & 3 & $30 \times 36,33 \times 45,26 \times 22$ & 5 & 3 & & $2(6 \times 10), 2(12 \times 11), 8 \times 5$ \\
\hline & & 2 & $29 \times 41,35 \times 36$ & 4 & 3 & & $7 \times 17,2(4 \times 17), 11 \times 9$ \\
\hline & & 3 & $30 \times 47,24 \times 36,50 \times 48$ & 4 & 3 & & $3 \times 5,7 \times 15,2(17 \times 17)$ \\
\hline & & 2 & $44 \times 29,34 \times 22$ & 2 & 2 & & $17 \times 10,3 \times 4$ \\
\hline & & 3 & $46 \times 22,27 \times 43,25 \times 42$ & 3 & 3 & & $3 \times 16,12 \times 17,4 \times 16$ \\
\hline & & 2 & $41 \times 45,39 \times 48$ & 2 & 2 & & $8 \times 17,8 \times 9$ \\
\hline & & 4 & $26 \times 35,2(21 \times 29), 40 \times 44$ & 6 & 3 & & $2(11 \times 14), 2(11 \times 6), 2(3 \times 16)$ \\
\hline & & & & & & & Continua na próxima página \\
\hline
\end{tabular}


Tabela A.1 - continuação da página anterior

\begin{tabular}{|c|c|c|c|c|c|c|c|}
\hline \multirow{2}{*}{ Instância } & \multirow{2}{*}{$P$} & \multicolumn{2}{|r|}{ Objetos } & \multicolumn{4}{|r|}{ Itens } \\
\hline & & $m^{s}$ & $W_{j}^{s} \times H_{j}^{s}$ & $n^{s}$ & $\tilde{n}^{s}$ & $d$ & $w_{i}^{s} \times h_{i}^{s}$ \\
\hline \multirow{8}{*}{18} & \multirow{8}{*}{8} & 5 & $2(49 \times 26), 37 \times 49,2(49 \times 26)$ & 4 & 2 & \multirow{8}{*}{3} & $2(17 \times 19), 2(16 \times 13)$ \\
\hline & & 2 & $21 \times 43,30 \times 44$ & 5 & 3 & & $2(9 \times 17), 2(4 \times 16), 9 \times 9$ \\
\hline & & 3 & $30 \times 31,22 \times 36,48 \times 20$ & 2 & 2 & & $13 \times 14,9 \times 15$ \\
\hline & & 2 & $21 \times 29,21 \times 44$ & 4 & 2 & & $2(6 \times 4), 2(\underline{4 \times 4})$ \\
\hline & & 2 & $48 \times 20,40 \times 39$ & 4 & 3 & & $2(13 \times 5), 13 \times 3,17 \times 14$ \\
\hline & & 2 & $35 \times 28,41 \times 37$ & 2 & 2 & & $10 \times 5,9 \times 8$ \\
\hline & & 2 & $38 \times 48,26 \times 30$ & 3 & 2 & & $3 \times 11,2(\underline{5 \times 3})$ \\
\hline & & 3 & $27 \times 23,45 \times 20,47 \times 21$ & 4 & 3 & & $7 \times 3,2(7 \times 4), 3 \times 10$ \\
\hline \multirow{8}{*}{19} & \multirow{8}{*}{8} & 3 & $20 \times 38,20 \times 32,23 \times 33$ & 3 & 2 & \multirow{8}{*}{2} & $\underline{2 \times 8}, 2(6 \times 9)$ \\
\hline & & 2 & $43 \times 38,36 \times 35$ & 2 & 2 & & $4 \times 6,10 \times 10$ \\
\hline & & 2 & $49 \times 31,39 \times 37$ & 6 & 3 & & $2(8 \times 15), 2(\underline{4 \times 2}), 2(15 \times 4)$ \\
\hline & & 2 & $43 \times 21,49 \times 43$ & 4 & 3 & & $14 \times 9,4 \times 10,2(6 \times 18)$ \\
\hline & & 3 & $48 \times 44,32 \times 34,28 \times 29$ & 3 & 2 & & $2(10 \times 16), 14 \times 3$ \\
\hline & & 2 & $42 \times 23,36 \times 43$ & 4 & 3 & & $3 \times 12,2(6 \times 6), 10 \times 5$ \\
\hline & & 3 & $50 \times 39,46 \times 43,49 \times 27$ & 3 & 2 & & $11 \times 12,2(8 \times 6)$ \\
\hline & & 3 & $39 \times 38,38 \times 40,21 \times 29$ & 3 & 2 & & $4 \times 10,2(7 \times 8)$ \\
\hline \multirow{8}{*}{20} & \multirow{8}{*}{8} & 3 & $28 \times 29,44 \times 46,31 \times 20$ & 3 & 2 & \multirow{8}{*}{3} & $13 \times 5,2(12 \times 14)$ \\
\hline & & 4 & $45 \times 32,2(32 \times 20), 41 \times 35$ & 5 & 3 & & $15 \times 10,2(\underline{4 \times 3}), 2(12 \times 14)$ \\
\hline & & 3 & $39 \times 34,38 \times 32,46 \times 38$ & 5 & 3 & & $3(5 \times 3), 2(10 \times 5)$ \\
\hline & & 2 & $25 \times 27,47 \times 29$ & 5 & 3 & & $2(13 \times 3), 4 \times 9,2(\underline{3 \times 10})$ \\
\hline & & 5 & $2(38 \times 33), 2(40 \times 31), 49 \times 41$ & 5 & 3 & & $5 \times 10,2(16 \times 14), 2(20 \times 12)$ \\
\hline & & 2 & $29 \times 27,30 \times 40$ & 3 & 2 & & $\underline{10 \times 2}, 2(6 \times 4)$ \\
\hline & & 2 & $40 \times 48,35 \times 20$ & 3 & 2 & & $7 \times 18,2(14 \times 20)$ \\
\hline & & 3 & $23 \times 25,48 \times 20,27 \times 28$ & 2 & 2 & & $7 \times 9,19 \times 10$ \\
\hline \multirow{12}{*}{21} & \multirow{12}{*}{12} & 2 & $10 \times 10,16 \times 17$ & 1 & 1 & \multirow{12}{*}{3} & $2 \times 6$ \\
\hline & & 1 & $14 \times 25$ & 3 & 2 & & $4 \times 8,2(2 \times 5)$ \\
\hline & & 2 & $16 \times 23,16 \times 29$ & 3 & 2 & & $3(6 \times 8)$ \\
\hline & & 1 & $27 \times 12$ & 3 & 2 & & $2(\underline{10 \times 1)}, 6 \times 4$ \\
\hline & & 1 & $17 \times 29$ & 3 & 2 & & $3(5 \times 7)$ \\
\hline & & 1 & $13 \times 24$ & 2 & 2 & & $6 \times 4, \underline{2 \times 3}$ \\
\hline & & 2 & $24 \times 29,10 \times 23$ & 1 & 1 & & $10 \times 11$ \\
\hline & & 1 & $18 \times 21$ & 2 & 1 & & $2(3 \times 5)$ \\
\hline & & 2 & $13 \times 27,27 \times 21$ & 2 & 2 & & $8 \times 8,3 \times 5$ \\
\hline & & 2 & $21 \times 18,11 \times 15$ & 4 & 2 & & $2(5 \times 5), 2(4 \times 3)$ \\
\hline & & 1 & $21 \times 12$ & 2 & 1 & & $2(\underline{5 \times 2})$ \\
\hline & & 1 & $12 \times 21$ & 2 & 2 & & $4 \times 9,2 \times 5$ \\
\hline
\end{tabular}


Tabela A.1 - continuação da página anterior

\begin{tabular}{|c|c|c|c|c|c|c|c|}
\hline \multirow{2}{*}{ Instância } & \multirow{2}{*}{$P$} & \multicolumn{2}{|r|}{ Objetos } & \multicolumn{4}{|r|}{ Itens } \\
\hline & & $m^{s}$ & $W_{j}^{s} \times H_{j}^{s}$ & $n^{s}$ & $\tilde{n}^{s}$ & $d$ & $w_{i}^{s} \times h_{i}^{s}$ \\
\hline \multirow{12}{*}{22} & \multirow{12}{*}{12} & 2 & $13 \times 19,24 \times 29$ & 2 & 1 & \multirow{12}{*}{2} & $2(8 \times 7)$ \\
\hline & & 1 & $18 \times 16$ & 1 & 1 & & $5 \times 7$ \\
\hline & & 1 & $29 \times 27$ & 2 & 1 & & $2(6 \times 5)$ \\
\hline & & 2 & $18 \times 14,17 \times 12$ & 2 & 2 & & $8 \times 5,8 \times 2$ \\
\hline & & 1 & $16 \times 23$ & 4 & 2 & & $2(\underline{1 \times 10)}, 2(7 \times 2)$ \\
\hline & & 2 & $20 \times 20,23 \times 16$ & 3 & 2 & & $3 \times 5,2(3 \times 4)$ \\
\hline & & 2 & $20 \times 26,15 \times 23$ & 4 & 2 & & $2(5 \times 10), 2(4 \times 2)$ \\
\hline & & 1 & $28 \times 12$ & 3 & 2 & & $2(8 \times 4), 12 \times 5$ \\
\hline & & 2 & $24 \times 15,10 \times 26$ & 2 & 1 & & $2(2 \times 10)$ \\
\hline & & 1 & $23 \times 27$ & 2 & 2 & & $10 \times 8,9 \times 3$ \\
\hline & & 1 & $12 \times 10$ & 2 & 1 & & $2(\underline{2 \times 1})$ \\
\hline & & 1 & $15 \times 13$ & 4 & 2 & & $2(5 \times 1), 2(3 \times 3)$ \\
\hline \multirow{12}{*}{23} & \multirow{12}{*}{12} & 2 & $27 \times 11,18 \times 28$ & 2 & 1 & \multirow{12}{*}{2} & $2(\underline{2 \times 4})$ \\
\hline & & 2 & $20 \times 23,16 \times 29$ & 1 & 1 & & $3 \times 10$ \\
\hline & & 1 & $30 \times 21$ & 1 & 1 & & $10 \times 6$ \\
\hline & & 2 & $28 \times 11,21 \times 16$ & 1 & 1 & & $4 \times 2$ \\
\hline & & 2 & $28 \times 19,26 \times 26$ & 2 & 2 & & $4 \times 2,7 \times 2$ \\
\hline & & 2 & $15 \times 19,11 \times 12$ & 2 & 1 & & $2(2 \times 8)$ \\
\hline & & 2 & $21 \times 13,24 \times 30$ & 4 & 2 & & $2(7 \times 2), 2(2 \times 7)$ \\
\hline & & 2 & $15 \times 10,30 \times 11$ & 3 & 2 & & $2(8 \times 2), 9 \times 2$ \\
\hline & & 1 & $10 \times 19$ & 1 & 1 & & $2 \times 7$ \\
\hline & & 1 & $23 \times 12$ & 3 & 2 & & $2(10 \times 3), 3 \times 1$ \\
\hline & & 1 & $16 \times 24$ & 3 & 2 & & $7 \times 5,2(4 \times 9)$ \\
\hline & & 2 & $13 \times 17,29 \times 27$ & 3 & 2 & & $12 \times 10,2(5 \times 6)$ \\
\hline \multirow{12}{*}{24} & \multirow{12}{*}{12} & 2 & $12 \times 12,11 \times 15$ & 4 & 2 & \multirow{12}{*}{3} & $2(4 \times 6), 2(\underline{1 \times 6})$ \\
\hline & & 1 & $22 \times 30$ & 2 & 1 & & $2(4 \times 3)$ \\
\hline & & 1 & $21 \times 19$ & 2 & 1 & & $2(7 \times 1)$ \\
\hline & & 1 & $11 \times 30$ & 2 & 1 & & $2(2 \times 7)$ \\
\hline & & 1 & $10 \times 12$ & 3 & 2 & & $\underline{2 \times 2}, 2(\underline{3 \times 1})$ \\
\hline & & 2 & $23 \times 27,10 \times 17$ & 1 & 1 & & $3 \times 7$ \\
\hline & & 2 & $26 \times 29,14 \times 28$ & 1 & 1 & & $4 \times 8$ \\
\hline & & 2 & $28 \times 29,25 \times 28$ & 2 & 1 & & $2(12 \times 12)$ \\
\hline & & 1 & $19 \times 24$ & 1 & 1 & & $3 \times 6$ \\
\hline & & 2 & $22 \times 17,12 \times 18$ & 3 & 2 & & $2(5 \times 6), 9 \times 5$ \\
\hline & & 2 & $23 \times 13,12 \times 21$ & 1 & 1 & & $5 \times 2$ \\
\hline & & 2 & $18 \times 19,15 \times 25$ & 3 & 2 & & $2(2 \times 3), 4 \times 2$ \\
\hline
\end{tabular}


Tabela A.1 - continuação da página anterior

\begin{tabular}{|c|c|c|c|c|c|c|c|}
\hline \multirow{2}{*}{ Instância } & \multirow{2}{*}{$P$} & \multicolumn{2}{|r|}{ Objetos } & \multicolumn{4}{|r|}{ Itens } \\
\hline & & $m^{s}$ & $W_{j}^{s} \times H_{j}^{s}$ & $n^{s}$ & $\tilde{n}^{s}$ & $d$ & $w_{i}^{s} \times h_{i}^{s}$ \\
\hline \multirow{12}{*}{25} & \multirow{12}{*}{12} & 1 & $26 \times 29$ & 3 & 2 & \multirow{12}{*}{2} & $2(\underline{3 \times 3}), 6 \times 3$ \\
\hline & & 1 & $17 \times 25$ & 4 & 2 & & $2(7 \times 3), 2(2 \times 10)$ \\
\hline & & 1 & $15 \times 10$ & 2 & 1 & & $2(\underline{3 \times 3})$ \\
\hline & & 2 & $10 \times 20,30 \times 19$ & 3 & 2 & & $2(12 \times 3), 2 \times 8$ \\
\hline & & 2 & $17 \times 20,25 \times 30$ & 3 & 2 & & $2(9 \times 5), 5 \times 9$ \\
\hline & & 1 & $25 \times 12$ & 2 & 1 & & $2(7 \times 4)$ \\
\hline & & 1 & $11 \times 23$ & 2 & 2 & & $5 \times 10,4 \times 7$ \\
\hline & & 1 & $20 \times 23$ & 2 & 1 & & $2(3 \times 3)$ \\
\hline & & 1 & $21 \times 18$ & 1 & 1 & & $8 \times 4$ \\
\hline & & 1 & $20 \times 14$ & 3 & 2 & & $2(\underline{1 \times 4}), 7 \times 4$ \\
\hline & & 2 & $24 \times 28,10 \times 10$ & 1 & 1 & & $4 \times 9$ \\
\hline & & 1 & $23 \times 20$ & 3 & 2 & & $2(4 \times 7), 6 \times 3$ \\
\hline \multirow{12}{*}{26} & \multirow{12}{*}{12} & 3 & $46 \times 29,48 \times 30,33 \times 35$ & 5 & 3 & \multirow{12}{*}{3} & $4(4 \times 10), 20 \times 7$ \\
\hline & & 2 & $41 \times 43,45 \times 30$ & 3 & 2 & & $2(5 \times 7), 11 \times 3$ \\
\hline & & 3 & $48 \times 21,46 \times 26,47 \times 23$ & 4 & 3 & & $15 \times 4,2(10 \times 7), 20 \times 6$ \\
\hline & & 3 & $21 \times 29,31 \times 42,21 \times 39$ & 3 & 2 & & $2(2 \times 10), 11 \times 17$ \\
\hline & & 4 & $26 \times 42,50 \times 32,2(38 \times 20)$ & 5 & 3 & & $8 \times 14,2(13 \times 17), 2(13 \times 8)$ \\
\hline & & 2 & $43 \times 49,44 \times 34$ & 5 & 3 & & $2(4 \times 11), 2(11 \times 19), 18 \times 19$ \\
\hline & & 3 & $32 \times 34,40 \times 48,35 \times 37$ & 4 & 3 & & $2(12 \times 13), 11 \times 18,16 \times 18$ \\
\hline & & 3 & $32 \times 35,48 \times 29,46 \times 50$ & 4 & 3 & & $19 \times 19,2(6 \times 15), 12 \times 9$ \\
\hline & & 2 & $24 \times 31,25 \times 49$ & 4 & 2 & & $2(4 \times 7), 2(2 \times 15)$ \\
\hline & & 2 & $33 \times 27,48 \times 46$ & 4 & 3 & & $2(3 \times 11), 14 \times 13,7 \times 9$ \\
\hline & & 2 & $20 \times 25,21 \times 39$ & 6 & 3 & & $2(3 \times 16), 2(\underline{8 \times 4}), 2(\underline{9 \times 2})$ \\
\hline & & 3 & $37 \times 41,32 \times 34,35 \times 34$ & 3 & 2 & & $2(14 \times 7), \underline{2 \times 7}$ \\
\hline \multirow{12}{*}{27} & \multirow{12}{*}{12} & 2 & $31 \times 43,30 \times 41$ & 5 & 3 & \multirow{12}{*}{2} & $2 \times 13,2(4 \times 16), 2(4 \times 12)$ \\
\hline & & 3 & $24 \times 35,36 \times 34,41 \times 47$ & 4 & 3 & & $5 \times 5,11 \times 13,2(6 \times 10)$ \\
\hline & & 2 & $30 \times 45,28 \times 50$ & 3 & 2 & & $\underline{2 \times 3}, 2(8 \times 8)$ \\
\hline & & 3 & $48 \times 23,48 \times 32,38 \times 29$ & 4 & 3 & & $\underline{10 \times 2,2(16 \times 6), 15 \times 3}$ \\
\hline & & 2 & $48 \times 38,32 \times 40$ & 3 & 2 & & $2(13 \times 8), 11 \times 4$ \\
\hline & & 3 & $20 \times 37,23 \times 21,32 \times 33$ & 4 & 3 & & $2(5 \times 3), 5 \times 10,13 \times 11$ \\
\hline & & 2 & $21 \times 46,36 \times 39$ & 3 & 2 & & $3 \times 16,2(6 \times 12)$ \\
\hline & & 3 & $38 \times 33,30 \times 22,34 \times 32$ & 3 & 2 & & $2(7 \times 6), 14 \times 14$ \\
\hline & & 4 & $2(25 \times 25), 20 \times 49,45 \times 21$ & 4 & 3 & & $12 \times 18,2(7 \times 16), 5 \times 5$ \\
\hline & & 3 & $27 \times 23,39 \times 29,38 \times 20$ & 4 & 3 & & $10 \times 11,7 \times 8,2(7 \times 7)$ \\
\hline & & 3 & $42 \times 42,37 \times 34,21 \times 23$ & 2 & 2 & & $14 \times 7,3 \times 14$ \\
\hline & & 2 & $49 \times 43,32 \times 25$ & 2 & 2 & & $11 \times 15,9 \times 11$ \\
\hline
\end{tabular}


Tabela A.1 - continuação da página anterior

\begin{tabular}{|c|c|c|c|c|c|c|c|}
\hline \multirow{2}{*}{ Instância } & \multirow{2}{*}{$P$} & \multicolumn{2}{|r|}{ Objetos } & \multicolumn{4}{|r|}{ Itens } \\
\hline & & $m^{s}$ & $W_{j}^{s} \times H_{j}^{s}$ & $n^{s}$ & $\tilde{n}^{s}$ & $d$ & $w_{i}^{s} \times h_{i}^{s}$ \\
\hline \multirow{12}{*}{28} & \multirow{12}{*}{12} & 2 & $45 \times 30,32 \times 36$ & 4 & 3 & \multirow{12}{*}{2} & $13 \times 10,2(9 \times 14), 16 \times 12$ \\
\hline & & 2 & $42 \times 46,21 \times 34$ & 4 & 3 & & $3 \times 16,7 \times 15,2(4 \times 6)$ \\
\hline & & 4 & $43 \times 41,40 \times 24,2(26 \times 22)$ & 4 & 2 & & $2(7 \times 7), 2(16 \times 13)$ \\
\hline & & 4 & $2(20 \times 41), 48 \times 46,40 \times 32$ & 3 & 3 & & $11 \times 18,18 \times 13,17 \times 16$ \\
\hline & & 3 & $32 \times 45,45 \times 35,49 \times 47$ & 4 & 3 & & $9 \times 4,20 \times 11,2(19 \times 16)$ \\
\hline & & 2 & $37 \times 30,50 \times 47$ & 4 & 3 & & $2(13 \times 8), 10 \times 16,18 \times 10$ \\
\hline & & 2 & $30 \times 36,36 \times 28$ & 4 & 3 & & $11 \times 3,9 \times 7,2(\underline{2 \times 3})$ \\
\hline & & 3 & $42 \times 20,25 \times 40,45 \times 28$ & 5 & 3 & & $7 \times 9,2(5 \times 8), 2(6 \times 5)$ \\
\hline & & 2 & $47 \times 30,34 \times 26$ & 5 & 3 & & $8 \times 11,2(15 \times 8), 2(\underline{9 \times 2})$ \\
\hline & & 3 & $49 \times 33,21 \times 43,41 \times 25$ & 2 & 2 & & $4 \times 5,16 \times 14$ \\
\hline & & 3 & $20 \times 50,40 \times 30,33 \times 44$ & 4 & 3 & & $9 \times 16,2(8 \times 19), 12 \times 7$ \\
\hline & & 2 & $24 \times 38,34 \times 47$ & 3 & 2 & & $13 \times 19,2(4 \times 3)$ \\
\hline \multirow{12}{*}{29} & \multirow{12}{*}{12} & 2 & $48 \times 31,35 \times 31$ & 3 & 3 & \multirow{12}{*}{3} & $5 \times 10,14 \times 3,10 \times 11$ \\
\hline & & 3 & $24 \times 46,47 \times 20,49 \times 31$ & 3 & 2 & & $8 \times 6,2(14 \times 8)$ \\
\hline & & 2 & $38 \times 43,33 \times 50$ & 3 & 2 & & $2(13 \times 17), 7 \times 7$ \\
\hline & & 3 & $30 \times 46,23 \times 21,40 \times 27$ & 4 & 3 & & $2(6 \times 11), 14 \times 6,9 \times 13$ \\
\hline & & 4 & $45 \times 43,23 \times 37,2(23 \times 22)$ & 4 & 3 & & $16 \times 18,11 \times 7,2(6 \times 7)$ \\
\hline & & 3 & $37 \times 20,35 \times 31,34 \times 40$ & 4 & 3 & & $14 \times 14, \underline{11 \times 2}, 2(\underline{8 \times 4})$ \\
\hline & & 3 & $27 \times 47,25 \times 47,42 \times 28$ & 3 & 2 & & $2(14 \times 11), 13 \times 4$ \\
\hline & & 3 & $32 \times 23,24 \times 42,36 \times 44$ & 2 & 2 & & $8 \times 5,15 \times 16$ \\
\hline & & 3 & $25 \times 37,38 \times 30,33 \times 43$ & 3 & 2 & & $2(6 \times 9), 2 \times 7$ \\
\hline & & 3 & $25 \times 34,26 \times 35,38 \times 31$ & 6 & 3 & & $2(11 \times 3), 2(15 \times 3), 2(14 \times 14)$ \\
\hline & & 2 & $21 \times 41,47 \times 29$ & 3 & 2 & & $2(16 \times 5), 3 \times 10$ \\
\hline & & 2 & $30 \times 48,47 \times 45$ & 6 & 3 & & $2(5 \times 12), 2(3 \times 14), 2(8 \times 19)$ \\
\hline \multirow{12}{*}{30} & \multirow{12}{*}{12} & 3 & $48 \times 21,41 \times 41,20 \times 39$ & 5 & 3 & \multirow{12}{*}{2} & $8 \times 17,2(3 \times 11), 2(8 \times 15)$ \\
\hline & & 2 & $37 \times 28,38 \times 30$ & 4 & 3 & & $9 \times 9,15 \times 6,2(14 \times 6)$ \\
\hline & & 2 & $38 \times 32,27 \times 38$ & 4 & 3 & & $2(12 \times 10), 14 \times 12,2 \times 14$ \\
\hline & & 3 & $23 \times 27,37 \times 42,35 \times 34$ & 5 & 3 & & $4 \times 5,2(13 \times 6), 2(5 \times 5)$ \\
\hline & & 3 & $29 \times 37,24 \times 27,43 \times 26$ & 3 & 3 & & $7 \times 8, \underline{9 \times 2,10 \times 13}$ \\
\hline & & 2 & $42 \times 34,22 \times 37$ & 5 & 3 & & $2(16 \times 10), 5 \times 7,2(14 \times 8)$ \\
\hline & & 2 & $31 \times 34,45 \times 22$ & 2 & 2 & & $7 \times 11,17 \times 4$ \\
\hline & & 2 & $40 \times 50,33 \times 30$ & 5 & 3 & & $4 \times 10,2(5 \times 11), 2(5 \times 8)$ \\
\hline & & 2 & $35 \times 23,40 \times 21$ & 4 & 2 & & $2(9 \times 10), 2(\underline{2 \times 4})$ \\
\hline & & 3 & $33 \times 43,44 \times 48,32 \times 41$ & 4 & 3 & & $10 \times 16,2(3 \times 4), 17 \times 13$ \\
\hline & & 2 & $35 \times 21,28 \times 36$ & 4 & 3 & & $2(10 \times 5), 14 \times 8,3 \times 4$ \\
\hline & & 3 & $26 \times 41,20 \times 39,29 \times 29$ & 2 & 2 & & $7 \times 7,3 \times 5$ \\
\hline
\end{tabular}




\section{Referências Bibliográficas}

[1] Absi, N. \& Kedad-Sidhoum, S. (2007). MIP-based heuristics for multi-item capacitated lot-sizing problem with setup times and shortage costs. RAIRO-Operations Research, 41(2), 171-192.

[2] Abuabara, A. \& Morabito, R. (2009). Cutting optimization of structural tubes to build agricultural light aircrafts. Annals of Operations Research, 169(1), 149-165.

[3] Andrade, R. (2012). Problemas de corte com sobras aproveitáveis e eliminação de simetrias. Tese de doutorado, Universidade de São Paulo.

[4] Andrade, R., Birgin, E., \& Morabito, R. (2014a). Two-stage two-dimensional guillotine cutting stock problems with usable leftover. International Transactions in Operational Research, 23(1-2), $121-145$.

[5] Andrade, R. \& Birgin, E. G. (2013). Symmetry-breaking constraints for packing identical rectangles within polyhedra. Optimization Letters, 7(2), 375-405.

[6] Andrade, R., Birgin, E. G., Morabito, R., \& Ronconi, D. P. (2014b). MIP models for two-dimensional non-guillotine cutting problems with usable leftovers. Journal of the Operational Research Society, 65(11), 1649-1663.

[7] Armas, J., Miranda, G., \& León, C. (2011). A multi-objective approach for the 2d guillotine cutting stock problem. In J. Cabestany, I. Rojas, \& G. Joya (Eds.), Advances in Computational Intelligence, volume 6692 of Lecture Notes in Computer Science (pp. 292-299). Springer Berlin Heidelberg.

[8] Beasley, J. E. (1985). An exact two-dimensional non-guillotine cutting tree search procedure. Operations Research, 33(1), 49-64.

[9] Bertsekas, D. P. (2005). Dynamic programming and optimal control, volume 1. Athena Scientific Belmont, MA, 3rd edition.

[10] Birgin, E. G., Lobato, R. D., \& Morabito, R. (2010). An effective recursive partitioning approach for the packing of identical rectangles in a rectangle. Journal of the Operational Research Society, 61(2), 306-320.

[11] Birgin, E. G., Lobato, R. D., \& Morabito, R. (2012). Generating unconstrained two-dimensional non-guillotine cutting patterns by a recursive partitioning algorithm. Fournal of the Operational Research Society, 63(2), 183-200. 
[12] Blum, C. \& Schmid, V. (2013). Solving the 2d bin packing problem by means of a hybrid evolutionary algorithm. Procedia Computer Science, 18, 899 - 908.

[13] Bosman, P. A. N. \& La Poutré, H. (2008). Online Transportation and Logistics Using Computationally Intelligent Anticipation, (pp. 185-208). Springer Berlin Heidelberg: Berlin, Heidelberg.

[14] Brown, A. R. (1971). Optimum Packing and Depletion: The Computer in Space - And ResourceUsage Problems. Computer monographs, 14. MacDonald.

[15] Cherri, A. C., Arenales, M. N., \& Yanasse, H. H. (2009). The one-dimensional cutting stock problem with usable leftover - a heuristic approach. European fournal of Operational Research, 196, 897-908.

[16] Cherri, A. C., Arenales, M. N., \& Yanasse, H. H. (2013). The usable leftover one-dimensional cutting stock problem - a priority-in-use heuristic. International Transactions in Operational Research, 20(2), 189-199.

[17] Cherri, A. C., Arenales, M. N., Yanasse, H. H., Poldi, K. C., \& Vianna, A. C. G. (2014). The onedimensional cutting stock problem with usable leftovers-a survey. European fournal of Operational Research, 236(2), 395-402.

[18] Chu, C. \& Antonio, J. (1999). Approximation algorithms to solve real-life multicriteria cutting stock problems. Operations Research, 47(4), 495-508.

[19] Cintra, G., Miyazawa, F., Wakabayashi, Y., \& Xavier, E. (2007). A note on the approximability of cutting stock problems. European fournal of Operational Research, 183(3), 1328 - 1332.

[20] Cui, Y. \& Yang, Y. (2010). A heuristic for the one-dimensional cutting stock problem with usable leftover. European fournal of Operational Research, 204(2), 245-250.

[21] Dempe, S. (2002). Foundations of Bilevel Programming. Nonconvex Optimization and Its Applications. Springer.

[22] Dyckhoff, H. (1981). A new linear programming approach to the cutting stock problem. Operations Research, 29(6), 1092-1104.

[23] Dyckhoff, H. (1990). A typology of cutting and packing problems. European fournal of Operational Research, 44(2), 145 - 159. Cutting and Packing.

[24] Garey, M, R. \& Johnson, D, S. (1990). Computers and Intractability: A Guide to the Theory of NP-Completeness. New York, NY, USA: W. H. Freeman \& Co.

[25] Gilmore, P. C. \& Gomory, R. E. (1961). A linear programming approach to the cutting-stock problem. Operations Research, 9(6), 849-859.

[26] Gilmore, P. C. \& Gomory, R. E. (1965). Multistage cutting stock problems of two and more dimensions. Operations Research, 13(1), 94-120. 
[27] Gradišar, M., Jesenko, J., \& Resinovič, G. (1997). Optimization of roll cutting in clothing industry. Computers and Operations Research, 24(10), 945-953.

[28] Gradišar, M., Kljajić, M., Resinovič, G., \& Jesenko, J. (1999a). A sequential heuristic procedure for one-dimensional cutting. European fournal of Operational Research, 114(3), 557 - 568.

[29] Gradišar, M., Resinovič, G., \& Kljajić, M. (1999b). A hybrid approach for optimization of onedimensional cutting. European Journal of Operational Research, 119(3), 719 - 728.

[30] Gradišar, M. \& Trkman, P. (2005). A combined approach to the solution to the general onedimensional cutting stock problem. Computers and Operations Research, 32(7), 1793-1807.

[31] Gramani, M. C. N. \& França, P. M. (2006). The combined cutting stock and lot-sizing problem in industrial processes. European fournal of Operational Research, 174(1), 509-521.

[32] Harjunkoski, I., Pörn, R., Westerlund, T., \& Skrifvars, H. (1997). Different strategies for solving bilinear integer non-linear programming problems with convex transformations. Computers and Chemical Engineering, 21, Supplement(0), 487-492.

[33] Marques, F. d. P. \& Arenales, M. N. (2002). O Problema da mochila compartimentada e aplicações. Pesquisa Operacional, 22, 285 - 304.

[34] Miettinen, K. (1999). Nonlinear Multiobjective Optimization. International Series in Operations Research \& Management Science. Springer US.

[35] Miranda, G., de Armas, J., Segura, C., \& Leon, C. (2010). Hyperheuristic codification for the multi-objective $2 \mathrm{~d}$ guillotine strip packing problem. In Evolutionary Computation (CEC), 2010 IEEE Congress on (pp. 1-8).

[36] Morabito, R. \& Arenales, M. (2000). Optimizing the cutting of stock plates in a furniture company. International fournal of Production Research, 38(12), 2725-2742.

[37] Morabito, R. \& Morales, S. (1998). A simple and effective recursive procedure for the manufacturer's pallet loading problem. Journal of the Operational Research Society, 49(8), 819-828.

[38] Pisinger, D. \& Sigurd, M. (2007). Using decomposition techniques and constraint programming for solving the two-dimensional bin-packing problem. INFORMS fournal on Computing, 19(1), $36-51$.

[39] Pochet, Y. \& Wolsey, L. (2006). Production Planning by Mixed Integer Programming. Springer Series in Operations Research and Financial Engineering. Springer New York.

[40] Poldi, K. C. (2007). O problema de corte de estoque multiperíodo. Tese de doutorado, Universidade de São Paulo.

[41] Poldi, K. C. \& Arenales, M. N. (2010). O problema de corte de estoque unidimensional multiperíodo. Pesquisa Operacional, 30, 153-174. 
[42] Poltroniere, S. C., Poldi, K. C., Toledo, F. M. B., \& Arenales, M. N. (2008). A coupling cutting stock-lot sizing problem in the paper industry. Annals of Operations Research, 157(1), 91-104.

[43] Powell, W. (2008). Approximate dynamic programming: Lessons from the field. In Simulation Conference, 2008. WSC 2008. Winter (pp. 205-214).

[44] Powell, W. B. (2007). Approximate Dynamic Programming: Solving the curses of dimensionality, volume 703. John Wiley \& Sons.

[45] Reinertsen, H. \& Vossen, T. W. (2010). The one-dimensional cutting stock problem with due dates. European fournal of Operational Research, 201(3), 701-711.

[46] Roodman, G. M. (1986). Near-optimal solutions to one-dimensional cutting stock problems. Computers \& Operations Research, 13(6), 713-719.

[47] Sardiñas, R. Q., Santana, M. R., \& Brindis, E. A. (2006). Genetic algorithm-based multi-objective optimization of cutting parameters in turning processes. Engineering Applications of Artificial Intelligence, 19(2), 127-133.

[48] Scheithauer, G. (1991). A note on handling residual lengths. Optimization, 22(3), 461-466.

[49] Sen, S. \& Higle, J. L. (1999). An introductory tutorial on stochastic linear programming models. Interfaces, 29(2), 33-61.

[50] Silva, E., Alvelos, F., \& de Carvalho, J. V. (2010). An integer programming model for two- and three-stage two-dimensional cutting stock problems. European fournal of Operational Research, 205(3), 699-708.

[51] Silva, E., Alvelos, F., \& de Carvalho, J. V. (2013). Integrating two-dimensional cutting stock and lot-sizing problems. Journal of the Operational Research Society, 65(1), 108-123.

[52] Tiwari, S. \& Chakraborti, N. (2006). Multi-objective optimization of a two-dimensional cutting problem using genetic algorithms. fournal of Materials Processing Technology, 173(3), 384-393.

[53] Trkman, P. \& Gradišar, M. (2007). One-dimensional cutting stock optimization in consecutive time periods. European fournal of Operational Research, 179(2), 291-301.

[54] Wolsey, L. (1998). Integer Programming. Wiley Series in Discrete Mathematics and Optimization. Wiley.

[55] Wäscher, G., Haußner, H., \& Schumann, H. (2007). An improved typology of cutting and packing problems. European fournal of Operational Research, 183(3), 1109 - 1130.

[56] Yanasse, H. H. \& Morabito, R. (2006). Linear models for 1-group two-dimensional guillotine cutting problems. International fournal of Production Research, 44(17), 3471-3491. 